Céline Morin

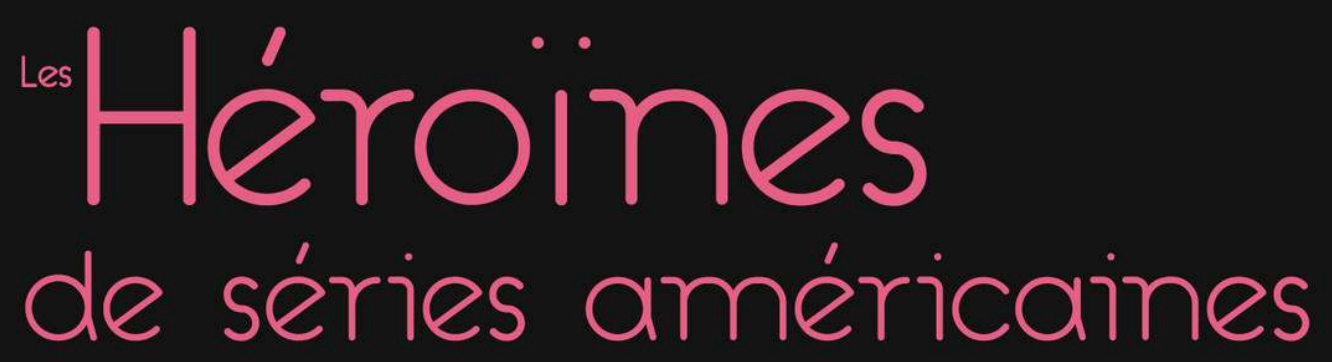

de Ma Sorcière Bien-Aimée à The Good Wife

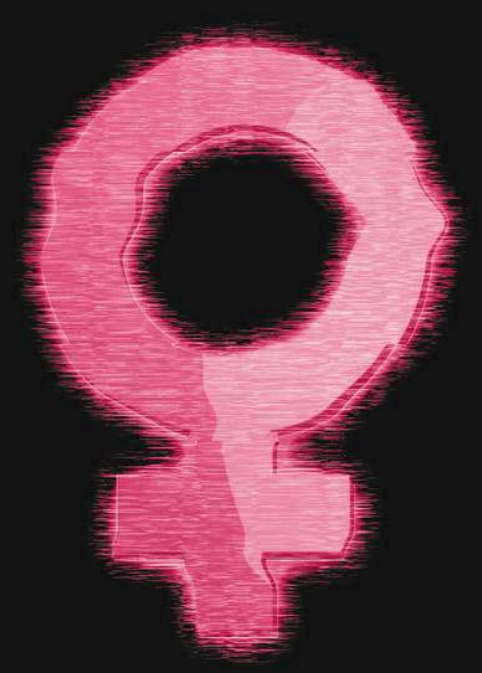




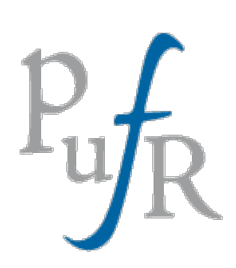

Presses universitaires François-Rabelais

\section{Les Héroïnes de séries américaines}

De Ma Sorcière Bien-Aimée à The Good Wife

\section{Céline Morin}

DOI : 10.4000/books.pufr.9023

Éditeur: Presses universitaires François-Rabelais

Lieu d'édition : Tours

Année d'édition : 2017

Date de mise en ligne : 17 octobre 2018

Collection : Sérial

EAN électronique : 9782869066724

\section{OpenEdition}

\section{Books}

https://books.openedition.org

\section{Édition imprimée}

EAN (Édition imprimée) : 9782869064911

Nombre de pages : 288

\section{Référence électronique}

MORIN, Céline. Les Héroïnes de séries américaines : De Ma Sorcière Bien-Aimée à The Good Wife. Nouvelle édition [en ligne]. Tours : Presses universitaires François-Rabelais, 2017 (généré le 28 janvier 2022). Disponible sur Internet : <http://books.openedition.org/pufr/9023>. ISBN : 9782869066724 . DOI : https://doi.org/10.4000/books.pufr.9023. 


\section{RÉSUMÉS}

Piégée dans son foyer et dans la famille nucléaire classique, Samantha la sorcière était-elle totalement incapable d'émancipation? Est-il possible pour les héroïnes de Sex and the City de réconcilier féminité et féminisme? Les déceptions amoureuses d'Ally McBeal traduisent-elles le dernier sursaut d'un modèle romantique à bout de souffle? Dans The Good Wife, Alicia Florrick, l'épouse trompée redevenue avocate, peut-elle redéployer des compétences juridiques dans sa vie amoureuse?

La fiction américaine a ceci d'extraordinaire qu'elle met en scène tout à la fois les idéologies et fantasmes de l'amour, les conflits entre hommes et femmes dans les espaces privés et publics (du monde du travail au couple et à la famille), les émotions et les intimités vécues des femmes.

Ce livre noue un dialogue avec les théories féministes sans mettre de côté une sociologie de la conjugalité et de l'individualisme. Voici l'analyse la plus approfondie à ce jour des politiques amoureuses et féministes dans la fiction télévisuelle contemporaine.

\section{CÉLINE MORIN}

Spécialiste des séries télévisées, Céline Morin est maître de conférences en sciences de l'information et de la communication à l'université Paris Nanterre. 
Cet ouvrage est diffusé en accès ouvert dans le cadre du projet OpenEdition Books Select.

Ce programme de financement participatif, coordonné par OpenEdition en partenariat avec Knowledge Unlatched et le consortium Couperin, permet aux bibliothèques de contribuer à la libération de contenus provenant d'éditeurs majeurs dans le domaine des sciences humaines et sociales.

La liste des bibliothèques ayant contribué financièrement à la libération de cet ouvrage se trouve ici :

https://www.openedition.org/22515.

This book is published open access as part of the OpenEdition Books Select project.

This crowdfunding program is coordinated by OpenEdition in partnership with Knowledge Unlatched and the French library consortium Couperin. Thanks to the initiative, libraries can contribute to unlatch content from key publishers in the Humanities and Social Sciences.

Discover all the libraries that helped to make this book available open access: https://www.openedition.org/22515?lang=en.

\section{IAI Sopenedition coupernoren}




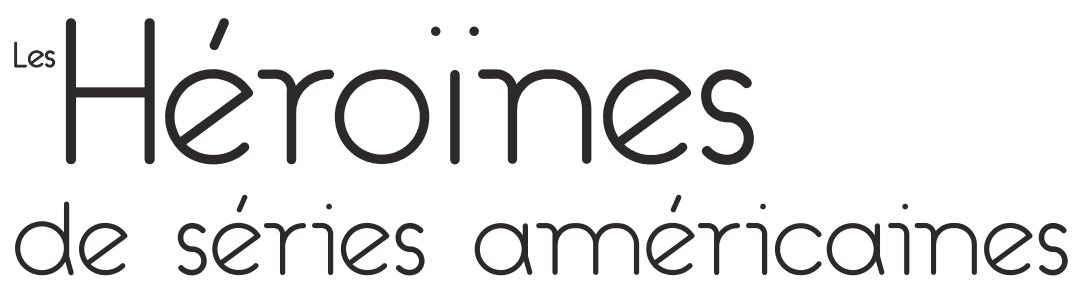


Dirigée par Stéphane Rolet, Sarah Hatchuel, Ariane Hudelet, Claire Cornillon et Éric Maigret, la collection « Sérial » s'attache aux manifestations du phénomène sériel sous toutes ses formes (littéraire, filmique, historique, sociologique, artistique, etc.), sans exclusive. Qu'elles soient plastiques (par exemple les séries de Warhol), littéraires (feuilletons, romans), ludiques (jeux vidéo, jeux de rôle), cinématographiques, télévisées, ou de bandes dessinées, les séries sont un phénomène de la modernité qui s'impose à nous en raison de leur nombre, de leur complexité et de la qualité artistique qu'elles peuvent atteindre. Dans une dynamique de pause et de reprise, la répétition du même et le principe de variation constituent de fait l'horizon d'attente de toute série, suscitant le plaisir sans cesse renouvelé de retrouvailles et de surprise. Loin de s'enliser dans une monotonie compulsive provoquant ennui et désintérêt, la série est devenue un phénomène de société, en particulier grâce à sa richesse d'invention. Bien que la série vise et promette un achèvement, elle peut cependant exister pleinement sans nécessairement avoir de fin. La série suppose aussi, très souvent, un mélange des genres fructueux et la migration d'un mode de création vers un autre : par exemple de la littérature vers les films ou à la série TV, de cette dernière vers la bande dessinée, ou vice versa. 
Céline Morin

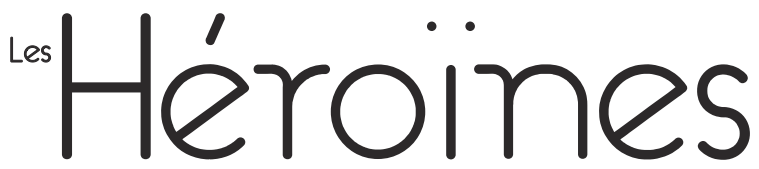

de séries américaines

de Ma Sorcière bien-aimée à The Good Wife

Presses universitaires François-Rabelais 
Conception graphique : Charlotte Boutreux • PUFR

Mise en page : Christine Martin • PUFR

(C) Tous droits réservés, 2017

Presses universitaires François-Rabelais

60 rue du Plat d'étain

BP 12050 • 37020 Tours cedex 1 • France

www.pufr-editions.fr

Dépôt légal : $2^{\circledR}$ semestre 2017

ISBN : 978-2-86906-491-1

ISSN : 2428-3231 


$$
\begin{array}{r}
\text { À Sienna, } \\
\text { la plus inspirante de mes héroïnes. }
\end{array}
$$





\section{Sommaire}

\section{Première partie - Amour, individualisme et courants féministes depuis 1950}

Égalités de genre, individualisme et amour 25

Le chaos amoureux de la deuxième modernité ou l'amour comme champ de bataille genrée - Individualisation, communication et socialisation : le casse-tête amoureux $•$ Du romantisme à la relation pure ?

Femmes et médias: courants de recherche 41

Une première étape dénonciatrice - Le bouillonnement épistémologique des film studies : sémiotique, psychanalyse et approches structurales de l'idéologie - La consubtantialité des hiérarchies culturelles et des hiérarchies de genre - Au-delà du fonctionnalisme et du structuralisme, vers le conflit de sens

Réconcilier l'individualisme et le féminisme et comprendre les subjectivités post-traditionnelles

Constructions et luttes internes du féminisme de la deuxième vague • L'ambiguïté des redéfinitions des corps par le néoféminisme • L'individualisme écartelé entre théories féministes et théories de la réflexivité - Dialogies entre tradition et post-tradition - Féminisme et individualisme sont-ils fatalement antinomiques ?

Du personnel politique à la politique individualiste - Un féminisme aux influences matérialistes encombrantes pour l'étude des imaginaires culturels ? • Un concept nébuleux pour une fracture générationnelle • Le postféminisme, courant postmoderne? 


\section{Deuxième partie - Un demi-siècle d'héroïnes de séries télévisées (1951-1997)}

Les conditions d'émergence des personnages féminins: des émancipations sous contrôle

Les femmes, un nouveau marché pour les annonceurs • Imaginaires du média et cohésion familiale - Une confusion de l'amour conjugal et de l'amour familial • Quand les femmes aiment aimer • Biopolitiques des femmes au foyer

Retour sur les premières héroïnes, suffragettes de l'ombre

L'intercompréhension comme remède à l'insatisfaction I Love Lucy, héroïne burlesque et premiers assauts émancipateurs ${ }^{\circ}$ L'amour domestique du Donna Reed Show - Ma Sorcière bien-aimée, émancipations contradictoires et subversions magiques

L'amour, meilleur ennemi des femmes actives

et des «féministes au foyer 》

La montée des subjectivités féminines • Dans The Mary Tyler Moore Show, être célibataire n’est pas être seule • Murphy Brown, affirmation féminine de soi et dominations amoureuses $\bullet$ La fin de l'abnégation féminine et le début des menaces castratrices $\bullet$ Les revendications contradictoires de Maude Roseanne : les amours en milieu ouvrier • Désenchantements quotidiens et microphysique des plaisirs

\section{Troisième partie - Les contradictions des héroīnes de l'après-féminisme (1997-2004)}

Expressivisme, post-modernité et après-féminisme 167

De «nouvelles "nouvelles femmes" » aux identités sensibles • La reféminisation des héroïnes : néoféminisme ou postféminisme ?• Tout avoir toute seule, le défi des héroïnes trentenaires • Controverses autour de la mort du féminisme dans Sex and the City et Ally McBeal

Fractures entre imaginaires, discours et pratiques 183

(Dé)constructions des amours • Labeur individuel, idéal de l'aisance romantique - Imaginaires idéalisés sur un modèle romantique concrètement affaibli • Dharma \& Greg ou la rare réussite démocratique 
Nouvelles subjectivités charnelles

et communications des désirs-plaisirs

Un nouveau levier identitaire • La sexualité, du soi au couple • Biopolitiques et technologies de soi à l'heure de l'émancipation féminine • La sexualité dans Buffy : entre apogée de l'amour maudit et expérience queer

\section{Quatrième partie - Le monde post-romantique des héroīnes quadragénaires (2004-2014)}

Détraditionnalisation, découverte du féminisme et urgence de nouveaux liens amoureux

Une émancipation malgré elles • Les responsabilités sont-elles solubles dans les psychotropes ? Culpabilités féminines et phénomènes compensatoires • Les ondes de choc de l'individualisme jusqu' à la maternité • Quelle communication amoureuse dans le post-romantisme?

Entre les désillusions du romantisme et les sables mouvants du mariage

Desperate Housewives : les émotions dangereuses $•$ Eros contre Thanathos dans The Big C : le sursaut féministe d'une mourante • Néoféministes à quarante ans $•$ Nurse Jackie ou l'androgynie des manières d'aimer

La rupture The Good Wife:

leçons de la première modernité, jeunesse de la modernité avancée et métamorphoses de l'amour

La fin du romantisme et les résistances du mariage bourgeois • Mariage en danger et dangers du mariage •Victoires et échecs d'une relation pure 



\section{Introduction}

Les héroïnes ne sont pas des passagères clandestines au pays des séries télévisées. Elles ne sont pas d'étranges singularités à qui l'on concéderait depuis peu un petit territoire en raison d'une supposée complexification des récits, de l'acceptation de l'« égalité des sexes » ou de politiques contemporaines de représentations des minorités. Les héroïnes sont, en réalité, un pilier originel de la télévision. On réalise finalement peu à quel point les personnages féminins ont participé conjointement du développement de cette forme médiatique toujours si populaire que sont les séries télévisées et de la diffusion des luttes féministes, en 1950 comme aujourd'hui. La télévision regorge depuis soixante ans de femmes au foyer soumises à l'insatisfaction de leur condition, de femmes actives cherchant dans la sphère professionnelle un refuge émancipateur que la maison n'a jamais pu être, de super-héroïnes rejouant le dilemme de la tragédie classique entre Amour et Devoir ou encore d'épouses trompées, prises au piège de leur abnégation, lançant une charge inédite contre leur propre couple.

Porter ainsi depuis sa genèse les traces du combat anti-sexiste, voilà ce dont aucun autre média ne peut se vanter. Ce livre est le fruit de quatre années de recherches et d'incalculables heures passées à coloniser les univers des séries télévisées, des univers luxuriants et complexes, chimériques ou désenchantés, tour à tour ou simultanément subversifs, conservateurs ou réactionnaires, mais toujours vecteurs de sens. La fiction n'est pas un monde de seconde zone condamné, au mieux, à répéter les dominations sociales ou, au pire, à encourager la dépossession de téléspectateurs fuyant leur quotidien. Cette vision matérialiste qui est celle de bien des théories féministes 
ne peut plus ignorer les travaux sur la force constructrice de l'imaginaire, du symbolique et du mythologique, au premier rang desquels se trouvent évidemment les écrits d'Edgar Morin. Or, les propositions mythologiques des séries s'inspirent précisément des combats féministes tout autant que des confrontations ordinaires entre hommes et femmes.

L'histoire des héroïnes de séries télévisées est indissociable de l'impératif de l'égalité de genre, bien sûr, mais aussi de la désillusion qu'a subie l'amour au cours du $\mathrm{XX}^{\mathrm{e}}$ siècle, et des reconfigurations qui suivent cette désillusion. Depuis l'avènement des idéaux égalitaires, lors des mouvements d'émancipation des femmes et des homosexuel.le.s, le romantisme apparaît comme engendrant des inégalités fortes. La fusion amoureuse est en fait le sacrifice des femmes pour la structure familiale, au profit d'un masculin à qui revient le privilège de l'épanouissement professionnel. L'affaiblissement des structures traditionnelles comme la famille charge l'amour d'un paradoxe. D'un côté, il est un des territoires, sinon le territoire, où sont le plus invisibilisés les rapports de pouvoir genrés (sous l'impulsion de la prédestination, les fameuses « âmes sœurs », les individus sont priés d'effacer les traces de la construction de l'amour, et par là même les rapports de pouvoir qui le construisent). D'un autre côté, il est devenu une modalité relationnelle promettant bonheur et épanouissement pour des partenaires soumis aux angoisses de l'individuation.

La collision entre émancipations féminines et amours, qu'il faut toutes deux mettre au pluriel tant les modèles varient et s'affrontent, produit des ondes de choc importantes, qui perturbent la distinction traditionnellement faite entre une sphère publique masculine (territoire de l'épanouissement professionnel et personnel) et une sphère privée féminine (royaume du relationnel et du communicationnel exhortant les femmes à l'épanouissement dans le sacrifice... injonction contradictoire s'il en est !). Cependant, si cette collision est explosive, elle est aussi créatrice. Les transformations évoquées peuvent encourager l'épanouissement individuel comme condition de l'épanouissement conjugal, prescrire un dialogue au sein du couple qui soit porté non plus vers la simple gestion du foyer mais vers l'intercompréhension des individus, et détacher la sexualité de sa fonction reproductrice pour en explorer les désirs et les plaisirs. La question se pose donc de savoir comment aimer, non pas après l'émancipation féminine, ce qui supposerait que celle-ci soit achevée, mais avec elle, alors 
même que tous ses objectifs n'ont pas été accomplis et que ceux qui l'ont été restent, comme toute victoire contre-hégémonique, très fragiles.

Les séries télévisées rendent compte de ces contradictions lorsqu’elles situent quasi systématiquement les femmes au cœur des affaires de cœur. Depuis plusieurs décennies, les héroïnes rejettent le couple traditionnel et recherchent de moins en moins le Prince charmant. Elles posent ainsi la question des nouveaux impératifs communicationnels dans des couples dont la légitimité n'est plus conditionnée au seul mariage. Cet ouvrage se donne pour but d'interroger ces représentations fictionnelles et d'étudier ce qu'elles décrivent des répercussions de l'émancipation féminine sur les structures conjugales, sur les manières d'aimer et sur les imaginaires amoureux. Une clé de voûte de cette recherche est le modèle de relation pure développé par le sociologue britannique Anthony Giddens, qui semble être le plus adapté à la compréhension des phénomènes contemporains en ce qu'il place l'égalité au centre de la quête amoureuse, dont la variété des formes doit être ramenée à l'impératif très contemporain de démocratie conjugale. Dans les imaginaires sériels, la relation pure devient un modèle de référence, certainement parce qu'elle permet de réarticuler le public et le privé par des processus interpersonnels de négociation et de compromis.

La reconfiguration des modèles amoureux n'est évidemment pas l'exclusivité de la télévision. Les conjonctures qui lient amours, émancipations féminines et médias de masse se sont construites et reconstruites dans la poésie, dans le roman ${ }^{1}$, au cinéma ${ }^{2}$ ou aujourd'hui sur Internet ${ }^{3}$, mais la télévision a ceci de particulier qu'elle a, dès ses débuts, accordé une large place aux représentations des inégalités de genre. Média familial par excellence, la télévision est particulièrement consommée par les femmes au foyer et elle a orienté en toute logique, y compris commerciale, ses récits vers les problèmes que renferme une sphère privée à priori dépolitisée. À ce titre, les séries sont donc des médiacultures ${ }^{4}$ participant aux luttes de définition du monde à partir de l'espace culturel et symbolique des représentations, et méritent d'être étudiées comme des « agents signifiants », selon la formule de Stuart Hall, qui sont à la fois dépendants des agencements idéologiques de leur contexte social et saturés de rapports de force hégémoniques et contre-hégémoniques. Répondant de surcroît à des logiques industrielles qui aspirent à contenter le plus grand nombre de consommateurs, elles sont structurées par le double principe éclairé par Edgar Morin d'une 
standardisation et d'une innovation ${ }^{5}$. Enfin, le public historiquement féminin ${ }^{6}$ de la télévision fait de ce média un terrain d'expression privilégié des problèmes de plus en plus prégnants d'égalité de genre dans le couple.

Cartographier les discours qui circulent dans les séries depuis soixante ans, c'est donc saisir des indices des redistributions du pouvoir entre les genres, en particulier sur le terrain amoureux devenu si sensible, c'est aussi comprendre le long et sinueux parcours de l'émancipation féminine, ponctué de pierres d'achoppement féministes et d'impasses patriarcales, rythmé par des offensives et des contre-offensives : culpabilisations, élans autoritaires, fuites ou encore exigences démocratiques. Cinquante ans après la publication de L'Esprit du temps, c'est encore se donner la chance de se poser la question de l'évolution du fonctionnement médiatique : les médias de masse permettent-ils toujours le jeu du renouvellement des représentations qu'Edgar Morin avait identifié ou laissent-ils davantage place aux effets de domination ? Ici, l'hypothèse est double : les séries télévisées semblent être le meilleur terrain pour interroger les nouveaux jeux d'équilibre entre conformisme et innovation dans les médias de masse, mais aussi pour saisir les rapports de force au sein des rapports amoureux contemporains, saturés de communication.

Avec ce syncrétisme qui caractérise les médias de masse, la télévision est donc un terrain privilégié pour qui veut comprendre les allants-desoi, les négociations et les rejets au sein des politiques amoureuses, selon le triple décodage emprunté à l'article désormais consacré « Codage/ Décodage » de Stuart $\mathrm{Hall}^{7}$, mais aussi les paradoxes et contradictions qui y alimentent de fortes luttes de signification, car Stuart Hall ne limite pas son modèle aux interprétations mais rappelle que le sens est avant tout conjoncturel, c'est-à-dire adossé aux politiques identitaires elles-mêmes complexes des récepteurs. Évitons donc de catégoriser trop sommairement les interprétations possibles et effectives, et n'hésitons pas à plonger, pour citer Edgar Morin, dans le « tissu complexe des relations sociales qui interfèrent dans le rapport émetteurs-récepteurs ${ }^{8} \gg$. Il y a là, il faut le souligner, une distinction importante entre cultural studies et gender studies : les cultural studies portent au cœur de leur épistémologie une attention toute particulière aux cultures populaires, qu'elles revalorisent comme médiatrices de luttes de sens. Tour à tour discréditées car consommées par des individus supposément dépourvus de recul critique ou par une 
masse dominante car artificiellement homogénéisée, les cultures populaires participent pourtant pleinement de la sphère publique : en visant à fédérer des publics hétérogènes, elles permettent d'instaurer un dialogue entre des idéologies contraires, produisant des représentations ambivalentes et syncrétiques ${ }^{9}$. Ce faisant, les cultural studies enrichissent la vision matérialiste qui est encore celle de nombreux champs féministes, ouvrant la voie à une analyse de la production d'imaginaires amoureux dans des imaginaires médiatiques. Dans les cultural studies, la télévision n'est donc pas un terrain d'étude annexé dans lequel guetter les reproductions des normes de genre et les tours de passe-passe plus ou moins discrets des dominants (ce qui ne revient pas à dire que ces reproductions et ces tours ne s'y produisent pas) mais elle est une empirie, au sens fort du terme.

En retour, l'approche en termes de rapports sociaux de genre est évidemment et indubitablement une alliée dans la compréhension des rapports de pouvoir entre hommes et femmes à la télévision. Quelles places les séries télévisées confèrent-elles historiquement aux héroïnes ? Quelles sont les interactions entre identités de genre et contrats amoureux ? Que produisent les relations de causes à effet, les rapports de force et les négociations, entre des pôles divers mais articulés comme le féminisme et la féminité, le féminisme et l'individualisme ou encore le féminisme et la sexualité ? Toutes ces questions trouvent une entrée décisive dans la formalisation du genre comme rapport social. Cependant, l'approche du genre ne peut être une alliée qu'à condition d'accepter de se pluraliser. Si Judith Butler, la première, évoque les rapports de genre au singulier, la perspective queer qu'elle propose fait proliférer les genres pour montrer leur aspect construit. Or, les performances de genre sont en effet plurielles dans les séries : performances masculines par exemple chez Murphy Brown, performances néoféministes dans Sex and the City, performances stoïques dans The Good Wife. Le genre comme rapport social n'est pas, ne doit pas être, dissocié des genres en tant que performances collectives ou individuelles. S'ouvrir à leur diversité permet de dépasser une vision aux tendances structuralistes qui a le mérite d'éclairer les impasses mais dont l'écueil est d'appauvrir voire de rejeter le potentiel reconfigurant de la culture populaire, faute en réalité d'outils adéquats pour la comprendre. Cela est d'autant plus pressant dans un domaine de représentations comme la télévision, qui repose aussi solidement sur le symbolique. Aussi trouvera-t-on, tout au long de cet ouvrage, une analyse 
des héroïnes dans leur polyphonie : à partir des années 1990 principalement, même si l'on peut trouver des germes de ce mouvement dans les décennies précédentes, une fragmentation des identités féminines représentées se produit. Les personnages deviennent des « sujets nomades » selon la belle formule de Rosi Braidotti ${ }^{10}$. Parce qu'elles ne sont plus simplement déterminées par les «oppositions dialectiques et des dualismes structurants », ces hérö̈nes ne s'ancrent plus de façon simple dans un rapport de genre au sens masculin-féminin. Et même si la dénaturalisation du genre est encore balbutiante - sans parler même de sa déconstruction!-, cela permet déjà de voir des bouleversements forts : réappropriation des corps et de la sexualité, androgynisation des manières d'aimer par l'abandon du sacrifice, ou encore colonisation de la sphère professionnelle. Cette recherche porte donc sur la pluralisation des sujets féminins, et non sur la mise en relation généralisée de genres supposés immuables.

Ce sont peut-être, à bien des égards, les « contre-publics subalternes » qu'il faut convoquer ici, contre-publics dont les stratégies ne sont pas forcément progressistes. La structuration de la sphère publique au sens de Nancy Fraser, faite de compromis démocratiques, s'insère en effet dans les espaces privés : à mesure que se décompose ce qu'Ulrich Beck appelle le « cœur féodal de la société ${ }^{11}$ » qu'est la division des tâches en fonction de la dichotomie masculin/féminin, les batailles se déplacent vers le territoire conjugal. Nancy Fraser signale que les féministes ont importé dans la sphère publique des problématiques jusqu'ici disqualifiées par leur confinement à la sphère privée. Elle rappelle avec force que les définitions traditionnelles de ce qui est « privé » créent une « rhétorique de l'intimité qui, historiquement, a été utilisée pour restreindre le champ de la contestation publique légitime ${ }^{12}$ ». La constitution des femmes en « contre-public subalterne » s'est faite dans le domaine public sous la forme, notamment, de mouvements féministes militants, mais elle peut tout autant être scrutée dans la production industrielle de personnages féminins : les héroïnes de séries télévisées, à leur manière, élaborent de plus en plus des « arènes discursives parallèles dans lesquelles, [en tant que] membres des groupes sociaux subordonnés, [elles] élaborent et diffusent des contre-discours, afin de formuler leur propre interprétation de leurs identités, leurs intérêts et leurs besoins ${ }^{13} \gg$. Lorsqu'elles discutent entre elles des inégalités vécues ou lorsqu'elles confrontent leur partenaire à leurs insatisfactions, ces héroïnes 
expriment, à partir de l'expérience féminine telle que la construit la fiction étasunienne, des propositions de redistributions du pouvoir.

Il n'échappera pas au lecteur que cet ouvrage privilégie un corpus portant exclusivement sur les séries télévisées étasuniennes. Ce choix s'explique par un contexte d'industrialisation poussée qui stimule la production d'imaginaires certes, selon Edgar Morin, standardisés mais aussi profondément innovants. Sont sélectionnées vingt-deux séries diffusées de 1951 à 2010, dont le personnage principal est une femme hétérosexuelle. Dans les séries télévisées, les régimes hétérosexuels apparaissent en effet comme les plus à même de résister à la représentation d'une compréhension inter-genre, c'est-à-dire à une démocratie relationnelle basée sur l'écoute de différences qui, pour autant qu'elles n'ont évidemment pas de prises « naturelles » mais naturalisées, produisent des effets bien réels dans les représentations médiatiques des genres.

Trois pôles structurent l'interprétation de ces représentations médiatiques. Premièrement, le symbolisme du genre (féminin/masculin) et la modélisation de l'amour à travers ce symbolisme permettent de comprendre la façon dont les communications (compromis, renoncements, négociations, ultimatums, etc.) sont rendues plus ou moins saillantes selon les genres. Deuxièmement, la division sociosexuelle du travail, c'est-à-dire le jeu entre sphère publique et sphère privée, est analysée à l'aune des modèles féministes et des stratégies d'émancipations. Troisièmement, la construction de l'identité individuelle de genre est scrutée selon les subjectivités données aux héroïnes : rapport au verbal, au corps, à la sexualité, aux techniques de soi mais aussi aux anxiétés ou addictions en tout genre. Ces trois niveaux ne se co-déterminent pas ni ne produisent une interprétation univoque. À contrario, la complexité de la représentation d'héroïnes aux prises avec l'égalité de genre dans l'amour ne peut être comprise qu'en articulant ces trois axes : types de féminisme, types de modèles amoureux et types de subjectivités.

Ce livre comprend quatre parties. Après être revenue sur les débats épistémologiques relatifs à l'amour et aux rapports entre femmes et médias, seront abordées les phases de développement des héroïnes de séries télévisées découpées elles-mêmes en quatre périodes : de 1951 à 1970 ; de 1970 à 1997 ; de 1997 à 2004 et de 2004 à 2014 (les séries ayant débuté après 2010, soit le début de cette recherche, ne sont pas prises en compte). 
La première partie explore les liens entre amours et féminismes. À rebours de certaines propositions essentialistes présentes dans la philosophie ou la psychanalyse, l'amour y est défini comme une modalité relationnelle individuellement modulée et structurée par les genres. En même temps que décroît l'idéologie du sacrifice personnel et que se forge une démocratie relationnelle où chacun doit en théorie pouvoir se faire entendre, l'individualisme réflexif et le féminisme sont mobilisés pour définir l'amour comme une visée communicationnelle. Pour pleinement comprendre les politiques représentationnelles des héroïnes, il faut donc également revenir sur la construction hétérogène des mouvements féministes et leurs liens souvent conflictuels avec l'individualisme réflexif. Les différentes formes de féminisme des années 1960 et 1970 ne peuvent survivre aux mutations sociales qu'elles ont elles-mêmes engendrées ou qui proviennent des mouvements d'individualisation sans actualiser leurs objectifs et leurs propositions : le défi de la réinvention de l'amour apparaît devoir s'accompagner d'une réinvention des féminismes, cela sans balayer un individualisme réflexif précieux pour l'affirmation des identités et des politiques. Ce livre défend donc la possibilité d'une réconciliation entre féminisme et individualisme réflexif, dont on trouve d'autant plus de traces dans les séries télévisées qu'elles privilégient précisément l'incarnation des controverses sociales dans des héros individualisés (désormais, des héroïnes).

Une fois ce cadre posé, la deuxième partie opère un retour historique d'un demi-siècle, couvrant les deux premières époques de l'histoire des héroïnes de séries télévisées : parce que la compréhension des représentations contemporaines ne peut être pleinement comprise qu'en la situant diachroniquement, cette recherche débute avec ce qui est communément considéré comme la première sitcom de la télévision étasunienne, I Love Lucy (1951-1957). La première période, de 1950 à 1970, met en scène l'euphorie domestique, orchestrée par des femmes au foyer gaiement soumises, à l'instar de The Donna Reed Show. Quelques hérö̈nes viennent perturber cette tranquillité domestique typique des imaginaires étasuniens de l'aprèsguerre, comme Lucy Ricardo, qui passe son temps à fuguer du foyer, ou Ma Sorcière bien-aimée, balançant sans cesse entre l'exercice de ses pouvoirs, métaphore de ses capacités d'agir féminines, et leur abandon au profit d'un travail domestique manuel, sous-tendu par l'idéologie méritocratique, parfaitement inséré dans le fonctionnement de la famille nucléaire. 
Une seconde période, de 1970 à 1997, a tendance à reproduire la division initiale entre féminisme libéral et féminisme radical. Le féminisme libéral est incarné par des working women comme Mary Richards et Murphy Brown, qui voient dans la conquête de la sphère publique le levier émancipatoire des femmes - tout en en interrogeant les limites dans le désert amoureux qu'elles subissent en retour. Le féminisme radical est défendu par ce que l'on pourrait nommer des «féministes au foyer » : des épouses et mères de famille, comme Roseanne ou Maude, qui luttent pour faire entendre leur voix au sein du foyer dont elles critiquent l'organisation patriarcale.

La troisième partie retrace la période partant de 1997 (le point de départ en étant Ally McBeal) à 2004, qui voit surgir des héroïnes plus solitaires (Charmed, Gilmore Girls, Alias, Sex and the City, Ally McBeal), souvent trentenaires, marquées par le féminisme et négociant romantisme et relation pure dans une confrontation plus globale entre épanouissement professionnel et bonheur amoureux. Ces héroïnes tentent désespérément de réinvestir la sphère privée après avoir brillamment conquis la sphère publique. Elles symbolisent ainsi l'accomplissement du féminisme tandis que leurs névroses et leurs malheurs somment de repenser le public et le privé ensemble et non l'un après l'autre : le problème n'est plus de conquérir la sphère publique qu'elles ont investie et qu'elles ne remettraient jamais en cause (ce qui les différencie des héroïnes antiféministes), mais de voir les luttes émancipatrices se réfugier dans la sphère privée et notamment dans la structure du couple. La fracture qu'elles activent entre des pratiques d'amours proches de la relation pure et des imaginaires romantiques est caractéristique d'un syncrétisme médiatique qui produit ici le paradoxe de femmes apparemment émancipées de tout sauf du Prince charmant. Ce dernier semble être le prix à payer pour la monstration d'héroïnes fortes - signe des échecs du féminisme radical.

Enfin, la quatrième partie révèle l'apparition massive, à partir de 2004, de femmes quadragénaires incarnant une certaine vision des cinquante années d'émancipation féminine. Souvent veuves ou divorcées avec enfants, ces héroïnes ont un avantage certain sur leurs précédesseures puisqu'elles ont accompli les attendus sociaux (mariage, enfants) et rassuré la norme hétérosexuelle. Elles n' interrogent pas l'après-féminisme comme les hérö̈nes de la période précédente mais l'après-romantisme : tandis que les personnages trentenaires pratiquaient des relations pures dans l'espoir de les transformer 
en relation romantique, cette nouvelle vague d'héroïnes trouve dans la déception du romantisme, déception explicitement causée par des inégalités de genre, le moteur pour accomplir des relations plus égalitaires. Se produit alors une critique systémique de l'organisation domestique patriarcale et une responsabilisation - enfin! - des personnages masculins. Dans ces séries, la dimension structurelle de l'oppression est réintégrée d'une façon qui prend en considération les nouvelles porosités entre sphère publique et sphère privée et le potentiel émancipateur de l'individualisme.

\section{Notes}

Ainsi que l'ont montré les travaux de Denis de Rougemont, Niklas Luhmann ou Jean-Claude Kaufmann.

2 Je renvoie à Stanley Cavell et David Shumway.

3 Voir Eva Illouz ou Jean-Claude Kaufmann.

4 Maigret, Éric, Macé, Éric, Penser les médiacultures. Nouvellespratiques et nouvelles approches de la représentation du monde, Paris, Armand Colin, 2005.

5 Morin, Edgar, L'Esprit du temps, Paris, Armand Colin, 2010.

6 Spigel, Lynn, Make Room for TV. Television and the Family Ideal in Postwar America, Chicago, University of Chicago Press, 1992.

7 Hall, Stuart, « Codage/décodage », in Glevarec, Hervé, Macé, Éric, Maigret, Éric, Cultural Studies. Anthologie, Paris, Armand Colin, 2008.

8 Morin, Edgar, L'Esprit du temps, Paris, Armand Colin, 2010, p. X.

9 Morin, Edgar, L'Esprit du temps, Paris, Armand Colin, 2010.

10 Braidotti, Rosi, « Les sujets nomades féministes comme figure des multitudes », Multitudes, 12, 2003.

11 Beck, Ulrich, Beck-Gernsheim, Elizabeth, The Normal Chaos of Love, Cambridge, Polity Press, 1995, p. 25.

12 Fraser, Nancy, « Repenser la sphère publique : contribution à la critique de la démocratie telle qu'elle existe réellement », Hermès, 2001, 31, p. 125-157.

13 Fraser, Nancy, ibid. 
Amour, individualisme et courants féministes depuis 1950 

La richesse des imaginaires médiatiques relève de leur syncrétisme, ce à quoi n'échappent pas les influences féministes des séries télévisées. Au cours de l'histoire des hérö̈nes étatsuniennes, les courants varient et se contredisent : féminisme libéral, féminisme radical, néoféminisme et postféminisme forment le spectre idéologique de ces séries. Ce chapitre propose de réinscrire la complexité des relations représentées entre les femmes et les courants féministes dans le contexte de la détraditionnalisation et du passage de la première à la deuxième modernité. Par ailleurs, loin des constatations éplorées des auteurs critiques (Badiou, Hardt \& Negri, Illouz, Žižek) pour qui l'amour a été corrompu par la modernité capitaliste et par l'individualisme, une démarche compréhensive à l'égard des relations amoureuses permet d'éclairer autrement leur labilité contemporaine dont chacun.e fait l'expérience dans les sociétés occidentales, et qui inspirent les scénaristes et producteurs de séries télévisées.

Pour démêler ces paradoxes, il faut revenir tout d'abord sur les dernières propositions de la sociologie quant à l'amour, avant d'insister sur la pluralité des courants féministes ainsi que sur le potentiel humaniste et égalitariste de l'individualisme. Quelques-uns des concepts cruciaux employés pour analyser les héroïnes de séries télévisées amalgament des objets, des théories et des idéologies très différentes : pour certain.e.s chercheur.e.s, l'individualisme est atomisation et dépolitisation, pour d'autres, il est micropolitique et humanisme; pour certain.e.s, le postféminisme est un antiféminisme individualiste, pour d'autres, il est l'affirmation d'un sujet autonome et responsable; pour certain.e.s, le néoféminisme est ré-essentialisation du genre, pour d'autres, il est réappropriation des corps. C'est donc la mise en perspective des articulations historiques entre régimes de modernité, courants féministes, contrats amoureux et propositions mythologiques des médias de masse qui construit ce chapitre. Lorsque les séries télévisées prennent acte de l'émancipation des femmes, l'amour est-il redéfini comme un eldorado perdu (la famille nucléaire n'est plus accessible aux femmes actives) ou comme un territoire à reconstruire ? Reste-t-il à la charge du féminin ou sa responsabilité est-elle redistribuée entre hommes et femmes ? Face à l'anxiété que produit l'émancipation, ses modalités d'exercice s'enracinent-elles toujours dans un idéal romantique fusionnel ou se diffusent-elles dans des registres démocratiques ? Plutôt que de considérer les héroïnes individualistes et féministes comme un contresens, il faut s'interroger sur ce que ces nouveaux profils, certes ambigus, parfois même contradictoires, font subir aux performances de genre et aux liens sociaux des héroïnes. 



\section{Égalités de genre, individualisme et amour}

\section{Le chaos amoureux de la deuxième modernité ou l'amour comme champ de bataille genrée}

Parce que les sociétés traditionnelles ont laissé place aux sociétés industrielles et parce qu'à leur tour ces sociétés industrielles se désagrègent, les individus sont désormais en charge de la construction du sens et de l'organisation du monde, sans pouvoir s'appuyer solidement sur les structures, institutions et traditions devenues fragiles. Le rôle de l'émancipation féminine dans cette redéfinition n'échappe pas à Ulrich Beck et Elisabeth Beck-Gernsheim ${ }^{1}$ qui font intervenir cinq variables : l'allongement de la durée de vie des femmes et son corollaire, la complexification de leur biographie qui intègre désormais un « après-enfants », la révolution ménagère, le développement de la contraception et de l'avortement, la légalisation du divorce et l'égalisation des conditions d'accès à l'enseignement supérieur. Comparant les rôles genrés avec les distinctions de classe, Beck et Beck-Gernsheim constatent un redéploiement des conflits sociaux dans la sphère privée. Dans cette modernité avancée, l'amour qui n'est plus régi par la famille ou l'économie a pour fonction charnière de générer l'espoir d'un épanouissement personnel. Après avoir été la construction d'une vie en équipe, la relation amoureuse se fait désormais entre deux partenaires, chacun étant pour l'autre à la fois amant et ami. Religion séculière, l'amour est envisagé comme le moyen d'atteindre le Saint Graal moderne, le bonheur. « Saint Graal » car, selon Beck et Beck-Gernsheim, l'amour est devenu «la croyance ultime après la fin de toutes les fois ${ }^{2} »$, 
religion après la religion. Sa fonction est de contrer les désagréments de l'individualisme en ce qu' il est une nouvelle forme de lien social permettant une « stabilité liée à la personne ${ }^{3}$ » (person-related stability). Parce qu'il ne peut plus se reposer sur une stabilité structurelle, l'individu cherche en l'autre l'aide nécessaire pour se définir, trouver sa place, élaborer son bienêtre. Beck propose ici peut-être la définition fonctionnelle la plus satisfaisante de l'amour, celle d'une stabilité (qui n'est pas forcément immuable) émotionnelle et mentale calée sur le soutien d'autrui et donnant «à la vie sa substance et sa signification ${ }^{4} \gg$. Si la quête du bonheur n'est pas nouvelle, cet investissement dans l'amour teinté de religion est un phénomène très contemporain. L'analogie de Beck ne s'arrête pas au bonheur que promettent amour et religion mais compare aussi les chemins pour y parvenir : en échappant à la trivialité du quotidien ou en lui conférant une aura nouvelle, en produisant de nouvelles significations et de nouvelles réalités, l'amour crée un nouveau monde dans lequel l'individu peut théoriquement se réfugier pour être lui-même, authentique. D'autres sociologues ont éclairé les résidus religieux dans l'amour, à la manière surtout de Jean-Claude Kaufmann, confronté à l'importun Prince charmant sans cesse re-convoqué par des femmes pourtant émancipées. La « non-tradition ou post-tradition » de l'amour promet du lien social et donne sens à la vie, elle devient aussi le lieu où les personnalités se définissent et se redéfinissent, se cherchent, se trouvent et se fuient, se comprennent ou se négocient. Aux réflexions de Beck, ajoutons que s'il y a dans l'instabilité moderne les germes d'une solitude existentielle, ces infinies possibilités de réalisations personnelles suscitent aussi une excitation trop souvent balayée par les théoriciens critiques. La construction du sens qu'identifie bien Beck peut être angoissante tout comme elle peut être un plaisir. Dans un monde rationalisé, l'amour tire son attrait de cette capacité à recouvrir d'un voile les processus techniques et réfléchis mais, comme les religions qui l'ont précédé et l'ont construit, l'amour perd aujourd'hui de sa force mythologique et se rationalise aussi. Dans un élan prophétique, Beck prédit l'émergence d'une forme d'amour calculable, soutenue par les systèmes juridiques et la médecine, notamment l'ingénierie génétique. Dans le même temps, non sans contradiction, il n' imagine pas que le modèle traditionnel puisse être remplacé par un seul autre, mais imagine plutôt la cohabitation d'infinies combinaisons et de multiples pratiques. 
Il est finalement impossible de donner un même terme à toutes les pratiques englobées dans le terme d'«amour ». La multiplicité des formes amoureuses contemporaines et ses corollaires - espoir, trahison, désir, jalousie - sont la résultante de l'effritement de la famille nucléaire et des rôles genrés qu'elle a construits, elles sont aussi la conséquence des émancipations et des droits acquis. Dogmatisme pour deux qui échappe à l'institutionnalisation et à la codification, distribué de façon inégale et inégalitaire, l'amour valide l'identité selon Beck et Beck-Gernsheim, déjoue la solitude et le doute. Il ne repose pas sur des comportements rationnels et instrumentaux, il n'est pas non plus un but en soi mais un moyen d'atteindre le bonheur. Perméable à l'incertitude et à la réflexivité modernes, son épanouissement est la responsabilité de sujets actifs et non de structures traditionnelles. Démocratique, donc, mais aussi autoréférentiel puisqu'émotion justifiée par l'émotion, l'amour serait une religion séculière, la promesse d'un refuge heureux dans une modernité avancée caractérisée par le risque. Le monde sécularisé a en effet trouvé en l'amour un nouveau Dieu en réalité très ancien, mais les modalités contemporaines de son règne sont, elles, en revanche bien nouvelles.

Modèle amoureux de référence depuis le XviII siècle en Occident, le romantisme est donc confronté depuis le milieu du $\mathrm{Xx}^{\mathrm{e}}$ siècle aux asymétries profondes qu'il provoque entre hommes et femmes. De nouvelles pratiques affleurent, qui trouvent un écho médiaculturel, et qui placent en leur cœur une communication conjugale visant à respecter les individualités, non plus sommées de se liquider dans le couple, mais envisagées comme constitutives du couple.

Pour comprendre ces mutations, il faut revenir aux fondements du romantisme. Plusieurs évolutions sociohistoriques ont précédé et encouragé l'avènement de ce modèle : la création du foyer familial, l'évolution des relations entre parents et enfants, l'invention de la maternité sont autant de mutations opérées au cœur de la vie privée, et plus particulièrement au cœur de la vie privée des femmes, qui sont décisives dans la constitution du romantisme, alors modèle émancipateur. On ne peut donc ignorer, comme le rappelle Anthony Giddens, les ambitions égalitaristes « contenues dans l'idée qu'une relation amoureuse est susceptible de découler du seul engagement émotionnel de deux individus plutôt que de critères sociaux extérieurs $^{5} \gg$, mais l'on ne peut pas non plus méconnaître ses inégalités genrées. 
Ses modalités relationnelles, au premier rang desquelles la fusion, entérinent des rôles sociaux et des prédispositions comportementales comme l'amour oblatif qui tendent à sacrifier le féminin plutôt que le masculin - ce qui explique en retour son affaiblissement contemporain lorsqu'il affronte de plein fouet les nouveaux idéaux égalitaires hommes/femmes. C'est peut-être dans le modèle romantique que s'expriment aujourd' hui le plus fortement les contradictions entre imaginaires et pratiques : Jean-Claude Kaufmann a raison de dire que « nous sommes encore des héritiers directs du romantisme dans nos manières d'aimer ${ }^{6} \gg$, ce que cristallise bien la figure du Prince charmant, mais les assauts émancipateurs de la deuxième modernité ont très largement minimisé les pratiques romantiques concrètes.

Si l'amour romantique a permis pour la première fois d'articuler des antagonismes comme l'amour et la liberté en proposant à l'individu de s'émanciper des carcans familiaux traditionnels, d'autres chaînes ont été retrouvées, dues à une division des sphères publique et privée qui assignait les hommes à la première et les femmes à la seconde. Aussi est-il, comme l'avaient déjà montré les universitaires féministes, « difficile de nier que les idées ayant trait à l'amour romantique contribuèrent à la position de dépendance de la femme au sein du foyer et à sa relative séparation visà-vis du monde extérieur ${ }^{7} \gg$. Non sans contradiction, cette assignation est ce qui a permis aux femmes d'exprimer leur capacité d'agir. « Sousprolétariat émotionnel » pour Anthony Giddens ou « agents secrets » de la modernité pour Edgar Morin, les femmes, portées par des compétences sentimentales que leur assignation à la sphère privée leur a permis de développer, se sont attaquées à ce qui étouffait leur individualisme réflexif et ont provoqué un chamboulement de l'ordre amoureux dans la deuxième moitié $\mathrm{du} \mathrm{xx}^{\mathrm{e}}$ siècle. L'identification projective, la perte du soi dans le couple et la quête de transcendance, parce qu'elles étaient censées être la particularité des femmes plus que des hommes, cachaient des rôles genrés qui entérinaient des dominations notamment domestiques comme la dépendance des femmes au foyer. Ce mouvement égalitaire de la deuxième moitié du $\mathrm{Xx}^{\mathrm{e}}$ siècle, éclairant le prix genré de l'amour romantique, est force de transformations.

Dans Love in America. Gender and Self-Development ${ }^{8}$, Francesca Cancian articule une thèse brillante autour de la distinction entre sphère privée et sphère publique, apparue au cours du $\mathrm{XIX}^{\mathrm{e}}$ siècle et qui aurait créé un 
conflit entre amour et développement personnel. S'est en effet établi un système oppositionnel entre, d'un côté, une maisonnée source d'amour féminin, et de l'autre, une vie professionnelle masculine, lieu d'épanouissement personnel. Sur la base d'un syllogisme, privé et public étant opposé, féminin et masculin étant opposé, amour et développement personnel sont également perçus comme contradictoires. L'affection féminine doit alors se baser sur l'oubli de soi, le sacrifice. Cancian en conclut que l'opposition bien connue entre liens familiaux (ou amoureux) et épanouissement individuel ne pourra être résolue que lorsque celle entre amour féminin et développement masculin le sera. Relisant l'historienne Mary Ryan", Francesca Cancian rappelle les processus de « féminisation de l'amour ${ }^{10}$ » et en étudie les conséquences contemporaines. Contre cette opposition qu'ont créée les chercheurs entre épanouissement individuel et engagement dans le couple, elle montre l'interdépendance contemporaine de ces deux éléments, extrayant de ses recherches empiriques la nécessité d'être individuellement épanoui et d'avoir un partenaire qui le soit également pour qu'une relation amoureuse soit réussie. À partir de ce constat, Cancian fait une critique aiguisée des sociologues qui regrettent le déclin des communautés comme Christopher Lasch ou Robert Bellah, pour qui le prix à payer de la liberté individuelle est l'affaiblissement des liens sociaux, lequel en retour annihile le développement personnel. Elle montre, au contraire, à quel point ces théoriciens exagèrent l'affaiblissement des liens sociaux, sous-estiment le prix de l'autorité patriarcale, et sont nostalgiques d'un passé qu'ils reconstruisent comme romantique.

Suivant la tradition féministe des années 1970, elle fait une analyse matérialiste de cet amour ainsi féminisé, défini par la tendresse, l'expression des émotions, la vulnérabilité et reposant sur une assignation des femmes à la famille : cette distinction prendrait source dans la mutation sociale d'une économie agraire vers une économie capitaliste, poussant les hommes hors du foyer familial et encourageant une division des tâches, donc des rôles. Pour Cancian, un modèle androgyne de l'amour apparaît donc dans les années 1970 lorsque l'épanouissement personnel devient l'objectif premier des individus, masculins comme féminins, lequel modèle tente l'articulation de l'indépendance masculine et de l'affection féminine, rendant les sphères plus poreuses et les rôles plus dépendants des envies que des genres - ces derniers continuant bien sûr de produire des déterminations sociales. 
Cancian définit ainsi l'amour comme une relation interdépendante dans laquelle s'expriment l'affection, l'acceptation, le soin, l'attention, l'engagement et qui donne priorité à l'être aimé. Cette définition, qui jusqu'ici peut également désigner les relations amicales et familiales, s'enrichit d'une intimité sexuelle, d'une affection physique et d'une coopération domestique quotidienne exclusives aux relations amoureuses. In fine, ces qualités doivent servir le développement personnel mutuel, la communication et l'intercompréhension et que doit aider une souplesse des rôles genrés - des éléments que l'on retrouve dans le concept plus affiné de « démocratie relationnelle »d'Anthony Giddens. La féminisation de l'amour n'est donc pas que normes comportementales et assignations genrées. Elle a également touché la définition même de l'amour, jusque dans les travaux scientifiques : ayant pour pierre angulaire l'expression des sentiments, « ces définitions contemporaines de l'amour mettent clairement l'accent sur des qualités considérées comme féminines ${ }^{11} \gg$, niant par contrecoup l'expression masculine des émotions souvent plus pratique - Cancian rapporte les efforts d'un époux qui, pour signifier son amour, lava la voiture de son épouse et fût très surpris de constater que son geste n'ait pas été considéré comme affectueux. Une conséquence très contemporaine de cette définition féminine qui refuse d'inclure les caractéristiques supposées masculines est une alliance conflictuelle entre amour et sexe, comme l'identifie très bien Cancian :

« Parce que l'intimité sexuelle est la seule façon "masculine” d'exprimer l'amour qui soit reconnue culturellement dans notre société, la récente tendance à considérer la sexualité comme une façon pour les hommes et les femmes d'exprimer mutuellement l'intimité est une contestation majeure de la féminisation de l'amour ${ }^{12}$.

Loin d'être un moyen pour les hommes de dominer les femmes comme a pu l'avancer Eva Illouz, la réappropriation de la sexualité par ces dernières est au contraire un formidable signe d'émancipation féminine et d'une androgynisation de la définition de l'amour - une redéfinition dont on peut imaginer les répercussions égalitaires dans les pratiques, tant en termes de droit et de liberté que de communication conjugale. 
Plusieurs auteurs ont ainsi noté à partir de la deuxième moitié du $\mathrm{Xx}^{\mathrm{e}}$ siècle une transformation de l'intimité et des rapports amoureux portée par l'individualisme réflexif, par les mouvements émancipateurs des femmes et des homosexuel.le.s, et par la libération de la sexualité. Ce constat est décisif pour saisir les redéploiements communicationnels dans l'amour. Que ce soit sous les traits d'une « liquidité ${ }^{13} \gg$, d'un « amour fissionnel ${ }^{14}$ », d'un « chaos amoureux ${ }^{15}$ » ou d'une « pureté des relations ${ }^{16} \gg$, est analysée la liberté d'entrer dans, et de sortir d'une relation amoureuse, mais aussi de la construire selon les logiques individuelles (envies, besoins, attentes, craintes).

Sur les mutations de l'intimité, Zygmunt Bauman n'échappe pas à un constat en forme de regrets : la sexualité libérée et la relation pure sont des victoires à la Pyrrhus où les individus se contentent de moins. Les « relations de poche » qu'il décrit, prudentes et éphémères, s'ancrent dans une modernité où le contexte est flottant. Cette « liquidité » amoureuse trouve sa formalisation la plus précise dans l'analogie physique par laquelle Serge Chaumier oppose au processus romantique de la fusion une fission amoureuse basée avant tout sur le respect des individualités. Ce courant, émaillé d'éloquentes formules de liquidité ou de fission, a le mérite d'identifier les tactiques de sécurisation des espaces personnels. Loin de souscrire aux descriptions inquiètes de Bauman et encore moins aux formulations parfois essentialisantes et souvent moralistes de certains chercheurs, Chaumier photographie, en négatif de la fusion amoureuse, un modèle mi-descriptif mi-prophétique des évolutions amoureuses de ces dernières décennies. Ce modèle de la « fission » est respectueux de la liberté des partenaires, une liberté plus proche de l'indépendance que de l'autonomie, et entend être capable d'intégrer l'hétérogénéité des pratiques en ne dictant pas de contrat particulier, à l'inverse du modèle passionnel ou romantique. La relation fissionnelle n'a donc pas l'exclusivité ni l'ouverture sexuelle pour clé de voûte, mais la possibilité d'exister en dehors du couple, une indépendance qui mène à constituer et/ou à préserver des liens amicaux ainsi que des temps et des espaces distincts. Le couple est « plutôt une ancre qui sert de point de repère pour aller à la rencontre des autres ${ }^{17} \gg$, tout l'inverse du huis clos prescrit par le romantisme et la passion. Car, à bien lire L'Amour fissionnel, le pivot de la théorie de Serge Chaumier semble n'être pas tant l'amour que l'identité. Son analyse porte souvent 
sur la pluralité des identités qu'illustre bien le terrain amoureux et elle peine à s'extirper de la constatation (au demeurant tout à fait juste) d'une incroyable hétérogénéité des pratiques à laquelle l’hégémonie romantique ne rend pas honneur. À vouloir englober tant de pratiques différentes et contradictoires, la catégorie d'《 amour fissionnel », si utile pour remettre en cause l'hégémonie romantique, se révèle néanmoins plus nébuleuse que définitionnelle. La perspective de Serge Chaumier est audacieuse en ce qu'elle va au-delà des définitions sociologiques classiques qui perçoivent l'amour comme un producteur de lien social. Pour l'auteur, il est avant tout producteur de liens sociaux, au pluriel, qui sont au service de l'identité personnelle et par rapport auxquels le couple est un point de repère pour l'individu vagabond. On se réjouit d'une description qui laisse place à une autonomie relationnelle et à l' élaboration d'une vie sociale qui ne relève pas d'un jardin secret souvent culpabilisant, mais on peut aussi s'interroger, au vu surtout des apports kaufmanniens en la matière, sur la difficulté qu'a Serge Chaumier à intégrer dans sa lecture parfois postmoderne les joies d'un quotidien amoureux que d'aucuns peuvent juger aliénantes mais qui n'en sont pas moins réelles. L'intéressante opposition entre un amour fusionnel et un amour fissionnel traduit le glissement d'un couple exclusif et autosuffisant vers des amours relationnelles. L'apport queer est sûrement ce qui permet aux travaux de Serge Chaumier d'être la première réflexion française sérieuse sur le « polyamour » qui ne soit pas teintée d'affolements moralistes, cependant la construction d'un ennemi principal, le romantisme, s'alourdit d'une simplification de ce courant alors résumé à son autoréférence constante qui oublierait les variations individuelles. Loin de défendre l'amour romantique dont les jeux de pouvoir genrés ne sont plus à démontrer, il faut tout de même rappeler son histoire émancipatrice et sa capacité à admettre des contraires et des contradictions (ce que Kaufmann a bien montré en éclairant l'étrange alliance entre femmes indépendantes et princes charmants). Parce que les descriptions du romantisme proposées par Serge Chaumier sont par ailleurs particulièrement pertinentes, le problème semble résider dans sa seule prise en compte de l'imaginaire romantique au détriment des pratiques romantiques qui souvent le contredisent. C'est donc un type de romantisme très précis saisi ici, celui qui ambitionne d'allier imaginaires et pratiques romantiques mais qui n'a pour ainsi dire jamais trouvé une réalisation stable et durable, comme Chaumier d'ailleurs 
l'admet lui-même, comme le montre très bien Jean-Claude Kaufmann, et comme le rappelle historiquement Francesca Cancian. En envisageant ainsi le romantisme, l'idéologie fissionnelle ignore ou dissimule le pouvoir qui y est à l'œuvre, en simplifiant ce qu'elle construit comme son contraire et en exaltant la figure du « tiers » qui permettrait de « "casse[r]" la structure de domination inhérente au couple ${ }^{18} \gg$, sans qu'il ne soit jamais précisé par quelles autres inégalités cette structure de domination est remplacée, étant entendu qu'une relation purement égalitaire est impossible. L'idéal romantique est remplacé par l'idéal fissionnel qui fait l'exploit de n'être ni normatif ni moraliste mais qui entretient l'illusion qu'une fois débarrassée de la fusion et de l'inclusion, la relation amoureuse pourra dépasser la jalousie, la possession, l'oblativité et l'aliénation pour s'épanouir pleinement et librement dans l'altérité, sans jeux de pouvoir.

\section{Individualisation, communication et socialisation : Le casse-tête amoureux}

En France, les études empiriques et compréhensives de Jean-Claude Kaufmann et de François de Singly ont éclairé le rôle de l'amour dans la socialisation. L'amour extrait l'individu du soi « pour mettre en mouvement vers, attacher $\grave{a}^{19} \gg$. Moteur de l'individualisme réflexif pour les uns, source du lien social pour les autres : dans les visées sociologiques, l'amour est sans cesse tiraillé entre l'individu et le collectif, engendrant de fortes dissonances cognitives. Comment allier des indépendances si durement acquises et des identités si longuement travaillées ? Comment peuvent-elles faire lien sans se trahir, ladite trahison reposant à la fois dans le sacrifice personnel mais aussi dans le refus de toute évolution identitaire ? Comme le souligne bien Kaufmann, « le défi amoureux désormais est de rattacher d'une manière différente l'individu après qu'il a été séparé ${ }^{20} \gg:$ l'amour est à réinventer dans notre modernité avancée. Pour cela, les anciens outils grecs comme l'agapè et la philia peuvent être précieux. L'« agapè conjugale », selon l'expression de Kaufmann, décrit cette réinvention de l'amour après des premiers temps plus passionnels, cette capacité à construire à deux un être-bien. Cet agapè est sans cesse menacé par l'idéal de l'individu rationnel hérité des Lumières. L'apaisement qu'il apporte a aussi été souligné par Luc Boltanski pour qui ce régime d'amour permet aux individus 
de se protéger mutuellement à tour de rôle, d'embrasser leurs faiblesses, et d'atteindre une égalité (pas forcément matérielle) des dons et contre-dons. L'agapè comme régime d'action accueille pleinement la réflexivité contemporaine et l'amour de soi comme « point d'appui [pour] discerner le besoin de celui que l'on croise et d'aller à son devant ${ }^{21} \gg$, point d'appui donc de la générosité et de la bienveillance. Dans la même mouvance, François de Singly plaide pour l'abandon de l'équivalence et pour une redéfinition de l'amour qui « conserve bien des qualités de l'agapè tout en respectant les exigences de la philia [pour construire un] espace où savoir compter au sens ordinaire du terme n'interdit ni de compter sur l'autre, ni d'agir pour que le partenaire puisse compter sur $\operatorname{soi}^{22} \gg$. Une telle réinvention doit inclure les mutations identitaires propres aux individus, y compris celles apportées par les aventures amoureuses. Plus largement, Kaufmann et de Singly ont permis de comprendre les influences mutuelles de la construction individuelle et des modèles amoureux, privilégient l'étude des routines, des espoirs et des rêves. Les amours sont traversées des imaginaires sociaux et imprégnées conjointement des rêves et des sacrifices personnels dont Kaufmann a bien montré qu'ils ne sont pas réductibles à une supposée aliénation (souvent du féminin) dans et par le couple.

\section{Du romantisme à la relation pure ?}

Anthony Giddens postule lui aussi une métamorphose cruciale de l'amour, qu'il définit comme un « mode d'organisation de la vie personnelle étroitement lié à la colonisation de l'avenir et à la construction de l'identité personnelle ${ }^{23} \gg$. L'individualisme a remis en cause l'amour romantique mais s'est échoué sur les promesses du consumérisme individuel. Un modèle amoureux se porte à son chevet : la relation pure. C'est une « relation de stricte égalité sexuelle et émotionnelle, porteuse de connotations explosives vis-à-vis des formes préexistantes du pouvoir tel qu'il était traditionnellement réparti entre les deux $\operatorname{sexes}^{24} \gg$ et qui comporte deux éléments principaux et interdépendants : d'abord un amour convergent, « actif, contingent », qui s'oppose au « seul et unique », au « pour toujours »du romantisme, et qui « ne peut se développer que dans une société où pratiquement chacun a une chance de parvenir à son propre accomplissement sexuel $^{25} \gg$. La convergence a pour effet, et non pour cause, des séparations 
de plus en plus fréquentes et de plus en plus prévisibles, ce que retranscrit la capacité à priori paradoxale qu'ont les individus à entrer en relation amoureuse avec quelqu'un tout en sachant que cela ne durera probablement pas toute la vie. Au fond, explique Giddens, c'est moins un partenaire idéal qu'une relation parfaite qui est recherchée, parfaite en ce qu'elle respecte le lourd et réflexif travail identitaire effectué jusqu' ici par l'individu. Or, s'il respecte les individualités respectives, l'amour convergent est donc caractérisé par « l'égalité la plus stricte en termes de donation et de réception émotionnelles ${ }^{26} \gg$. L'égalité n'est pas celle de la répartition de l'oblativité entre hommes et femmes, dont on sait qu'elle est aujourd'hui une charge féminine. Une telle répartition, aussi équitable soit-elle, serait une continuation du régime romantique, qui alimente et qui est alimenté par le don tout entier de ses amoureux. Au contraire, l'égalité ici décrite n'envisage plus l' individu comme étant le fleuve affluent du couple et ambitionne donc de supprimer l'oblativité qui entrave le bon développement de l'individualité. La relation pure repose ainsi sur la suppression de cette illusoire totalité romantique pour privilégier les constructions du moi et $\mathrm{du}$ couple qui s'influencent sans se fondre l'une dans l'autre. Il faut ici revenir à des questions en apparence simples mais pourtant fondamentales. Qu'est-ce qu'un couple ? Fait consensus la réponse selon laquelle il est une rencontre entre individus qui crée un autre monde, mais elle porte en elle une autre question : cette totalité s'autoalimente-t-elle ou se nourrit-elle d'un abandon et d'un déni des qualités des parties qui la composent ? Pour le romantisme, le couple doit noyer les individualités. Pour la relation pure, il est, au contraire, composé par elles. C'est pourquoi ce nouveau mode amoureux suppose le minutieux travail d'une démocratie relationnelle qui prend en charge les éléments les plus quotidiens et les plus triviaux comme les plus structurants et les plus emblématiques des individus dont on réalise qu'ils sont les éléments décisifs du couple. Il devient bien sûr nécessaire pour l'individu évolutif de disposer d'un espace de liberté suffisamment large pour se construire solidement et pour maintenir une certaine constance à travers les événements. Cela permet, au sein du couple, de communiquer ses envies et ses attentes, non pas pour exercer la tyrannie d'un moi égoïste et égocentrique mais, comme dans toute démocratie, pour négocier la relation et établir des compromis qui permettent au régime de se poursuivre. L'individualisme doit aussi comporter l'aptitude à se rendre vulnérable en 
s'ouvrant à l'autre, pour mieux prendre en charge les politiques identitaires et amoureuses : « la connaissance des caractéristiques les plus intimes d'autrui remplit une fonction absolument centrale ${ }^{27} \gg$, ce que des auteurs critiques ont pu qualifier de tyrannie communicationnelle.

En plus de cet amour convergent, une deuxième caractéristique est nécessaire : une sexualité plastique, « décentrée, affranchie des exigences de la reproduction ${ }^{28}$ » qui, à l'inverse de l'amour romantique, mobilise un ars erotica. Du fait de la libération sexuelle (en cela, Giddens réintègre une rupture que Foucault avait balayée), les corps se libèrent et le plaisir sexuel devient un ingrédient clé d'une relation amoureuse, le « côté-face » de l'amour convergent. L'émancipation des femmes passe aussi par l'émancipation de leurs corps trop souvent définis d'une part par une fonction reproductrice, d'autre part par une dichotomie mère/putain, l'ensemble assignant tout droit à la domesticité. Mais la libération sexuelle est explosive. Du féminin, elle exprime les désirs jusqu'ici garrottés, déploie et découvre les corps qui alors expulsent la fonction maternelle, revendique la liberté de ne pas être mère et d'être putain. La sexualité devient un territoire personnel et social dans lequel se joue la liberté fondamentale à disposer de son corps. De ce fait, les comportements sexuels libérés ne sont pas, comme les qualifie avec mépris Eva Illouz, une pâle imitation des comportements masculins ni l'expression détournée d'une domination masculine qui « écrit les règles de la reconnaissance et de l'engagement ${ }^{29}$ », mais ils font en revanche bien partie d'un mouvement plus large quant à la réappropriation des corps confisqués, une égalité de fait inséparable à cet égard des égalités de droit comme la légalisation de l'avortement.

Il faut souligner avec force que la ligne défendue par Anthony Giddens n'est pas un détachement de l'individu hédoniste et narcissique menant tout droit à l'ostracisme, mais plutôt un détachement vis-à-vis de l'idée qu'il faudrait, pour être complet, avoir un partenaire qui devrait de surcroît répondre à des normes romantiques. La critique porte sur « l'identification projective $^{30} \gg$, cette fusion amoureuse qui suppose une complémentarité de la différence sexuelle, laquelle encourage, au sein d'une commune subordination à la norme qu'est le couple, la continuation de la domination masculine. Si elle a pu aider à une émancipation de la tradition, la validation de l'identité individuelle par un unique autre telle que l'entend le complexe de l'amour romantique est aujourd'hui un vrai problème dans une modernité faite de 
liens plus faibles mais multipliés et électifs, une modernité également sensible aux égalités de genre. Toutefois, François de Singly a raison de souligner que la fusion est toujours envisagée chez Giddens comme une « invasion possible », oubliant par là « la possibilité de créer, de maintenir une relation grâce à un renoncement volontaire d'un de ses territoires personnels ${ }^{31}{ }$. Mais Giddens ne perçoit pas uniquement cette « invasion possible » dans la fusion, et c'est d'ailleurs certainement là la richesse de sa proposition : si la relation pure est méfiante envers la fusion romantique, c'est bien parce qu'elle est chargée de rapports de pouvoir genrés. Ainsi, le « renoncement volontaire » est le plus souvent celui du féminin. D’ailleurs, si Giddens est l'auteur qui identifie avec le plus de finesse le déploiement de la communication démocratique dans le couple, la rationalité émotionnelle qu'il postule laisse peu de place au déraisonnable et aux imaginaires dont Simmel, puis Morin ou Kaufmann, ont montré le rôle essentiel dans la construction de la réalité amoureuse. Le flou, l'approximatif, le lyrisme, les rêves n'y sont envisagés que comme des mystifications romantiques qu'il convient de chasser au plus vite. Il s'agit là d'une critique majeure qu'il faut formuler à l'égard d'Anthony Giddens qui, dans sa démocratie relationnelle presque habermasienne, laisse peu de place à l'imagination. Chat échaudé craint l'eau froide : si la relation pure est prudente à l'égard de la fusion romantique, la poésie et le renoncement, c'est parce qu'elle a compris qu'hérité du romantisme, le sacrifice est celui de l'épanouissement, souvent féminin.

Face à l'impossibilité de le redéfinir selon les critères contemporains d'égalités de genre, il semble donc nécessaire de changer de modèle. En ce sens, la tentative de François de Singly pour concilier les contraires en des propositions qu' ils nomment « individualisme relationnel » ou « modèle du double respect » est, théoriquement du moins, intéressante parce qu'elle met l'emphase sur la possibilité de s'épanouir individuellement sans renier les liens à autrui, permettant « la recherche d'un équilibre entre l'intimité personnelle et l'intimité conjugale ${ }^{32} \gg$. Le romantisme continue de soustendre les imaginaires et les pratiques, que ce soit dans les représentations culturelles et médiatiques ou dans les nouvelles formes de rencontre amoureuse sur Internet. Mais ce mouvement moderne qui promettait l'émancipation individuelle contre les assignations familiales semble être devenu ce contre quoi il s'est historiquement constitué : une structure étouffant les aspirations individuelles. Dans une modernité avancée faite de liens plus 
faibles, mais aussi plus nombreux et plus libres, la validation de l'identité individuelle par un unique autre, telle que la suppose le complexe romantique, est un vrai problème et l'horizon de nouvelles recherches théoriques et empiriques à mener.

L'intercompréhension apparaît, en théorie, comme la meilleure prévention contre la rupture, même si les codes communicationnels qui la construisent restent eux-mêmes sujets aux irréductibles tensions entre préservation de l'individu et mouvement vers l'autre. Négociations, compromis, concessions, renoncements, expression personnelle sont autant d'outils qui inciteraient à la démocratie relationnelle pour les auteurs compréhensifs ou à la tyrannie expressiviste pour les auteurs critiques. Cette divergence théorique est bien illustrée par la façon dont ces paradigmes envisagent les temporalités du couple : les auteurs compréhensifs insistent davantage sur les moments conjugaux que les auteurs critiques, qui évoquent les relations amoureuses de façon plus générale. Or, entendue comme un idéal plutôt que comme la possibilité de sonder absolument l'autre, l'intercompréhension apparaît comme une somme de moments et non comme un continuum qui ne serait en fait qu'une reformulation de la connaissance intuitive, c'est-à-dire romantique, de l'autre. 


\section{Notes}

1 Beck, Ulrich, Beck-Gernsheim, Elizabeth, The Normal Chaos of Love, Cambridge, Polity Press, 1995.

2 Beck, Ulrich, Beck-Gernsheim, Elizabeth, ibid., p. 12.

3 Beck, Ulrich, Beck-Gernsheim, Elizabeth, ibid., p. 49.

4 Beck, Ulrich, Beck-Gernsheim, Elizabeth, ibid.

5 Giddens, Anthony, La Transformation de l'intimité. Sexualité, amour, et érotisme dans les sociétés modernes, Rodez, Éditions du Rouergue, 2004 , p. 80.

6 Kaufmann, Jean-Claude, L'Étrange histoire de l'amour heureux, Paris, Fayard, 2010, p. 82.

7 Giddens, Anthony, ibid., p. 59.

8 Cancian, Francesca, Love in America. Gender and Self-Development, Cambridge University Press, 1991.

9 Ryan, Mary P., Cradle of the Middle Class. The Family in Oneida County, New York, 1790-1865, Cambridge, Cambridge University Press, 1983.

10 Cancian, Francesca, ibid., p. 5.

11 Cancian, Francesca, ibid., p. 70.

12 Cancian, Francesca, ibid., p. 77.

13 Bauman, Zygmunt, L'Amour liquide. De la fragilité des liens entre les hommes, Paris, Fayard, 2010.

14 Chaumier, Serge, L'Amour fissionnel. Le nouvel art d'aimer, Paris, Fayard, 2004 ; Chaumier, Serge, La Déliaison amoureuse. De la fusion romantique au désir d'indépendance, Paris, Payot, 2004.

15 Beck, Ulrich, Beck-Gernsheim, Elizabeth, ibid.

16 Giddens, Anthony, ibid.

17 Chaumier, Serge, La Déliaison amoureuse..., ibid., p. 298.

18 Chaumier, Serge, L'Amour fissionnel..., ibid., p. 134.

19 Kaufmann, Jean-Claude, ibid., p. 13.

20 Kaufmann, Jean-Claude, ibid., p. 66.

21 Boltanski, Luc, L'Amour et la Justice comme compétences, Paris, Gallimard, 1990, p. 263.

22 Singly (de), François, Séparée. Vivre l'expérience de la rupture, Paris, Armand Colin, 2011, p. 223. 
23 Giddens, Anthony, ibid., p. 78.

24 Giddens, Anthony, ibid., p. 10.

25 Giddens, Anthony, ibid., p. 82.

26 Giddens, Anthony, ibid., p. 81.

27 Giddens, Anthony, ibid., p. 83.

28 Giddens, Anthony, ibid., p. 10.

29 Illouz, Eva, Why Love Hurts. A Sociological Explanation, Cambridge, Polity Press, 2012, p. 137.

30 Giddens, Anthony, ibid.

31 Singly (de), François, ibid.

32 Singly (de), François, ibid. 


\section{Femmes et médias: courants de recherche}

\section{Une première étape dénonciatrice}

Les premières approches sur les représentations des femmes dans les médias ont relevé de critiques dénonciatrices, largement fonctionnalistes, dans le cadre de théories féministes. Considérant que les représentations ont le pouvoir de diffuser le sexisme dans les imaginaires sociaux, de manière peut-être encore plus sournoise que des discours publics dont on suppose qu' ils recourent moins à l'identification ou à l'affectif, cette première vague définit les médias de masse comme des agents de contrôle du monde social. Un article typique de cette mouvance est par exemple celui de la marxiste Lillian Robinson, « What's My Line? Telefiction and Women's Work » dans lequel, sans tomber néanmoins dans le piège de la fascination des publics, l'auteure suggère que « le colonialisme de la culture de masse, l'impérialisme domestique de la télévision, rencontre de la résistance [et est] l'un des facteurs qui influencent la conscience des femmes ${ }^{1} \gg$.

La première pierre est posée en 1963 par la fondatrice de la National Organisation for Women (NOW), Betty Friedan, dans La Femme mystifiée ${ }^{2}$. Dans cet ouvrage, Friedan critique la «mystique » propagée à partir des années 1950 par la presse magazine dans laquelle elle a elle-même longtemps travaillé. À l'inverse des représentations des années 1930, cette presse exalte la figure de la mère au foyer, montrant en négatif la misère et le désarroi des femmes actives. Dans un autre ouvrage canonique, La Femme eunuque ${ }^{3}$, publié en 1971, Germaine Greer fustige les idéaux romantiques des médias de masse, les accusant de faire croire aux femmes que la relation amoureuse hétérosexuelle est la clé de leur épanouissement personnel. Il faut souligner 
qu'en France, Evelyne Sullerot (elle aussi très impliquée dans les mouvements militants puisque co-fondatrice, en 1956, du Planning familial) produit, en 1964, à partir d'un corpus de presse féminine, une étude similaire mais aux résultats plus nuancés ${ }^{4}$. Répertoriant les figures dominantes de femmes dans la presse (la « dame », la « femme », la « ménagère »), elle diagnostique un syncrétisme médiatique mêlant optimisme et angoisse. À rebours des travaux nord-américains qui dénoncent les médias et leurs monstrations, la perspective adoptée par Sullerot et les résultats qui en découlent sont annonciateurs d'une critique plus compréhensive.

Lorsque les premières féministes prennent les médias pour objet d'étude, c'est pour en critiquer les stéréotypes à l'œuvre, considérant que les industries culturelles sont contrôlées par le patriarcat. Les liens qui sont tissés entre études féministes et études communicationnelles relèvent davantage de l'emprunt que de l'articulation : les études féministes s'aident d'outils comme l'analyse de contenu alors privilégiée par les chercheurs en communication, mais n'abandonnent pas leur épistémologie marxiste ni ne considèrent les possibles réappropriations de ces messages. De leur côté, les études communicationnelles utilisent l'approche du genre élaborée par les théories féministes pour mesurer les écarts entre la réalité et sa représentation médiatique. Les travaux de George Gerbner en sont significatifs. S'extrayant du paradigme des effets directs sans renier l'influence des médias sur les représentations collectives, ses recherches ont apporté des éléments intéressants sur la différenciation des « rôles sexués » (sex roles), éclairant la façon dont les médias se substituent à la parole des femmes en ne les plaçant que rarement comme premières locutrices ${ }^{5}$. La vue d'ensemble que permet l'analyse de contenu a donné des résultats fondateurs sur la sous-représentation et la surdétermination des profils féminins. Dans leur étude des séries dramatiques diffusées sur les networks entre 1969 et 1979², George Gerbner et Nancy Signorielli notent ainsi un grand déséquilibre entre hommes et femmes $(74,4 \%$ des 4330 personnages recensés sont des hommes), mais un engagement plus fort des femmes envers la famille et envers la conjugalité puisque la moitié d'entre elles vivent une relation amoureuse, contre seulement un tiers des hommes. De plus, les personnages féminins qui travaillent le font dans des milieux traditionnellement codés féminins (elles sont infirmières, secrétaires ou serveuses), et ont en contrepartie une vie familiale ou amoureuse insatisfaisante voire quasi nulle. 
On sait traditionnellement que l'analyse de contenu, en plus de porter en elle les biais du chercheur qui rend la représentation univoque en la catégorisant, n'intègre pas de réflexions poussées sur les rapports de sens qui y sont à l'œuvre. La caractérisation d'un personnage, par exemple, est largement influencée par les questions que pose le chercheur à son égard et elle ne rend pas vraiment compte des relations avec les autres personnages, de sorte que la distribution qualitative du pouvoir entre hommes et femmes échappe à l'analyse quantitative. Cette critique de la « distorsion ${ }^{7} »$, selon l'expression de Liesbet van Zoonen, entre les représentations et la réalité relève d'une approche réflective qui considère que les médias de masse sont un miroir tendu à la société, et dont Stuart Hall a bien montré les écueils : d'une part, comment produire une définition stable de la représentation avec laquelle comparer ce qui doit également être une définition stable de la réalité ? D’autre part, quels critères utiliser pour mesurer cet écart ? La fronde menée par les féministes contre les contenus médiatiques souffre d'une épistémologie inadaptée à l'étude des imaginaires médiatiques - en témoignent les démarches largement dénonciatrices et prescriptives ou le flou théorique autour du concept de stéréotype ${ }^{8}$. Mais elle a eu pour bénéfices d'alerter sur la nécessité de produire d'autres représentations des femmes ainsi que d'amorcer l'articulation entre combats militants, relatifs aux conditions matérielles d'existence des femmes, et combats symboliques. Ces nouvelles luttes ont porté en premier lieu contre les représentations jugées uniformément sexistes, puis dans les représentations, alors entendues comme des territoires où s'expriment des luttes de définition du monde, c'est-à-dire comme relais ou producteurs d'idéologies.

Si ce que Gaye Tuchman appelle l'« annihilation symbolique' » des femmes a été la préoccupation majeure des premières études sur les rôles types proposés par les médias, elle a été suivie d'une construction épistémologique plus complexe, se détachant du paradigme fonctionnaliste. En son sein, le concept de représentation devient rapidement et durablement, comme le résume bien Liesbet van Zoonen dans son ouvrage de référence Feminist Media Studies en 1994, le « champ de bataille crucial pour le féminisme contemporain ${ }^{10} \gg$. Le découpage et la cartographie des courants féministes au croisement de l'analyse des médias et des études féministes varient bien entendu selon les sources scientifiques, certaines dessinant les correspondances entre les intérêts principaux des courants féministes et 
leurs manières d'envisager les médias de masse ${ }^{11}$, quand d'autres insistent sur la multiplication et la complexification des variables (de production et de réception, ou de polysémie du texte) intégrées dans les analyses des médias ${ }^{12}$. Retraçant l'histoire des feminist media studies, Michèle Mattelart rappelle que l'on ne peut dissocier leur construction d'un ensemble de mutations socioculturelles et politiques, comme la « crise des utopies sociales, [la] crise des identités et grands récits d'émancipation, [la] crise des modèles de légitimation des savoirs et des actions ${ }^{13} \gg$, des crises qui ont elles-mêmes été animées par les idéaux féministes. Les « carences » françaises dans ces domaines proviennent selon elle de la difficulté de dépasser l'opposition entre culture et industrie, de considérer les médias « féminins » comme sérieux et de construire, en conséquence, des formes institutionnelles sur le sujet (UFR, département, laboratoire de recherche). De son côté, Marlène Coulomb-Gully souligne les résistances des sciences de l'information et de la communication à l'égard des gender studies. Les women's studies sont issues du féminisme de la deuxième vague, ce qui a été un frein à leur intégration dans l'université, tant du côté des féministes elles-mêmes (refusant d'être « vendues » à l'institution ou « récupérées » par elle) que de l'université (considérant comme académiquement illégitime le domaine des inégalités de genre). Les études de genre se développent, en France, dans les années 1980-1990, en partant de l'histoire et de la sociologie vers la philosophie et la science politique ${ }^{14}$. Dressant le bilan des publications scientifiques, Coulomb-Gully situe ainsi un «gender turn » au début des années 2000, à partir duquel les recherches en communication ignorent de moins en moins celles sur le genre.

Les différents courants du féminisme (libéral, radical, matérialiste, socialiste, etc.) ont pesé sur les méthodes et les paradigmes d'analyse des médias. L'idée, par exemple, que les médias de masse sont le canal par lequel les hommes, qui en commandent largement la production, reproduisent la domination des femmes par le biais de stéréotypes, a été largement critiquée par Noreene Janus comme relevant du féminisme libéral. Pour cette dernière, lorsque les auteurs des analyses de contenu déplorent que les femmes n'aient pas accès aux mêmes représentations que les hommes, ils plaident en réalité pour l'«intégration des femmes dans le système actuel, sur la même base que les hommes ${ }^{15} \gg$, mais sans le subvertir. De même, est sous-estimée la diversité des modes de production 
médiatique et des structures de l'organisation politique. Janus, en retour, défend une posture historique et holistique qui articule les représentations avec le contexte social dans lequel elles sont produites, non pas en tant que reflet, mais comme le jeu d'une relation complexe et dialectique entre économie médiatique, place des femmes et contenu médiatique. Les représentations médiatiques se sont peu à peu «émancipées » des paradigmes féministes qui les considéraient comme un miroir déformant ou une tribune contrôlée par le patriarcat pour investir une place culturelle et symbolique dans la sphère publique.

\section{Le bovillonnement épistémologique des film studies: sémiotique, psychanalyse et approches structurales de l'idéologie}

D’un autre côté, est visible un bouillonnement épistémologique dans les film studies, qui mélangent sémiotique, psychanalyse et approches structurales de l'idéologie. Les études filmiques féministes (feminist film studies), inspirées des women's studies qu'elles ont en retour influencées, sont originellement scindées en deux grands courants. Le premier, étasunien, se présente comme une sociologie du cinéma qui analyse la description et la légitimation des productions et des réceptions féminines, et dont la revue Women \& Film est la première tribune. Le second, britannique, offre une méthodologie qui se dit plus objective. Mobilisant les outils de la sémiologie et de la psychanalyse, « cette approche a essayé de comprendre comment les films signifient - elle a essayé d'aller plus loin que la vision de l'image pour en analyser sa structure, ses codes, le sous-texte général du film ${ }^{16} \gg$. Combinaison théorique de sémiotique, de marxisme althussérien et de psychanalyse, les objets des études filmiques féministes recouvrent les productions de subjectivités et d'identités par les représentations, les pratiques spectatorielles comme les processus d'identification ou encore les articulations entre désir, fantasme et représentations. Elles cherchent notamment à cartographier les places qu'occupent hommes et femmes dans les représentations cinématographiques : les premiers tendent à être des sujets avec lesquels s'identifient les agents de la production tandis que les secondes sont objectivées par le plaisir masculin via un processus de fétichisation. B. Ruby Rich prend pour archétypes de ces travaux ceux de Claire Johnston 
et de Laura Mulvey, dont les articles « Women's Cinema as CounterCinema » (1973) et «Visual Pleasure and Narrative Cinema » (1975) sont souvent cités comme les textes fondateurs de la théorie filmique féministe. Toutes deux contribuent à la compréhension des représentations des femmes et à la construction du plaisir - plaisir cinématographique et plaisir sexuel - dans une analyse largement influencée par la psychanalyse : les premières contributions des théories filmiques féministes entendent comprendre la « construction de la position des sujets dans l'idéologie, tandis que les narrations hautement œdipiennes les prêtaient à une lecture des mécanismes inconscients de différence sexuelle dans notre culture ${ }^{17} \gg$. Pour Johnston et Mulvey, les femmes sont absentes du processus de représentation, pour deux raisons. Tout d'abord, parce qu'elles « ne se représente[nt] pas [elles-mêmes] mais, par un processus de déplacement, représente[nt] le phallus masculin » : autrement dit, thème classique de la psychanalyse, les femmes représentent l'absence de phallus ${ }^{18}$. L'une des subversions possibles de ce plaisir phallocentré, plaide Johnston, se loge dans la réappropriation de la dimension divertissante du cinéma par les femmes. L'auteure déplore la dénonciation des médias par les féministes, dénonciation qui provient selon elle d'une longue tradition de critique bourgeoise et qui rend stérile toute discussion scientifique sur les représentations des femmes dans le cinéma ou sur les productions féminines cinématographiques. La seconde raison pour laquelle les femmes sont rayées des représentations est que le tout-puissant regard masculin (male gaze) objective, selon Mulvey, le corps féminin et rend impossible l'expression d'une vision féminine ${ }^{19}$. Les personnages féminins tout comme les réceptrices sont privés de l'expression d'un plaisir féminin, pris au piège du male gaze. Cette vision de la subjectivité féminine est jugée appauvrissante et essentialiste deux décennies plus tard par bell hooks ${ }^{20}$, qui défend la possibilité d'un « regard oppositionnel » (oppositional gaze). De son côté, Barbara Creed montre que la distribution binaire entre un homme actif et une femme passive n'est pas anhistorique : le cinéma a une longue histoire de représentations d'une « monstruosité féminine » qui engage la fonction reproductrice et maternelle ${ }^{21}$.

À la fin des années 1970, les théories filmiques féministes enrichissent leurs travaux d'une critique des outils psychanalytiques, souvent trop limités pour analyser les nombreuses subjectivités à l'œuvre dans les représentations et dans les réceptions. Comme le souligne Geneviève Sellier, 
le cinéma est « bien plus divers dans la représentation des femmes et des rapports sociaux de sexe, à la fois dans les films et par leur contexte de production et de réception ${ }^{22} \gg$. Les écrits de Linda Williams ou de Teresa de Lauretis marquent également cette nouvelle étape, en formulant de nouvelles questions sur les représentations des femmes dans les médias du fait de leurs «multiples perspectives sur le soi, la capacité d'agir, l'identité et l'environnement culturel du sujet ${ }^{23} \gg$. Les recherches de Teresa de Lauretis sur Rebecca, le premier film d'Hitchcock, vont plus loin que les propositions, déjà très novatrices, de Tania Modleski concernant une « possibilité (limitée) d'un désir spécifiquement féminin ${ }^{24}$ ». De Lauretis ambitionne d'extraire les femmes du piège de l'objectivation et du plaisir patriarcal en théorisant un désir féminin double, à la fois actif et passif, situé « dans le désir de l'autre [mais aussi] dans le désir d'être désirée par l'autre ${ }^{25} \gg$. La rencontre, au début des années 1990, des film studies et des cultural studies fait émerger des travaux sur les liens entre les représentations cinématographiques et le contexte socioculturel dans lequel elles sont produites. Geneviève Sellier cite les travaux fondateurs d'Edward B. Turk à propos des influences de l'homosexualité de Carné sur son cinéma ${ }^{26}$, ou ceux de Jackie Stacey sur les relations entre les modalités de représentation des stars, les pratiques des spectatrices et la construction de leurs propres identités, entre 1940 et $1950^{27}$. L'approche des cultural studies, en interrogeant les axiologies, a permis d'ouvrir les film studies à d'autres approches et d'autres objets, notamment la télévision.

\section{La consubtantialité des hiérarchies culturelles et des hiérarchies de genre}

Ces glissements ont été permis par une critique de l'articulation des dichotomies hiérarchisées noble/populaire et masculin/féminin, syllogisme menant à la valorisation des cultures codées « masculines » et au mépris de celles jugées « féminines » (comme le mélodrame). La consubstantialité des hiérarchies culturelles et des hiérarchies de genre est un vrai problème dans l'étude des médiacultures, notamment sentimentales. Dans leur état des lieux des études cinématographiques, Brigitte Rollet et Geneviève Sellier soulignent la réticence de certaines auteures françaises à mettre en avant leur genre pour contextualiser ou analyser leurs 
œuvres et l'expliquent ainsi : « la constitution [des études filmiques et des études culturelles] dans les années 1960 et 1970 en Grande-Bretagne et aux États-Unis s'est faite dans un contexte politique de contestation du savoir académique, à partir d'une critique du pouvoir patriarcal et de l'élitisme culturel qui ne connaît pas d'écho en France ${ }^{28} \gg$. Or, prendre conscience que les médias eux-mêmes sont ancrés dans des contextes sociaux, dans des systèmes de genre notamment, et sont donc genrés jusque dans leur axiologie éclaire d'un jour nouveau les différentes réactions que suscite la télévision. Les hiérarchies culturelles ne se situent pas par la grâce de l'objectivité culturelle et artistique au-dessus des idéologies du genre mais sont au contraire en articulation avec elles. Lynn Spigel a d'ailleurs bien montré que l'émergence d'une culture féminine participe de la suspicion de toute une presse à l'égard de la télévision que l'on accuse de défier l'autorité paternelle ${ }^{29}$. La détestation globale des médias de masse (qui se transforme en franche aversion dès qu'il s'agit des soap operas, registre féminin d'un média féminin) se confond avec une détestation du féminin pour Andreas Huyssen ainsi que pour Geneviève Sellier et Noël Burch : depuis l'avènement du modernisme ${ }^{30}$, « les critiques culturels ont souvent exprimé leur dédain pour les médias de masse dans un langage qui évoque le mépris pour les qualités que le patriarcat assigne au féminin ${ }^{31} \gg$. Sont honnies l'émotion, la passivité, la subjectivité, l'infériorité en même temps que les hommes émergent comme des auteurs de génie, des individus objectifs dans une littérature authentique, capables d'ironie et contrôlant parfaitement les moyens esthétiques, comme dans l'emblématique roman du modernisme Madame Bovary ${ }^{32}$. Comme le résume bien Noël Burch dans son formidable ouvrage De la beauté des latrines : « le créateur moderniste a toujours eu besoin de la culture de masse comme Autre, comme étalon "féminisé” de cette supériorité "masculine" qui découle de la rationalité de ses formes, de la conscience qu'il a de sa propre écriture, de la distance (《 clinique ») qu'il observe face à l'épanchement vulgaire des sentiments (le mélo est bien l'Autre irréductible de l'art légitime $)^{33} \gg$. Le discours « politique, psychologique et esthétique ${ }^{34} \gg$ du début $\mathrm{du} \mathrm{xx}^{\mathrm{e}}$ siècle distribue de façon genrée la culture de masse aux femmes et la culture noble aux hommes. En retour, Geneviève Sellier a bien montré que des formes «cultivées » comme la Nouvelle Vague, qui peuvent être innovantes sur le plan formel, sont des lieux d'expression masculine, qui prennent paradoxalement peu en 
charge les controverses sociales et qui, plutôt que d'explorer les « nouveaux rapports entre hommes et femmes », soumettent les personnages féminins à des « constructions fantasmatiques masculines ${ }^{35}$ ». Pour construire un champ d'analyse de la télévision et des femmes, il a donc fallu entreprendre une légitimation de ces recherches féministes de la télévision, transperçant les à priori d'un contenu creux et d'une réception passive. Ainsi que le résume Bruno Péquignot à propos des romans sentimentaux longtemps abhorrés, « l'intérêt sociologique de l'analyse des romans sentimentaux ne peut être appréhendé qu'à cesser de mépriser cet objet et par-delà ses consommatrices $^{36} \gg$ : à la jonction du féminin et de la masse, ces détestations ne sont qu'une « forme déguisée du mépris de classe pour la "bêtise" de ces femmes du "peuple" ${ }^{37} \gg$. Critique des monstrations d'une part, élargissement du politique au privé et identifications des leviers délégitimant une analyse sérieuse des femmes et de la télévision d'autre part : en s'intéressant tout particulièrement aux identités féminines, les travaux sur la télévision et les femmes ont pris pour socle les enseignements de la deuxième vague féministe.

Les études sur les soap operas ont fortement participé de la revalorisation des productions dites féminines. Ce genre intéresse particulièrement les études télévisuelles durant les années 1970 et jusqu’au début des années $1980^{38}$ du fait de son caractère codé féminin, de son traitement politique des affaires privées, de son usage de la métaphore et de la nécessité d'adopter à l'égard de la consommation jugée ridicule des femmes une démarche compréhensive ${ }^{39}$. De telles analyses éclairent l'ouverture sur le monde que favorisent les soap operas pour des femmes souvent confinées au foyer familial. Parmi elles, et contre le psychologisme de Carol Lopate pour qui ces programmes « remplissaient les longues journées des femmes en dirigeant leurs fantasmes vers l'amour et la famille, promettant que leur vie est tout ce dont elles ont besoin ${ }^{40} \gg$, Tania Modleski souligne le décalage entre le style de vie nucléaire desdites femmes au foyer et celui des familles très étendues des soap operas ${ }^{41}$, qui démontre tout l'attrait du féminin pour une collectivité portée sur les conversations, les longs échanges et les émotions ${ }^{42}$. Dans la même veine, Charlotte Brunsdon postule que l'intérêt des spectatrices pour les soap operas provient de leurs récits largement relationnels, structurés autour d'interminables négociations orales concernant la sphère personnelle et familiale. Par ailleurs, Christine Geraghty identifie les tensions féministes 
qui y sont à l'œuvre, montrant que les soap operas critiquent les patriarches tout en réaffirmant l'importance des liens familiaux ${ }^{43}$. Ces découvertes sont faites également dans le domaine de la littérature sentimentale, comme l'ont montré les recherches canoniques de Janice Radway ${ }^{44}$. Là encore, ces divers travaux ont encouragé le développement d'une démarche plus compréhensive à l'égard des représentations et des réceptions des médias de masse, loin des dérives prescriptives, normatives ou moralistes.

Aujourd'hui, le courant de recherche le plus actif sur le thème des femmes et de la télévision reste celui de la « critique féministe de la télévision » (feminist television criticism $^{45}$ ), rattaché parfois prudemment aux cultural studies, dont se réclament des auteures comme Charlotte Brunsdon, Julie d'Acci, Lynn Spigel, Kim Akass ou Janet McCabe, et auxquelles on peut adjoindre Bonnie Dow et Angela McRobbie. Trois paradigmes y primeraient, selon Amanda Lotz ${ }^{46}$, qui prend régulièrement ses distances avec ce mouvement en raison de ses dimensions parfois encore très critiques : le premier paradigme met l'accent sur les héroïnes pour construire une typologie ou des stéréotypies, à l'instar de la « nouvelle femme » ou de la «femme indisciplinée ${ }^{47} \gg ;$ le deuxième envisage les contenus féministes comme des stratégies narratives essayant de développer ou de contenir des sujets féministes (avec par exemple Judith Mayne ${ }^{48}$ ou Danae $\mathrm{Clark}^{49}$ ) ; enfin le troisième paradigme, dans lequel s'inscrivent notamment les recherches postféministes, inventorie les déploiements discursifs des représentations féministes - l'ouvrage de Bonnie Dow, Prime Time Feminism, faisant ici autorité. Pour la feminist television criticism, est nécessaire une redéfinition du politique qui ne le contient plus aux affaires publiques, juridiques et économiques mais qui accepte les problématiques privées de genre, de sexualité ou de domesticité ainsi que la question des imaginaires culturels. À la télévision aussi, le personnel est politique.

\section{Au-delà du fonctionnalisme et du structuralisme, vers le conflit de sens}

Ce basculement d'une approche réflective vers une approche constructiviste opère grâce au concept d'idéologie, pour lequel le féminisme radical, et plus précisément le féminisme socialiste ${ }^{50}$, ont eu un regain d'intérêt. 
S'inspirant des épistémologies marxistes et néo-marxistes, les études féministes sur les médias considèrent l'idéologie comme le concept clé pour comprendre comment les rapports sociaux sont maintenus et reproduits. Par cette opération, c'est tout le paradigme de la domination qui est mis à mal car les rapports de pouvoir n'y sont plus envisagés de façon mécaniquement et brutalement descendante comme dans le schéma classique de la domination (où les dominants unifiés exercent l'oppression sur les dominés démunis), mais, grâce au tournant gramscien, désormais perçus comme des jeux complexes d'hégémonies et de contre-hégémonies, en incessante adaptation et reconstruction. D'une situation de domination, on passe à un processus hégémonique : le «marxisme [est] sans garanties », selon la formule de Stuart Hall ${ }^{51}$. Dans L'Après-patriarcat ${ }^{52}$, Éric Macé propose, lui, de ramener la domination au ressenti qu'en ont les acteurs, ressenti, peut-on ajouter, qui est en tension entre la légitimité et la subjectivité des émotions.

L'apparition du concept d'idéologie est un premier pas vers la compréhension des représentations médiatiques comme participant de la sphère publique, mais ses premières applications restent très critiques, comme le signale Liesbet van Zoonen. Le tableau récapitulatif qu'elle propose montre bien que les différentes perspectives sur les médias de masse (études des stéréotypes, de la pornographie ou de l'idéologie) partagent une définition fonctionnaliste de la communication puisque, pour autant qu'elles divergent sur les composants de la communication, elles s'accordent sur la linéarité du processus émetteur-message-récepteur.

\begin{tabular}{|l|l|l|l|l|c|}
\hline & Émetteur & Processus & Message & Processus & Effet \\
\hline Stéréotypes & Hommes & Distorsion & Stéréotype & Socialisation & Sexisme \\
\hline Pornographie & Patriarcat & Distorsion & Pornographie & Imitation & Oppression \\
\hline Idéologie & Capitalisme & Distorsion & Hégémonie & Familiarisation & Sens commun \\
\hline
\end{tabular}

Les modèles de communication dans la théorie féministe des médias, Van Zoonen, Liesbet, Feminist Media Studies, Londres, Sage Publications, 1994. 
Or, l'attention aux récepteurs et réceptrices a permis de complexifier ce schéma de communication encore très marxiste. Apparaît alors une « possible distance entre le modèle "femme objet" mis au jour par l'analyse structurale et la manière dont il est perçu et reçu dans le vécu des femmes en lutte ${ }^{53} \gg$. Du côté de l'étude de la production des représentations, les propositions abondent également : les textes sont polysémiques, chargés de niveaux d'interprétation ${ }^{54}$ qui peuvent être contradictoires, porteurs dès leur encodage de luttes internes. On peut rappeler que pour Edgar Morin, déjà, leur ambivalence et leur syncrétisme s'expliquent par les tensions industrielles et démocratiques entre innovation et standardisation, entre la nécessité de surprendre et celle de plaire au plus grand nombre ${ }^{55}$. Le modèle linéaire de la communication, à l'instar du modèle classique de la domination, doit gagner en dynamique pour mieux retranscrire l'instabilité du social et les négociations incessantes entre les acteurs. Les producteurs, les messages et les récepteurs ne sont pas homogènes, et les réactions de ces derniers ne peuvent plus être envisagées comme de simples rétroactions forcément dominées par les émetteurs.

Il est significatif que plusieurs auteures féministes elles-mêmes atténuent leurs analyses critiques lors de la « découverte de l'idéologie ${ }^{56} \gg$. Angela McRobbie publie en 1978 une étude de l'approche des médias de masse comme signifiants idéologiques ${ }^{57}$. Analysant sémiologiquement le magazine pour adolescentes Jackie, elle souligne les fortes connotations qui y sont à l'œuvre, relatives à la beauté, la domesticité et les amours. McRobbie y voit la reconduction d'une idéologie sexiste dont les effets, pour autant qu'ils peuvent rencontrer des résistances, restent très puissants. Quelques années plus tard, elle fait amende honorable et juge de cette conclusion qu'elle laisse très peu de place à une quelconque activité des publics. De la même manière, quelques années après la publication de «Visual Pleasure and Narrative Cinema ${ }^{58} \gg$, Laura Mulvey elle-même affaiblit la toute-puissance du male gaze en concédant la possibilité d'un « transvestisme métaphorique $\gg$ dans lequel la spectatrice peut s'identifier au masculin pour dépasser la soumission de sa position originelle. La fermeture des textes supposée par le formalisme de la sémiotique structurale a produit la nécessité de repenser l'épistémologie et la méthodologie de l'analyse des représentations des femmes dans les médias, pour passer d'une analyse qui clôt le sens des textes sur eux-mêmes au constat de leur polysémie et de leur ambiguïté, 
textes désormais envisagés comme participant de la lutte de définition du monde. S'attardant sur la conflictualité des représentations, l'étape post-structurale est largement influencée par la redéfinition foucaldienne du pouvoir qui, pluralisé et éclaté, s'exprime au niveau microscopique et produit forcément de la résistance.

Ce sont les cultural studies dans leur mouvance féministe qui ont véritablement permis de comprendre les luttes de définition que portent les fictions, en s'attachant tout particulièrement à la fonction idéologique des médias. L'élan des cultural studies est décisif grâce à son analyse à la fois post-structuraliste et constructiviste des liens entre culture et pouvoir dans les représentations médiatiques et dans les industries culturelles. En s'éloignant du fonctionnalisme qui a longtemps prévalu, les cultural studies ont pris un tournant important en envisageant les fictions comme participant des controverses de la sphère publique, à l'échelle d'imaginaires socioculturels qu'elles ont cessé de détacher et d'opposer à un « réel » social. En se détournant d'une approche des médias de masse comme miroirs déformants de la réalité, les travaux féministes ont ainsi pu appréhender les processus médiatiques de négociation, de compromis, de construction. Les travaux d'Éric Maigret et d'Éric Macé sur les médiacultures, néologisme qui dépasse la traditionnelle opposition entre les médias et la Culture, ont importé en France les réflexions anglo-saxonnes des cultural studies, mais ils ont également contribué, les premiers, à repenser, dans un espace français particulièrement structuré par les théories bourdieusiennes qui postulent une correspondance entre domination économique et domination culturelle, les « rapports entre communication et pouvoir [pour redéfinir] un espace de formation et de circulation des paroles et des identités $^{59} \gg$. Le paradigme médiaculturel est celui d'un « constructivisme conflictualiste » qui lie les représentations (dont la capacité signifiante est réhabilitée) avec les rapports sociaux (que les théories postmarxistes du pouvoir permettent de redécouvrir). Cette épistémologie démocratise les constructions de sens dans les pratiques culturelles et, ce faisant, ouvre enfin la voie à une étude renouvelée des politiques de représentation. 


\section{Notes}

1 Robinson, Lilliane, «What's My Line? Telefiction and Women's Work », in Sex, Class \& Culture, Bloomington, Indiana University Press, 1978.

2 Friedan, Betty, La Femme mystifiée, Paris, Gonthier, 1963.

3 Greer, Germaine, La Femme eunuque, Paris, Robert Laffont, 1971.

4 Sullerot, Évelyne, La Presse féminine, Paris, Armand Colin, 1964.

5 Gallagher, Margaret, «Feminist Media Perspectives », in Valvidia, Angharad, A Companion to Media Studies, Hoboken, Wiley-Blackwell, 2005, p. 19-39.

6 Gerbner, George, Signorielli, Nancy, Women and Minorities in Television Drama. 1969-1978, Philadelphie, University of Philadelphia, Annenberg School of Communications, 1979.

7 Van Zoonen, Liesbet, Feminist Media Studies, Londres, Sage Publications, 1994.

8 Steeves, Leslie H., « Feminist Theories and Media Studies », Critical Studies in Mass Communication, 1987, 4, 2, p. 95-135.

9 Tuchman, Gaye, « Women's depiction in the mass media », Signs, 3, 1979 .

10 Van Zoonen, Liesbet, Feminist Media Studies, Londres, Sage Publications, 1994, p. 12.

11 C'est le cas de Liesbet van Zoonen ou Leslie H. Steeves.

12 Une perspective également adoptée par Liesbet van Zoonen, Margaret Gallagher ou Michèle Mattelart.

13 Mattelart, Michèle, « Femmes et médias. Retour sur une problématique $\gg$, Réseaux, 4, 120, 2003, p. 23-51.

14 Coulomb-Gully, Marlène, « Les sciences de l'information et de la communication : une discipline Gender blind ? », Questions de communication, 15, 2009, p. 129-153.

15 Janus, Noreene Z., «Research on Sex-Roles in the Mass Media: Toward a Critical Approach », The Insurgent Sociologist, 1977, 7, 3, p. 19-31.

16 Carson, Diane, Dittmar, Linda, Welsch, Janice R., Multiple Voices in Feminist Film Criticism, Minneapolis, University of Minnesota Press, 1993, p. 34. 
17 Penley, Constance, Feminist and Film Theory, Londres, Routledge, 1988, p. 3.

18 Johnston, Claire, « Women's Cinema as Counter-Cinema », in Johnston, Claire, Notes on Women's Cinema, Londres, Society for Education in Film and Television, 1973.

19 Mulvey, Laura, «Visual Pleasure and Narrative Cinema », Screen, $16,3,1975$.

20 hooks, bell, « The Oppositional Gaze: Black Female Spectators », in Thornham, Sue, Feminist Film Theory: A Reader, New York, New York University Press, 1999, p. 307-320.

21 Creed cite Alien (1986), The Hunger (1983), Carrie (1976), The Brood (1979), Dressed to Kill (1980), The Exorcist (1973), Psycho (1960), Basic Instinct (1992), Whatever Happened to Baby Jane? (1962), A Reflection of Fear (1973), Cat People (1942), Life-Force (1985), I Spit on your Grave (1978).

22 Sellier, Geneviève, « Gender studies et études filmiques », Cahiers dugenre, 2005, 38, 1, p. 63-85.

23 Freeland, Cynthia, «Feminist Film Theory », in Kelly, Michael, Encyclopedia of Aesthetics, Oxford, Oxford University Press, 1998.

24 Modleski, Tania, Hitchcock et la théorie féministe, Paris, L'Harmattan, 2002, p. 10.

25 Lauretis (de), Teresa, Alice Doesn't. Feminism, Semiotics, Cinema, Bloomington, Indiana University Press, 1984, p. 153.

26 Turk, Edward B., Marcel Carné et l'âge d'or du cinéma français, 19291945, Paris, L'Harmattan, 2002.

27 Stacey, Jackie, Star Gazing: Hollywood Cinema and Female Spectatorship, Londres, Routledge, 1994.

28 Rollet, Brigitte, Sellier, Geneviève, « Cinéma et genre en France : état des lieux », Clio, 1999, 10.

29 Spigel, Lynn, Make Room for TV. Television and the Family Ideal in Postwar America, Chicago, University of Chicago Press, 1992.

30 Huyssen, Andreas, After the Great Divide: Modernism, Mass Culture, Postmodernism, Bloomington, Indiana University Press, 1987.

31 Spigel, Lynn, ibid., p. 61.

32 Huyssen, Andreas, ibid. 
33 Burch, Noël, De la beauté des latrines. Pour réhabiliter le sens au cinéma et ailleurs, Paris, L'Harmattan, 2012, p. 24.

34 Huyssen, Andreas, «Féminité de la culture de masse, l'autre de la modernité », in Sellier, Geneviève, Viennot, Éliane, Culture d'élite, culture de masse et différence des sexes, Paris, L'Harmattan, 2004.

35 Sellier, Geneviève, « Images de femmes dans le cinéma de la Nouvelle Vague », Clio, 10, 2, 1992. Disponible en ligne : http://clio.revues. org/265?lang=fr, consulté le 14 septembre 2014 .

36 Péquignot, Bruno, «Les femmes dans le roman sentimental moderne », L'Homme et la société, 99-100, 1991, p. 115-125.

37 Péquignot, Bruno, ibid.

38 Allen, Robert C., Speaking of Soap Opera, Chapel Hill, University of North Carolina Press, 1985.

39 Brunsdon, Charlotte, D’Acci, Julie, Spigel, Lynn, Feminist Television Criticism. A Reader, Oxford, Oxford University Press, 1997.

40 Lopate, Carol, « Daytime Television. You'll Never Want To Leave Home », Radical America, 11, 1977.

41 Modleski, Tania, Loving with a Vengeance. Mass-Produced Fantasies for Women, Londres, Routledge, 1984, p. 108.

42 Modleski, Tania, ibid., p. 106.

43 Geraghty, Christine, Women and the Soap Opera. A Study of Prime Time Soaps, Cambridge, Polity Press, 1990 ; Geraghty, Christine, «The study of the soap opera », in Wasko, Janet, $A$ Companion to Television, Hoboken, Wiley-Blackwell, 2005.

44 Radway, Janice A., Reading the Romance. Women, Patriarchy and Popular Litterature, Chapel Hill, University of North Carolina Press, 1992.

45 Pour un aperçu des enjeux, je renvoie à McCabe, Janet, Akass, Kim, « Feminist Television Criticism: Notes and Queries », Critical Studies in Television, 1, 2006, p. 108-120.

46 Lotz, Amanda, « Postfeminist Television Criticism: Rehabilitating Critical Terms and Identifying Postfeminist Attributes $\gg$, Feminist Media Studies, 1, 2001, p. 105-121.

47 Rowe, Kathleen, The Unruly Woman: Gender and the Genres of Laughter in Understanding Inequality, Austin, University of Texas Press, 1995. 
48 Mayne, Judith, « L.A. Law and Prime-Time Feminism », in Brunsdon Charlotte, D’Acci, Julie, Spigel, Lynn, Feminist Television Criticism. A Reader, Oxford, Oxford University Press, 1997, p. 84-97.

49 Clark, Danae, « Cagney \& Lacey: Feminist Strategies of Detection », in Brown, Mary Ellen, Television and Women's Culture: The Politics of the Popular, Washington DC, Sage Publications, 1990, p. 116-133.

50 Van Zoonen, Liesbet, ibid.

51 Hall, Stuart, « The Problem of Ideology. Marxism without Guarantees », Journal of Communication Inquiry, 1986, vol. 10, n 28, p. 28-44.

52 Macé, Éric, L'après-patriarcat, Paris, Seuil, 2015.

53 Mattelart, Michèle, « Femmes et médias. Retour sur une problématique », Réseaux, 4, 120, 2003, p. 23-51.

54 Fiske, John, Television Culture, Routledge, 1994.

55 Morin, Edgar, L'Esprit du temps, Paris, Armand Colin, 2010. Sur la question de l'ambivalence et de l'ambiguïté, citons également les travaux de Noël Burch autour du «double speak » hollywoodien : Burch, Noël, «Double Speak. De l'ambiguité tendancielle du cinéma hollywoodien », Réseaux, 2000, 99, p. 99-130.

56 Hall, Stuart, Identités et cultures. Politiques des cultural studies, Paris, Amsterdam, 2008.

57 McRobbie, Angela, "Jackie": an Ideology of Adolescent Femininity, Birmingham, University of Birmingham, 1978.

58 Mulvey, Laura, « Afterthoughts on "Visual Pleasure and Narrative Cinema” inspired by King Vidor's Duel in the Sun (1946) », Framework, 15-16-17, 1981, p. 12-15.

59 Maigret, Éric, Macé, Éric, Penser les médiacultures. Nouvellespratiques et nouvelles approches de la représentation du monde, Paris, Armand Colin, 2005, p. 13. 



\section{Réconcilier l'individualisme et le féminisme et comprendre les subjectivités post-traditionnelles}

\section{Constructions et luttes internes du féminisme de la deuxième vague}

Comprendre les subjectivités des héroïnes de séries télévisées demande de comprendre les constructions et luttes internes des courants féministes, et notamment de la deuxième vague. Après une première vague centrée sur les égalités de droit au début du $\mathrm{Xx}^{\mathrm{e}}$ siècle, combat des fameuses suffragettes, le féminisme amorce un deuxième mouvement pour l'égalité de fait, à partir de la fin des années 1960. Celui-ci vise à remettre en question le patriarcat comme système basé sur la distinction sexe/genre et sur l'exploitation domestique des femmes. Dans le remarquable historique des féminismes qu'il produit à l'occasion d'une réflexion sur Les Féministes et le garçon arabe avec Nacira Guénif-Souilamas, Éric Macé souligne que la lutte fondatrice du féminisme est celle contre la différentiation hiérarchisée et fonctionnelle entre hommes et femmes justifiée par Dieu et la nature, confiant aux premiers la production et aux secondes la reproduction. Or, les valeurs démocratiques sont érigées par la modernité « en principe dominant de la vie politique ${ }^{1} \gg$, puis réappropriées et scandées par les féministes en vue d'un « féminisme égalitariste et civique ${ }^{2}$ ». Y succède un « féminisme politique » lancé par Le Deuxième sexe de Simone de Beauvoir en 1949, qui s'attache à déconstruire la « nature » du féminin pour en révéler les 
dynamiques culturelles. De la première à la deuxième vague, les contrehégémonies suscitent des contre-offensives patriarcales. Leurs modalités discursives et politiques s'adaptent l'une à l'autre et se déplacent : tandis que les suffragettes plaident pour l'égalité au nom des principes démocratiques de la modernité, les idéologies patriarcales mobilisent les arguments « naturels » pour renvoyer les femmes à leur fonction reproductive. Ce sexisme biologique rappelle les idéologies naturalisantes qui se sont exprimées dans le racisme ${ }^{3}$. La deuxième vague féministe apporte, avec les réflexions pionnières de Simone de Beauvoir sur la construction du genre, une disqualification de ces arguments « naturels », qu'enregistre l'idéologie patriarcale. Comme le souligne bien le travail sociohistorique d'Éric Macé, le sexisme opère alors un déplacement de la catégorie de la nature vers celle de la culture, ainsi qu'a pu l'analyser, là aussi, Paul Gilroy dans son travail sur l'usage de discours essentialistes et anti-essentialistes dans les controverses sur la « race $^{4} »$. La menace sexiste qui plane désormais sur les femmes est de renoncer à ce qui fait leur force, la féminité, et de devenir agressives et esseulées. Éric Macé plaide pour une réconciliation du féminisme égalitariste portant ses efforts sur la législation, les structures, les organisations - bref sur le droit - avec un « déconstructivisme queer », pour qu'explosent dans une démultiplication infinie les genres et les sexualités.

Au sein cette deuxième vague, les historiens s'accordent à distinguer deux courants majeurs à la fin des années 1960. Le premier est le féminisme libéral (liberal feminism), dont le mouvement étasunien National Organisation for Women (NOW) fondé par Betty Friedan, Pauli Murray et Shirley Chisholm est le plus représentatif. Réformiste, ce courant est pour Marie-Hélène Bourcier un «féminisme des droits [dont les] demandes sont sectorielles et ne remettent pas en cause la société de manière systémique $^{5} \gg$. Il voit dans l'exclusion des femmes de la sphère publique le principal obstacle aux inégalités de genre et se détourne ainsi des problématiques privées, ce qui rend ses puissances d'agir très limitées selon l'historienne des genres Ruth Rosen ${ }^{6}$. Ce constat est partagé par Valerie Bryson, professeure de théorie politique, qui considère que cette priorité donnée à la sphère publique est en fait un oubli voire une invisibilisation des effets de pouvoir qui traversent la vie privée des individus. Le second courant est celui du Mouvement de Libération des Femmes (MLF en France, dont l'équivalent étasunien est le Women's Lib) qui doit être compris au miroir des grandes 
luttes pour les droits civiques. Ce féminisme radical (radical feminism) nourrit un espoir révolutionnaire, en opposition avec le réformisme du féminisme libéral, et bouleverse la dichotomie privé/public en pensant le personnel comme politique. Les événements contenus dans la sphère privée ne relèvent pas de décisions individuelles ni personnelles mais sont étroitement articulés avec les sphères politiques, les institutions et les rapports sociaux, que ce soit du côté de la législation, de la famille, de la sexualité ou de la division du travail. Ce mouvement n'est pas sans sous-divisions ni dissensions internes, comme le montrent bien les désaccords entre la mouvance pro-sexe et la lutte anti-pornographie menée par Catharine MacKinnon ${ }^{7}$, ou entre la volonté de déconstruire le genre féminin et sa célébration dans le courant du néoféminisme en France ou du cultural feminism (la terminologie est de l'historienne Alice Echols ${ }^{8}$ ). Le féminisme radical sort plus affaibli de cette confrontation que le féminisme libéral. Selon Flora Davis, « en 1975, la plupart des groupes de libération des femmes s'étaient évaporés ${ }^{9} \gg$ et l'organisation NOW occupait les espaces vacants, étalant son influence jusqu'à la scène politique de Washington.

L'influence du marxisme sur le féminisme est forte, notamment chez les féministes matérialistes comme Christine Delphy qui ont produit une théorie économique du système patriarcal. Des outils marxiens comme la division du travail ou les rapports de production ont permis d'éclairer les mécanismes par lesquels « les femmes sont exclues du marché (del'échange) en tant qu'agents économiques, et non leur production », pour preuve les différentes valorisations du travail ménager selon qu'il soit réalisé par une professionnelle, femme de ménage, ou par une mère au foyer ${ }^{10}$. Pour Monique Wittig, autre grande figure du féminisme matérialiste, les rapports de production marxiens éclairent l'exploitation des femmes par les hommes, sur le modèle de celle des ouvriers par les bourgeois ${ }^{11}$. Aliénation et exploitation viennent structurer ces rapports que les femmes ont intériorisés et aux exigences desquelles elles se soumettent, l'analogie que fait Wittig de la féminité des femmes avec l'étoile jaune des Juifs ${ }^{12}$ constituant un exemple violent du manque d'actualisation de concepts marxiens devenus marxistes, élaborés presque deux cents ans plus tôt. La lutte des sexes n'est soluble que dans le refus radical des constructions patriarcales de la féminité et de l'hétérosexualité (toutes deux étant articulées). La proposition de Wittig d'une «avant-garde lesbienne » crée une menace radicale: 
« non seulement les féministes contrarient la nature des femmes, non seulement elles les “déféminisent", mais en plus elles en font les ennemies agressives des hommes dans la vie sociale, affective, sexuelle et personnelle ${ }^{13} \gg$. La charge est lancée, qui aura des conséquences politiques majeures, tant du côté des idéologies patriarcales qu'au sein du féminisme de la deuxième vague lui-même. Le retour à l'argument « naturel » est néanmoins enclenché avec le néoféminisme qui, réhabilitant la causalité sexe-genre, opère un retournement de stigmate des plus essentialistes en célébrant les qualités dites féminines. Celui-ci conduit à « une défense et une illustration du “génie”, du "bonheur", du “pouvoir”, des “qualités" féminines ${ }^{14} \gg$.

\section{L'ambiguïté des redéfinitions des corps par le néoféminisme}

En parallèle du féminisme de la deuxième vague, un néoféminisme ambigu se développe, dont les médiations médiatiques portent largement sur les corporalités des héroïnes. Clé de voûte de l'indépendance féminine, le corps a été au cœur des réflexions féministes des années 1970 : longtemps défini par la naturalisation patriarcale qui le réduit à sa fonction reproductive, il a été placé au cœur des enjeux féministes, tant par le néoféminisme qui revalorise ses qualités jugées essentielles que par le féminisme de la troisième vague luttant précisément pour en déconstruire la supposée naturalité. Les imaginaires médiatiques interrogent ces mouvements féministes, jouant de procédés comme l'ironie, la symbolisation ou le carnavalesque. La sexualité et l'objectivation des femmes restent les caractéristiques les plus critiquées, les recherches prenant souvent appui sur les travaux de Laura Mulvey relatifs au male gaze ${ }^{15}$. Mulvey défend que, sous le contrôle de l'hétérosexualité masculine, les caméras scopophiles rendent les femmes passives et lascives. Ainsi objectivées en fonction de critères esthétiques masculins hétérosexuels, elles n'acquièrent jamais le statut de sujet. Impossible d'échapper à ce regard, impossible également de se le réapproprier, sinon en endossant temporairement la position masculine, ce qui équivaut à entrer dans le piège de sa légitimation. Au-delà des dérives hétéronormées de cette théorie (une femme ne peut-elle, sans qu'il soit question d'aliénation ou de pièges du masculin, apprécier regarder le corps d'une autre femme ?), le male gaze reste précieux en ce qu'il a permis de formaliser pour la première fois le 
rôle des corps féminins dans la production des imaginaires visuels comme objets esthétiques et non comme sujets actifs.

Des femmes au foyer des années 1950 jusqu'aux femmes actives, des personnages les plus passifs aux héroïnes les plus travailleuses, la beauté des personnages est rarement altérée ou problématisée : Donna Reed, Samantha Stephens, Mary Richards, les Drôles de Dames, Carrie Bradshaw, Alicia Florrick... bon nombre des héroïnes sont belles et élégantes, d'une féminité réconfortante. Mais la systématicité cachant souvent l'incomplétude, il faut immédiatement ajouter à ce tableau l'héroïne prolétaire et corpulente Roseanne, bien éloignée des idéaux des années 1950 et 1960. Dépeignant de façon franche le quotidien de la ménagère américaine, Roseanne Barr, elle-même issue d'une famille d'immigrés ouvriers juifs, avait pour ambition explicite de proposer une alternative réaliste aux femmes au foyer de classe moyenne. En jeans et chemises larges, loin de la minceur que soulignent les jupons des années 1960, Roseanne subvertit les représentations traditionnelles d'un corps féminin au service du foyer familial et montre que la domesticité est, de façon très pratique, antithétique de la féminité classique, ajoutant à cette équation une variable de classe évidente et pourtant souvent invisibilisée : l'héroïne aurait-elle l'envie et le temps d'élaborer une féminité, l'argent lui manquerait. Les performances de Roseanne tendent à confirmer que, lorsque les corps ne sont pas contenus, ils deviennent dangereux. En ce sens, Katherine Lehman a montré que jusqu'aux années 1970, les sexualités des femmes sont considérées comme un risque : dans That Girl!, les hommes qui veulent séduire Ann Marie

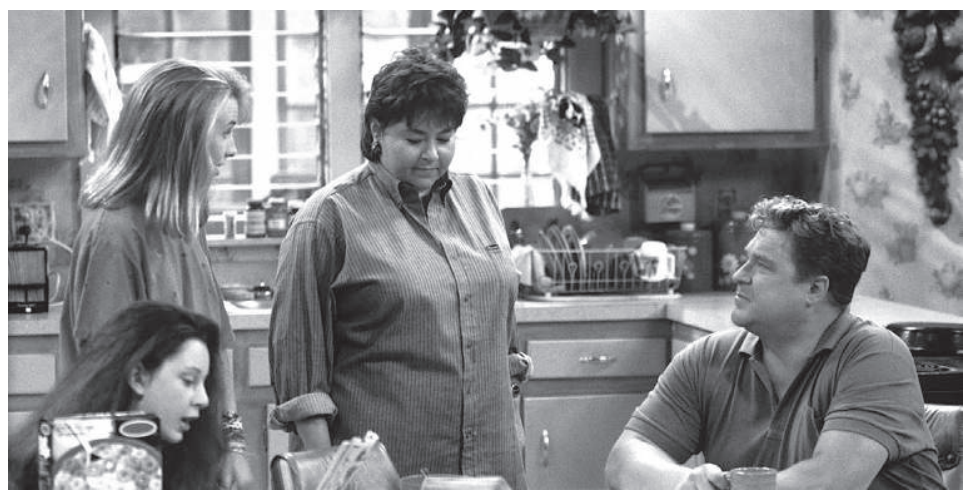


semblent n'être là que pour réaffirmer la chasteté de l'héroïne : ne vous en faites pas, « elle n'est pas “ce genre" de fille ${ }^{16}$ ». De la même façon, dans Peyton Place, un premier rendez-vous amoureux se termine mal pour Betty, lorsque l'homme qu'elle a fréquenté durant la soirée, et qui pense pouvoir coucher avec elle, lui court après dans sa chambre jusqu'à ce qu'il réalise qu'elle n'est pas en train de jouer mais le fuit vraiment. Le dialogue est lapidaire lorsqu' il lui conseille de faire ses affaires et de rentrer dans sa province car « le prochain garçon ne sera peut-être pas aussi raisonnable » que lui. Cette idéologie prudente, sinon prude, est aussi présente dans The Mary Tyler Moore Show, où la sexualité de l' héroïne reste implicite, contenue à de rares allusions contraceptives ou à des « derniers verres » consommés dans son appartement après des rendez-vous amoureux. Les femmes ont beau être intelligentes et autonomes, leur corps continue de les mettre en danger sans qu'elles n'aient la possibilité d'en offrir des redéfinitions. Ils sont les obstacles les plus cristallisés à l'émancipation, comme des rappels naturels (en fait, naturalisés) des possibilités et des limites de l'être féminin. Quoi de plus naturel, quoi de plus objectivement naturel, après tout, que ces tissages cellulaires et ces organes mécaniques ? Face aux assauts émancipateurs de l'individualisme et du féminisme alliés, la différence sexuelle se réfugie dans des corps qu'elle construit comme des données naturelles inaliénables. Récupérant le mythe rousseauiste, l'idéologie patriarcale exalte la fonction maternelle, sa douceur, sa beauté, sa compréhension, jusqu'à la recoder comme essentiellement supérieure dans un néoféminisme qui n’a plus de féministe que la généalogie.

Quand émerge dans les années 1970 le postféminisme que Dow définit par l'individualisme et le primat du choix personnel, et auquel McRobbie ajoute le « contrat sexuel ${ }^{17} \gg$, soit une re-féminisation rassurante en échange de l'accès à la sphère publique, il est accompagné de tonalités néoféministes dont les Drôles de Dames sont les plus significatives, et qui perdurera jusqu'aux héroïnes de l'après-féminisme des années 1990 comme Ally McBeal ou Carrie Bradshaw. En se réappropriant les corporalités et les sexualités jusqu'ici confisquées ou redoutées, le néoféminisme a pour tactique le retournement de stigmate mais ne suit pas un processus de déconstruction comme cela a pu être le cas, comme l'a bien montré Stuart Hall, dans des enjeux de race et d'ethnicité. Au lieu de cela, ces balbutiements néoféministes ont au contraire honoré de plus belle les qualités féminines : 
ils ont revendiqué l'accès à la sphère publique au nom de caractéristiques et de valeurs exclusivement féminines, ont célébré la complémentarité dans les relations amoureuses, ont naturalisé la maternité ou encore se sont arrogé le privilège parental ${ }^{18}$. Une telle stratégie se veut d'autant plus valorisante pour les femmes qu'elle dénonce, comme le montre bien Éric Macé, des qualités et des valeurs codées masculines, considérées comme violentes et oppressantes et dont seraient symptomatiques les formes sexuelles libérées comme la prostitution ou la pornographie ${ }^{19}$. Le néoféminisme reste enlisé dans une stratégie de retournement hégémonique plutôt que de se transformer en « essentialisme stratégique » tel que l’a défini Gayatri Chakravorty Spivak, pour qui les subaltern studies opèrent un tournant lorsqu'elles envisagent les mutations sociales comme un processus conflictuel plutôt que transitionnel, privilégiant les concepts marxiens de domination et d'exploitation en lieu et place de la vision évolutionniste des modes de production. La conséquence de ce déplacement est que « la puissance d'agir du changement est située dans l' insurgent ou dans le "subalterne" ${ }^{20} »$, dont les actions peuvent servir la déconstruction de son oppression. Les subalternes peuvent ainsi faire « un usage stratégique de l'essentialisme positiviste en vue d'une scrupuleuse visibilité politique ${ }^{21} \gg$, mettant de côté leurs différences internes pour proposer dans la sphère publique une représentation standardisée et fédérée d'eux-mêmes, réutilisant à leur compte les caractéristiques qui leur ont été apposées pour en montrer les logiques oppressantes. Parce que les héroïnes très féminines ne passent pas la vitesse supérieure de la déconstruction, l'« essentialisme stratégique » se limite à l'essentialisme. Au fur et à mesure de la montée des problématiques réflexives, la féminité se charge toutefois d'une mise à distance voire d'une ironie qui rend visible la performance du genre. Le néoféminisme, s'il reste certes prisonnier du premier stade de retournement de stigmate, ne sort pas indemne de l'ère de la réflexivité généralisée. À défaut d'opérer une déconstruction du genre, le recul qu'il prend sur les codes féminins rend compte de la construction socioculturelle de ces derniers. Comme le souligne Katherine Lehman, les premières manifestations médiatiques du néoféminisme servent la revendication d'un pouvoir féminin jusqu'ici endigué par les figures patriarcales, puisque « l'autonomie relative et l'usage stratégique de la sexualité par ces femmes [font de] leur charme une source de pouvoir et d'influence dans l'environnement professionnel, plutôt qu'un lieu de victimisation 
ou d'objectivation ${ }^{22} \gg$. Avec toute l'ambiguïté qui le caractérise, le néoféminisme, tantôt retournement de stigmate, tantôt réaffirmation de la différence sexuelle, s'insère particulièrement bien dans le syncrétisme de médias de masse ainsi enclins à « négocier des tensions entre féminisme et antiféminisme ${ }^{23} \gg$. Dans des sociétés individualistes et capitalistes où le capital esthétique oscille entre objectivation et souci de soi, dans une deuxième modernité qui promeut et « dé-genre » les qualités codées féminines comme le care et la communication, la corporalité reste le territoire privilégié où s'exprime un néoféminisme majoritairement assignant, même s'il reste traversé de logiques émancipatrices.

Au milieu des années 1970, un ensemble de séries émancipe les corps en même temps qu'il déplace les hérö̈nes du registre de la comédie vers le drama, les habillant pour la première fois des uniformes traditionnellement masculins : les femmes sont policières, détectives ou super-héroïnes. Avec Police Woman (1974-1978), Get Christie Love! (1974-1975), Wonder Woman (1975-1979) ou encore Drôles de Dames (1976-1981), l'angoissante question se pose au patriarcat des bouleversements qu' induit l'arrivée des femmes dans la sphère professionnelle. L'hypothèse de Lehman est que le mouvement de féminisation des héroïnes et l'utilisation explicite de leurs charmes contrecarrent la menace androgyne que fait planer le féminisme sur le système de genre. On retrouve cette idée dans ce que Angela McRobbie a récemment appelé la « mascarade postféministe ${ }^{24}$ », actualisation du backlash décrit par Susan Faludi ${ }^{25}$. Les femmes seraient au cœur d'un « contrat sexuel » engagé par le gouvernement et les institutions, contrat qui troque protection et égalité des chances (en réalité toutes relatives) contre un complexe de beauté et de mode ne faisant que réaffirmer les caractéristiques genrées : en d'autres termes, pour obtenir l'égalité de droit qui leur donne théoriquement un accès équitable à la sphère professionnelle, les femmes ont concédé, d'une part, la pérennisation de la distinction masculin/féminin, et d'autre part, la reconfirmation des identités féminines telles que l'idéologie patriarcale les a définies. Bridget Jones ou Carrie Bradshaw ont beau être des journalistes ambitieuses et reconnues, leur féminité rassure les dichotomies traditionnelles et prévient tout trouble dans le genre. Si McRobbie tire un modèle de ce complexe, l'analyse de Lehman a ceci de plus riche qu'elle intègre une compréhension des dynamiques de réappropriation des corps ainsi qu'une 
contextualisation des enjeux socio-politiques d'alors. Car, en ces temps où la société étasunienne est aux prises avec l'Equal Rights Amendment qui propose que « l'égalité des droits en vertu de la loi ne sera déniée ou limitée par les États-Unis ni par aucun état en raison du sexe », la sphère publique regorge de débats sur l'égalité des genres, leurs différences ou leurs complémentarités. Dans les mois qui suivent, la discussion s'enrichit des soucis de harcèlement sexuel quand des associations féministes comme Working Women United ou Alliance Against Sexual Coercion lancent l'assaut contre les avances indésirables faites aux femmes, dans la rue ou au travail, et focalisent le débat sur l'appropriation des corps peu après que la pilule a été autorisée (1970) et peu avant que l'avortement ne le soit (1973). Droit de disposer de son corps, priorité de l'identité individuelle féminine sur la potentielle mère, éclairage du harcèlement sous-jacent à ce qui a été, stratégie euphémisante, défini comme « flirt » ou « jeu de séduction »... voilà autant de problématiques qui sont en fait au cœur des récits d'hérö̈nes d'action des années 1970, principalement policières ou détectives qui utilisent leur beauté comme arme. De l'afro-américaine Christie (Get Christie Love!) à la policière en détresse Pepper (Police Woman), en passant par les super-pouvoirs de Wonder Woman ou de The Bionic Woman, toutes ces héroïnes ont des talents qui reposent sur leur autonomie et leur capacité d'agir mais aussi sur leurs atouts physiques. Pour la première fois, la sexualité des héroïnes n'est pas passée sous silence ni contenue à la sphère publique, mais remobilisée comme une force spécifiquement féminine, et ce sans tolérer la moindre récupération masculine sous forme d'avances, de harcèlements ou d'attaques sexuelles. Dans ces séries, de nombreux épisodes traitent des agressions subies par les femmes, qu'il s'agisse de personnages périphériques ou des héroïnes elles-mêmes. Par ricochet, les personnages masculins deviennent plus hétérogènes, préservant la figure du patriarche ou du sauveur par endroits comme dans Police Woman, ou évoquant ailleurs des machos que la solidarité féminine se charge de faire tomber (Drôles de Dames).

Néanmoins, cette lecture stratégique de la sexualisation des héroïnes n'est, à l'époque, pas la plus partagée. Pressées par des plaintes, les chaînes de télévision sont priées en 1978 de ne plus représenter de telles héroïnes, pour privilégier à la place des femmes aux charmes moins affirmés, mais aussi moins affirmatifs ${ }^{26}$. On trouve des traces de ces controverses dans 
les milieux institutionnels et dans des articles de presse. En 1977, un rapport intitulé «Window Dressing on the Set: Women and Minorities in Television » de la Commission américaine pour les droits civiques (U.S. Commission on Civil Rights) demande à la Federal Communications Commission, responsable de réguler les industries médiatiques, de veiller à ce que les minorités soient mieux représentées. Le rapport tirait une relation de cause à effet entre la sous-représentation des minorités dans les chaînes d'écriture et de production et la surreprésentation de caractéristiques les stigmatisant dans les représentations. Un journaliste du Washington Post résume ainsi la conclusion du rapport : « la télévision offre une vision du monde dominée par le point de vue de l'homme blanc car les hommes blancs dominent la structure qui définit la télévision ${ }^{27} \gg$. Le syndicat Screen Actors' Guild entre en 1978 dans le débat lorsque sa présidente évoque lors d'un panel sur la monstration des minorités l'image « dégoûtante et déshonorante [qui se veut être celle] de la femme américaine ${ }^{28} \gg$. Il est intéressant de voir que les plaintes contre ce qui est perçu comme une objectivation univoque des femmes sont formulées par de nombreux acteurs de part et d'autre de l'échiquier politique. Sur le sujet, des groupes d'ordinaire opposés s'accordent : associations conservatrices, mouvements féministes, publicitaires, critiques, scientifiques, producteurs de télévision et même interprètes déplorent ces représentations et plaident auprès des chaînes pour qu'elles soient infléchies, sans qu'il y ait trace quelque part de manifestations similaires de la part des publics. Les héroïnes féminines et charmeuses sont, à partir des années 1980, peu à peu rayées des scénarios de télévision, au nom des effets sexistes qu'elles produiraient, sans que s'expriment ni ne soient consultés les téléspectateurs.

\section{L'individualisme écartelé entre théories féministes et théories de la réflexivité}

L'apparition, à l'orée des années 1970, d'héroïnes qui sont conjointement responsables de leurs actions et ouvertement aux prises avec des problèmes féministes pose des questions à un grand partage défendu par d'importantes auteures comme Angela McRobbie ou Bonnie Dow. Pour ces dernières, la modernité avancée est l'époque d'individus égocentrés voire égoïstes, entraves aux développements du féminisme. D’un côté, le 
féminisme serait un mouvement collectif qui, s'il s'exprime dans les vies individuelles, ne peut le faire que selon le principe ascendant de la diffusion des luttes macrosociales dans l'expérience microsociale. De l'autre, l'individualisme serait un mouvement d'atomisation et d'isolement des individus, faisant naître une théorie de la modernité avancée dans laquelle l'influence des structures est injustement amoindrie et celle des actions individuelles, magnifiée. L'individualisme apparaît comme écartelé entre les théories féministes et les théories de la réflexivité. Or, le problème qui est posé de l'essor à priori impossible du féminisme dans la modernité avancée provient d'une définition de l'un et de l'autre qui apparaît trop restreinte. Les héroïnes de séries télévisées obligent justement à saisir les limites du rapport des féminismes à l'individualisme, ainsi qu' à comprendre ce que la réflexivité produit comme nouvelles subjectivités, pour réinterroger cette dichotomie entre actions strictement individuelles (individualisme) et luttes obligatoirement collectives (féminisme). Il faut d'ailleurs souligner que cette opposition entre individualisme et féminisme est l'objet de contestations au sein même des mouvements féministes. Liesbet van Zoonen ne partage pas les positions d'Angela McRobbie. Comme elle le formule bien, «l'absence d'identités fixées et l'articulation sans cesse reformulée de la différence qui en résulte ne sape pas les solidarités et les politiques au sens traditionnel, comme cela est souvent craint, mais peut encourager une variété d'alliances contre les nombreuses configurations de pouvoir ${ }^{29} \gg$. À relire les auteurs qui ont conceptualisé les premiers la modernité réflexive (Ulrich Beck, Anthony Giddens) ou ceux qui y ont substantiellement contribué (Kaufmann, de Singly, Latour), l'individualisme contemporain se révèle bien différent de ce qu'en ont retenu les féministes critiques. On ne trouve aucune trace d'une obsolescence des structures, d'une responsabilisation excessive des individus ou d'une célébration d'une réflexivité définie comme hyper-conscience dans l'ouvrage fondateur de cette théorie, Reflexive Modernization. Politics, Tradition and Aesthetics in the Modern Social Order (1994), sinon pour les désavouer formellement. Lire dans la théorie de la modernité avancée la disparition du poids des structures, la fin des luttes collectives et l'atomisation du politique, c'est comprendre tout l'inverse de ce qui a motivé son élaboration, c'est aussi confondre modernité réflexive et post-modernité alors que la première est née en réaction à la seconde. Tandis que la post-modernité postule la déconstruction tant des 
structures (dé-structuration) que des sciences (dé-conceptualisation), la modernité réflexive défend la re-structuration et la re-conceptualisation. Celles-ci constituent tant le cœur de la modernité réflexive que Bruno Latour suggère de renommer cette dernière « re-modernisation ${ }^{30} \gg$.

Une posture relativement similaire se retrouve dans la proposition d'un « après-patriarcat » tel que défendu par Éric Macé, à l'occasion d'une réflexion très précise sur les logiques articulées de détraditionnalisation et de réagencements de rapports et identités de genre. L'auteur y interroge une tension contemporaine entre « un principe d'égalité authentiquement partagé et la fabrique collective d'inégalités ${ }^{31} \gg$, ce qu'il nomme «l'égalitarisme inégalitaire paradoxal », nous enjoignant de séparer la question des inégalités de genre contemporaines de leur légitimation par un patriarcat dont les conditions d'existence sont réagencées par des tensions internes et sous l'effet des luttes féministes. Soulignant le passage d'un patriarcat traditionnel à un patriarcat moderne, au tournant du $\mathrm{XVIII}^{\mathrm{e}}$ siècle, puis du patriarcat moderne à un post-patriarcat caractérisé par la perte de légitimité de l'asymétrie entre masculin et féminin à compter de la deuxième moitié du $\mathrm{Xx}^{\mathrm{e}}$ siècle, Éric Macé éclaire de nouvelles tensions collectives et subjectives dont l'enjeu conflictuel central est celui de « l'approfondissement de la détraditionnalisation des dimensions genrées de la vie sociale, de la vie sexuelle et des identités, ou de la résistance à cette détraditionnalisation » - autant de thématiques que ce livre ambitionne de débusquer dans les imaginaires médiaculturels.

Sa proposition d'un « après-patriarcat » apparaît en ce sens comme l'une des plus satisfaisantes aujourd'hui en ce qu'elle clarifie l'enjeu scientifique de la compréhension des rapports amoureux contemporains en réactualisant les questions et les concepts à disposition. Il s'agit bien de comprendre les longs et complexes processus de détraditionnalisation qui sont en jeu, tant dans les « rapports et identités de genre », c'est-à-dire dans le genre entendu comme un rapport social que dans les genres entendus comme performances, tous continuant de porter l'histoire patriarcale sans n'y être plus totalement subordonnés. En ce sens, le patriarcat apparaît comme un terme flou en ce qu'« aucune liste de traits empiriques ne pourrait permettre de recouvrir la diversité de ses configurations dans ses formes historiques et contemporaines ${ }^{32} \gg$. Subséquemment, Éric Macé éclaire les impasses théoriques et empiriques de mouvements féministes matéralistes qui voient 
dans l'abolition de la division genrée une sortie, en réalité toute marxiste, de l'histoire cette fois-ci patriarcale. À cela, Éric Macé oppose la stratégie de Judith Butler pour qui il ne s'agit pas de faire disparaître le genre mais de « faire des identifications de genre autant de devenirs possibles ${ }^{33} »$. Cela se produit en resituant socialement le genre selon une triple perspective : « comme une identification sociale qui intègre, comme une différence culturelle sans conséquences, comme une discrimination culturelle qui hiérarchise ${ }^{34} \gg$. Dans la lignée des auteurs de la réflexivité, Éric Macé rejette donc toute visée téléologique pour penser, dans la complexité sociohistorique des rapports de pouvoir entre rejets, résidus, héritages et contemporanéités forcément diverses, un après-patriarcat qui n'est donc « pas postmoderne mais [qui] participe bien au contraire des tensions propres à la seconde modernité, qui sont celles des conséquences de la modernité elle-même ${ }^{35} \gg$.

La difficulté qu'il y a parfois à comprendre que « post » ne signifie pas que l'histoire est terminée transparaît par ailleurs sous une forme de sentiment de dépossession dans les écrits d'Ulrich Beck qui, au cours d'une mise au point sur son modèle, regrette que l'individualisme soit dévoyé de la sorte : le concept, prévient-il, « ne veut pas dire beaucoup des choses qu'un certain nombre de personnes pensent qu'il veut dire, de telle sorte qu'il ne veut plus dire grand-chose ${ }^{36} \gg$. Il me semble crucial de revenir sur le paradigme de la modernité avancée, dont les nombreux synonymes (modernisation de la modernité, radicalisation de la modernité, modernité réflexive, deuxième modernité, modernisation réflexive, re-modernisation...) ne facilitent pas toujours l'intelligibilité. Ce retour doit se faire en suivant deux principes. Le premier est de ne pas céder à la tentation téléologique et évolutionniste : Beck et Giddens rappellent continuellement qu'un monde réflexif n'est pas mécaniquement un monde meilleur, et que la post-tradition ne signifie pas que la tradition ne produit plus d'effets. Le second principe, qui est lié au premier, est le déplacement d'une vision en termes d'oppositions, de ruptures franches ou d'étapes consécutives qui récuse tout dialogue entre tradition et post-tradition, entre individualisme et féminisme ou entre personnel et collectif, vers une pensée des phénomènes d'articulation à l'œuvre dans les grandes mutations sociales du $\mathrm{XX}^{\mathrm{e}}$ siècle : montée des contre-publics subalternes et formalisation des mouvements féministes, émancipations individuelles et libéralisations des mœurs, rationalisation et problématisation des risques amoureux. 


\section{Dialogies entre tradition et post-tradition}

Pour éviter toute déformation, revenons aux sources. Le passage de la modernité à la modernité réflexive s'est effectué, selon Ulrich Beck, par un nouveau rapport aux structures sociales de l'après-guerre qui définissait le modèle classique de la société, parmi lesquelles l'État-nation et providence, les classes sociales et la famille nucléaire. Celles-ci étaient soutenues et venaient soutenir toute la « toile de sécurité économique, tissée par la régulation industrielle, le plein-emploi et les carrières à long terme ${ }^{37} \gg$. Les émancipations des minorités durant la deuxième moitié $\mathrm{du} \mathrm{XX}^{\mathrm{e}}$ siècle s'inscrivent directement dans ce bouleversement de l'ordre social qui n’anéantit pas les institutions mais les rend fluctuantes. Dans le chapitre « Living in a Post-Traditional Society », Anthony Giddens s'attache à définir et décrire les liens entre tradition, modernité et modernité avancée. Partant du principe que la « modernité a reconstruit la tradition au fur et à mesure qu'elle l'a détruite $^{38} »$, Giddens ne cède jamais à l'évolutionnisme et préfère explorer les articulations, oppositions et contradictions, ainsi que les superpositions et les échanges entre ces différentes époques. Son apport majeur est d'offrir une définition de la tradition qui ne soit pas celle en négatif de la modernité, ce qui a été, à son sens, les erreurs à la fois de la sociologie et de l'anthropologie. Selon lui, leurs définitions de la tradition sont en effet insatisfaisantes car la première a été trop préoccupée par la modernité, et la seconde, dans sa perspective fonctionnaliste, a résumé la tradition à la répétition (des rôles, notamment) et à la cohésion sociale. Les individus de l'époque traditionnelle ont été compris, non pas replacés dans leur époque, mais en creux de la modernité, définis comme des sujets passifs, « suivant mécaniquement des préceptes qu' ils acceptent sans les interroger ${ }^{39} \gg$. Cette exagération de la passivité des acteurs de la tradition vient exalter en retour la supposée activité des individus réflexifs. En la matière, d'ailleurs, le panorama que ce présent ouvrage s'efforcera d'élaborer des imaginaires médiatiques des années 1950 et de leurs représentations des femmes au foyer montre bien que les héroïnes « traditionnelles » (ILove Lucy, Donna Reed, Ma Sorcière bien-aimée), bien qu'elles soient insérées dans des structures amoureuses classiques, font preuve de résistances : il serait bien malaisé de qualifier Lucy Ricardo, héroïne du début des années 1950, de « passive », elle qui passe la plupart des épisodes à fuguer du foyer familial. 
Aussi, si la modernité met la tradition à mal, il faut comprendre qu'il se produit entre les deux époques une collaboration, laquelle ne s'est éteinte qu'à la radicalisation de la modernité, laissant place à d'autres échanges entre modernité et modernité avancée. La définition que produit Giddens de la tradition, en cinq points, permet de voir ces superpositions : la tradition est selon lui étroitement liée à la « mémoire collective » selon l'expression de Maurice Halbwachs, ainsi qu'aux « notions conventionnelles de vérité »; elle implique des rituels et nécessite des « gardiens », enfin, à l'inverse des coutumes, elle opère une force liante qui combine émotions et morales ${ }^{40}$. Organisée autour de rituels et de conventions toujours interprétés, la tradition a de fortes dimensions connotatives qui ne sont pas aux seules mains des individus, mais constituent le collectif. Ce tissu traditionnel produit pour les individus « un horizon d'action relativement fixe ${ }^{41} \gg$. Ce rapport à l'étrangeté est encouragé par la globalisation, dans laquelle les modes de vie et les cultures sont de plus en plus mis en communication les uns avec les autres. Si l'acceptation de ces vies alternatives ne s'ensuit pas mécaniquement, il n'est en tout cas plus possible d'ignorer qu'elles existent. Pour Giddens, les addictions modernes sont donc des formes post-traditionnelles de répétition, qui permettent de « rester dans "le seul monde que l'on connaît", une façon d'éviter l'exposition à des valeurs ou des styles de vie "étrangers" ${ }^{42}$ ». De même, les rituels collectifs ne disparaissent pas dans la modernité. Certains résistent, se reformulent ou se déplacent, mais la différence majeure est qu'ils ne sont plus effectués sur le seul critère de la reproduction des valeurs antérieures, qui tirent leur autorité du fait qu'elles sont accomplies depuis plusieurs générations. La société post-traditionnelle se construit ainsi par des opportunités et des dilemmes. Les liens d'interdépendance ne sont plus ceux qui lient des valeurs, des structures, des rituels ou des conventions à des individus qui se définissent en fonction des générations antérieures, c'est-à-dire qui colonisent l'avenir par la (relative) reproduction du passé. La tradition incarne, non seulement ce qui est fait dans une société donnée, mais aussi la prescription des comportements : elle dit ce qui doit être fait. Cette prescription s'affaiblit dans la modernité sans disparaître totalement, comme le montre bien JeanClaude Kaufmann lorsqu'il évoque une posture de jugement typique de ces tensions, représentative du double langage des sociétés démocratiques : «Chacun fait ce qu'il veut $!^{43}$ » Anthony Giddens envisage donc plutôt 
la tradition par sa fonction mémorielle et collective, et non par la simple persistance du passé, comme si ce dernier planait statiquement sur le présent. Au contraire, s'est construit un dialogue entre tradition et modernité, un effort permanent d'interprétation du monde présent pour le lier au passé. La tradition opère ainsi comme un «médium de la "réalité" du passé ${ }^{44}$ », ce qui ne signifie pas que la tradition soit exclusivement tournée vers ledit passé. Il est intéressant de voir que chez Giddens, la temporalité qui lie la tradition à la modernité ne se contente pas d'un cadrage linéaire passé-présent-futur, et ce pour deux raisons. D'une part, car la « réalité » du passé dont le médium est la tradition est toujours reconstruite par le présent. Si la définition de la tradition est que le passé influence le présent, l'inverse est également vrai. D'autre part, car la tradition est également liée à l'avenir puisque la répétition de ses pratiques, dit Giddens, est « une manière d'organiser le temps futur ${ }^{45} \gg$. Les temporalités ainsi articulées se construisent les unes en fonction des autres, dans un mouvement d'allers retours incessants, et non dans une téléologie qui célèbrerait la post-tradition et sa réflexivité comme l'avènement d'individus « sur-conscients », sans angles morts ni plis sociopsychologiques car portés vers l'avenir, en opposition avec des sujets qui ne seraient que les vecteurs de la répétition des rôles sociaux. Les penseurs de la réflexivité ne sont pas des évolutionnistes manichéens.

Sitôt que les structures et les actions proposées par la tradition perdent de leur influence, le choix devient la clé de voûte de la modernité, une condition sine qua non de la vie des individus. Alain Ehrenberg a produit parmi les travaux les plus importants en la matière lorsqu' il diagnostique la « fatigue d'être soi ${ }^{46}$ » qu'induisent ces obligations de choisir. Le choix ne doit pourtant pas être compris comme une action purement individuelle. Si choisir s'appuie sur une volonté certes personnelle, celle-ci n'est pas exempte des influences collectives, socioculturelles, structurelles (pour Beck) voire esthétiques et économiques (Lasch). En retour, le choix n’a pas été absent de la tradition, mais il était largement prescrit par les structures et les traditions, ce qui ne veut pas non plus dire que les individus n'avaient pas de réflexivité à son égard. La détraditionnalisation qu'évoque Ulrich Beck ne signifie donc pas que le monde est sans traditions, mais plutôt que celles-ci sont désormais soumises à l'évaluation et à l'interrogation croissantes des individus. Pour autant, les individus individualisés ne sont pas d'absolus décisionnaires qui ne rencontreraient aucune résistance puisqu'à mesure 
que le futur cesse de ressembler au passé, « il est devenu à certains égards très menaçant ${ }^{47}$ ». C'est pourquoi les « risques » deviennent centraux et c'est la raison pour laquelle les individus essaient, à force de retours sur eux et d'interrogations des éléments en jeu, de les infléchir. Néanmoins, la labilité contemporaine fait que « de nouvelles aires d'imprévisibilité sont créées bien souvent par ces mêmes tentatives de les maîtriser ${ }^{48} \gg$. Les traditions amoureuses et familiales, pour autant qu'elles continuent d'exister, par exemple dans leur forme maritale, sont dans la modernité avancée expérimentées comme des prises de risque.

Réfutant donc les définitions paniquées qui amalgament individualisation et atomisation, Beck rappelle qu'il s'agit plutôt d'un processus qui désencastre et ré-encastre (disembedding and re-embedding, selon les termes d'Anthony Giddens qui s' inspire certainement, mais de façon libre, de Karl Polanyi) les façons de vivre de la période industrielle dans de nouveaux modes, dans lesquels les individus sont en charge de la production de leur biographie devenue réflexive. La réflexivité désigne l'interrogation continue des formes sociales, dont les politiques débordent les cadres traditionnels (de la législation, de l'État, des institutions...) pour embrasser, sous l'impulsion du tournant culturel, des formes plus diffuses. La distinction que reprend Beck des sciences politiques entre lepolitique, c'est-à-dire le régime politique et sa constitution institutionnelle (polity), la politique visant à modeler le social (policy), et lespolitiques comme processus au sein desquels sont discutés et disputés les positions et les partages de pouvoir (politics), est utile pour comprendre ces glissements de la production du sens.

\section{Féminisme et individualisme sont-ils fatalement antinomiques?}

Le processus d'individualisme ne remet pas en cause le fait que, pour citer Kaufmann, « à tout instant le collectif se construit avec l'individuel et l'individuel avec le collectif ${ }^{49} \gg$. En ce sens, les liens individuel-collectif ne sont pas anéantis mais repensés à l'aune de la responsabilité et de la liberté du sujet : « en l'espace d'une ou deux générations, la production du cadre éthique est passée d'un mode collectif et explicite à une auto-définition individuelle $^{50}$ ». « Chacun fait ce qu' il veut ! » vient supplanter « C'est bien, $c^{\prime}$ est $\mathrm{mal}^{51}$. » Ce bouleversement se fonde certes sur le respect de la liberté 
individuelle, mais il ne se limite pas à ce niveau micro et vient s'articuler avec des enjeux sociopolitiques : le respect des choix d'autrui est adossé à des enjeux démocratiques. L'《auto-définition individuelle » qu'évoque Kaufmann a permis, et a été permise par le développement de mouvements pour les droits civiques, dont le féminisme fait partie. L'individualisme n'est donc pas une utopie où la conscience individuelle promet le bonheur, et la réflexivité n'est pas un levier magique. Le féminisme lui-même n’a d'ailleurs jamais promis que l'émancipation serait heureuse puisque dès 1949, Simone de Beauvoir souligne que la liberté a pour corollaire l'anxiété. L'individualisme n'est pas non plus la mort de la tradition et des luttes collectives : au contraire, la nécessité de penser la modernisation de la modernité s'est faite en réponse aux thèses insatisfaisantes du post-modernisme pour lequel les structures deviennent caduques et le sens flottant.

L'opposition bien souvent formulée entre un individualisme qui n'agit qu'au niveau de l'individu et un féminisme forcément collectif se heurte au potentiel collectif des micro-actions ainsi qu' à la dialectique qui s'exerce entre mouvement social et interventions des agents sociaux, « compulsions et possibilités de manufacturer les obligations et engagements sociaux ${ }^{52} \gg$. Le sociologue des médias David Gauntlett résume les bouleversements amoureux de la fin du $\mathrm{xx}^{\mathrm{e}}$ siècle : augmentation du taux de divorce, libéralisation des mœurs sexuelles ou démultiplication des relations trouvent leur formalisation dans un niveau macro que sont les luttes féministes pour l'émancipation et l'égalité, produites par des insatisfactions quotidiennes, qui proviennent elles-mêmes d'une montée de la rationalités ${ }^{53}$. Comme le rappelle en effet Flora Davis, le féminisme prend racine dans une lutte plus large pour les droits civiques, les féministes s'étant largement inspirées dans les années 1960 du mouvement afro-américain Student Nonviolent Coordinating Committee ${ }^{54}$. Historiquement, les luttes collectives, militantes, agissant dans la sphère politique traditionnelle, ont profondément structuré ce que doit être le féminisme, tant et si bien que la question se pose désormais de savoir si son individualisation est une régression (le rejet de ce qui fait son cœur) voire un contresens (parler de féminisme individualiste serait aussi insensé que d'évoquer une lutte collective individuelle), ou si les deux termes peuvent être réarticulés ensemble. L'arrivée des héroïnes dans les imaginaires médiatiques (« héroïnes » au sens fort et individualiste du terme puisqu'elles sont des sujets indépendants et responsables de leurs 
actions) peut-elle être le signe d'une actualisation des idéaux égalitaristes à l'aune de la modernité, ou est-elle le piège de discours antiféministes visant à incomber aux seules femmes les responsabilités de leur oppression ? Puisque l'individualisation n'est pas contenue dans l'espace privé mais qu'elle s'exprime dans les deux sphères publique et privée qu'elle fait communiquer, « une reconnaissance non-institutionnelle du politique ${ }^{55} \gg$ s'effectue. Si féminisme et individualisme semblaient antinomiques par leur mode d'action respectivement collectif et individuel, c'est bien parce qu' ils étaient envisagés selon des conceptions du politique désormais obsolètes. À l'inverse, « envisagée de l'autre côté, [l'individualisation] peut bien signifier une lutte pour une nouvelle dimension des politiques ${ }^{56} \gg$ qui ont permis au féminisme de nouvelles possibilités d'action. La montée des subpolitics et l'émergence de la sub-revolution des femmes n'ont pu se faire qu'en collaboration réciproque avec les processus d'individualisation. La question se pose d'un renversement du rapport de causalité. Porteur de valeurs égalitaires puisque, selon la formule de François de Singly, « l'individualisme est un humanisme $e^{57} \gg$, celui-ci n'est pas, par principe, articulé à l'antiféminisme. À l'inverse, c'est plutôt l'idéologie antiféministe qui reprend à son compte le régime individualiste des sociétés contemporaines, lui faisant subir de lourdes mutations, dont le rejet de son caractère humaniste, pour remettre à la charge des seules femmes les conséquences de leur émancipation, et ce pour « laisser entendre que la cause des tensions éprouvées par les femmes n'est pas le maintien de la discrimination sexiste, mais les effets pervers du féminisme lui-même ${ }^{58} \gg$. Féminisme et antiféminisme produisent tous deux, et sont produits tous deux par des mouvances très différentes que l'on a trop souvent englobées sous le même terme d'《individualisme », et qui peut tour à tour être un régime démocratique et humaniste, en ce sens profondément articulé aux luttes pour les droits civiques (dont l'émancipation de l'individu), ou un argument trompe-l'œil pour réduire ce qui est structurel à la responsabilité individuelle. 


\section{Notes}

1 Braham, Peter, Allen, John, Lewis, Paul, Hall, Stuart, Political and Economic Forms of Modernity, Cambridge, Polity Press, 1992, p. 14.

2 Guénif-Souilamas, Nacira, Macé, Éric, Les Féministes et le garçon arabe, Paris, L'Aube, 2006, p. 27.

3 Gilroy, Paul, The Black Atlantic: Modernity and Double Consciousness, Brooklyn, Verso, 1993.

4 Gilroy, Paul, Against Race: Imagining Political Culture Beyond the Color Line, Cambridge, Harvard University Press, 2000.

5 Bourcier, Marie-Hélène, Comprendre le féminisme, Paris, Max Milo, 2012, p. 18.

6 Rosen, Ruth, « Limits of Liberalism », in Rosen, Ruth, The World Split Open: How the Modern Women's Movement Changed America, Londres, Penguin Books, 2006, p. 63-93.

7 Je renvoie à l'Antipornography Civil Rights Ordinance déposée en 1983 par Catharine MacKinnon et Andrea Dworkin et dont les propositions contenaient notamment la redéfinition de la pornographie comme atteinte aux droits civiques des femmes ainsi que la possibilité pour ces dernières d'intenter des procès aux industries. Ces ordonnances ont finalement été jugées anticonstitutionnelles par la Cour suprême, au nom du premier amendement protégeant la liberté d'expression.

8 Echols, Alice, Daring to be Bad. Radical Feminism in America. 19671975, Minneapolis, University of Minnesota Press, 1989.

9 Davis, Flora, Moving the Mountain. Women's Movement in America since 1960, Upper Saddle River, Prentice Hall, 1991, p. 138.

10 Delphy, Christine, L'Ennemi principal. Tome 1 : Économie politique du patriarcat, Paris, Syllepse, 2002, p. 36.

11 Wittig, Monique, La Pensée straight, Paris, Balland, 2001, p. 39.

12 Les femmes « doivent arborer leur étoile jaune, leur éternel sourire jour et nuit » écrit Monique Wittig (p. 40), une analogie évidemment abusive qui n'est pas sans rappeler celle de l'exploitation domestique des femmes avec l'esclavage.

13 Guénif-Souilamas, Nacira, Macé, Éric, ibid., p. 33.

14 Guénif-Souilamas, Nacira, Macé, Éric, ibid., p. 34. 
15 Mulvey, Laura, «Visual Pleasure and Narrative Cinema », Screen, 16, 3, 1975.

16 Lehman, Katherine, Those Girls. Single Women in Sixties and Seventies Popular Culture, Austin, University of Kansas, 2011, p. 159.

17 McRobbie, Angela, The Aftermath of Feminism. Gender, Culture and Social Change, Londres, Sage Publications, 2009.

18 Delphy, Christine, L'Ennemi principal. Tome 2: Penserle genre, Paris, Syllepse, 2001.

19 Macé, Éric, « Le piège de la "cause des femmes". Éléments pour un mouvement antisexiste postféministe », Cosmopolitiques, 4, 2003.

20 Spivak, Gayatri Chakravorty, Said, Edward, Guha, Ranajit, Selected Subaltern Studies, Oxford, Oxford University Press, 1988, p. 3.

21 Spivak, Gayatri Chakravorty, Said, Edward, Guha, Ranajit, ibid., p. 13.

22 Lehman, Katherine, ibid., p. 160.

23 Lehman, Katherine, ibid., p. 163.

24 McRobbie, Angela, ibid.

25 Faludi, Susan, Backlash. La guerre froide contre les femmes, Paris, Des Femmes, 1993.

26 Lehman, Katherine, Those Girls. Single Women in Sixties and Seventies Popular Culture, Austin, University of Kansas, 2011.

27 Shales, Tom, « Stereotypes in Videoland », Washington Post, 17 août 1977.

28 Cité par D'Acci, Julie, Defining Women: Television and the Case of Cagney \& Lacey, Chapel Hill, The University of North Carolina Press, 1995, p. 222.

29 Van Zoonen, Liesbet, Feminist Media Studies, Londres, Sage Publications, 1994, p. 153.

30 Latour, Bruno, « Is Re-Modernization Occurring - And If So, How to Prove It? », Theory, Culture \& Society, 20, 2, 2003, p 35-48.

31 Macé, Éric, L'après-patriarcat, Paris, Seuil, 2015.

32 Macé, Éric, ibid., p. 21.

33 Macé, Éric, ibid., p. 129.

34 Macé, Éric, ibid., p. 82.

35 Macé, Éric, ibid., p. 127. 
36 Beck, Ulrich, Reflexive Modernization: Politics, Tradition and Aesthetics in the Modern Social Order, Palo Alto, Stanford University Press, 1994, p. 13.

37 Beck, Ulrich, Bonss, Wolfgang, Lau, Christoph, « The Theory of Reflexive. Modernization Problematic, Hypotheses and Research Programme », Theory, Culture \& Society, 20, 2, 2003, p. 1-33.

38 Giddens, Anthony, « Living in a Post-Traditional Society », in Beck, Ulrich, Reflexive Modernization: Politics, Tradition and Aesthetics in the Modern Social Order, Palo Alto, Stanford University Press, 1994, p. 56.

39 Giddens, Anthony, ibid., p. 62.

40 Giddens, Anthony, ibid., p. 63.

41 Giddens, Anthony, ibid., p. 76.

42 Giddens, Anthony, ibid., p. 73.

43 Kaufmann, Jean-Claude, Corps de femmes, regards d'hommes. Sociologie des seins nus sur la plage, Paris, Pocket, 2010, p. 205.

44 Giddens, Anthony, ibid., p. 94.

45 Giddens, Anthony, ibid., p. 62.

46 Ehrenberg, Alain, La Fatigue d'être soi. Dépression et société, Paris, Odile Jacob, 2000 ; Ehrenberg, Alain, « De la névrose à la dépression. Remarques sur quelques changements de l'individualité contemporaine », Figures de la psychanalyse, 4, 2001, p. 25-41.

47 Beck, Ulrich, ibid., p. 3.

48 Beck, Ulrich, ibid., p. vii.

49 Kaufmann, Jean-Claude, ibid.

50 Kaufmann, Jean-Claude, ibid.

51 Kaufmann, Jean-Claude, ibid.

52 Beck, Ulrich, ibid., p. 20.

53 Gauntlett, David, Media, Gender, and Identity: An Introduction, Washington DC, Routledge, 2008, p. 97.

54 Le SNCC était, selon Flora Davis, l'organisation la plus décisive des années 1960. Fondée en 1960 par des étudiants afro-américains, la SNCC est née de leurs occupations des comptoirs de restauration refusant de servir les Noirs. Menée à l'origine par Ella Baker, le mouvement prônait l'empowerment des subalternes par l'abolition des hiérarchies que doit entériner la démocratie participative. 
55 Beck, Ulrich, ibid., p. 17.

56 Beck, Ulrich, ibid., p. 20.

57 Singly (de), François, L'Individualisme est un humanisme, Paris, Éditions de l'Aube, 2007.

58 Guénif-Souilamas, Nacira, Macé, Éric, ibid. 



\section{Les ambiguïtés du postféminisme}

\section{Du personnel politique à la politique individualiste}

Résumons. Pour Bonnie Dow et Angela McRobbie, l'adage de la deuxième vague féministe, «le personnel est politique », a été dévoyé en un «le politique est personnel », déplaçant les problèmes structurels rencontrés par les femmes dans l'univers privé. Le responsable principal en est le postféminisme qui dévalorise l'action collective et la politique militante au profit d'un cadre individualiste centré sur ego et d'une réduction de toute contrainte sociopolitique au choix personnel. Lespolitiques féministes se sont transformées en identités féministes, regrettent-elles, lesquelles ne prennent pas acte du social qui contraint ni du collectif qui émancipe. Angela McRobbie, dans The Aftermath of Feminism, produit pareilles récriminations lorsque, complexifiant la théorie du backlash de Susan Faludi, elle signale que les femmes (de surcroît, les jeunes femmes) sont dépolitisées et considère le féminisme comme dépassé. Primat des choix individualistes pour Dow, rejet du féminisme pour McRobbie, le postféminisme peine à trouver une définition stable mais désigne toujours ce moment de l'après-féminisme qui en trahit la dimension collective et « politique » au sens traditionnel. Il est curieux de voir que McRobbie formule ces critiques à l'égard des femmes et de leurs représentations médiatiques des années 1990, tandis que Bonnie Dow, en 1996, l'applique aux héroïnes des années 1970. Celles-ci, de Mary Richards à Dr Quinn, ne formulent en effet que très rarement des enjeux clairement féministes et ne font que laconiquement référence aux mouvements militants. Lorsque c'est le cas, 
l'hétérogénéité des courants et des pratiques est ramenée à l'étiquette inclusive et réductrice de « féminisme », au singulier. Toutefois, si la politique au sens traditionnel est exclue des séries analysées par Bonnie Dow, cela ne signifie pas que les idéologies féministes et même que les mouvements militants ne traversent pas ces représentations médiatiques, mais c'est moins par la représentation de femmes ouvertement féministes que de femmes aux prises avec des questions féministes que les séries abordent l'émancipation féminine. McRobbie ou Dow ont raison d'écrire que les représentations des années 1990 sont postféministes (au sens où le féminisme aurait accompli son travail et ne serait plus nécessaire), mais à condition d'entendre par féminisme sa définition libérale. Alors, en effet, de Mary Richards à Dr Mike, les représentations télévisuelles, lorsqu’elles s'attachent à montrer la conquête de la sphère publique par les femmes, semblent signifier que la tâche est accomplie et ce, sans qu'il ait été besoin de remettre en cause l'ensemble du système patriarcal. Une telle interprétation des représentations ignore néanmoins, probablement parce que les auteures les considèrent trop peu montrées, les manifestations du féminisme radical : les héroïnes ne sont pas toujours satisfaites de leur vie professionnelle, ni de l'agencement entre public et privé, encore moins de leur vie amoureuse. Maude défend des positions progressistes, par exemple sur l'avortement; les Drôles de Dames se réapproprient leurs corps et combattent des machos; Murphy Brown lutte pour ne pas céder aux arrangements inégalitaires qu'on lui propose. Si la télévision, c'est vrai, montre peu de personnages ouvertement féministes et encore moins de luttes féministes militantes, on ne peut pour autant considérer que le féminisme radical est complètement absent des représentations. Sa dimension collective est certes individualisée car la télévision tend précisément à incarner les problématiques sociales dans des héro(ïne)s, mais les questions que le féminisme a soulevées sous-tendent les thèmes traités : agencement du public et du privé, réappropriations des corps, renversement des rôles genrés, critique du patriarcat, anti-essentialisme, exploitation domestique sont constamment présents. Bonnie Dow et Angela McRobbie restent finalement prisonnières d'une conception exclusivement publique et militante du féminisme. Mary Richards n'a pas besoin d'ode explicite à Gloria Steinem pour que les récits de ses aventures soient porteurs de significations sur sa condition de femme active et célibataire. Un autre problème est que, défini par l'individualisme 
et le primat du choix personnel, le postféminisme est légitimé par une question centrale et passionnante de l'après-féminisme qu'est celle de la victimisation. Comment considérer que les choix individualistes ne sont pas la nouvelle expression d'un antiféminisme que les femmes produisent malgré elles ? Comment éviter de déposséder les femmes de leur capacité d'agir lorsque l'on décrit les oppressions, les normes et les contraintes qui pèsent sur elles, dans une tradition critique sur laquelle plane toujours le fantôme du concept d'aliénation ? Ce «disempowerment » est lui-même issu des diagnostics d'un féminisme critique qui étouffe les femmes sous le poids de structures patriarcales ancrées dans des dominations presque systématiques (à défaut d'être universelles). Cette systématicité se fait au-delà des gradations, alors que l'oppression se ressent moins chez les femmes blanches et économiquement aisées ${ }^{1}$, principales porte-paroles du postféminisme. La tendance victimisante de certains féminismes provient certainement de leur difficulté à adopter une démarche compréhensive et inductive, et à considérer les pratiques et les choix individuels comme des micropolitiques capables de perturber ce qui est encore trop souvent considéré comme des superstructures. Les analyses par ailleurs très fines de Bonnie Dow souffrent ainsi de ne pas prendre la pleine mesure des subversions culturelles produites par les médiacultures et les comportements individuels (« réels » et représentés).

Finalement, le problème posé par ces auteures semble plutôt être l'actualisation du féminisme radical à l'aune de la modernité avancée. L'émergence de l'individualisme réflexif et de sa nouvelle conception du sujet n'est pas opposée au féminisme radical, au contraire. Puisqu'il « désigne le refus de tout enfermement identitaire involontaire ${ }^{2} \gg$ et qu'il porte en son cœur l'émancipation, il a au contraire permis au féminisme radical de survivre à la deuxième modernité, moyennant quelques adaptations. L'affirmation qui s'est faite à la fin des années 1960 d'un sujet libre et indépendant a été permise par l'émancipation des femmes, qu'elle a encouragée en retour. L'individualisme réflexif n'a pas seulement bouleversé les valeurs holistes (que le féminisme radical a parfois du mal à abandonner sous ses formes militantes), ni la perception de l'individu et de ses possibles, il a aussi changé les rapports sociaux et notamment amoureux. En conclusion, si la politisation des relations intimes telle que l'envisageait le féminisme radical au début des années 1970 a du mal à percer dans les représentations télévisuelles des années 1980, c'est aussi parce qu'elle se heurte à 
un affaiblissement des modes relationnels d'alors par la réinvention des liens amoureux qu'a permis l'individualisme réflexif. On voit bien en effet qu'à partir des années 1970, les héroïnes peinent de plus en plus à trouver non plus un mari, mais un partenaire qui respecte leur individualité (Murphy Brown) et le rêve de la famille nucléaire se fait timide, quand il n'est pas frontalement attaqué (Roseanne). Dans le même temps, les sociétés étasunienne et européenne connaissent une croissance des divorces, indice d'une précarisation des relations amoureuses. Il est évident que les différentes formes de féminisme des années 1960 et 1970 ne peuvent survivre à de telles mutations sociales sans actualiser leurs objectifs et leurs propositions. Le défi de la réinvention de l'amour doit s'accompagner d'une réinvention des féminismes, cela sans balayer un individualisme réflexif précieux pour l'affirmation des identités et des politiques.

\section{Un féminisme aux influences matérialistes encombrantes pour l'étude des imaginaires culturels?}

Ce sont les influences matérialistes qui apparaissent poser des limites à l'analyse des productions culturelles : sont souvent convoquées les « vraies » conditions de vie des femmes, comme lorsque la féministe Susan Douglas, dans une approche mimétique qui s'est déjà montrée inappropriée à l'étude des imaginaires, regrette l'inadéquation des représentations médiatiques avec les statistiques de femmes actives. Or, ces influences matérialistes relèvent en réalité bien peu d'études empiriques à l'instar des travaux remarquables de Christine Delphy en la matière, mais trahissent plutôt une démarche prescriptive. Par exemple, Bonnie Dow a beau ne pas nier le potentiel féministe des médiacultures, elle ajoute immédiatement que « l'idéologie [féministe représentée] est forgée à l'aune des besoins de la télévision, et non des besoins des politiques féministes engagées dans le futur des femmes, peu importe leur race, classe, sexualité, ou situation ${ }^{3} \gg$ : les industries télévisuelles apparaissent comme une superstructure venant étrangler l'expression de la « réalité » des conditions matérielles des femmes, alors qu'elles devraient être le territoire de leur expression. Le « danger » ne se logerait donc pas dans l'appréciation des moments passés devant ces séries télévisées, mais dans la croyance que les idées peuvent 
remplacer les luttes matérielles, ce qui reviendrait à adopter une posture postmoderne, célébration aveugle des discours et images médiatiques. Les dernières lignes de Prime Time Feminism sont une définition quelque peu matérialiste du féminisme comme « politiques aux conséquences matérielles qui entraîne des choix difficiles, un travail lourd et un engagement dans l'action collective »- clef de voûte d'une hiérarchisation des actions politiques sur les représentations médiatiques car si « les images peuvent et ont contribué à ce combat, elles ne peuvent pas s'y substituer ${ }^{4} \gg$. C'est bien sur cette question que semble achopper la réflexion de Bonnie Dow. Le problème n'est pas celle de la substitution mais de la composition : il est essentiel d'abandonner cette vision linéaire des luttes sociales, considérant que le matériel précède l'imaginaire, pour envisager ce dernier comme une partie de la charpente. Il ne s'agit pas de penser que matérialité et imaginaire participent équitablement des politiques et encore moins de perdre la notion de causalité, mais d'une part de réintégrer les effets bien réels des imaginaires (ce qu'Edgar Morin a si bien montré) et d'autre part de prendre acte du tournant culturel. À l'inverse, lorsque Bonnie Dow plaide explicitement pour un renforcement de la distinction entre culture et politique 5 , elle montre la définition très traditionnelle qu'elle entérine de la communication médiatique, qu'elle limite à la rhétorique et à la politique classique. En rejetant la dimension politique de la culture et en balayant la dimension culturelle de la politique, Dow envisage les médias comme une fenêtre aux vitres étroites et déformantes sur les féminismes, à la manière des journaux télévisés sur les faits et les événements. Or, la priorité qu'elle confère aux discours, reléguant les pratiques en arrière-plan, pose d'autant plus problème que l'analyse porte non pas sur des propos publics « réels » mais sur des représentations imaginaires narratives. Cette axiologie nous fait retrouver une vieille distinction entre parole et pratiques alors même que c'est en ces dernières que se morcellent les idéologies : Stuart Hall a bien décrit la dispersion des rapports de classe « dans les agencements fragmentaires d'une myriade d'opinions et de volontés individuelles, de pouvoirs séparés » avant d'être réorganisés en « cohérences imaginaires ${ }^{6}$ », en vœu de consensus. Le primat des discours pose également problème parce qu'il est erroné de penser que ce que dit un personnage (ou d'ailleurs toute personne « réelle ») est un témoin fidèle de ses actes, un miroir de ses pratiques. Ce décollement entre ce que l'on dit faire et ce que l'on 
fait vraiment nous rappelle que les idéologies et les pratiques, loin d'être des systèmes parfaits, ne sont parfois cohérentes ni en elles, ni entre elles. Pratiques, idéologies et discours sont saturés de contradictions, tout particulièrement dans des médiacultures enclines au syncrétisme.

Pour comprendre cette difficulté qu'a Bonnie Dow à saisir les enjeux de l'imaginaire, il faut regarder du côté de son ancrage épistémologique. Formée de son propre aveu non pas à l'étude des médias mais à la rhétorique et à l'analyse des discours publics ${ }^{7}$, Bonnie Dow considère que la «télévision de divertissement accomplit une partie du travail culturel qui était auparavant produit par les discours publics $\gg$ et qu'il faut en ce sens analyser « le travail rhétorique de la télévision quand elle négocie les problématiques sociales pour les définir, les représenter et, in fine, offrir des visions de leurs sens et de leurs implications ${ }^{8} \gg$. Une telle étude des séries télévisées et plus largement de la télévision est, on le devine, particulièrement problématique car elle considère que les perspectives de la rhétorique peuvent être saisies presque indifféremment de l'objet étudié : elles seraient valables pour les discours publics du XIX $\mathrm{X}^{\mathrm{e}}$ et du début du $\mathrm{XX}^{\mathrm{e}}$ siècle comme pour la télévision industrielle des $\mathrm{XX}^{\mathrm{e}}$ et $\mathrm{XXI}^{\mathrm{e}}$ siècles. Enfin, envisager les séries télévisées comme des discours rhétoriques visant à persuader, c'est apposer un filtre prescriptif à l'étude des séries télévisées, dictant ce que la télévision devrait montrer (des femmes émancipées sans prix à payer) au lieu de comprendre le processus démocratique qui est à l'œuvre dans l'hétérogénéité des hégémonies et contre-hégémonies. Réintégré à l'analyse, celui-ci permet d'envisager que la tension entre standardisation et innovation pour fédérer des publics très différents leur permet précisément de dialoguer. Les recherches de Bonnie Dow souffrent ainsi de ne pas être sous-tendues par une analyse socioéconomique des chaînes de télévision et/ou des représentations médiatiques, un écueil imputable à son inscription dans le champ des communication studies anglosaxonnes qui tendent à négliger, selon Douglas Kellner, « la spécificité des textes culturels, leurs effets et leurs usages par les publics [et à] se concentrer sur une partie seulement du circuit de communication et de culture ${ }^{9} \gg$. En somme, si les tendances hégémoniques des productions télévisuelles produisent effectivement une réduction et une simplification des problématiques et des courants féministes comme le défend Bonnie Dow, ce dont ne peut se défaire une analyse des industries culturelles, ses travaux 
souffrent en retour de ne pas proposer une démarche compréhensive des médias de masse, $s^{\prime}$ inscrivant dans des logiques de production industrielles et culturelles. Une telle démarche devrait permettre de compléter, au côté pile de l'hégémonie, de la standardisation et des normes, le côté face de la contre-hégémonie, de l'innovation et des réinventions quotidiennes, trop souvent oubliées. Que la métaphore monétaire ne trompe pas : côté pile et côté face, pour autant qu' ils sont dans des processus de dialogie et de compromis, ne sont pas sur un pied d'égalité mais dans une lutte constante de définitions du monde. Pour résumer, cette difficulté à analyser les héroïnes de séries télévisées comme des incarnations idéologiques des définitions $\mathrm{du}$ monde provient d'une triple erreur : d'abord d'une démarche prescriptive, qui tend à dire ce que les médias devraient montrer plutôt que de favoriser une compréhension des tensions idéologiques; ensuite de l'insuffisante prise en considération de la dimension politique et humaniste de l'individualisme réflexif, entendu seulement comme l'arrachement du sujet au combat politique ; enfin, d'une définition anachronique du sujet, perçu comme maillon du collectif, et non comme individu autonome, capable de micropolitiques et de subversions quotidiennes.

\section{Un concept nébuleux pour une fracture générationnelle}

$\mathrm{Au}$ fond, le postféminisme est un champ plus labile que celui décrit par Bonnie Dow ou Angela McRobbie : il est avant tout un mouvement contradictoire et un concept nébuleux, marqué par une forte fracture générationnelle. Le postféminisme est envisagé tour à tour comme un backlash, comme une branche de la troisième vague ou comme étant en articulation avec le post-modernisme, le post-structuralisme et le post-colonialisme. Se confondant quelquefois, mais pas toujours, avec l'antiféminisme, le postféminisme décrit dans l'après-féminisme la libération parfois joyeuse « des chaînes idéologiques d'un mouvement féministe dépassé et sans espoir ${ }^{10} \gg$. Célébration d'un individualisme souvent consumériste (dont les garde-robes des héroïnes de Sex and the City sont de fidèles témoins), il faut rappeler que le postféminisme ne fait pas partie des mouvements politiques féministes mais a été le terme apposé par ces derniers sur un ensemble de représentations socioculturelles affleurant au milieu des années 1980. 
Dans le champ académique, l'expression apparaît en 1987 sous la plume de Deborah Rosenfelt et Judith Stacey pour désigner l'émergence d'une culture et d'une idéologie qui «incorpore, révise et dépolitise un bon nombre des questions fondamentales de la deuxième vague ${ }^{11} \gg$. Plus largement, le postféminisme tend désormais à se « cristalliser autour de questions comme la victimisation, l'autonomie et la responsabilité ${ }^{12} \gg$. Humanisme libéral qui refuse de considérer les femmes comme des proies passives et qui tente de trouver une place aux hommes, le postféminisme pose en son épicentre la question maintes fois répétée par les personnages : peut-on tout avoir, relation amoureuse et vie professionnelle épanouissante, féminité et féminisme ? Parce que les héroïnes postféministes étudient le problème de l'héritage du féminisme de la deuxième vague alors même que tous ses objectifs n'ont pas été atteints, et que ceux qui l'ont été restent fragiles, elles se sont attiré les foudres des théoriciennes féministes qui y voient une trahison des idéaux défendus durant les années 1960 et 1970. L'expression des peurs et des doutes des personnages féminins est perçue tantôt comme une réaction antiféministe aux avancées de la deuxième vague, tantôt comme une fracture générationnelle discutant les obstacles persistants.

Au cœur de cette controverse féministe, un premier courant composé notamment de Susan Faludi, Tania Modleski, Andrea Press et Angela McRobbie envisage le postféminisme comme un backlash, un antiféminisme qui renie les avancées de la deuxième vague. Pour Modleski, le postféminisme nous ramène dans un univers pré-féministe ${ }^{13}$. Andrea Press utilise le terme pour définir un esprit antiféministe dans les séries des années 1980, arguant que ce mouvement signe « un retrait des idées féministes qui dénaturalisait le rôle traditionnel des femmes dans la famille et marque à la place une ouverture toujours plus grande aux notions traditionnelles de féminité et de rôles féminins ${ }^{14}{ }^{\text {}}$. Pour Susan Faludi et Angela McRobbie, postféminisme et backlash se confondent et doivent leur succès à leur capacité à se faire passer pour ironie quand, en réalité, ils attaquent les laborieuses et fragiles victoires égalitaires de la deuxième vague. Dans cette stratégie, le rôle des médias est central car ils convainquent les femmes de la désuétude du féminisme en montrant des héroïnes malheureuses du fait de ses accomplissements (Bridget Jones, Ally McBeal...), blessure profonde que seul le grand amour pourra guérir. Le chapitre « Postfeminism and Popular Culture » d'Angela McRobbie est paradigmatique de ce 
mouvement. En complexifiant l'hypothèse du backlash de Susan Faludi, McRobbie identifie le paradoxe d'une représentation massive à partir des années 1990 de personnages principaux féminins et d'une « accumulation de réponses ambivalentes et apeurées ${ }^{15} \gg$. Elle voit dans la figure de la blonde ambitieuse (Bridget Jones, Ally McBeal, Sex and the City) l'archétype d'un nouvel antiféminisme soutenu par l'individualisme contemporain. L'hypothèse de McRobbie est celle d'une fracture générationnelle entre les femmes, à la manière de la fracture de classe irréconciliable chez Simone de Beauvoir, qui prévaut sur toute considération féministe et rend le féminisme lui-même désuet pour l'unique raison qu'il date de quelques décennies. L'« idéologie cool » se nourrit de la jeunesse et prône le présentéisme. À cela s'ajoute, pour McRobbie, une « relation non critique aux représentations sexuelles produites commercialement [qui] invoquent une hostilité à assumer des positions féministes du passé, pour adhérer à un nouveau régime de significations sexuelles basées sur le consentement féminin, l'équité, la participation et le plaisir, libre de toute politique ». Pour être libre de boire et de fumer, de faire la fête et l'amour avec qui bon leur semble, sont requis des femmes le silence public et la retenue de toute critique. Ces représentations semblent dire que les femmes réussissent, non pas grâce aux avancées féministes de la deuxième vague, mais grâce aux vertus de l'individualisme auquel elles ont enfin accès. Au diagnostic de backlash, Bonnie Dow préfère le terme de « postféminisme » pour désigner quelque chose de sensiblement différent que les regrets générationnels de McRobbie. Selon elle, dès Murphy Brown en 1988, les héroïnes postféministes ne désavouent pas les victoires du féminisme mais y portent des critiques conjointement mélioratives et péjoratives qui interrogent l'héritage de la deuxième vague. Mais, ignorant les effets structurels, le postféministe remet aux seules femmes la responsabilité des inégalités de genre persistantes, suggérant que celles-ci ne peuvent être résolues que dans les choix individuels et les subtils dosages entre privé et public.

De nombreuses critiques ont été formulées à l'égard de cette définition du postféminisme, critiques qui privilégient une approche moins nostalgique. Une frange des chercheur.e.s envisage plutôt ces représentations médiatiques comme une interrogation socioculturelle de l'héritage du féminisme. Une première erreur, en effet, est de n'imaginer le postféminisme que dans son « post », c'est-à-dire comme une réponse à, ou une 
suite imparfaite de la deuxième vague, sans prendre en considération les grandes mutations sociales qu'ont connues les sociétés occidentales ces cinquante dernières années, et dans lesquelles le féminisme lui-même a joué un rôle majeur. Or, pour Stephanie Genz et Benjamin Brabon, « la pluralité et l'“impureté” du postféminisme [ne peuvent être comprises que comme] symptomatiques d'une modernité avancée encline à la contradiction et d'un environnement socioculturel changé, caractérisé par des discursivités complexes et des interactions contextuelles ${ }^{16}{ }$. Le postféminisme, arguent-ils, n'a pas été seulement enfanté par le féminisme mais est aussi lié à d'autres mouvements comme l'individualisme libéral ou le consumérisme, auxquels on peut ajouter, angle mort de leur ouvrage, la réflexivité.

En extrayant le postféminisme de son contexte socioculturel pour ne l'envisager que comme une réponse à la deuxième vague, Faludi, Modleski, McRobbie et, dans une moindre mesure, Dow, peinent à considérer la culture populaire comme une sphère publique. Elles la soupçonnent, au contraire, de pervertir des formes supposément pures du féminisme, produisant toujours une opposition entre l'authenticité politique et la culture populaire. Rachel Moseley et Jacinda Read s'opposent à cette exaltation nostalgique de la deuxième vague qui se fait au détriment d'une compréhension des imaginaires médiatiques comme médiateurs des controverses sociales : elles plaident pour explorer les manières dont les représentations médiatiques agissent comme «l'un des territoires de production et d'exploration du féminisme ${ }^{17} \gg$. Dit autrement, il apparaît difficile de calquer mécaniquement des mouvements féministes euxmêmes hétérogènes et contradictoires sur des représentations médiatiques qui sont forcément polysémiques. Amanda Lotz souligne, à raison, qu'il est « impossible d'argumenter de façon persuasive que telles séries sont féministes ou antiféministes du fait de leur nature contradictoire et de leur usage sophistiqué d'appareils narratifs, comme de la complexité de leurs formes textuelles et du contexte de programmation dans lequel elles circulent $^{18} \gg$. On peut ajouter qu'un amalgame handicape régulièrement les réflexions sur le postféminisme : peu d'auteur.e.s distinguent en effet la représentation de la légitimation. Or, ce n'est pas parce qu'un programme télévisuel aborde un sujet qu'il le légitime. Évoquer, par exemple, les problèmes de l'articulation de la vie professionnelle avec la vie privée n'est pas forcément légitimer cette distinction patriarcale. La norme étant ce 
qui relève de l'évidence, c'est d'ailleurs déjà dénaturaliser cette opposition que de la problématiser. De telles thématiques signifient plutôt la prise en charge de problèmes caractéristiques des esprits du temps pour les discuter, de façon parfois progressiste et parfois conservatrice, dans une sphère publique médiatique dont le registre est celui des imaginaires. Donner représentation à une idéologie n’est donc pas nécessairement valoriser ladite idéologie, une affaire qui reviendra aux modalités de la représentation en question, comme on le sait bien, par exemple, à propos des représentations des personnages queer dont il ne suffit pas qu' ils soient montrés pour être acceptés, valorisés ou subversifs.

De telles critiques réintègrent conjointement les logiques de production des séries télévisées et leur participation à la sphère publique. Ainsi remises en perspective, les approches de Susan Faludi et d'Angela McRobbie témoignent de la difficulté d'une certaine frange de la deuxième vague à s'actualiser aux problématiques d'une modernité désormais bien avancée et que ladite deuxième vague a pourtant encouragée. Lorsque Faludi écrit de façon péremptoire que « tout mouvement ou philosophie qui se définit comme "post" ce qui était là avant est voué à être réactionnaire ${ }^{19}$ » ou lorsque McRobbie déplore le manque d'activisme collectif des nouvelles générations sans jamais s'interroger sur les raisons pour lesquelles ces dernières s'en détournent, elles tendent à donner raison à Benz et Brabon pour qui « les modèles et les cadres interprétatifs de l'action politique comme les entend la deuxième vague doivent être étendus pour inclure les différentes capacités d'agir et positions subjectives que les individus prennent dans la culture du $\mathrm{XXI}^{\mathrm{e}} \operatorname{siècle~}^{20} \gg$. Le postféminisme tel que l'entend Bonnie Dow est là encore plus subtil que les définitions antiféministes de Faludi, Modleski et McRobbie : le courant admet de tels anachronismes en suggérant que les femmes résistent à s'identifier comme féministes car elles n'en voient pas le rapport avec leurs conditions matérielles d'existence contemporaines : « les changements d'attitude à l'égard du féminisme ne représentent pas toujours un rejet de la libération des femmes mais plutôt un ajustement à son égard ${ }^{21} \gg$. L'enquête empirique de Pamela Aronson confirme cette perspective, montrant que si les femmes rechignent à s'identifier explicitement comme féministes, elles approuvent sans concession les linéaments égalitaires du mouvement ${ }^{22}$. Si l'hypothèse d'une fracture générationnelle est largement répandue dans ces travaux, les causes divergent : pour Benz 
et Brabon, elle est encouragée par les difficultés de la deuxième vague à s'adapter aux problématiques de la modernité avancée. Le postféminisme considèrerait le féminisme accompli parce que les héroïnes représentées peinent à l'appliquer tel quel à une époque désormais réflexive et individualiste. En ce sens, en effet, si McRobbie critique beaucoup les jeunes générations, elle peine en retour à bien vouloir tenter quelques redéfinitions et critiques du féminisme de la deuxième vague. Cela ne signifie pas pour autant que le postféminisme est antiféministe. Moseley et Read ont bien compris que les représentations de trentenaires anxieuses ne sont pas une trahison des idéaux féministes mais la confrontation de ces derniers avec de nouvelles générations : « si Ally McBeal intéresse des vingtenaires et des trentenaires pleines d'anxiété, c'est parce qu'elle dramatise avec efficacité les problèmes, les luttes et les contradictions que rencontrent ces jeunes femmes contemporaines ${ }^{23} \gg$. En proposant une lecture plus conflictualiste, Moseley et Read parviennent à éclairer d'un jour nouveau la dimension profondément générationnelle du postféminisme qui s'exprime dans ces conciliations hésitantes entre « désirs féministes et désirs féminins ${ }^{24}$ ».

\section{Le postféminisme, courant postmoderne?}

Affranchie de la définition réductrice d'une politique féministe forcément traditionnelle, collective et publique, la réflexion sur le postféminisme s'attache à comprendre les contradictions contemporaines que rencontrent les femmes dans les représentations médiatiques. La plus forte d'entre elles est la conciliation difficile du féminisme avec une féminité qui n'est plus essentielle et avec laquelle on peut désormais jouer. Puisqu'il « célèbre et comprend les modes conventionnels de féminité comme n'étant pas nécessairement en conflit avec le pouvoir féminin ${ }^{25}$ », le postféminisme repense les tensions entre féminisme et féminité. En conséquence, pour Moseley, les représentations sont postféministes lorsqu'elles tentent de réintégrer aux politiques féministes une féminité traditionnelle, sans pour autant l'exalter comme essentielle. Cette proposition, parmi les plus intéressantes, montre que les représentations font paradoxalement le pari d'un féminisme perturbant le système patriarcal et affirmant la labilité des genres sans pour autant remettre en cause la féminité. Cette approche permet 
de comprendre des héroïnes aux qualités syncrétiques comme Murphy Brown, physiquement féminines (cheveux longs et blonds, talons hauts, bijoux, foulards...) et comportementalement masculines (austères, franches, déterminées, ambitieuses...), mais il lui manque une dimension réflexive que Myra MacDonald appelle « une conscience de soi parodique ${ }^{26} »:$ les individus jouent des codes genrés qu' ils dosent à leur façon. Une héroïne comme Samantha Jones (Sex and the City) mélange par exemple une apparence très féminine avec un comportement sexuel codé masculin. Dans la mesure, néanmoins, où les personnages comme Roseanne qui inversent ces caractéristiques (comportement féminin et apparence masculine) ne sont pas qualifiés de postféministe, on voit bien la dimension conservatrice du mouvement qui refuse d'abandonner les codes féminins classiques, même s'il les teinte de post-modernisme en en révélant les artifices, comme l'a bien montré Niall Richardson à propos de Bree van de Kamp ${ }^{27}$ (Desperate Housewives). Pour ces auteur.e.s, le postféminisme n'est pas backlash parce qu'il ne dénonce pas les avancées de la deuxième vague et parce qu'il n'essentialise pas les qualités féminines ni forcément ne les exalte. En revanche, l'ironie et le flottement de sens du post-modernisme qu'il récupère lui permettent de se demander si l'on peut être féminine et féministe, sans considérer la féminité comme absolue (bon nombre d'héroïnes jugées postféministes ont des traits masculins comme Murphy Brown) ni essentielle (leur féminité est distanciée, graduelle et parfois ironique comme celle de Buffy). Cette dimension est importante car elle provient directement d'un individualisme réflexif que les théoriciennes critiques peinent à intégrer à leurs écrits. Pour saisir pleinement les représentations des années 1990 comme Buffy ou Alias, on ne peut ignorer l'exagération de leurs marques de féminité lorsqu'elles combattent en pantalon de cuir ou se griment en bombe sexuelle. Cela vaut également pour des séries plus « réalistes », dont les personnages ne sont ni super-héroïnes ni espionnes : quand Carrie Bradshaw (Sex and the City) réalise qu'elle ne peut pas acheter son appartement car elle a dépensé 40000 dollars dans de luxueuses chaussures, l'exagération du consumérisme contemporain accentue une féminité déjà bien parodiée par le défilé de mode quotidien qu'elle performe. Cette conciliation, sinon impossible en tout cas difficile, de la féminité avec le féminisme est paradigmatique de la question clé de voûte du postféminisme : peut-on tout avoir ? 
Pour analyser ces représentations, il faut s'éloigner d'une approche prescriptive articulée autour du concept de « rôles modèles ». Une telle herméneutique, hégémonique dans la critique féministe étasunienne de la télévision, est vouée à l'échec car elle est basée sur l'hypothèse d'effets directs et ne rend pas fidèlement compte de la complexité narrative. Comme le résume brillamment Amanda Lotz, les séries requièrent plutôt « un engagement critique avec la question de savoir pourquoi elles entrent en résonance avec leurs publics, plutôt que de décrier catégoriquement le potentiel féministe d'un personnage parce que sa jupe est trop courte, parce qu'elle est trop mince ou parce qu'elle admet ne pas trouver un épanouissement total dans son travail ${ }^{28} \gg$. En nous enjoignant de nous détacher de la vision antiféministe du postféminisme pour embrasser une démarche compréhensive de ce mouvement, Ann Brooks en propose une définition qui prend toute sa pertinence dans son application par Amanda Lotz. Pierre angulaire des analyses des héroïnes des années 1990, la définition est longue mais mérite d'être citée dans sa totalité : le postféminisme « se détache conceptuellement des débats sur l'égalité dans le féminisme pour s'intéresser à ceux sur la différence. Il produit fondamentalement, non pas une dépolitisation du féminisme, mais un tournant politique dans les théories et les concepts du féminisme. Le postféminisme engage un dialogue critique avec les précédents concepts et les précédentes stratégies politiques et théoriques féministes, cela du fait de son inscription dans d'autres mouvements sociaux progressistes : le postféminisme exprime ainsi l'intersection du féminisme avec le post-modernisme, le post-structuralisme et le post-colonialisme. En tant que tel, il représente un mouvement dynamique capable de contester les cadres modernistes, patriarcaux et impérialistes. Il facilite ainsi une conception plus étendue et pluraliste de l'application du féminisme, tout en répondant aux demandes des cultures marginalisées, diasporiques et colonisées d'un féminisme non-hégémonique capable de donner une voix aux féminismes locaux, indigènes et post-coloniaux ${ }^{29} \gg$. Pour Amanda Lotz, le postféminisme est la troisième sous-branche du féminisme de la troisième vague, une troisième vague attentive aux diversités des identités féminines (des problématiques de race jusqu'à la variété des sensibilités féministes), comprenant que l'oppression vécue par une femme n'est pas la même que celle vécue par une autre. Cette troisième mouvance, nouvelle voie pour le renouvellement du féminisme de la deuxième vague par son attention 
aux problématiques de choix, de diversité, d'engagement, de subalternité, d'identités de genre et de déconstruction semble vouloir lier théorie et pratique, un problème fameux des mouvements féministes depuis les visions excluantes de la deuxième vague et depuis le rejet postféministe de la théorisation. Dans cette perspective, le postféminisme est un mouvement qui prend acte de la complexification de la question de l'émancipation, du fait de la multiplication des générations et des demandes de reconnaissance.

Ces précisions faites, le qualificatif de «postféministe » appliqué, par exemple, à Sex and the City prend un tout autre sens : celui, non pas d'une trahison de la deuxième vague du fait du port régulier de jupes, mais $\mathrm{d}$ 'interrogations de l' héritage de la deuxième vague et du renouvellement du mouvement féministe en fonction des envies et des attendus très différents de ses quatre personnages principaux, plaçant la diversité des approches féministes au cœur de son récit. Amanda Lotz conclut son plaidoyer pour un postféminisme englobé dans la troisième vague en énumérant ses quatre principaux attributs : «l'exploration des diverses relations au pouvoir que les femmes occupent [;] la représentation de solutions variées et du relâchement de l'activisme [;] la déconstruction des catégories binaires de genre et de sexualité désormais vues comme flexibles et indistinctes, [enfin] l'examen par les séries de situations illustrant les luttes contemporaines qui font face aux femmes et aux féministes ${ }^{30} \gg$. Les héroïnes postféministes veulent concilier ce qui a traditionnellement été construit comme inconciliable, cela en faisant un minimum de compromis : lorsqu'un juge estime indécemment courtes les jupes qu'Ally McBeal porte au tribunal, l'avocate refuse de céder à ce qu'elle considère être une atteinte à sa liberté et s'obstine à les porter jusqu'à être placée en détention pour insulte à la Cour. Aussi Lotz propose-t-elle l'expression de « nouvelle "nouvelle femme” », en référence à la « nouvelle femme » des années 1960 et 1970, pour désigner les héroïnes postféministes des années 1990. Parce qu'elles refusent « de distinguer et de choisir entre public et privé, entre identités féministe et féminine, [entre] vie professionnelle et vie domestique ${ }^{31} \gg$, des personnages comme Ally McBeal ou Carrie Bradshaw mettent au cœur de leur construction individualiste l'envie postféministe de « tout avoir »: carrière et romance, bureau et chambre à coucher, sérieux et féminité. Mais tout avoir sans faire de compromis ne peut se faire qu'en embrassant la contradiction. Ally McBeal et Carrie Bradshaw sont névrosées, dépriment 
un peu, se bercent d'illusions, hallucinent, imaginent des mondes parallèles et, finalement, revendiquent le droit à la contradiction et à l'hétérogénéité, loin de la femme au foyer parfaite des années 1950 et de l'idéal de la working woman. Le postféminisme disqualifie justement les héroïnes modèles et privilégie des femmes incertaines et imparfaites, gaffeuses et pourtant ambitieuses, professionnelles et sérieuses. La « nouvelle "nouvelle femme" » est ainsi composée des deux grandes dimensions de la deuxième modernité : côté face, elle s'inscrit dans l'individualisme réflexif car elle est compétitive, consumériste, séduisante, ambitieuse, pleine d'esprit, déterminée et ouverte ; côté pile, elle subit l'acidité de la liquidité moderne car elle est névrosée, peu sûre d'elle, compulsive, superficielle et vit des épisodes de déprime $^{32}$. Les hérö̈nes sont déraisonnables car la réalité elle-même n’est pas unie et stable mais hétérogène et fuyante, construite par les individus qu'elle construit en retour. En ce sens, Ally McBeal a beau être perdue, elle est en fait un personnage très actif : faisant des erreurs, recommençant, s'entêtant, s'obstinant, elle ne cesse de devoir préférer telle ou telle voie, envisageant le chemin comme la véritable quête. Elle incarne l'injonction faite aux individus par la deuxième modernité de devoir choisir constamment. L'expérience individuelle prend ainsi une place toute nouvelle à partir des années 1990 , et montre son potentiel politique dans sa richesse, sa diversité et sa logique ascendante : les vies sont faites de décisions individuelles sans nier les trajectoires sociales, les inégalités des chances ou les structures : Carrie Bradshaw (Sex and the City) s'entête contre un Mr Big à la distance patriarcale ; Ally McBeal est victime de harcèlement sexuel ; Lorelai Gilmore (Gilmore Girls) fuit sa famille bourgeoise ; enfant, Sydney Bristow (Alias) a été conditionnée par son père pour devenir, comme lui, une espionne; le destin de la Tueuse a désigné Buffy bien malgré elle. Les héroïnes, aussi individualistes soient-elles, ne sont pas sans structures mais la « nouvelle "nouvelle femme" » est plus troublée que la « nouvelle femme » ne l'était car, embarquée dans les introspections de la deuxième modernité, elle pense « l'entre-deux et l'incohérence comme un territoire d'épanouissement ${ }^{33} \gg$. Pour l'hérö̈ne postféministe, le problème n'est plus la conquête de la sphère publique mais de voir les luttes émancipatrices se réfugier dans la sphère privée et notamment dans la structure du couple. Cette dernière est interrogée par le postféminisme qui ne remet pas en cause les positions comportementales du masculin, même s'il remodèle 
les masculinités en favorisant celles qui sont douces et en réservant les manifestations virilistes aux ennemis. De façon globale néanmoins, les personnages masculins semblent n'avoir que peu de responsabilités dans les inégalités persistantes et l'idéologie postféministe des héroïnes des années 1990 ne les intègre que très rarement dans les problématiques émancipatrices. Cela est bousculé à partir des années 2000, lorsque des héroïnes quadragénaires commencent à formuler des reproches féministes à leur époux et à chercher, auprès de partenaires à la masculinité plus douce, des formes de relation pure. 


\section{Notes}

1 hooks, bell, Feminist Theory: From Margin to Center, New York, South End Press, 1984.

2 Singly (de), François, L'Individualisme est un humanisme, Paris, Éditions de l'Aube, 2007, p. 14.

3 Dow, Bonnie J., Prime-Time Feminism. Television, Media Culture, and the Women's Movement Since 1970, Philadelphia, University of Pennsylvania Press, 1996, p. 214.

4 Dow, Bonnie J., ibid., p. 215.

5 « Je discute également une autre dichotomie dont je pense qu'elle devait être renforcée plutôt que dissoute : celle entre culture et politique. », Dow, Bonnie J., ibid., p. 9.

6 Hall, Stuart, «Culture, The Media and the "Ideological Effect" », in Curran, James, Gurevitch, Michael, Woollacott, Janet, Mass Communication and Society, Londres, Sage Publications, 1979, p. 339.

7 «Une grande partie de la perspective que j'adopte sur la télévision provient du fait que je n'ai pas été formée aux media studies mais aux rhetorical studies », Dow, Bonnie J., ibid., p. xiv.

8 Dow, Bonnie J., ibid., p. xv.

9 Kellner, Douglas, « Communication vs. Cultural Studies: Overcoming the Divide », Communication Theory, 5, 1995 p. 162-177.

10 Gamble, Sarah, The Routledge Companion to Feminism and Postfeminism, Routledge, 2002, p. 36.

11 Rosenfelt, Deborah, Stacey, Judith, « Second Thoughts on the Second Wave », in Hansen, Karen, Philipson, Ilene, Women, Class and the Feminist Imagination. A Socialist Feminist Reader, Philadelphia, Temple University Press, 1990.

12 Rosenfelt, Deborah, Stacey, Judith, ibid., p. 36.

13 Modleski, Tania, Feminism Without Women. Culture and Criticism in a "Postfeminist" Age, Londres, Routledge, 1991, p. 3.

14 Press, Andrea L., Women Watching Television. Gender, Class, and Generation in the American Television Experience, Philadelphia, University of Pennsylvania Press, 1991, p. 4.

15 McRobbie, Angela, ibid.

16 Genz, Stephanie, Brabon, Benjamin, Postfeminism. Cultural Texts and Theories, Edinburg, Edinburg University Press, 2009, p. 6. 
17 Moseley, Rachel, Read, Jacinda, « "Having It Ally": Popular Television (Post)Feminism », Feminist Media Studies, 2, 2002.

18 Lotz, Amanda D., Redesigning Women. Television after the Network Era, Urbana, University of Illinois Press, 2006, p. 98.

19 Faludi, Susan, Backlash. La guerre froide contre les femmes, Paris, Des Femmes, 1993, p. 15.

20 Genz, Stephanie, Brabon, Benjamin, ibid., p. 39.

21 Dow, Bonnie J., Prime-Time Feminism. Television, Media Culture, and the Women's Movement Since 1970, Philadelphia, University of Pennsylvania Press, 1996.

22 Aronson, Pamela, « Feminists or "Postfeminists"?: Young Women's Attitudes toward Feminism and Gender Relations », Gender and Society, 17, 2003, p. 903-922.

23 Moseley, Rachel, Read, Jacinda, ibid., p. 231-249.

24 Moseley, Rachel, Read, Jacinda, ibid.

25 Moseley, Rachel, « Glamorous Witchcraft. Gender and Magic in Teen Film and Television », Screen, 43, 2002, p. 403-422.

26 MacDonald, Myra, Representing Women. Myths of Femininity in the Popular Media, Londres, Hodder Education, 1995, p. 100.

27 Richardson, Niall, « As Kamp as Bree. The Politics of Camp Reconsidered by Desperate Housewives », Feminist Media Studies, 6, 2006, p. 157-174.

28 Lotz, Amanda D., ibid., p. 117.

29 Brooks, Ann, Postfeminisms. Feminism, Cultural Theory and Cultural Forms, Londres, Routledge, 1997, p. 4.

30 Lotz, Amanda, « Postfeminist Television Criticism: Rehabilitating Critical Terms and Identifying Postfeminist Attributes », Feminist Media Studies, 1, 2001, p. 105-121.

31 Genz, Stéphanie, « Singled Out: Postfeminism's "New Woman" and the Dilemma of Having It All », The Journal of Popular Culture, 43, 2010, p. 97-119.

32 Genz, Stéphanie, ibid.

33 Genz, Stéphanie, ibid. 

Un demi-siècle
d'héroïnes de séries
télévisées (1951-1997) 



\section{Les conditions d'émergence des personnages féminins: des émancipations sous contrôle}

\section{Les femmes, un nouveau marché pour les annonceurs}

Les femmes ont très tôt été un marché important pour les annonceurs du fait de leur influence sur les achats domestiques. À la fin des années 1970 néanmoins, les grands networks affrontent une chute d'audience importante qui va progressivement les amener à rediriger leurs attentes vers les femmes actives ${ }^{1}$ : non seulement le marché est rendu plus vaste et instable du fait du développement des chaînes du câble, mais en plus le déplacement des femmes vers la sphère professionnelle provoque un délaissement de la télévision en journée, y compris des soap operas pourtant phares pour le marché féminin. De surcroît, les mutations démographiques sont fortes. Les femmes baby boomers parvenues à l'âge adulte sont influencées par les valeurs féministes : elles dénotent une indépendance d'esprit et une autonomie économique plus importante que celles de leurs ainées, devenant ainsi une cible de choix pour les annonceurs ${ }^{2}$. Cette double émancipation crée un nouveau segment démographique qui est pris en considération dans les calculs de la mesure d'audience Nielsen dès 1976 : sont des « working women » les femmes qui travaillent plus de 30 heures par semaine, la catégorie allant des cols-blancs jusqu'aux ouvrières. Pour Byars et Meehan, à qui l'on doit un très convaincant article sur la question ${ }^{3}$, l'apparition progressive de la catégorie «femme active » par les annonceurs résulte de cinq variables. Après la défense des droits des femmes par la deuxième 
vague féministe à la fin des années 1960, de nombreuses mutations socioéconomiques marquent la deuxième moitié du $\mathrm{Xx}^{\mathrm{e}}$ siècle : à partir de 1975 , des récessions ont un impact fort sur les vies professionnelles des femmes de classe moyenne, qu'accompagnent en parallèle quelques évolutions industrielles et technologiques dans le milieu de la télévision. L'accès grandissant des femmes à la sphère professionnelle provoque non seulement une augmentation mécanique de leurs revenus mais surtout un contrôle exclusivement féminin de ces revenus, attisant l'intérêt des annonceurs et de la mercatique. Par ailleurs, les récessions économiques dont souffrent les États-Unis à partir des années 1970 (qu'elles soient dues aux crises énergétiques de 1973 ou 1979, au krach boursier de 1973 ou à la stagflation) entraînent un taux de chômage relativement élevé, et la présidence de Ronald Reagan arrange peu la situation pour des classes ouvrières et moyennes que la politique monétariste paupérise.

Pour tenter de préserver leur pouvoir d'achat, un grand nombre de foyers américains renonce pour partie à l'idéal victorien de la famille nucléaire. Les mères vont travailler pour apporter un deuxième salaire, bien que ce déplacement ne s'accompagne pas mécaniquement d'une redistribution des tâches ménagères : les femmes désormais actives restent très largement en charge des services domestiques, y compris des achats, et « la signification de ce phénomène ne s'est pas perdue auprès des publicitaires, des corporations médiatiques qui leur vendent l'accès aux consommateurs, ni auprès des compagnies qui mesurent la qualité et la quantité des publics attirés par les médias $^{4} \gg$. Ces publics nouvellement pris en considération par les mesures d'audience deviennent un segment démographique « de qualité ». Dans un contexte économique où le pouvoir d'achat est en baisse, les stratégies publicitaires intègrent désormais une cible moins peuplée, mais plus riche. Les publics féminins sont également la réponse à la perte de monopole que rencontrent les networks. À partir de 1977 en effet, Nielsen intègre à ses calculs les chaînes du câble et les enregistrements par magnétoscope $e^{5}$, lesquels sont nombreux pour des femmes actives qui souhaitent continuer de suivre leur soap opera quotidien. Quand « le business de la télévision devient plus large que le business de la diffusion ${ }^{6} \gg$, ces nouveaux calculs apportent deux mauvaises nouvelles aux networks : non seulement ceux-ci perdent des téléspectateurs qui préfèrent le câble, mais en plus, ces téléspectateurs sont « de qualité ». En conséquence, en période de récession, les publics qui 
ont les moyens de payer pour regarder la télévision sont potentiellement ceux qui vont consommer plus fidèlement les programmes, devenant ainsi la cible première des annonceurs. Une conséquence industrielle est que les networks développent leurs propres chaînes du câble, et « identifient l'audience "féminine" comme une clé pour sécuriser l'intégration des audiences upscale, et ce parl'hybridation de genres traditionnellement masculins allant des buddy cop shows aux news ${ }^{7} \gg$. Katherine Lehman ajoute à ce sujet que les femmes jeunes et/ou célibataires sont particulièrement visées par les annonceurs car l'on suppose qu'elles portent de nouvelles valeurs de liberté et de mobilité sans remettre profondément en cause les structures patriarcales ${ }^{8}$.

Les efforts des networks NBC, ABC et CBS pour attirer les femmes se traduisent par un renouveau des propositions mythologiques, jugées plus en accord avec ce segment émancipé. Ces nouveaux programmes se teintent d'un féminisme qui n'est plus contenu au registre comique mais qui investit dans le genre dramatique. Ainsi que le souligne Amanda Lotz, les discours plus explicitement féministes du Mary Tyler Moore Show, de Maude ou de Rhoda «marquent la tentative de toucher des femmes qui expérimentent des changements dans leurs statuts familiaux et économiques avec des histoires teintées de prise de conscience et de politique de choix de vie $^{9}$ ». Mary Beth Rabinovitz, de son côté, souligne l'hétérogénéisation du groupe féminin, tant au cœur des groupes sociaux que dans les imaginaires, dans les mesures et dans les stratégies des publicitaires qui, intégrant désormais des variables libérales, urbaines, juvéniles et raciales, complexifient l'image jusqu'ici hégémonique de « la » femme au foyer ${ }^{10}$. Le passage à ce qu'Amanda Lotz appelle « l'ère postnetwork » diversifie les imaginaires qu'ont les publicitaires, les producteurs et les diffuseurs de leurs publics, renonçant à l'idée d'un «public de masse » et préférant à la diffusion générale (broadcasting) une diffusion ciblée (narrowcasting). Durant les années 1980 et 1990, les séries proposant des héroïnes se multiplient (Cagney \& Lacey, Arabesque, Golden Girls, Designing Women, Murphy Brown, Roseanne... jusqu' à Ally McBeal et Sex and the City) tout comme les chaînes dédiées à un public féminin. L'emblématique Lifetime (dont le slogan est « Television for Women » de 1994 à 2006) est lancée en 1984 et sera suivie de la romantique WE (1997) puis d'Oxygen (2000), trois chaînes qui portent dans leur logo roses et arrondis les marques de la féminité qu'elles visent.

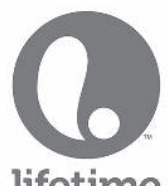

lifetime. your life your time:
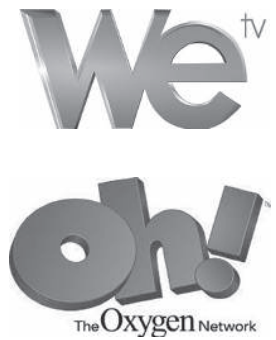


\section{Imaginaires du média et cohésion familiale}

Les idéaux de la famille, la porosité grandissante entre sphère privée et sphère publique et les imaginaires du nouveau média télévisuel participent des conditions d'émergence des héroïnes de séries télévisées. Dès ses débuts, la télévision encourage des imaginaires protoféministes car elle permet, paradoxalement, une ouverture sur le monde au moment où les femmes sont enfermées dans le foyer suburbain. De nombreux historiens ont identifié un processus homologique entre l'implantation de la télévision dans les foyers et le développement des banlieues américaines des années 1950. Alors que les politiques étatiques encouragent la construction massive des maisons suburbaines, pour « la première fois dans l'histoire, les jeunes couples trouvent moins cher d'acheter leur propre maison que de louer un appartement en ville ${ }^{11} \gg$. De telles conditions économiques bouleversent les styles de vie des jeunes étasuniens et enracinent dans l'esprit du temps l'idéologie de la propriété privée. Loin d'imaginer de façon simpliste que les fameuses «suburbs », les zones suburbaines, remplissent une fonction de refuge à l'écart des villes menaçantes, ce qui ancrerait une ferme distinction privé/public, Lynn Spigel considère que ces « jeunes couples étaient

plutôt devenus les représentants culturels de la "belle vie"12 », jouissant d'une identité publique valorisée en tant que propriétaires privés. Lorsque se développe la télévision, les discours sont à la fois dystopiques et utopiques, craignant l'éclatement de l'unité familiale ou célébrant les progrès sociaux et technologiques du média. La télévision s'inscrit directement dans l'histoire très particulière qu'entretient la culture américaine avec l'utopie d'éradication des distances, une histoire qui s'épanouit pleinement après la guerre de Sécession, avec le développement des trains, et que le télégraphe, la radio, la télévision et plus tard la conquête spatiale viennent renforcer ${ }^{13}$. La télévision promet d'ouvrir sur le monde, à l'instar du « village global » promulgué une petite décennie plus tard par Marshall McLuhan. Mais nombre de ces discours dénoncent en retour les perturbations indésirables que risque d'entraîner le média dans l'univers domestique et dans l'organisation fonctionnelle du foyer : la télévision est-elle un meuble ? Faut-il la cacher ? Les femmes peuvent-elles regarder ses programmes seules la 
journée ? Par ses représentations ou par sa simple présence, la télévision menacerait l'autorité paternelle et le charme féminin.

Ce préambule historique est essentiel pour comprendre les représentations très familiales proposées par la télévision des années 1950 jusqu'au début des années 1960. Accompagnant l'essor économique de l'aprèsguerre, les imaginaires sociaux et médiatiques se concentrent sur des familles dont les rôles genrés très marqués portent l'espoir de rétablir et de renforcer la cohésion familiale. La famille nucléaire est faite d'Étasuniens blancs de classe moyenne, « envisagés comme la clé de voûte de l'économie sociale américaine dans les années $1950^{14} \gg$, sans que les récits ne montrent systématiquement le père comme une figure intouchable. Quelques magazines se plaignent d'ailleurs, à l'époque, de ce que l'autorité du père de famille s'affaiblit : des personnages comme Ozzie Nelson, Chester A. Riley ou Ralph Kramden sont dépeints comme « des idiots primaires », regrette $T V$ Guide ${ }^{15}$ (The Adventures of $O z z i e$ and Harriet, The Life of Riley), qui n'écoutent pas suffisamment les judicieux conseils de leur femme (The Honeymooners) à qui la fin de l'épisode donne raison. Si certaines séries télévisées sont plutôt urbaines, à l'instar de I Love Lucy ou de The Honeymooners dont les récits comiques reposent sur des gags et du vaudeville, d'autres comme Father Knows Best (sic) et Leave It to Beaver substituent aux gaffes et débordements l'idéal-type de la famille nucléaire en zone suburbaine ${ }^{16}$. Produite en masse à partir des années 1940, la maison de banlieue qui peuple les imaginaires médiaculturels est truffée d'appareils électroménagers et a pour fondations des valeurs étasuniennes si fortes, comme le rêve de la prospérité, que selon l'architecte et historienne de l'urbanisme Dolores Hayden, « pour la première fois dans l'histoire, une civilisation a créé un idéal utopique basé sur la maison plutôt que sur la cité ou la nation ${ }^{17} \gg$. Cet idéal est celui de la famille victorienne aux fonctions très genrées. Pour Hayden, le contexte sociopolitique de l'après-guerre a deux priorités qui vont fusionner : d'abord, repositionner les hommes sur le marché du travail et en retirer les femmes, ensuite, construire des logements en masse. Ces imaginaires suburbains sont au cœur des séries télévisées des années 1950 jusqu'aux années 2000 (Desperate Housewives en est emblématique mais elle n'est pas la seule) et le statut de mère au foyer y est à la fois effacé et important, dominé et libéré. Dans cette économie florissante, les femmes sont simultanément rejetées de l'économie de marché et essentielles 
au consumérisme puisqu'elles en sont les principales clientes. Margaret Anderson et June Cleaver, les mères de Father Knows Best et de Leave It to Beaver, jouissent d'un pouvoir domestique concédé par le patriarcat et par l'âge d'or du capitalisme, lesquels rendent leur position dans le foyer familial indissociable de leur consumérisme.

Pensée dans l'intérêt de la famille nucléaire, l'architecture d'alors soustend une économie familiale genrée dans laquelle le père est en charge de la situation socioéconomique et la mère assure la tranquillité du foyer familial, tout en étant « à l'abri de l'hétérogénéité de classe, de race, d'ethnicité et d'âge ${ }^{18} \gg$. En ce sens, les hérö̈nes femmes au foyer, de Donna Stone (The Donna Reed Show) à Samantha Stevens (Ma Sorcière bien-aimée) en passant par Lucy Ricardo (I Love Lucy), n'ont presque jamais d'histoires à elles seules et sont plutôt montrées en train de tenir la maison en bon état, d'éduquer les enfants et de gérer les dépenses, tout cela dans l'euphorie de la cellule domestique. Elles sacrifient de bon cœur leur individualisme pour le holisme familial. Gaiement soumises, les femmes au foyer ne sont alors pas du tout désespérées : Donna Stone, Margaret Anderson ou June Cleaver sont de parfaites épouses et mères de famille qui se sacrifient pour la cohésion familiale. Leur rôle est celui de la lieutenante d'une maisonnée in fine régie par le colonel patriarche, et c'est bien sûr l'idéal (et idéal-type) de la famille étasunienne de banlieue qui se joue au lendemain de la seconde guerre mondiale. Contrairement à la France où le monopole étatique voit en la télévision un outil pour ressouder la nation d'après-guerre, les séries télévisées étasuniennes vantent le bonheur de la famille, de la propriété privée, de l'architecture domestique, de l'économie capitaliste et de son régime consumériste.

\section{Une confusion de l'amour conjugal et de l'amour familial}

Les héroïnes familiales, prises entre les convalescences d'après-guerre et les bourgeonnements de la révolution sexuelle, sont clairement influencées par les lois romantiques : les époux se sont choisis de façon exclusive et créent une histoire partagée dont les codes sont largement dictés par des valeurs comme l'idéalisation, la transcendance et la perte de soi, la fusion des âmes, la validation de l'identité par autrui. Toutefois, l'amour porté à l'époux 
se confond très vite avec l'amour porté à la famille. Donna Stone apparaît comme une femme idéale par sa capacité à se transcender dans le care et l'amour familial, ce dont témoignent les bienveillants sourires qu'elle distribue à longueur d'épisodes. Dans ces sitcoms familiales et contrairement à ce que décrit Anthony Giddens, le romantisme conjugal ne se distingue donc aucunement de l'organisation familiale : les représentations tendent au contraire à fusionner les mythes romantiques qui font alors autorité dans les imaginaires socioculturels avec les mythes familialistes d'une société étasunienne encore marquée par la deuxième guerre mondiale et que l'instabilité de la guerre froide rend anxieuse. « Siège d'énormes investissements psycho-affectifs et de la micro-économie personnelle ${ }^{19}$ », l'aménagement de la maison reflète l'ordre social genré et l'inscription dans deux puissantes idéologies d'après-guerre que sont la propriété privée et le travail, ce dernier étant tout particulièrement présent dans Ma Sorcière bien-aimée où les pouvoirs de Samantha sont considérés comme une tricherie par son mari mortel. Quand elle allume un feu du bout du nez, Jean-Pierre lui demande si « ça [la] fait se sentir coupable de savoir qu'en ce moment même, les Boys Scouts d'Amérique essaient de faire la même chose avec des bouts de bois et des cailloux ${ }^{20} \gg$. Culpabilisant Samantha, l'empêchant même d'utiliser ses pouvoirs, c'est tout un idéal méritocratique et travailliste qui se déploie dans ces banlieues américaines et qui vient sous-tendre la fonction, la place et le comportement attendu de ces héroïnes. Pourtant, cette distribution gen rée des manières d'aimer n'est pas sans rencontrer de résistances. Donna Stone exige la reconnaissance de son travail domestique, Lucy Ricardo fugue du foyer pour devenir comédienne, Samantha pratique sa magie en ignorant allègrement les récriminations de son époux.

En somme, les héroïnes ont profité de l'individualisme bourgeois pour s'émanciper du holisme traditionnel et construire une famille nucléaire qui promet la joie quotidienne tout en souhaitant, à leur échelle, profiter de quelques péripéties évasionnelles (c'est le cas de Lucy Ricardo dont le rêve de devenir comédienne est aussi celui de s'échapper du foyer domestique). Dans le même temps, portée par le syncrétisme des productions fictionnelles de masse, cette croyance se déboîte et laisse apparaître de fortes insatisfactions dont les causes sont explicitement l'organisation patriarcale contre laquelle les héroïnes se dressent de plus en plus systématiquement (son mari a beau, chaque épisode, dire non à Lucy, elle n'abandonne jamais). 
L'idéologie du bonheur se propage donc à la télévision au milieu des années 1950, moyennant quelques ajustements vis-à-vis de son alter ego cinématographique. Tandis que le cinéma porte à l'écran des amours romantiques ou des héros individuels, la télévision semble retranscrire le bonheur dans le cadre imaginé de sa réception. Les industries télévisuelles valorisent des héroïnes afin de s'adresser à un public majoritairement féminin, mais celles-ci voient le plus souvent leur individualisme écrasé par leur époux, leurs parents, leurs enfants, leurs tâches domestiques. C'est un curieux équilibre, donc, que propose la fiction télévisée des décennies 1950-1960. Ces «semi-hérö̈nes », à défaut d'être des sujets indépendants, sont bien souvent décisionnaires et les époux qui supposément décident et ordonnent sont en fait rarement suivis docilement. La stabilité des rôles genrés est omniprésente dans les structurations des personnages mais, dans les récits, elle se révèle fragile car subordonnée aux consentements de chacun et surtout de chacune. L'amour tient une place charnière puisque bien souvent, c'est lui qui légitime les abandons individuels : les épouses acceptent de tenir la maison et d'élever les enfants au nom de l'affection qu'elle leur porte, et plus encore au nom de leur amour d'aimer. À postériori, les cadres familialistes de ces séries apparaissent comme le dernier souffle médiaculturel de l'économie patriarcale telle que la concevait la première modernité. Des élans démocratiques naissent de l'expression des frustrations et des rêves.

\section{Quand les femmes aiment aimer}

« Agents secrets de la modernité » selon la belle formule d'Edgar Morin ${ }^{21}$, les femmes sont les premières à avoir développé les compétences émotionnelles qui se propageront à l'ensemble de la société par le processus de ce que d'autres auteurs ont appelé la réflexivité, mais elles en sont également le «sous-prolétariat émotionnel » pour Anthony Giddens puisqu'elles travaillent, sans reconnaissance publique, au bien-être des membres de la famille. En charge de la famille et du foyer, les héroïnes des séries télévisées voient leurs récits ancrés dans des thématiques interpersonnelles et, qu'elles excellent ou gaffent dans la gestion émotionnelle de la maison, la dimension sentimentale de leurs tâches domestiques est toujours valorisée. Le sacrifice féminin apporte aux personnages compliments et relation privilégiée aux enfants que l'on peut analyser comme autant d'avantages concédés aux 
subalternes par la visée patriarcale, rémunérations symboliques d'un travail exclu du marché de la reconnaissance. Les féministes matérialistes ont très tôt identifié ces processus, à l'instar du privilège parental ${ }^{22}$ dans lequel l'attention que les femmes portent aux enfants provoque logiquement une relation très forte entre eux qu'encourage l'autorité en retour naturalisée et distante du patriarche ${ }^{23}$. La réhabilitation du travail des femmes au foyer ainsi représentées ne concerne pas le travail ménager mais le labeur éducatif, relationnel et émotionnel et agit comme une légitimation des manières spécifiquement féminines d'aimer, une mise en lumière des bienfaisances des politiques privées du care. Jamais les hérö̈nes ne demandent à leur enfant de remettre à plus tard une requête, comme bénies par tant de sollicitations, et rarement reprochent-elles à leur époux de ne pas être disponible. Le travail domestique est pour Samantha une preuve d'amour. Joie du quotidien, preuve de l'affection portée à la famille, source même d'épanouissement personnel, il signifie que « l'on fait quelque chose pour quelqu'un que l'on aime $^{24} \gg$ et sa difficulté est exaltée comme l'authenticité de l'engagement personnel dans la structure familiale. Il faut souligner au passage que ce sacrifice qui n'en est pas un n'a plus rien de naturel car, enregistrant des subversions beauvoiriennes, les coulisses de ces ethos révèlent leur construction sociale : Samantha n'a aucun talent domestique, en particulier culinaire, et Lucy cumule les bourdes domestiques.

On doit à Carol Gilligan les réflexions liminaires sur « l'existence d'une voix morale différente, basée non pas sur les critères de la loi et de l'impartialité comme c'est le cas pour l'éthique de la justice, mais sur des critères relationnels et contextuels ${ }^{25} \gg$. Les manières d'aimer varient selon les socialisations genrées, aux femmes revenant des méthodes douces et dévouées, fondées sur l'oblativité et le sacrifice personnel qui sont articulées aux rôles genrés de la famille nucléaire. Les conseils toujours judicieux de Donna, les catastrophes domestiques lorsque Ricky gère le foyer ou la douceur bienveillante de Samantha, en somme les différentes gentleness ${ }^{26}$ des femmes, sont autant de signes de cette intelligence émotionnelle au « savoir-faire discret ${ }^{27}$ » et dont l'hégémonie est matériellement dépendante - ce que souligne une amie de Donna qui l'encourage à laisser son mari et sa fille seuls pendant une semaine : «Alex va réaliser que rester seul est bien plus dur qu'il ne l'imagine [et] c'est exactement pour ça que tu devrais partir : c'est la seule façon pour un homme de réaliser à quel point 
il est dépendant de son épouse ${ }^{28}$. $\gg$ L'éthique du care se montre pratique, expérientielle et délicate en même temps qu'elle révèle sa participation au fonctionnement antidémocratique de la famille nucléaire, qui s'exprime notamment dans l'inégale distribution de la parole légitime : les pères et enfants monopolisent les prises de parole tandis que les femmes restent dans l'écoute sauf à de rares moments où leur capital de care est suffisant pour qu'elles fassent preuve d'ingérence : « quand cesseras-tu donc de te mêler de mes affaires ? » demande Jean-Pierre, « quand tu auras appris à mieux les gérer », lui répond Samantha ${ }^{29}$. Le care n'a pas encore revêtu toutes les politiques démocratiques dont il peut être porteur («l'éthique du care, nous dit Gilligan, en tant qu'elle cultive la voix et l'écoute, est bien l'éthique de la démocratie $\left.{ }^{30} \gg\right)$ et se limite dans ces représentations à une revalorisation sans déconstruction des manières féminines d'aimer, sorte d'essentialisme stratégique.

\section{Biopolitiques des femmes au foyer}

Ce travail domestique vient s'inscrire jusque dans leurs biopolitiques. Les jupons aériens soulignent leurs tailles et accroissent leur grâce, les ports altiers et les brushings immobiles les rendent sculpturales, tandis que le respect des bienséances proxémiques et la mesure des kinésies sont autant de disciplines corporelles, simulations de la perfection féminine. Frôlant la mascarade lorsqu'elles accomplissent les tâches ménagères perchées sur talons hauts et la taille serrée d'un épais ruban, devenant burlesques lorsqu'apeurées de ne plus être regardées par leur époux, elles servent le petit-déjeuner en robe de soirée, les femmes au foyer révèlent des corps contraints par l'injonction contradictoire de l'élégance féminine et de l'ouvrage domestique. Les corps dévoilent leurs biopolitiques, leur marquage des politiques patriarcales. Territoire peu problématisé et qui ne sortira franchement de son invisibilisation qu'au milieu des années 1960 de concert avec la libéralisation des mœurs, le corps est encore un allantde-soi que les héroïnes formulent peu comme un lieu d'exercice du pouvoir patriarcal et qui peine en conséquence à s'imprégner des technologies de soi subjectivantes, comme le font pourtant les biopolitiques au fil de l'œuvre foucaldienne ${ }^{31}$. L'anatomo-politique du corps domestique finalement domestique le corps par un pouvoir patriarcal qui le recroqueville même 
jusqu'à l'infantilisation. Menaçante, la sexualité des femmes est contenue à leur intouchable et souveraine tendresse que cristallisent quelques baisers doux, une morale assumée parfois par les productrices et actrices elles-mêmes, comme Donna Reed heureuse d'avoir « prouvé que le public veut vraiment voir une femme saine et pas une gamine, pas une névrosée, pas une femme $\operatorname{sexy}^{32} \gg$. Ce n'est d'ailleurs pas un hasard si Donna Stone est l'épouse la plus chaste de notre corpus, résistant même aux tendances métaphoriques de I Love Lucy et Ma Sorcière bien-aimée qui encanaillent les personnages, à la façon de Samantha qui, dès le pilote, fait « éjaculer une flamme du briquet cassé de son époux et bouger le cendrier [pour] qu'il puisse y effleurer les cendres de sa cigarette ${ }^{33} \gg$, ou de son éloquent refus de répondre lorsqu'il veut savoir si elle est déjà allée « sur la lune » avec un autre homme que lui : «Jean-Pierre, tu ne veux pas tout savoir de moi, n'est-ce pas? Je veux dire, il y a certaines choses qu'une femme ne devrait pas dire à son mari. Le fait d'être allée ou non sur la lune en fait partie ${ }^{34}$. » Ces contraintes biopolitiques contrastent évidemment avec les libertés masculines : Ricky Ricardo, Alex Stone ou Jean-Pierre Stevens préservent encore largement leur masculinité traditionnelle, que seule parvient à tourner en dérision la belle-mère sorcière de Samantha en transformant régulièrement Jean-Pierre en oie, meuble, plante... ou en lui donnant les symptômes de son épouse enceinte lorsqu'il qualifie cette dernière de douillette. Si Foucault opère un passage des biopolitiques (imposées aux individus par des techniques disciplinaires) au teknê tou biou (qui externalise l'intériorité construite par un travail sur soi), les héroïnes restent dans le premier mouvement, celui de la toupie qui « tourne sur soi à la sollicitation et sous l'impulsion d'un mouvement extérieur » tandis que les époux traditionnels sont largement actifs et réflexifs quant à leur masculinité, à l'instar d'Alex Stone qui se laisse pousser la moustache pour réaffirmer la distinction de genre, interprétant les objections de son épouse comme un signe d'infériorité : «Tu ne serais pas jalouse ? Porter la moustache est impossible pour une femme ${ }^{35}$. » L'opposition est franche et les contestations timides entre une « extériorité intériorisée » féminine et une « intériorité extériorisée » masculine ${ }^{36}$.

En plus de leurs uniformes maternels, les femmes au foyer flirtent, volontairement ou non, avec l'infantilisation et l'érotisation. Lucy Ricardo en est la plus représentative qui, à longueur de temps, se déguise et transforme 

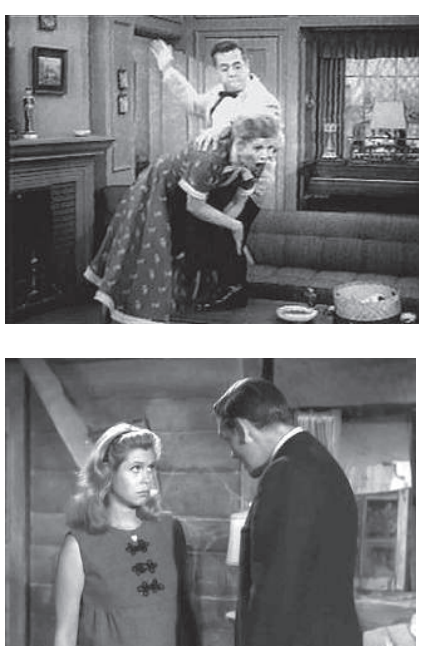

la maison tantôt en théâtre, tantôt en cour de récréation. Les caprices, pleurnicheries, clowneries et autres provocations de Lucy à l'égard de son époux l'amènent sur les genoux de ce dernier pour récolter, non pas une accidentelle correction, mais de multiples fessées tout au long de la série : « si tu te comportes comme une enfant, je vais devoir te traiter comme telle $!^{37} \gg$, prévient Ricky lorsque sa femme refuse de lui obéir. En même temps, la récurrence de ces scènes amène à se demander si elles ne permettent pas, dans une télévision très chaste où les personnages dorment dans des lits jumeaux et s'embrassent timidement, d'insérer quelques éléments sexuels.

La position dans laquelle se trouve Lucy quand Ricky la corrige accentue visuellement la disponibilité corporelle de son épouse. Dans I Love Lucy comme dans Ma Sorcière bien-aimée, les bêtises et révoltes féminines sont tolérées jusqu'à ces moments où les époux rejoignent la distribution classique du pouvoir. Alors la blondeur, les joues rosées par le blush, les jupons aux effets amincissants, tous ces codes d'élégance agissent comme signes de subordination et rappellent les effets du biopouvoir patriarcal. La soumission de l'épouse est traduite jusque dans sa corporalité, une soumission qui peut être forcée comme dans le cas de la fessée à laquelle Lucy essaie d'échapper, ou intégrée à leur ethos quand Lucy ou Samantha tentent de soutirer la compassion de leur époux par d'attendrissantes moues enfantines.

\section{Notes}

1 D'Acci, Julie, Defining Women: Television and the Case of Cagney \& Lacey, Chapel Hill, The University of North Carolina Press, 1995.

2 Haralovich, Mary Beth, Rabinovitz, Lauren, Television, History, and American Culture. Feminist Critical Essays, Durham, Duke University Press, 1999, p. 145.

3 Byars, Jackie, Meehan, Eileen, « Once in a Lifetime: Constructing “The Working Woman" through Cable Narrowcasting », Camera Obscura, 33-34, 1994-1995, p. 13-41. 
4 Byars, Jackie, Meehan, Eileen, ibid.

5 Il faut noter que si l'institut Nielsen pouvait savoir quel programme était enregistré, il ne pouvait évidemment pas savoir si ces programmes étaient ensuite regardés. L'entreprise de mesure d'audience décida pourtant de considérer que l'enregistrement équivalait au visionnage.

6 Byars, Jackie, Meehan, Eileen, ibid.

7 Byars, Jackie, Meehan, Eileen, ibid.

8 Lehman, Katherine, Those Girls. Single Women in Sixties and Seventies Popular Culture, Lawrence, University of Kansas, 2011, p. 124-128.

9 Lotz, Amanda, « Postfeminist Television Criticism: Rehabilitating Critical Terms and Identifying Postfeminist Attributes », Feminist Media Studies, 1, 2001, p. 105-121.

10 Haralovich, Mary Beth, Rabinovitz, Lauren, Television, History, and American Culture. Feminist Critical Essays, Durham, Duke University Press, 1999, p. 145-146.

11 Spigel, Lynn, Make Room for TV. Television and the Family Ideal in Postwar America, Chicago, University of Chicago Press, 1992, p. 5.

12 Spigel, Lynn, ibid., p. 6.

13 Spigel, Lynn, ibid., p. 7.

14 Spigel, Lynn, ibid., p. 113.

15 TV Guide, cité par Spigel, Lynn, ibid., p. 28.

16 Haralovich, Mary Beth, « Sitcoms and Suburbs: Positioning the 1950s Homemaker », in Spigel, Lynn, Mann, Denise, Private Screenings. Television and the Female Consumer, Minneapolis, University of Minnesota Press, 1992, p. 115.

17 Hayden, Dolores, Redesigning the American Dream. The Future of Housing, Work, and Family Life, W.W. Norton \& Company, 2002, p. 34.

18 Haralovich, Mary Beth, ibid., p. 137.

19 Morin, Edgar, L'Esprit du temps, Paris, Armand Colin, 2010, p. 197.

20 Ma Sorcière bien-aimée, saison 2, épisode 16.

21 Morin, Edgar, Commune en France, la métamorphose de Plozévet, Paris, Fayard, 1967, p. 260.

22 Delphy, Christine, L'Ennemi principal. Tome 2: Penser le genre, Paris, Syllepse, 2001.

23 On en trouve un écho médiaculturel qui vient sous-tendre les luttes des uns et des autres pour obtenir les marques de reconnaissance de leur 
fonction : après un discours public de leur fille vantant les sacrifices de sa mère, son père Alex est vexé (l'épisode s'intitule d'ailleurs « Male Ego ») que sa fille soit touchée par les attentions domestiques maternelles qu'il juge faciles et qu'il réduit à la préparation d'un lait chaud.

24 Ma Sorcière bien-aimée, saison 6, épisode 26.

25 Molinier, Pascale, «Formaliser et politiser les récits du care », Interrogations, 11, 2010.

26 Molinier, Pascale, « Au-delà de la féminité et du maternel, le travail du care $\gg$, L'Esprit du temps, 58, 2010, p. 161-174.

27 Molinier, Pascale, ibid.

28 The Donna Reed Show, saison 2, épisode 30.

29 Ma Sorcière bien-aimée, saison 2, épisode 28.

30 Gilligan, Carol, « Le care, éthique féminine ou éthique féministe ? », Multitudes, 37-38, 2009, p. 76-78.

31 Andrieu, Bernard, « La fin de la biopolitique chez Michel Foucault : le troisième déplacement $\gg$, Le Portique, 13-14, 2004. Disponible en ligne : http://leportique.revues.org/627, consultéle 14 septembre 2014.

32 «Donna Reed, oscar winner and TV star, is dead at 64 », New York Times, 15 juillet 1986, référence faite à une interview du New York Times de 1964.

33 Douglas, Susan J., Where the Girls Are: Growing Up Female with the Mass Media, New York, Three Rivers Press, 1995, p. 129.

34 Ma Sorcière bien-aimée, saison 3, épisode 17.

35 The Donna Reed Show, saison 3, épisode 37.

36 Andrieu, Bernard, « La fin de la biopolitique chez Michel Foucault : le troisième déplacement », Le Portique, 13-14, 2004. Disponible en ligne : http://leportique.revues.org/627, consulté le 14 septembre 2014.

37 I Love Lucy, saison 1, épisode 15. On peut aussi citer de la saison 3 les épisodes 1 et 19. 


\section{Retour sur les premières héroïnes, suffragettes de l'ombre}

\section{L'intercompréhension} comme remède à l'insatisfaction

Que ce soit pour en dénoncer l'ennuyeuse répétition ou pour en montrer les difficultés, les sitcoms familiales attachent une grande part de leurs représentations aux tâches ménagères accomplies par les femmes. Certaines scènes s'attardent sur les travaux des femmes au foyer, montrant avec moults détails la préparation d'un gâteau ou présentant Samantha user d'une vitesse surnaturelle pour ranger la maison, tandis que les moments de relaxation sont très largement absents des représentations et que ceux de divertissement sont conditionnés à la collectivité. Les femmes au foyer jouissent d'une sociabilité féminine, se réunissant pour jouer aux cartes, mais ne sont jamais montrées, par exemple, seules devant la télévision : dans la méritocratie des années 1950, les femmes ne sont pas oisives. L'ensemble de ces représentations domestiques définit le travail comme laborieux et empreint de tendresse maternelle, ce qu'appuient les musiques qui accompagnent la mise en scène. Les mélodies sont enjouées et les femmes souriantes qui voient dans le gâteau cuit à la perfection la fidèle retranscription de leur amour. La sphère domestique est truffée d'objets qui se chargent de l'affection de la mère, d'une multitude de non-humains agissant comme médiations de l'amour. Ces transferts silencieux sur le travail domestique redoublent non seulement les formes d'ingratitude ressenties par les femmes lorsqu'à l'instar de Donna Stone, leur époux préfère le gâteau industriel à celui fait-maison, mais aussi le sentiment d'un échec amoureux qu'elles peuvent difficilement formuler. L'inversion des rôles entre alors 
comme moyen d'atteindre l'intercompréhension. Lorsque, pour signifier son émotion de ne pas être reconnue, Donna Stone disparaît du foyer familial, les membres de la famille qui restent en charge du foyer opèrent catastrophe sur catastrophe. Pour résoudre le problème de la vaisselle s'entassant dans l'évier alors que son épouse est sur le point de rentrer, Alex Stone met sur pied une méthode qu'il qualifie avec masculinisme de « scientifique » mais qui traduit surtout son incompétence domestique : la stocker dans les placards pour la nettoyer en cachette tard le soir. De la même manière, dans I Love Lucy, lorsque Ricky veut prendre soin de son épouse enceinte, ses insuffisances créent encore plus de travail à Lucy, obligée de repasser derrière lui. Cette inversion est une idée récurrente des sitcoms familiales car elle permet de dénaturaliser la supposée facilité du labeur domestique tout en plaidant pour la continuation des rôles genrés tels qu'ils sont traditionnellement répartis. Les modalités de résolution peuvent en effet varier (la bienveillante Donna laisse croire à Alex qu'elle n'a rien vu de ses dissimulations alors que la turbulente Lucy pointe du doigt l'incompétence de Ricky), mais la morale finale est la même partout : les catastrophes domestiques signalent l'impossible mise à niveau des hommes au travail privé, et inversement des femmes au travail public, tant et si bien que désormais que chacun a appris son métier, mieux vaut ne pas toucher à cet ordre qui finalement fonctionne bien.

Toutefois, s'arrêter à la constatation d'une organisation rétablie sans réformes internes serait une erreur. Le retour à l'ordre ne serait pas possible si n'était développée au cours de ces échanges une intercompréhension, dont il faut souligner qu'elle est surtout celle des époux envers les épouses (ces dernières n'ayant jamais osé remettre en cause la vacuité du travail masculin). Des prototypes thérapeutiques affleurent dans les imaginaires médiatiques des années 1950 et encouragent, par l'expérience et l'apprentissage, à abandonner sa vision du monde pour embrasser le point de vue de l'autre. L'intercompréhension agit comme levier de reconnaissance dans une organisation maritale peu sujette à s'adapter aux envies individuelles : dans les lignes dessinées par la structure du mariage que les épouses ne parviennent pas à repousser, la gratitude de l'époux et des enfants agit au mieux comme rémunération symbolique, au pire comme lot de consolation, de sorte que leur insatisfaction est soluble dans la reconnaissance de leur époux et non dans la refonte en profondeur du système conjugal patriarcal et de 
sa division des tâches. La notion de respect est bien entendu centrale dans ces péripéties. Dans ces sitcoms familiales, entre l'après-guerre et l'émancipation féminine, le mariage de la première modernité est déterminé encore en partie sur le modèle du partenariat économique de la tradition, donc sur le respect du travail : respecter le labeur et ses conséquences, c'est respecter l'individu. L'on est bien loin de la considération que demandent les respectifs « projets réflexifs du soi ${ }^{1}$ » de la deuxième modernité où les insatisfactions émotionnelles ne s'effacent pas devant le couple souverain. Cependant, l'intercompréhension et le respect ne sont pas de simples dispositions d'esprit, des psychologies facilement enclenchées dès lors que l'un quitte son point de vue pour entendre l'autre. Les rapports sociologiques de genre, autrement dit les rapports de pouvoir genrés, construisent la division du travail et son économie domestique (et non l'inverse), ce qui signifie que les intérêts de genres se retrouvent dans ces dernières. Ricky rentrant du travail agit comme une tornade dans l'appartement méticuleusement rangé par Lucy, qui se plaint du manque de soin de son époux. Celui-ci argumente : « tu ne peux pas me demander de vivre comme dans un musée : la maison d'un homme est son château ! » , arguant implicitement que le travail domestique de Lucy doit précisément servir à ce que Ricky n'en fasse strictement aucun. Sous les airs complémentaires de la division genrée du travail tant défendus par les courants essentialistes et fonctionnalistes, les intérêts se révèlent intrinsèquement contradictoires puisque le travail et la relaxation de l'un viennent saper ceux de l'autre. L'appartement, lieu de travail pour les unes et antre de relaxation pour les autres, est tiraillé entre l'idéal petit-bourgeois de l'organisation que les femmes ont à charge et le laisser-aller d'époux en quête de coulisses après une journée de travail à l'extérieur. L'aspect éphémère du travail ménager révélé par les représentations ajoute à l'ingratitude ressentie par les héroïnes. Suffragettes du privé, leur résistance au modèle patriarcal porte ainsi, non pas tant sur les codes juridiques ou organisationnels (comme l'accès à la sphère publique), que sur les agencements culturels et psychologiques (comme le respect et la reconnaissance). Dans ces insurrections, les héroïnes éclairent l'approche fonctionnelle genrée de l'ordre familial ainsi que l'arbitraire avec lequel sont définies et valorisées ces tâches, au profit d'une vision plus culturaliste de cet ordre. D'un côté, quand Ricky sermonne Lucy sur les tâches maternelles qu'elle doit accomplir seule car lui s'occupe d'être le soutien 
financier de la famille, elle dénaturalise cette distribution en l'assimilant à un jeu inégalitaire dont elle réclame l'inversion : «Tu ne m’avais pas prévenue des règles avant que le jeu commence ! La prochaine fois qu'on a un bébé, c'est moi qui fais le père $!^{3}{ }^{\gg}$

Sans cesse infériorisées mais sans intérioriser, les hérö̈nes ne renoncent pas et, pour détourner les interdits, mobilisent les compétences sentimentales que leur assignation à la sphère privée leur a permis de développer : pour assouvir leurs envies et besoins sans que leur mari soit au courant, les héroïnes déploient des compétences du secret et de la dissimulation : Samantha passe sous silence ses dérapages magiques, Lucy se déguise et fugue pour passer des auditions, Donna manigance dans le dos de son époux. La cachette, le secret, la manipulation apparaissent alors comme autant de manières de remettre en cause l'autorité du mari, sans pour autant menacer l'ensemble du système patriarcal. Comme le souligne Jean-Claude Kaufmann, leur « arme principale est une culture de la tendresse [puisque], tenues à l'écart des sphères publiques, il s'agissait pour elles d'un des seuls instruments à leur disposition pour élargir leur influence, en investissant le nouveau domaine conjugal qui restait à imaginer et à construire ${ }^{4}$ ». Lorsque leur capacité d'agir est ainsi sapée par l'autorité patriarcale, les femmes retournent à leur avantage la confidentialité de leurs talents et la discrétion de leur champ d'action qu'est la sphère privée en agissant sur le registre du secret voire de la manipulation (ce qui sera exacerbé quelques décennies plus tard dans Desperate Housewives). L'agir est stratégique mais l'intelligence est émotionnelle et non technique : l'obstacle, lui, est invariablement abordé par son angle interpersonnel. Même lorsque le problème rencontré est aussi matériel que le budget bancal du foyer, Lucy ne tente pas une résolution par le calcul d'une meilleure gestion mais participe en cachette à un jeu radiophonique 5 . De leurs modalités d'action jusque dans la résolution, les héroïnes mobilisent réseaux sociaux, famille, amis ou collègues dont elles soutirent des comportements et des services qui leur sont utiles. Priées de formuler un choix entre privé et public, entre rêve américain de la famille et rêve professionnel, les héroïnes abandonnent leurs projets et vont retrouver leur place. Inlassablement, cet ordre rétabli se clôt sur un baiser amoureux qui n'est pas sans rappeler celui des happy ends hollywoodiens et qui peut conjointement être vu comme la récompense des femmes pour leur docilité, ou bien, puisque le téléspectateur fidèle sait 
que ladite docilité ne durera que jusqu'au prochain épisode, comme une ponctuation, une sorte de point-virgule amoureux qui permet au couple de réaffirmer son amour entre deux péripéties, en opposition au point final du happy end cinématographique.

\section{Love Lucy, héroïne burlesque et premiers assauts émancipateurs}

Les aspirations émancipatrices de Lucy menaçaient la tranquillité du mariage et ses diverses erreurs domestiques révélaient quelques dimensions beauvoiriennes que l'amour venait invariablement sauver. Lorsque sont en effet données à voir les catastrophes ménagères et culinaires que Lucy offre à son époux, il apparaît évident que la clé de leur mariage n’est pas l'idéal petit-bourgeois du foyer bien tenu mais bien leur affection mutuelle, tant et si bien que Lucille Ball décrit dans son autobiographie la série comme « des prises de bec et des bouleversements domestiques joyeusement résolus, une exagération de l'American life, [1'] amour personnifié ${ }^{6} \gg$. Les représentations y sont explosives pour deux raisons. Premièrement, Lucy n'aime pas ces tâches ménagères auxquelles elle préfère la sphère professionnelle du spectacle, et surtout, elle le dit haut et fort. Les narrations sont d'autant plus surprenantes que leurs conclusions tendent à récompenser la persévérance de l'héroïne qui parvient bien souvent à accéder à la scène du spectacle et à performer un numéro de chant, de danse ou de clown. La deuxième charge explosive de la série a trait à la dénaturalisation du travail domestique : non seulement l'héroüne n'aime pas s'en préoccuper mais elle y est de surcroît très mauvaise. Dans une scène mémorable, le spectateur est encouragé à rire du fait que Lucy, enceinte, tuerait certainement son enfant si elle le traitait avec autant d'étourderie et de brutalité que le baigneur sur lequel elle s'entraîne. Ces insuffisances ne se limitent pas à la pratique théorique sur une poupée. Épuisée par son nouveau-né, Lucy supplie très vite Ricky de se lever la nuit pour s'en occuper aussi : que ce soit le foyer ou la maternité, I Love Lucy montre les ennuis et les difficultés du métier, une monstration que les inaptitudes de Lucy rendent possibles.

Si son rêve est d'être une femme de spectacle, là non plus Lucy ne brille pas. Elle chante mal, danse le charleston quand il faut s'entraîner au classique, et n'est finalement crédible que lorsque son corps désarticulé et 
maladroit doit être celui d'un clown. Cependant si la force de Lucy ne réside pas dans ses talents, elle se loge assurément dans une détermination jusqu'ici réservée au masculin : n'acceptant jamais de s'entendre dire non, elle persévère, espérant un jour faire tomber normes et bornes patriarcales pour s'accomplir. L'absence de talents ménagers d'une part, et son obstination à réussir, valeur très étasunienne, d'autre part, obligent bien vite Ricky à révéler la pauvreté argumentaire de ses refus : s'il refuse à Lucy de sortir du foyer, ce n'est pas parce qu'elle est douée pour s'en occuper, mais bien parce qu'il veut « une épouse qui soit juste une épouse ${ }^{7} \gg$, réponse dont la tautologie montre bien l'arbitraire idéologique.

Héroïne d'inspiration beauvoirienne, qui n'est pas née femme, qui ne l'est pas totalement devenue et qui ne souhaite pas le devenir, Lucy est consciente d'occuper une place subalterne qu'elle tente de subvertir par l'humour. La série est faite d'actes et de sketches dits « de situation » prenant pour thèmes des confrontations hommes/femmes mêlés à des inspirations slapsticks héritées de la comedia dell'arte. Pour Patricia Mellencamp, l'humour de I Love Lucy est une réponse aux « conditions répressives des années 1950 [en tant qu'il est] l'arme et la technique de survie des femmes qui garantit la santé mentale, le triomphe de l'ego et le plaisir ${ }^{8} \gg$. Dans le même temps, les mouvements burlesques de Lucy encouragent des clowneries à l'idéologie syncrétique : Lucy est un bouffon et, à ce titre, ses capacités d'agir ont beau être débordantes, elles sont limitées par le cadre dans lequel elles s'expriment. Comme le souligne Arnaud Mercier, le bouffon a un statut ambivalent, « intercesseur entre les gouvernants et les gouvernés ${ }^{9} \gg$, qui oscille entre l'outrancier que le carnavalesque rend éphémère et aisément disqualifiable et une contestation dérisoire que le pouvoir plus lourdement armé peut écraser. Le corps incontrôlable de Lucy et sa persévérance montrent les impuissances de Ricky, dont le machisme est appuyé par l'étrangeté cubaine de ce personnage privé de langage intelligible ${ }^{10}$. Lucy a beau perdre narrativement les combats en revenant au foyer à la fin de chaque épisode, elle gagne performativement en restant sans cesse hors du contrôle patriarcal ${ }^{11}$.

La capacité de Lucy à passer outre les arguments « raisonnables » (en réalité réactionnaires) a mis au cœur de la première sitcom domestique et de la première héroïne télévisuelle des tentatives d'articulation particulièrement innovante. À défaut de pouvoir monter sur scène dans la sphère publique, Lucy Ricardo a, la première, amené la sphère publique dans la 
sphère privée en transformant son foyer familial en vaudeville. Une telle tentative d'articulation est absente de ses contemporaines comme Ma Sorcière Bien-Aimée ou le Donna Reed Show où les hérö̈nes ont explicitement choisi la sphère privée ; elle le sera aussi des femmes actives du Mary Tyler Moore Show ou de Murphy Brown pour qui la carrière prime. Même les hérö̈nes des années 1990 peinent, c'est justement tout leur propos, à cumuler réussite professionnelle et bonheur amoureux. Ces tentatives sont permises par la distinction que fait l'héroïne entre l'amour qu'elle porte à son époux et la structure conjugale dans laquelle ils sont engagés. Les frustrations du mariage, les vengeances quotidiennes, la guerre des sexes étaient des thèmes très récurrents de la série qui dévoilaient la capacité d'agir d'une femme au foyer révoltée. Lorsqu'elle ne répondait pas en une timide provocation « oui, Monsieur » à son époux, Lucy tournait ses ordres en dérision, s'affichant explicitement comme subalterne : s'insurgeant de ce que son épouse a invité des gens sans le prévenir, Ricky est moqué : «Oh je suis tellement désolée, j'ai oublié de Vous demander Votre permission, Votre Majesté ! ${ }^{12} \gg$ Quelques épisodes traitent même d'une redistribution du pouvoir, comme lorsque Lucy et son amie Ethel demandent à être «traitées exactement comme si elles étaient des hommes $\gg$ dans un épisode au titre explicite, « Equal Rights ${ }^{13} \gg$.

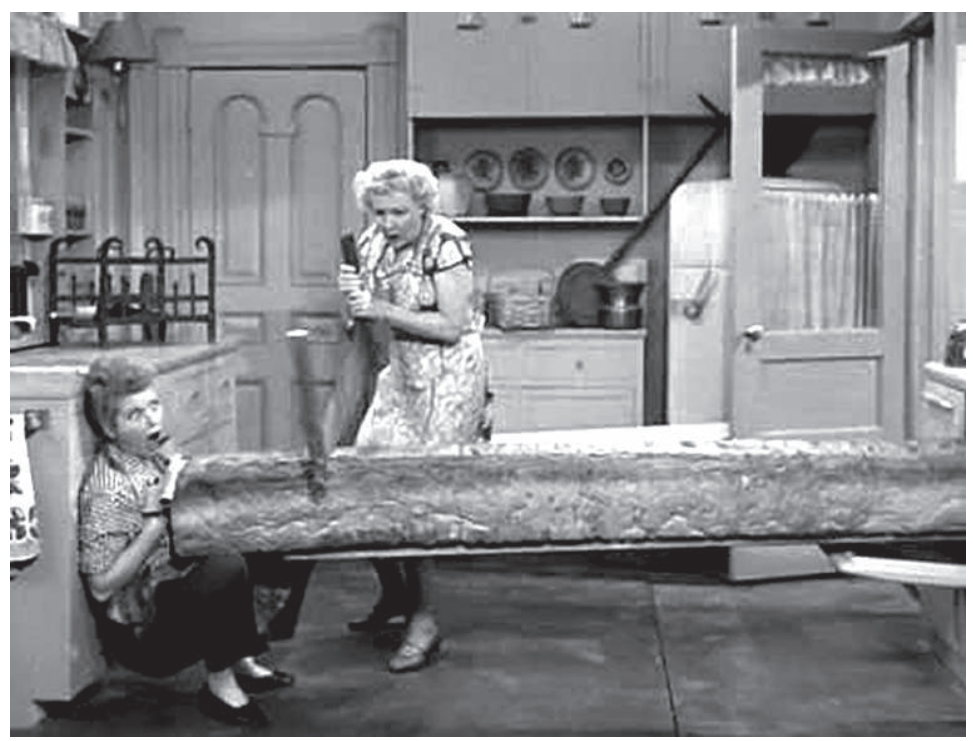


Quelques grivoiseries métaphoriques appuient la sujétion patriarcale que représente le travail domestique, comme lorsque Lucy se trouve poussée dos au mur par un pain phallique sortant du four, n'étant libérée que par la castration symbolique qu'opère son amie Ethel armée d'une scie ${ }^{14}$. La relation d'amitié qui lie Lucy à Ethel, unies dans l'adversité patriarcale, rappelle les principes de solidarité féminine des courants féministes mais a également, de façon plus surprenante, encouragé une lecture lesbienne de leurs interactions auprès de certains publics - y compris l'époux de l'interprète d'Ethel $^{15}$. Vivian Vance raconte dans son autobiographie que son mari voyait d'un mauvais œil la grande connivence qui existait entre elle et Lucille Ball : « "les gens commencent à parler de vous deux", me disait-il, "vous devriez faire attention à vos étreintes et vos embrassades" ${ }^{\text {"16 }}$ ».

Lucille Ball cesse en 1960 d'interpréter Lucy Ricardo mais elle n'abandonne pas l'alter ego Lucy, à qui elle donne d'autres séries et d'autres identités. L'héroïne grimpe chaque fois un barreau sur l'échelle de l'émancipation : de 1962 à 1968, Ball incarne dans The Lucy Show une veuve, Lucy Carmichael, vivant avec son amie (veuve, elle aussi) et ses deux enfants. En 1968, dans Here's Lucy, elle devient Lucy Carter, femme active dans une agence pour l'emploi, enfin dans Life with Lucy, elle est Lucy Barker, non plus employée, mais propriétaire d'un magasin d'outillage. Accompagnant les mutations socioéconomiques des femmes émancipées, Lucille Ball a lancé une charge féroce contre les antagonismes de la tradition puis de la première modernité et leur a fait subir des tentatives de réarticulation en montrant l'humour d'une femme, de surcroît d'une femme au foyer, faire de la sphère domestique une sphère théâtrale.

\section{L'amour domestique du Donna Reed Show}

Après les rébellions domestiques de Lucy Ricardo, mais aussi de Gracie Allen (The Burns and Allen Show), d'Alice Kramden (The Honeymooners) ou de Molly Goldberg (The Golbergs) à la sortie de la guerre, la fin des années 1950 marque le backlash d'une idéologie patriarcale aux sphères bien distinctes et aux rôles traditionnels. Aux bouffonneries et à la comptabilité catastrophique de Lucy, succèdent la grâce et le parfait ménage de Donna Stone (The Donna Reed Show), mais aussi de Margaret Anderson (Father Knows Best) et de June Cleaver (Leave it to Beaver) ${ }^{17}$. 
Le vaudeville de I Love Lucy se dissipe ainsi pour laisser place à un ton bien plus didactique, dénué de poursuites, de corps indomptables et de dérisions burlesques. Les quelques boutades envoyées par l'épouse au patriarche sont suivies d'un sourire bienveillant et, plutôt que de montrer l'ennui des tâches ménagères et les tentatives pour y échapper, la série revalorise le travail domestique et la figure de la femme au foyer : le scénariste William Roberts admet lui-même avoir voulu insister sur les nombreuses demandes faites aux femmes d'être des « épouses, mères, compagnes, entraîneures, nourrices, femmes de ménage, cuisinières, blanchisseuses, jardinières, comptables, femmes de club, chanteuses de chœur, parents d'élève, cheffes-scouts et d'être en même temps effervescentes, immaculées et jolies ${ }^{18} \gg$. Si le proto-féminisme de Lucy Ricardo faisait écho avant l'heure aux revendications de la deuxième vague, Donna Stone proposait plutôt un embryonnaire néoféminisme, une reconsidération méliorative du travail féminin, si bien qu'il fut d'ailleurs considéré pendant un temps de nommer la série en écho au patriarcal Father Knows Best (traduit en français par Papa a raison), «Mother Knows Better ${ }^{19} »$.

Amoureuse de son époux avec lequel elle échange de nombreuses marques d'affection, Donna Stone apparaît comme la clef de voûte du ménage familial, une fonction pour laquelle elle ne reçoit que peu de reconnaissance. L'amour n'est pas seulement sanctionné par le mariage, il s'y fond entièrement jusqu'à voir ses caractéristiques modelées par les impératifs de la vie conjugale et domestique telle que la pense la première modernité, qui écrase les tentatives subjectivantes des personnages. Dans un épisode très éloquent, Alex reconnaît sa réticence à voir sa femme monter un commerce, non pas, c'est ce que pensait Donna et c'est la raison pour laquelle elle apparaît si fermée durant tout l'épisode, parce qu'il pense qu'elle va échouer mais parce qu'elle est si douée aux travaux domestiques qu' il veut la conserver à son service. Quand elle l'apprend, Donna est aux anges et se prend au cou de son époux stoïque (une image qui ne manque pas d'accentuer les inégalités émotionnelles). Quand Alex « reconnaî $[\mathrm{t}]$ que c'est du pur égoïsme de $[\mathrm{s}] \mathrm{a}$ part », elle est ravie : «Oh, c'est l'égoïsme le moins égoïste que j'aie jamais vu ! »

Ces représentations idylliques étaient défendues par Donna Reed elle-même qui influençait très largement les

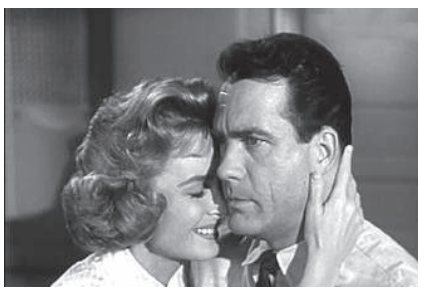


scripts et le casting de sa série, amenant de nombreux collègues à la qualifier de féministe ${ }^{20}$. Pourtant, si l'engagement de Donna Reed dans l'écriture et la production de la série rappelle celui de Lucille Ball, il témoigne des aspérités au sein même des subalternes puisqu'à l'opposé de Lucy Ricardo, Donna Stone se veut femme modèle et bienséante, ce que Reed formulait elle-même lorsqu'elle se disait « fatiguée de toutes ces sexualités immatures et de ces histoires de femmes loufoques, amorales et de mauvais goût ${ }^{21} \gg$. Personnalité puissante de la télévision, à cet égard précurseure des combats féministes de la deuxième modernité, Reed défendait conjointement une revalorisation des femmes et des politiques traditionnelles du patriarcat.

Reprenant à son compte le mythe de la pureté féminine, la série proposait une héroïne aux allures victoriennes, chaste et asexuée, comblée par la vie domestique et familiale. Bien incapable des métaphores sexuelles comme I Love Lucy et Ma Sorcière bien-aimée, les références sexuelles de la série se limitent à s'asseoir docilement sur les genoux de son époux ou à quelque mordillage d'oreille pour le réveiller mais Donna Stone n'en était pas pour autant dépourvue de capacité d'agir. Exprimant par endroits les frustrations de sa vie domestique, la série n'était pas ignorante de son mode de représentation idyllique de la vie domestique, les prenant parfois dans un mouvement autotélique comme sujet de son récit. Lorsque des amis du couple sont invités à dîner et ne tarissent pas d'éloges sur la tenue, la coiffure et la grâce de Donna, celle-ci finit par être exaspérée car elle perçoit

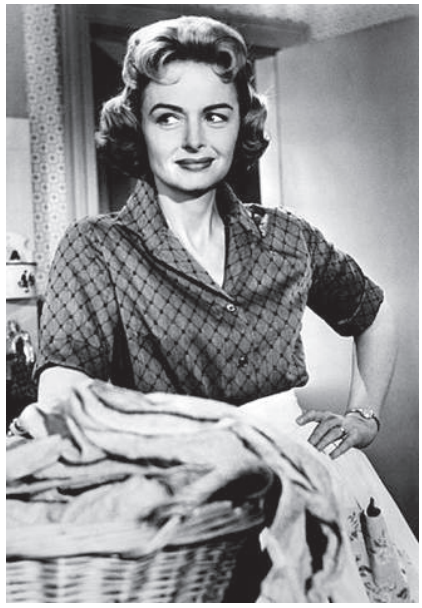
dans la qualification d'« adorable » une forme d'apitoiement - comme le miroir de sa sujétion. Elle décide de durcir le ton auprès de ses enfants et mari pour être immédiatement obéie, ajoute « non, un nouveau mot à $[s]$ on vocabulaire » et invoque son ras-le-bol d'avoir été « utilisée, victimisée et exploitée $e^{22} \gg$. Si l'ensemble de la famille se soumet vite à cette nouvelle autorité, ce n'est pas sans répercussions sur les relations entre les membres de la famille d'un côté et la mère de l'autre : Donna réalise qu'à crier et donner des ordres, elle a perdu les interactions douces et aimantes. Le coût pour que l'on cesse de « profiter » de sa gentillesse est celui-là même que les profiteurs payaient et cessent de payer, à savoir l'amour et la tendresse. Réalisant que ses interactions sociales, avec ses enfants comme avec son teinturier, 
s'appauvrissent en sourires et en proximité, Donna Reed réintègre immédiatement sa place et l'épisode se clôt sur cadeaux aux enfants et repas préféré de l'époux. Même si, en réponse au soulagement de son mari de voir « cette révolution finie et la population revenir à sa vie tranquille », Donna prévient que « ce n'est pas parce qu'elle s'est laissée attendrir que l'on reviendra tous à nos anciens comportements », la structure de la série faite d'épisodes isolés les uns des autres rassure sur les inconséquences de cette insurrection. La réaffirmation identitaire de Donna Stone, forme d'essentialisme stratégique lui permettant d'asseoir un pouvoir domestique au sein de la maisonnée, achoppe sur la figure patriarcale de son époux. Le pouvoir d'agir de la subalterne existe bien mais il trouve ses limites, non pas tant entre les murs de la maisonnée dont I Love Lucy puis Ma Sorcière bien-aimée montrent les possibles redéfinitions et porosités, mais auprès de l'époux et des modalités amoureuses qui les lient. Défini comme un care oblatif, l'amour agit comme un opérateur de reconnaissance et légitime le sacrifice personnel du féminin. Il est tautologique : Donna est dépendante de la dépendance d'autrui. Elle désire être désirée. Les routines immuables, l'absorption du « nous » dans le « je » masculin, la condition du « je » féminin à l'amour familial sont autant de signes d'une subjectivation féminine prise au piège de la revalorisation de ses compétences et dont l'identité du soi absorbée dans l'autre garantit la sécurité ontologique.

\section{Ma Sorcière bien-aimée, émancipations contradictoires et subversions magiques}

Si de prime abord, Ma Sorcière bien-aimée semble n'évoquer que le quotidien d'une sorcière déchue et contrainte par les idéaux réactionnaires de son mari, la série révèle rapidement une lutte constante entre le rêve américain romantique incarné par la relation entre Samantha et son époux, rêve fait de maisons suburbaines, d'enfants et de tâches domestiques, et l'ordre holiste, incarné par les liens entre Samantha et sa mère Endora, fait d'union familiale et de destins magiques.

Les émancipations sont sans cesse contrariées et contrariantes. D’un côté, les actions de Samantha sont réfrénées

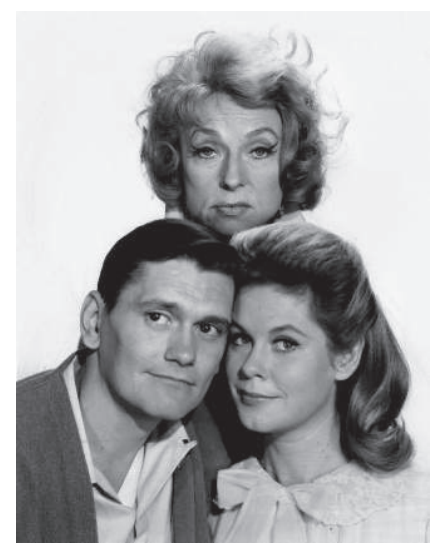


dans un mariage qu'elle a choisi tandis que ses pouvoirs débordent constamment les cadres autorisés par son mari, lequel subit la magie comme une revanche plus ou moins consciente des capacités d'agir bridées. De l'autre, Samantha est perçue comme un mouton égaré, une aliénée à libérer, par les membres de sa famille, que ce soit ses parents Endora et Maurice ou sa cousine Serena ${ }^{23}$. Ceux-ci se rappellent à elle en envahissant sans cesse et sans prévenir le foyer familial malgré les contestations d'un Jean-Pierre au pouvoir patriarcal finalement très affaibli. Souvent impuissant, celui-ci incarne aux yeux des sorciers un sous-être qui a déclassé Samantha : à de nombreuses reprises, la sorcière bien-aimée est montrée comme génétiquement et socialement supérieure à son époux.

Sa mère, Endora, critique ses choix de vie sur des arguments tour à tour progressistes et conservateurs, féministes et familialistes. Ulcérée de voir sa fille ainsi réduite au service de son époux, comme elle le signale en transformant ses habits en loques ou en l'enfermant dans une cage, Endora est aussi scandalisée de voir que Samantha s'émancipe de sa famille en renonçant à ses pouvoirs magiques et aux traditions qui les accompagnent. Endora incarne l'idéologie féministe en défendant l'émancipation des femmes : elle-même entretient une relation libre avec son mari, avec qui elle ne cohabite pas et contre lequel elle menace de « remplir un interlocutoire ectoplasmique $^{24} \gg-$ formule cryptique pour évoquer le divorce. Elle enjoint sans cesse à sa fille de renoncer à la première modernité pour retrouver une place de femme libre... dans les limites permises par sa famille. Quand Endora pense que Samantha quitte Jean-Pierre, sa jubilation est infantilisante : $\ll j$ 'ai toujours dit que ma petite fille reviendrait à moi ${ }^{25} \gg$. Dans le choix de l'amour patriarcal, Samantha à la fois s'émancipe et se soumet.

Le renoncement aux pouvoirs magiques, c'est-à-dire à la lignée familiale et à la supposée supériorité naturelle de Samantha, est légitimé par une articulation typique des imaginaires étasuniens de l'après-guerre, celle du rêve romantique avec le rêve américain. La conformité aux valeurs et idéaux du temps que sont l'achat d'une maison en banlieue, la division genrée des tâches, l'éducation des enfants et le couple aimant sont la promesse d'un bonheur familial qui récompense le travail de normalisation auquel se soumet Samantha. Quand Jean-Pierre demande à son épouse de se convertir aux comportements normaux, son argument est explicite : « La femme normale [doit utiliser] une transportation normale : ça fait partie du rêve 
américain $!^{26} \gg$ Pour l'époux traditionnel, le nez frétillant de Samantha est irrespectueux de la valeur travail qui sous-tend le rêve américain ; pire ! les pouvoirs magiques sont une menace contre la croyance en la capacité de chacun, moyennant travail et implication, à grimper les échelons sociaux. Concurrence déloyale, la magie déjoue les règles édictées par une société méritocratique. Quand Samantha publie grâce à ses pouvoirs une publicité pour aider un talentueux restaurateur, Jean-Pierre est furieux car non seulement la page de Samantha éclipse le travail publicitaire qu'il a fourni dans le même numéro mais, de surcroît, elle attente directement aux valeurs étasuniennes : en plus d'avoir heurté l'éditeur et les autres publicitaires, Jean-Pierre lui reproche de nuire « aux États-Unis d'Amérique, une démocratie dont le système économique s'appelle le capitalisme ${ }^{27}$ » ...rien de moins que cela.

Apprenant vite de ses erreurs, Samantha modifie son répertoire comportemental et clame peu à peu aimer le labeur domestique, préférer « le chemin mortel » qui apporte « un énorme sentiment d'accomplissement $^{28} \gg$. Tout au long de la série, on suit ainsi son apprentissage des tâches domestiques (de la cuisine à l'entretien ménager en passant par la peinture). Dans le couple romantique, Samantha n'est pas seulement assujettie au travail domestique que quelques écarts magiques viennent tout de même résoudre régulièrement, elle doit plus largement se conformer au système de valeurs de son époux.

Un tel processus n'est pas, c'est toute la face émancipatrice de la série, sans pierres d'achoppement. Au-delà des écarts de classe que le rêve américain allié à l'amour romantique entend dépasser, Ma Sorcière bien-aimée n'ignore pas les problématiques égalitaires. À de nombreuses reprises, l'hypothèse est formulée que l'interdiction de Jean-Pierre d'utiliser la magie serait une réaction égocentrique et jalouse, une tentative désespérée de contenir des capacités qui le dépasse : le père de Samantha souligne à l'égard de sa fille qu'« [il] ne veut pas [qu'elle] utilise [ses] pouvoirs naturels parce que ça le fait se sentir inférieur ${ }^{29} \gg$. Le pouvoir est clairement redistribué quand Samantha use, en cachette ou non, de facultés magiques qui agissent comme des leviers métaphoriques de l'émancipation féminine.

Lorsqu'elle est nommée Reine des sorcières au début de la quatrième saison, ses péripéties impliquent tout un ensemble de destins inéluctablement raccrochés au sien, au premier rang desquels ceux de sa mère et de son époux, qui ne sont pas seulement impliqués par effets de ricochets mais 
bien acteurs principaux du récit. Sa mère est convoquée, d'une part, puisque Samantha ne peut refuser car « c'est un grand honneur pour moi, et encore plus pour $\operatorname{maman}^{30} \gg$. Son époux, dont le pouvoir décisionnaire direct est balayé, entre tout de même dans le processus puisque, pour ne pas contrarier famille et enfants, Samantha accueille ses sujets pendant la nuit, créant pour compromis une classique double journée. Pour justifier sa nomination, la sorcière récupère les arguments du rêve américain que Jean-Pierre lui a inculqué ces quatre dernières années, plaçant également sa mère au cœur de sa décision : « Être reine est quelque chose dont chaque mère rêve pour sa petite fille, de la même manière que chaque père américain rêve qu'un jour, son garçon sera président ! » Si elle accepte, c'est donc explicitement pour réjouir sa mère et maintenir la lignée dont elle est issue, récupérant au passage à son compte les arguments patriotiques de Jean-Pierre. Au sortir d'une dispute, son mari se rend finalement à l'évidence, alors même que Samantha s'apprêtait à abdiquer pour le retrouver : « Être reine fait partie de ce que tu es. Et si je t'aime suffisamment, je dois l'accepter... (s'agenouillant, il poursuit :) Je suis à Votre disposition, Votre Majesté. »

Tandis que durant les premières années, Samantha doit s'adapter à la vie des mortels, dans les saisons plus avancées, le mouvement inverse se produit : Jean-Pierre reconnaît qu'il doit accepter la magie de Samantha, c'est-à-dire son affirmation individualiste, et ce « de la même façon que toi, tu acceptes certaines choses de mon monde ». Ce mouvement d'acceptation est accompagné d'un ensemble de signes d'émancipation : Samantha privilégie de plus en plus le pantalon aux symboliques jupons, elle engage une nounou-sorcière, et fait exponentiellement preuve d'argumentations dans des discussions qui se closent ainsi davantage sur des compromis que sur des arguments d'autorité patriarcale. 


\section{Notes}

1 Giddens, Anthony, La Transformation del'intimité. Sexualité, amour, et érotisme dans les sociétés modernes, Rodez, Éditions du Rouergue, 2004, p. 230.

2 I Love Lucy, saison 1, épisode 8.

3 I Love Lucy, saison 2, épisode 23.

4 Kaufmann, Jean-Claude, L'Étrange histoire de l'amour heureux, Paris, Fayard, 2010, p. 112.

5 I Love Lucy, saison 1, épisode 5.

6 Ball, Lucille, Love, Lucy, New York, New York, Putnam, 1996, p. 217.

7 I Love Lucy, saison 1, épisode 6.

8 Mellencamp, Patricia, «Situation Comedy, Feminism, and Freud: Discourses of Gracie and Lucy », in Butler, Jeremy, Star Texts: Image and Performance in Film and Television, Detroit, Wayne State University Press, 1991.

9 Mercier, Arnaud, «Quand le bouffon franchit le Rubicon : la candidature Coluche à la présidentielle de 1981 », Hermès, 29, 2001, p. 175-183.

10 Mellencamp, Patricia, ibid. On peut prendre l'épisode 4 de la saison 4 pour exemple des nombreuses moqueries de Lucy à l'égard du langage hésitant de son époux : «RICKY : Tu ne fais jamais ce que je te disais ! », « LUCY : Alors pourquoi n'arrêtes-tu pas de me le disais ? » (« RICKY: you never do what I told you! - LUCY: Then why don't you quit tolding $m e$ ? $\gg)$.

11 Mellencamp, Patricia, ibid.

12 I Love Lucy, saison 4, épisode 2.

13 I Love Lucy, saison 3, épisode 4.

14 Spangler, Lynn C., Television Women from Lucy to Friends. Fifty Years of Sitcom and Feminism, Santa Barbara, Greenwood Press, 2003, p. 36.

15 Doty, Alexander, « I Love Laverne and Shirley. Lesbian Narratives, Queer Pleasures, and Television Sitcoms », in Morreale, Joanne, Critiquing the Sitcom. A Reader, Syracuse, Syracuse University Press, 2002, p. 187.

16 Bragman, Bob, « I Love Ethel. A Vivian Vance archive uncovered », San Francisco Chronicle, 19 janvier 2010. 
17 The Donna Reed Show se distingue de ces deux dernières séries en ce qu'elle place l'épouse et mère au centre de ses récits.

18 Fultz, Jay, In Search of Donna Reed, Chicago, University of Iowa Press, 2001, p. 118.

19 Tucker, David C., The Women Who Made Television Funny: Ten Stars of 1950s Sitcoms, Jefferson, McFarland and Company, 2007, p. 118.

20 Leibman, Nina, Living Room Lectures: The Fifties Family in Film and Television, Austin, University of Texas Press, 1995, p. 182.

21 «Donna Reed, oscar winner and TV star, is dead at 64 », New York Times, 15 juillet 1986, référence faite à une interview du New York Times de 1964.

22 The Donna Reed Show, saison 1, épisode 25.

23 Serena est la cousine et sosie de Samantha. Alter ego émancipé, aux jupes courtes, cheveux bruns et souvent courts, elle incarne toutes les valeurs émancipatrices des années 1960 : Serena danse le rock, multiplie les aventures amoureuses et rejette toute forme de vie traditionnelle dont, explicitement, les enfants. Le contraste entre les deux personnages est accentué par le fait que, comme Samantha, Serena est interprétée par Elizabeth Montgomery.

24 Ma Sorcière bien-aimée, saison 5, épisode 28.

25 Ma Sorcière bien-aimée, saison 6, épisode 21.

26 Ma Sorcière bien-aimée, saison 1, épisode 26.

27 Ma Sorcière bien-aimée, saison 1, épisode 35.

28 Ma Sorcière bien-aimée, saison 2, épisode 24.

29 Ma Sorcière bien-aimée, saison 6, épisode 10.

30 Ma Sorcière bien-aimée, saison 4, épisode 1. 


\section{L'amour, \\ meilleur ennemi des femmes actives et des « féministes au foyer »}

\section{La montée des subjectivités féminines}

À la suite des femmes au foyer plus ou moins satisfaites de leur condition, les années 1970 et 1980 signent l'arrivée d'héroïnes, au sens fort du terme ${ }^{1}$ : les femmes sont au cœur des récits, comme des centres de gravité auxquels renvoient tous les autres personnages. Les subjectivités féminines elles aussi, dans leur hétérogénéité, passent des marges au centre de ces récits en qualité explicite de subalternes (il suffit de voir Roseanne, épuisée, crier sa double journée sur son époux pour s'en convaincre !). Les séries télévisées n'enrobent d'ailleurs plus leurs noms d'un «I Love » prononcé par le masculin mais portent franchement le prénom ou le nom de leur interprète, The Mary Tyler Moore Show, Murphy Brown, Maude, Roseanne, comme les étendards d'un processus subjectivant enclenché. L'hétérogénéité des courants féministes y est bien souvent ramenée à la grande bataille étasunienne entre les libérales et les radicales, incarnant les premières dans des working women qui conquièrent en priorité, mais non de manière exclusive, l'égalité de droit par la sphère publique, et les secondes dans des femmes au foyer et mères attachées à ne plus se laisser faire dans la sphère privée. Le genre se trouble peu à peu, à commencer par les puissantes femmes au foyer et mères Maude et Roseanne, herculéennes dans leurs gestions presque matriarcales du foyer.

Une telle individualisation des personnages participe du tournant féministe effectué par ces imaginaires. Alors que les premières femmes au foyer se battaient pour obtenir les mêmes égalités de droit que les hommes, les 
héroïnes des décennies 1970-1980 déplacent la condition de leur contentement vers l'épanouissement personnel. L'amour n'apparaît plus comme l'unique territoire où être reconnue (des fonctions que remplissent désormais le travail et l'amitié), et de structure il devient modalité interpersonnelle, avec des apports mais aussi des coûts. Deux ouvrages sont particulièrement importants dans l'analyse des héroïnes de cultures populaires des années 1960 : celui de Katherine Lehman², qui se concentre sur la figure de la femme seule à partir des années 1960, et celui de Bonnie $\mathrm{Dow}^{3}$, qui scrute les manifestations du féminisme auprès des héroïnes télévisées des années 1970.

Dans Those Girls. Single Women in Sixties and Seventies Popular Culture, Katherine Lehman analyse la montée de la « femme célibataire », « figure pivot dans la culture populaire de l'après-guerre [qui] a posé un affront direct au double standard sexuel et a défié la tendance dominante à l'égard du mariage anticipé des années $1960^{4}$ ». Selon Lehman, la femme seule représente les frustrations liées à la domesticité plus qu'une « réalité » sociale, des frustrations qui se manifestent dans les imaginaires sous la forme de stigmates, névroses et masculinisations. Une décennie plus tard, portées par la croissance démographique du célibat, les héroïnes se veulent plus féministes, bien que les industries médiatiques rechignent à investir dans un personnage principal féminin, peu enclines à croire en la capacité d'une femme à intéresser les publics, ce qui explique à la fois la relégation des héroïnes au genre comique, moins coûteux à produire, et le caractère mainstream de ces personnages de « femmes seules », blanches et de classe moyenne. La « femme célibataire » a pour topos le départ de sa ville natale, presque toujours provinciale, pour rejoindre l'anonymat et les possibilités démultipliées de la métropole. Cette fuite s'accompagne parfois d'une coupure brutale, mort de l'époux ou divorce (Diana, Fay, One Day at a Time, Phyllis, Alice), coupure que l'on retrouvera dans les années 2000 chez des quadragénaires veuves ou divorcées. Ce qui attire ces héroïnes, c'est certes la métropole mais aussi la fuite de l'environnement provincial et surtout familial qui enserre les comportements et les perspectives d'avenir. L'évasion des hérö̈nes, fut-elle à soixante kilomètres comme dans That Girl!, est donc le signe de leur individualisme naissant. Le féminisme de la deuxième vague ainsi représenté transforme par métonymie les villes en symboles des sphères professionnelles, symboles qui resteront à l'œuvre jusque dans les années 1980 et bien sûr 1990, lorsque Carrie Bradshaw 
(Sex and the City) emménage à New York et Ally McBeal à Boston, ou lorsque Lorelai Gilmore (Gilmore Girls) décampe à seize ans du froid foyer familial bourgeois.

À partir des années 1970, les femmes célibataires deviennent des working women. Cette conquête franche de la sphère professionnelle doit être comprise en tenant compte des mouvements féministes de la deuxième vague qui traversent alors la sphère publique : la très médiatique égérie Gloria Steinem, féministe libérale à la féminité et à la beauté rassurantes, n'est pas sans rappeler l'héroïne du Mary Tyler Moore Show. Selon Bonnie Dow, ces mouvements ont pu trouver une traduction dans les médias de masse du fait de leur inscription directe dans les valeurs américaines de l'individualisme et de l'égalité des chances ${ }^{5}$. Après la famille nucléaire, les séries télévisées proposent une «new woman », une « nouvelle femme » jeune, célibataire, hétérosexuelle, blanche, jolie, de classe moyenne et qui a réussi à investir la sphère professionnelle. La «nouvelle femme » ou la « femme seule » sont ainsi des traductions médiaculturelles des mouvements de libération féministes en même temps que leur profil très hégémonique témoigne des angles morts du féminisme de la deuxième vague, aveugle aux problématiques de diversité.

Il faut souligner que si le concept de «nouvelle femme » est particulièrement parlant pour évoquer cet ensemble de nouvelles propositions médiatiques, l'appellation n'est, en fait, pas nouvelle dans l'histoire des représentations socioculturelles des femmes. Pour Janet Lee, ce concept a servi de « technique de vente récurrente ${ }^{6} »$ pour promouvoir une image des femmes flexible mais durable : intrinsèquement liée à l'histoire des féminités, la « nouvelle femme » a d'abord désigné celle qui parvient à combiner la capacité à travailler et à prendre soin de la famille durant la deuxième guerre mondiale, puis l'épouse préférant rester à la maison au sortir de la guerre, enfin celle expérimentant la liberté sexuelle à partir des années 1960. Désignant tour à tour les femmes au foyer ou les femmes libérées, le terme s'avère historiquement réversible et désigne donc autant les incessantes constructions et hésitations des identités féminines que leurs liens avec les évolutions et les innovations socioéconomiques. Dans les fictions télévisées, les personnages sont appelés « nouvelle femme » lorsqu'ils abordent des thématiques jusqu' ici taboues comme l'avortement, le harcèlement sexuel, l'impotence ou la ménopause (Maude, Designing Women), innovation qui 
trouvera une formulation plus libérée encore, quelques décennies plus tard, dans Sex and the City. La « nouvelle femme » est également incarnée par des héroïnes dures, compétitives et franches comme Murphy Brown, qui se fichent de l'image qu'elles renvoient et cherchent une reconnaissance publique, non pas par l'appréciation de leur douceur et leur féminité, mais par leurs accomplissements professionnels. Enfin, elle est indépendante et individualiste dans One Day at a Time où les compagnons amoureux sont présentés comme une menace pour l'épanouissement ${ }^{7}$. Aussi, si la première working woman Mary Richards (The Mary Tyler Moore Show) apparaît à la télévision en 1970, ses influences intertextuelles remontent aux années 1960, dans la littérature et au cinéma notamment avec l'ouvrage Sex and the Single Girl ${ }^{8}$ qui, vendant deux millions de copies en trois semaines, encourageait les femmes à travailler, à avoir de nombreuses expériences sexuelles et à s'émanciper du mariage - en somme à transcender les plafonds de verre et les doubles standards sexuels ${ }^{9}$. Son auteure, Helen Gurley Brown, devient de 1965 à 1997 la rédactrice en chef de Cosmopolitan, participant explicitement de la consolidation de la figure de la « femme seule ». Elle promouvait la liberté sexuelle, la contraception, le divorce, l'activité professionnelle, conseillant les femmes sur les romances et la sexualité, les positionnant comme actrices de leur vie amoureuse. Au cinéma, l'adaptation de l'ouvrage avec Natalie Wood succède à l'émergence d'autres femmes en prise avec des interrogations féministes comme Doris Day (Pillow Talk, 1959) ou Kim Novak (Boys' Night Out, 1962) ${ }^{10}$, les cultures populaires s'appropriant les différentes étapes de la libération féminine, de la sexualité à l'abandon de la ville natale, jusqu'à la puissance d'agir des femmes actives et des femmes d'action des années 1970. Des séries comme Drôles de Dames (1976-1981), Police Woman (1974-1978), Get Christie Love (première héroïne solitaire noire, de 1974 à 1975), Wonder Woman (1976-1979) et Super Jaimie (1976-1978), qui mettent en scène une héroïne forte et active, marquent l'arrivée du drama dans une thématique jusqu'ici majoritairement traitée par des sitcoms dont la subversion reste alors cloîtrée aux limites de l'humour ${ }^{11}$. De telles représentations sont annonciatrices des combattantes des années 1990, comme la Tueuse de vampires Buffy ou l'espionne Sydney Bristow qui, pour désamorcer la méfiance de l'ennemi masculin, usent de leur blondeur ou de leur plastique - en somme, de leur apparente faiblesse. 
Les séries des années 1970 portent en elles des tiraillements entre le féminisme libéral, qui fait de la conquête de la sphère publique son combat privilégié, le féminisme radical, qui tente d'identifier et de renverser les fondements du système patriarcal, le néoféminisme essentialiste qui revalorisent les qualités féminines, et enfin, les prémisses postféministes qui interrogent l'héritage des combats de la fin des années 1960. Loin de l'atmosphère holiste des années 1950, l'individualisme se déploie donc petit à petit dans les séries télévisées à partir des années 1970. Comme pour prendre acte du tournant culturel, c'est-à-dire du décollement partiel des trajectoires socioéconomiques, politiques et culturelles, les liens entre les personnages, leurs carrières et leurs influences féministes ne répondent pas à une mécanique causale et encore moins à un déterminisme simple.

Dans Prime Time Feminism, Bonnie Dow fait de 1970 une année charnière qui élude les femmes au foyer des précédentes décennies pour se concentrer sur des femmes autonomes. Son étude porte sur cinq programmes qu'elle envisage comme des « phases de réactions aux féminismes $^{12} \gg$ : The Mary Tyler Moore Show et One Day at a Time pour le féminisme radical des années 1970, Murphy Brown et Designing Women pour la construction d'un backlash antiféministe dans la deuxième moitié des années 1980, et Dr Quinn, Femme médecin pour le féminisme matérialiste de la fin des années 1980 et du début des années 1990. Cette enquête constitue l'un des premiers ouvrages sur les traductions médiatiques des mouvements féministes. Les variations et l'hétérogénéité de ces derniers sont bien illustrées par l'ampleur historique du terrain et par l'inscription, de façon non mécanique, des représentations des hérö̈nes dans leur contexte socioculturel, chaque programme étant relié aux conjonctures féministes, à la fois juridiques et politiques. L'auteure voit dans ces séries un étouffement des politiques féministes de la deuxième vague, qu'elle définit comme militantes, publiques et collectives, au profit de personnages qui s'émancipent sans remettre en cause les structures patriarcales, estimant que l'indépendance est acquise à travers les choix individuels. Elspeth Probyn, en 1990, distingue aussi les combats collectifs des politiques de valorisation du choix personnel, qu'elle diagnostique comme un piège « reproduisant la solide nature du statu quo », «choiceoisie » illusoire qui n'offre en fait aucun choix puisque les structures patriarcales restent intactes ${ }^{13}$. Une telle liberté serait donc toute relative car la percée 
des personnages féminins dans la sphère publique est définie selon les termes préservés du système de genre traditionnel : une héroïne comme Mary Richards (The Mary Tyler Moore Show) a beau être productrice d'une émission de télévision, son travail est essentiellement celui d'une secrétaire qui prend soin de l'équipe avec tout le care caractéristique des femmes. En conséquence, le féminisme n'y serait pas, selon Dow, un cadre discursif mais une tonalité qui influence les récits. Il est vrai que les discours féministes tels qu'ils sont exprimés par les politiques militantes, c'est-à-dire en apparence cohérents et portés vers l'égalité, ne sont pas recalqués tels quels dans les fictions. Peu d'hérö̈nes saisissent explicitement les mouvements féministes, ce qui n'empêche aucunement qu'elles soient aux prises avec des questions féministes. Pourtant, dans la lignée d'une réflexion, comme a pu la structurer Éric Macé ${ }^{14}$, sur les médias de masse comme sphère publique fraserienne et non comme arène politico-militante ou espace public habermassien, il faut envisager les courants et contre-courants féministes qui s'expriment au fil de l'histoire des héroïnes comme des luttes de définition du monde, des affrontements gramsciens au sens où les hégémonies et les contre-hégémonies ne sont elles-mêmes pas unies.

Ces personnages de femmes actives portent en effet à la fois l'optimisme des promesses de la sphère publique et le pessimisme de l'obligation à formuler un choix entre public et privé. Concentrant ses efforts sur les égalités de droit, le triomphe du féminisme libéral dans les années 1970 aux États-Unis ouvre la voie à la figure de la working woman qui, pour autant qu'elle s'épanouisse dans son métier et n'ait plus d'arcs narratifs exclusivement amoureux ou familiaux, semble comme enfermée dans la sphère publique. Punition de l'émancipation ou ténacité de l'hermétisme public/privé ? Les working women ne sont pourtant pas frileuses aux sacrifices, elles qui passent des soirées entières à travailler, mais à l'heure de la montée des subjectivités féminines, si investissement oblatif il doit y avoir, il sera à visée individualiste. Ces rigidités dans l'articulation public/privé ont amené Bonnie Dow à qualifier Mary Richards et Murphy Brown de « postféministes », en ce qu'elles font de leur carrière professionnelle la clé de leur épanouissement, jouant ainsi dans les cadres du système patriarcal et re-légitimant les séparations entre privé et public. Mary et Murphy, à bien y regarder, réaffirmeraient les codes patriarcaux. Pourtant, l'hypothèse même selon laquelle les héroïnes ne brouillent aucunement les frontières 
public/privé peut largement être critiquée. Le syllogisme dichotomique qui associe le privé au féminin, le public au masculin et oppose ainsi le féminin au masculin est très entamé par la conquête féminine de la sphère publique à l'instar de Mary Richards ou, deux décennies plus tard, par la féminité masculine de Murphy Brown. Certaines barrières résistent bien sûr que Bonnie Dow met à jour, mais ces carrières efflorescentes révèlent qu'à rebours d'une hiérarchisation simple du public sur le privé bannissant ce dernier, les héroïnes des années 1970 puis 1980 construisent leurs identités dans une articulation entre public et privé qui, pour autant qu'elle soit oppositionnelle, parfois même compétitive et donc hiérarchisante, ne les divise pas mais les définit l'une par rapport à l'autre. Les amitiés formées avec les collègues, les attaches domestiques entre Murphy et son homme à tout faire, les soirées entre filles de Mary Richards... : tous ces liens agissent comme des micro-articulations positives dans l'articulation oppositionnelle entre privé et public. Il apparaît essentiel de revenir quelques pages durant sur les représentations proposées dans The Mary Tyler Moore Show et dans Murphy Brown pour comprendre les politiques professionnelles et amoureuses qui y sont à l'œuvre, et ce, sans anachronisme.

\section{Dans The Mary Tyler Moore Show, être célibataire n'est pas être seule}

Actrice de son existence, femme active, célibataire sans en pleurer, Mary Richards incarne en 1970 un fort renouveau dans les représentations des héroïnes. Détachée des structures familiales, qu'elles soient celles d'origine ou celles à construire, Mary fait souffler le vent de la deuxième modernité par la force de ses liens amicaux et professionnels ainsi que par la prédominance des problématiques du bonheur et de l'épanouissement de l'individu. Le générique de The Mary Tyler Moore Show illustre ce paradigme individualiste et féministe dans lequel vient s' insérer l'héroïne après la rupture amoureuse liminaire de la série. Mary Richards, trente ans, conduit tout sourire une voiture vers Minneapolis. Les images se succèdent de son appartement et de ses amies, de son travail et de promenades dans le parc, pour terminer sur un plan de l' héroïne tournoyant sur elle-même puis jetant en l'air son bonnet ${ }^{15}$, geste bien connu des Étasuniens diplômés qui signifient ainsi le passage vers une autre vie. La chanson qui accompagne 


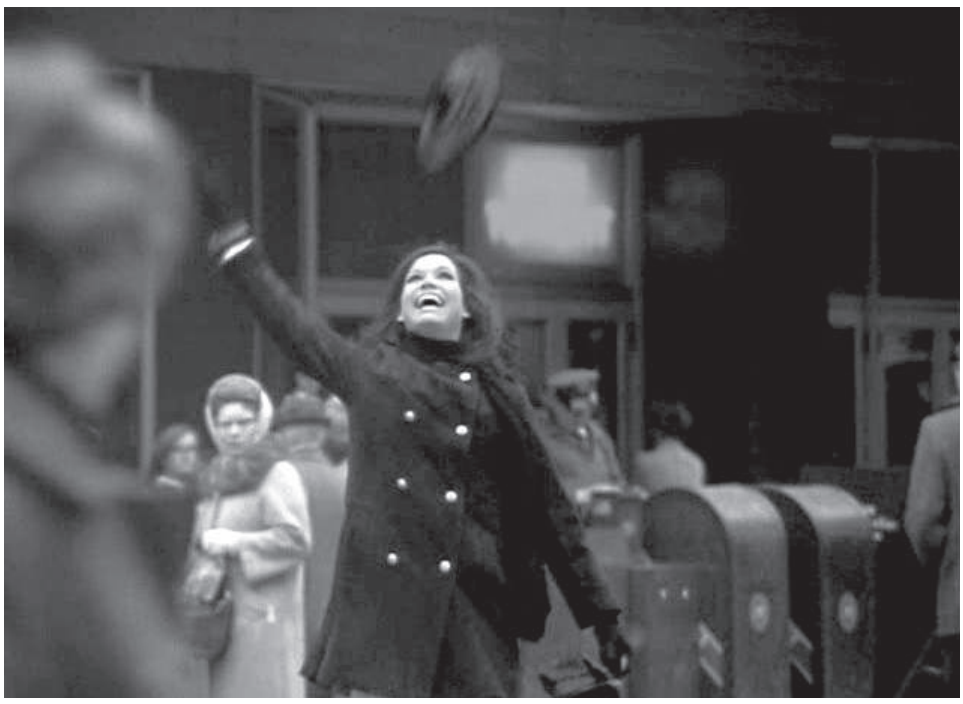

ces images d'une émancipation évoque les angoisses du succès individualiste en parallèle de la joie de vivre affichée par l'héroïne : «Comment vas-tu y arriver ? Le monde est affreusement grand et, ma fille, cette fois tu es toute seule. Mais il est temps de commencer à vivre, il est temps de laisser quelqu'un d'autre donner. L'amour est partout autour, pas besoin de le simuler, tu peux avoir la ville, pourquoi ne la prends-tu pas? »Comme un mantra, Mary jette son chapeau en l'air tandis que la chanson répète : $\ll$ tu vas y $\operatorname{arriver}^{16} \gg$. Cette image d'une femme virevoltant sur elle-même dans la rue sera reprise par Carrie Bradshaw dans le générique de Sex and the City et annonce déjà l'urbanisme très marqué des années 1990, tant dans Sex and the City que dans Ally McBeal. L'épiphanie individualiste de Mary surgit d'une déception amoureuse. Dans le premier épisode, l'hérö̈ne déménage vers Minneapolis pour commencer une nouvelle vie après avoir rompu avec son petit-ami qu'elle a financièrement soutenu durant ses études de médecine et à l'issue desquelles l'héroïne espérait le mariage. Le téléspectateur aperçoit durant le pilote l'épilogue de cette relation lorsque son ex rend visite à Mary dans son nouvel appartement, lui offrant des fleurs volées du chevet de l'un de ses malades. L'héroïne, prête pour un nouvel envol, réalise que ce mariage, avec cet homme, n'est pas dans ses intérêts et rompt avec lui. Avec une détermination toute nouvelle à la télévision 


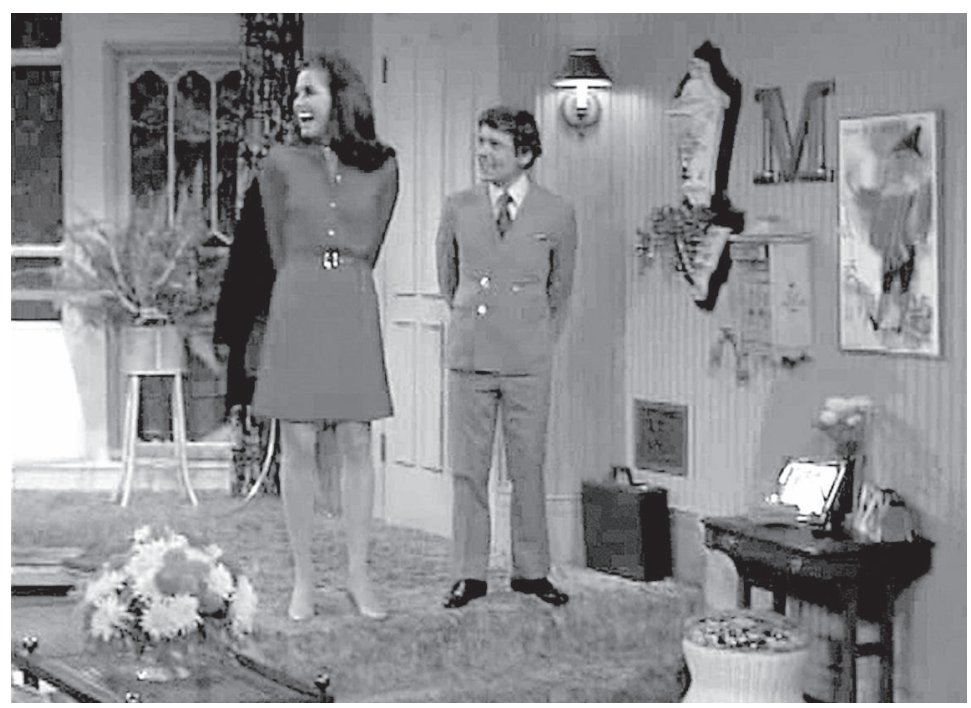

étasunienne, lorsque son ex-petit-ami lui dit de « prendre soin d'[elle] », Mary répond, lapidaire mais calme : «Je crois que c'est ce que je viens de faire $^{17}$. » The Mary Tyler Moore Show surprend ainsi par la remarquable absence de petits-amis : de nombreux épisodes s'écoulent sans que l'héroïne ait une quelconque intrigue amoureuse et lorsqu'elle en a une, il ne s'agit pas d'une relation stable mais de dates, de rendez-vous amoureux qui l'engagent peu. Certains d'entre eux représentent les catastrophes relationnelles qui feront les choux gras des célibataires très sexualisées de Sex and the City, d'autres se terminent harmonieusement, mais dans la majorité des cas, les hommes en question sont évincés sans justification, ne réapparaissant même pas dans l'épisode suivant. Le fait que Mary, presqu' invariablement, les invite à monter chez elle à la fin du rendez-vous provient certes de la facilité logistique à ne pas multiplier les décors et surtout à ne pas avoir de décors extérieurs (l'héroïne partage son temps entre son appartement et son travail), mais laisse aussi planer l'ambiguïté sur ses activités nocturnes à l'heure où la sexualité pré-maritale est encore taboue sur les écrans étasuniens. Dès les tout premiers épisodes et donnant ainsi le ton de la série avant même qu'elle ne soit confortablement installée dans les grilles de programmation, les hommes que Mary Richards rencontre traduisent une forme très embryonnaire des masculinités déstabilisées qui, là encore, 
peupleront les univers de Sex and the City ou Ally McBeal : l'un est excessif dans son expressivité et son attention (« C'est trop ! Trop d'amour, trop de compréhension, trop de générosité, trop ! », supplie Mary) ${ }^{18}$, un autre est plus petit qu'elle ${ }^{19}$, un dernier se bataille de façon infantile avec ses parents qui ne reconnaissent pas l'importance de son métier pourtant prestigieux ${ }^{20}$. Ces nouvelles identités masculines, combinées à l'implicite liberté sexuelle de Mary, font de cette dernière bien plus qu'une ancêtre de Carrie Bradshaw et d'Ally McBeal : elle en est, en réalité, une puissante matrice représentationnelle.

Déjà dans cette série, à l'orée des années 1970, la réflexivité sur les identités passe par le constat d'une réduction des femmes à leur statut de célibataire : «Je pourrais découvrir le secret de l'immortalité et les gens diraient toujours "Regardez cette fille célibataire qui a découvert le secret de l'immortalité !” », se plaint Mary ${ }^{21}$. L'amalgame du célibat et de la solitude est brisé par l'héroïne et son entourage, les liens faibles révélant leur force tant avec ses collègues que, dans le dernier épisode, elle appelle sa famille, qu'avec ses amies avec lesquelles elle passe ses soirées. Mary est célibataire mais elle n'est pas seule, elle refuse aussi la stigmatisation qui accompagne ce statut pour en revaloriser le potentiel épanouissant. À la fin de la série, Mary Richards a trente-sept ans et n'a toujours pas, contrairement à Ann Mary promise à l'autel (That Girl!), de relation durable. Cette innovation est certainement due à l'exceptionnelle présence de scénaristes femmes dans l'équipe de production. Contrairement à ses contemporaines, The Mary Tyler Moore Show réussissait en 1973 le défi de la parité : la moitié de ses scénarios avaient été pensés et écrits par des femmes, certaines d'entre elles remportant même pour la première fois un prestigieux Emmy Award ${ }^{22}$. Pour autant, les créateurs de la série, Allan Burns et James L. Brooks, niaient toute influence militante : même s'ils cohabitaient, « nous n'épousions pas les mouvements féministes [mais] nous voulions montrer quelqu'un avec les origines de Mary Richards évoluer dans un monde où les droits des femmes étaient en discussion et avaient un impact ${ }^{23} \gg$. Le recrutement de femmes scénaristes a inspiré aux scénarios, sinon une fibre féministe, du moins les interrogations, les espoirs et les réflexions d'une expérience féminine. Aussi Bonnie Dow a-t-elle raison de souligner que Mary, à bien des égards, réaffirme des codes patriarcaux - notamment par sa fonction de mère au travail ou par la réaffirmation de la dichotomie privé/public. Toutefois, 
son approche des mouvements hégémoniques et contre-hégémoniques héritée de Todd Gitlin selon laquelle les contenus médiatiques « absorbent [les mutations sociales] dans des formes compatibles avec le cœur de la structure idéologique ${ }^{24} \gg$ dominante se heurte aux mutations industrielles au cœur même de la structure idéologique non unifiée, sans parler des formes complexes de réception, négociées ou oppositionnelles, dégageant de nombreux niveaux de lectures.

\section{Murphy Brown, affirmation féminine de soi et dominations amoureuses}

Murphy Brown, de la série éponyme, est la deuxième grande figure du féminisme libéral de la télévision étasunienne. Lorsque la série se termine en 1998, Ally McBeal occupe déjà la grille de programmation de la FOX depuis un an et les héroïnes de Sex and the City s'apprêtent à débarquer. Tout en profitant du chemin déminé par ses prédécesseures comme Mary Richards, Murphy Brown annonce à sa façon les trentenaires célibataires de la deuxième moitié des années 1990 .

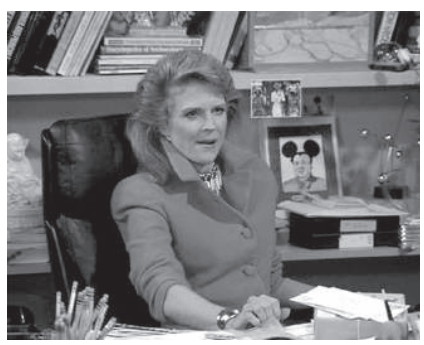
Célibataire explicitement féministe, le personnage est l'un des premiers à combiner une apparence féminine que marquent ses longs cheveux blonds avec un comportement masculin. Ambitieuse, parfois austère, toujours franche, Murphy ne porte pas un care démesuré à ses proches, tout en étant loyale, honnête et proche lorsqu'il le faut. Diane English, la créatrice de la série, explicite ainsi ses intentions : « Murphy était cette personne que l'on voulait toutes être. Toutes ces choses que vous regrettez de ne pas avoir dites ou que vous n'avez pas pu dire, elle les a dites. [...] Nous passons tellement de temps à essayer d'être appréciées, et à vouloir être appréciées... À cette époque, il était inhabituel de voir quelqu'un qui s'en fichait complètement. »

Alors que la porosité des genres n'avait pas atteint la très féminine et adorable Mary Richards et qu'elle s'expliquait, pour Maude puis Roseanne, par les corvées quotidiennes, la féminité masculine de Murphy Brown fait date. John Fiske a très bien souligné l'analogie implicite que faisaient à ce moment les Républicains entre Murphy Brown et Hillary Clinton, toutes deux « blondes, grandes, fines et actives, dans la fleur de l'âge, aux 
expressions faciales confiantes et au langage corporel assuré », toutes deux étant également de « nouvelles femmes qui détruiraient les [valeurs] traditionnelles ${ }^{25} \gg$. La montée des problématiques féministes trouvait à la fois écho et soutien dans la montée des subjectivités féminines fictionnelles, que vint soutenir une réappropriation de la masculinité. L'hérö̈ne est également innovante à travers les problèmes qu'elle rencontre dans le prologue de la série. Lors du pilote, Murphy revient d'un centre de désintoxication où elle a soigné son alcoolisme et renoncé à la cigarette : elle anticipe à cet égard les névroses des trentenaires ou les béquilles psychologiques (cigarette, alcool, pilules en tout genre) saisies par les quadragénaires à compter des années 2000. Ces caractéristiques étaient traditionnellement masculines, puisque l'alcoolisme avait déjà été abordé dans différentes séries, par exemple dans Maude, mais il n'atteignait que les hommes. Candice Bergen, l'actrice principale, témoigne de la difficulté qu'a eue la chaîne à accepter de telles caractéristiques : « à CBS, ils ont dit “plutôt que d'être une femme de quarante ans tout juste sortie de Betty Ford [célèbre centre de désintoxication], ne pourrait-elle pas être une femme de trente ans qui revient d'un spa ?" Diane [English, la créatrice de la série] a dit, "Non. Ce n'est pas ce que l'on veut dire." » Par sa voix grave, par sa kinésie masculine, par son assurance et son ambition, mais aussi par la montée de la «fatigue d'être soi » que cristallise son alcoolisme et sa dépendance à la cigarette, Murphy Brown osait dénaturaliser sa féminité et ce, sans devenir un monstre puisqu'elle s'affirmait sans écraser l'autre, qu'elle était ambitieuse sans arrivisme, et sérieuse sans agressivité. Murphy signait l'avènement d'une subjectivité féministe sans menace castratrice.

C'est finalement par Murphy que la problématique amoureuse est heurtée le plus violemment. Mary Richards restait un personnage finalement très doux et Maude et Roseanne, pour autant qu'elles contestent fortement la structure familiale et patriarcale, y restent largement enfermées. Les thématiques amoureuses sont en revanche traitées dans Murphy Brown au prisme très précis du pouvoir. Les échecs amoureux de Murphy sont dus à des luttes pour la domination : pour la première fois, les politiques amoureuses, et non familiales et domestiques comme dans Maude et Roseanne, sont abordées pour elles-mêmes. Il faut souligner un arc narratif important de Murphy Brown. Comme pour compenser la labilité moderne des relations amoureuses hétérosexuelles par un amour éternel 
et absolu, Murphy tombe enceinte à la fin de la troisième saison. Cette représentation proposant pour la première fois une femme qui choisit explicitement d'être mère célibataire a soulevé de vives protestations dans les camps politiques conservateurs. En 1992, le lendemain de la diffusion de l'épisode où Murphy, toujours célibataire, accouche, le vice-président étasunien et Républicain Dan Quayle prononce un discours vantant le mariage, dans lequel il critique violemment les choix maternels de Murphy Brown, regrettant qu'elle «moque l'importance du père, mette seule un enfant au monde et appelle juste ça un autre "choix de vie"26 ». L'affaire fera la une des journaux ${ }^{27}$, le Daily News allant jusqu’à titrer « Quayle à Murphy Brown : traînée ! » Bill Clinton, en campagne présidentielle à l'époque, entre

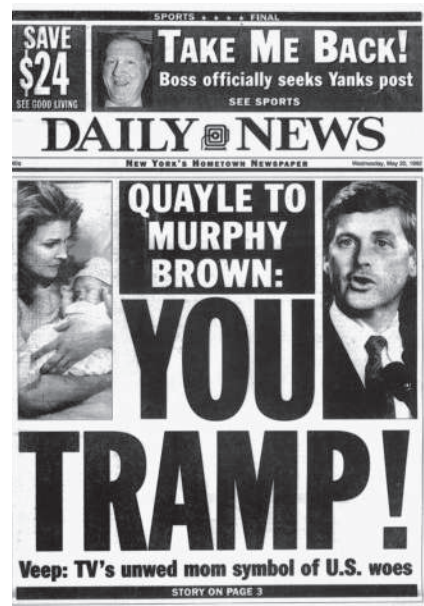
dans la polémique pour avancer que « des millions d'Américains pensent que [Murphy] a des choses pertinentes à dire ${ }^{28} \gg$. La grossesse se fait sans père mais pas sans réflexivité genrée et amoureuse. Cet arc narratif est la thématique au prisme duquel les relations amoureuses avec ses deux partenaires les plus sérieux, Jake et Jerry, sont évaluées puis évacuées ou au contraire préservées. Murphy est enceinte de Jake, qui ne souhaite pas assumer cet enfant et en laisse la pleine responsabilité à l' héroïne : en réalité, celle-ci ne « moque »donc pas l'importance du père, comme le soutient Quayle, puisque c'est bien ce dernier qui choisit de ne pas s'impliquer dans la parentalité. Le modèle amoureux plus instable, embryonnaire de la relation pure, se porte au secours de Murphy : Jerry propose à l'héroïne de l'accompagner dans sa grossesse en emménageant chez elle. Peu à peu et sans fiançailles, les deux personnages reprennent leur relation amoureuse. Les limites du romantisme sont ainsi explicitement compensées par les valeurs d'engagement et d'honnêteté ; finalement, l'amour conditionnel se montre plus fort et plus fiable que l'amour de principe. Toutefois, ce modèle aussi montre rapidement ses limites et se heurte à la vie quotidienne. Comme souvent dans les représentations de l'amour, la scène d'une dispute entre les personnages est révélatrice de toutes les tensions sous-jacentes à leur modèle et à leur structure de couple. Furieuse que Jerry ne soit pas rentrée à temps pour dîner alors qu'elle ne lui avait pas explicitement demandé, ce dernier enjoint à Murphy d'expliciter ses envies et ses besoins, d'être « honnête 
car l'honnêteté, ça fait tout », sans réaliser qu'il ouvre alors la boîte de Pandore $^{29}$. C'est un déferlement de sentiments qui se produit : Murphy assène tout ce qu'elle déteste chez lui, reconnaît ne pas savoir demander de l'aide car cela la fait se sentir « vulnérable » et dit détester « quémander » sans cesse. Jerry, en retour, admet que les contraintes quotidiennes du couple le font se sentir « claustrophobe », ce qui le fait en retour se sentir « coupable » et le rend « rancunier ». Réalisant qu' il n'aime pas vivre avec elle et qu'elle n'aime pas vivre avec lui, ils concluent « on peut pas faire plus honnête que ça, hein ? », constatant l'échec de leur idéal communicationnel. La démocratie relationnelle montre donc ses limites lorsque les discours, aussi honnêtes soient-ils, ne sont pas accompagnés de pratiques, et notamment de compromis. La séquence révèle une lutte profonde entre les intérêts contradictoires des deux identités de Murphy : la première est en manque d'affection, veut un compagnon avec qui dîner, se venge en ne faisant pas attention à son physique; la seconde est indépendante, sûre d'elle, et si autosuffisante qu'elle refuse d'accueillir quelqu'un.

Secrétaires ou grands reporters, ce n'est pas un hasard si ces femmes sont issues des classes moyennes supérieures (The Mary Tyler Moore Show), voire des classes supérieures (Murphy Brown) : l'épanouissement dans la sphère publique est un luxe que peuvent se permettre des classes dont le métier n'est pas seulement alimentaire. À contrario en effet, la sphère publique apparaît bien peu libératrice à Roseanne lorsqu'elle trie des morceaux de plastique sur les chaînes de son usine. Les intérêts entre la sphère publique et la sphère privée se révèlent contradictoires pour les femmes des classes populaires, comme le souligne le producteur de Roseanne, Tom Werner : «C'est un personnage qui est payé au taux horaire, qui n’est pas suffisamment couvert par la sécurité sociale, qui lutte et essaie de trouver un équilibre entre les besoins d'être une bonne mère et les besoins de ramener un salaire à la maison ${ }^{30}$. » L'épanouissement par le travail apparaît comme la manifestation à la fois d'une problématique de classe dans des récits où ces questions sont sous-représentées et d'une hégémonie des perspectives des classes supérieures sur les propositions féministes. À la manière des représentations françaises où les héroïnes avocates, policières ou médecins obtiennent l'égalité dans la légitimité de la sphère publique, les émancipations de Mary Richards et de Murphy Brown, dans la concentration de leurs efforts sur les égalités de droit, rendent invisibles certaines problématiques 
populaires que se chargent de traiter les héroïnes aux influences radicales comme Maude et Roseanne : plutôt que de gommer les distinctions, ces dernières insistent sur les différences d'expérience entre hommes et femmes, mettant l'accent sur les préjugés sexistes rencontrés au quotidien lorsque leurs époux ou patrons les disqualifient.

Ainsi, l'aliénation quotidienne éprouvée par Roseanne ou les difficultés que rencontre Maude pour obtenir un travail du fait de son manque de qualification et d'expérience tranchent avec les problématiques d'équité et de redistribution économique expérimentées par Mary et Murphy, femmes diplômées et éduquées, capables de concurrencer à compétences égales et sans autres formes de rééquilibrage la force de travail masculine. L'unique injustice contractuelle que rencontre Mary lors de son embauche est économique : «C'est un travail de productrice associée, lui dit son futur patron, ça paye dix dollars de moins par semaine qu'un travail de secrétaire et si vous pouvez descendre encore de quinze dollars, on vous fait productrice ${ }^{31}$. » La « vente » d'un statut élevé en échange d'une rémunération appauvrie est concédée par Mary (qui signale tout de même «n'avoir pas les moyens d'être productrice »!), désireuse après sa grande déception amoureuse de trouver une nouvelle vie dans les promesses de l'émancipation par la sphère publique, et suffisamment éduquée pour rencontrer l'opportunité d'un tel investissement. L'insistance sur les égalités de droit réactive et féminise la corrélation entre richesse financière et pauvreté interactionnelle, elle gomme aussi les thématiques de démocratie amoureuse, de réarticulations des sphères publiques et privées, de répartition égalitaire des tâches et des sacrifices. Il est d'ailleurs curieux que Bonnie Dow ne fasse qu'une référence fugace à Roseanne, argumentant en faveur du féminisme explicite de la série... alors qu'elle critique précisément, et à plusieurs reprises, la surreprésentation des femmes de classe moyenne. Cet écueil n'est pas la spécificité de Dow : à l'exception de quelques articles, les travaux historiques des représentations des héroïnes n’ont que peu traité cette série pourtant explosive à l'égard des figures de parfaites mères au foyer ou de femmes actives issues des classes supérieures, et à laquelle il faut donc accorder ici une place toute particulière. 


\section{La fin de l'abnégation féminine et le début des menaces castratrices}

La sphère privée est, avec Maude (1972) et Roseanne (1988), au cœur des récits comme le théâtre des articulations genre/amour. Parce qu'elles sont mariées et qu'elles vivent le quotidien domestique tout en travaillant en parallèle, ces héroïnes sont plus explosives vis-à-vis des modèles traditionnels amoureux que les réfugiées politiques Mary et Murphy qui s'isolent dans le travail. Quadragénaire de classe moyenne supérieure ou trentenaire ouvrière, les jupons et serre-têtes ne sont plus qu'un lointain souvenir : désormais, les femmes au foyer sont braillardes. Maude et Roseanne ont de la gouaille, disent ce qu'elles pensent et ne s'en excusent pas, écrasent bien souvent les membres de la famille sous un matriarcat légitimé par le travail qu'elles fournissent pour prendre soin d'eux.

La première désarticulation de ces nouvelles héroïnes a trait à la maternité et à la féminité. Le care, toujours très présent, n’est plus une bénédiction mais un labeur voire un fardeau lorsque les femmes sont seules à l'exécuter. Si elles aiment certes leurs enfants, elles montrent sans détours que la domesticité et l'éducation ne sont pas idylliques, critiquant l'ingratitude de leur progéniture par la moquerie et le sarcasme : «vite, ils sont partis, change les serrures! $\gg$, lance Roseanne à son époux dès que ses enfants sont en route pour l'école ${ }^{32}$. Conjointement à ces renouveaux maternels, les hérö̈nes troublent le genre tant dans leurs corps que dans leurs conduites : les gestes ne sont plus aériens mais brutaux, les pas sont énergiques sinon lourds, le ton monte souvent. N'envisageant plus le foyer familial par le prisme du rêve américain de la propriété privée mais comme le territoire du couple, les objets, lors des disputes, sont attrapés comme des symboles de la mise en commun et brisés, quand ils ne sont pas franchement envoyés à la figure du conjoint. Tout cela amène à ce que les revendications féministes apparaissent de façon très explicite, à la fois au cœur des récits et dans les discours des auteur.e.s. Roseanne Barr admet avoir été influencée par les représentations télévisuelles idéalistes des années 1950 et 1960, dont elle voulait repousser les limites. Barr, elle-même issu d'un milieu populaire juif, souhaitait montrer le quotidien « réaliste » des femmes ouvrières et ainsi revaloriser l'énorme travail produit par les mères : « Tout le monde sait que ce sont les femmes qui tiennent la famille mais ce n'était jamais 
montré. [Pourtant,] l'histoire d'une vraie mère à la télévision... je me suis dit "bon sang, c'est tellement important!"33 » Alors que les femmes au foyer des années 1950 et 1960 freinaient leurs assignations par le biais de micro-résistances (vaudeville, petites piques, courtes modifications comportementales, usage modéré de la magie), Maude et Roseanne opèrent une résistance beaucoup plus frontale, où le sarcasme remplace les traits d'esprit et les comiques de situation.

Diriger ainsi les projecteurs vers la sphère domestique permet de s'attacher à décrire les impasses patriarcales qui résistent aux femmes malgré les avancées féministes. Les luttes prennent corps dans ces liens entre les personnages, plus précisément entre des mères ouvertement féministes et, non pas un personnage en particulier, mais tout un ensemble de comportements et de codes auxquels leurs proches les confrontent, souvent sans réaliser qu'ils écrasent leur mère ou leur épouse sous le poids de leurs allants-de-soi. Maude et Roseanne proposent de nouvelles figures maternelles dont les airs matriarcaux révèlent par endroits les peurs et les angoisses de la montée du féminisme. Ce n'est pas un hasard si les contestations les plus virulentes émanent des personnages évoluant avant tout dans la sphère domestique. La vigueur de leurs revendications et leur féminisme explicite encouragent l'interprétation de ces personnages comme avatars des contre-publics subalternes de la révolution sexuelle et de ses suites. Dans ces séries aux allures plus radicales que libérales, la maison se charge des politiques familiales, amoureuses et féministes : les héroïnes, contestataires, « constituent des arènes discursives parallèles dans lesquelles [elles] élaborent et diffusent des contre-discours, afin de formuler leur propre interprétation de leurs identités, leurs intérêts et leurs besoins ${ }^{34} \gg$. La construction se fait jour de ce que l'on peut appeler une «sphère publique intime » où les amoureux, à l'instar des citoyens étudiés par Nancy Fraser, « débattent de leurs affaires communes ».

Le modèle de l'injustice sociale de Nancy Fraser aide à déchiffrer les envies et les espoirs d'héroïnes qui ne dissocient plus les revendications féministes de leurs désirs amoureux. Pour Nancy Fraser, l'injustice sociale et les dénis de reconnaissance se logent non pas dans des interactions psychologiques, comme le défend Axel Honneth, mais dans des structures socioéconomiques et peuvent être résolus par une transformation de leurs deux pôles d'exercice que sont la redistribution (économique) et la reconnaissance (symbolique). La distinction est en réalité analytique, souligne 
Fraser, en ce que ces deux territoires se superposent et s'incitent l'un l'autre. Les séries télévisées ont très tôt pris acte de ces pôles lorsque, nous l'avons vu, elles représentent des femmes gestionnaires mais jamais rétribuées, soupirant de n'être pas reconnues pour leur travail domestique et éducatif. Un tournant opère cependant avec les héroïnes des décennies 1970-1980 : les injustices se font plus saillantes dans ces fictions qui, produisant deux grands types d'héroïnes (femmes actives et féministes au foyer) mettent l'accent tour à tour sur les problèmes de redistribution et d'indépendance économique et leurs répercussions sur la vie amoureuse (ce sont les femmes actives) ou sur les assignations domestiques comme entraves à la reconnaissance, y compris conjugale (ce sont les féministes au foyer). Cette difficulté à s'adapter provient en grande partie du déni de reconnaissance de la participation aux définitions des modalités relationnelles. Les hérö̈nes affrontent des difficultés à être entendue d'égale à égal dans la construction des relations amoureuses, révélant aux problèmes de démocratie du couple des racines de reconnaissance individualiste.

\section{Les revendications contradictoires de Maude}

Du haut de son mètre quatre-vingt, avec sa voix rauque, son langage corporel peu gracieux mais assuré et ses dialogues acérés loin du devoir et de l'oblat maternels, Maude est l'aïeule des féminités masculines de Murphy Brown et de Roseanne, tant et si bien que pour Bonnie Dow, « si Mary Richards était la figure fantasmée du féminisme », du fait de sa féminité assurée et rassurante, « Maude en était son cauchemar ${ }^{35}$. » Issue de la classe

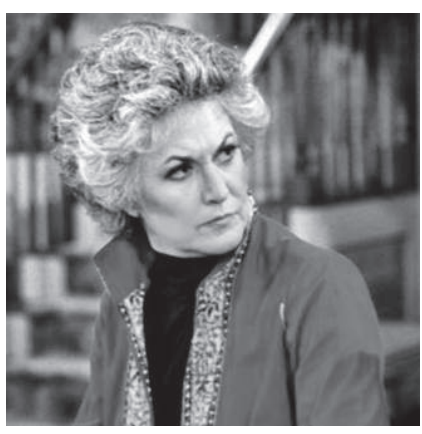
moyenne, âgée de quarante-sept ans au début de la série qui compte six saisons, la cartographie relationnelle de Maude témoigne déjà en 1972 des délitements amoureux puisque l'héroïne, mariée à son quatrième époux, vit avec sa fille divorcée. La série est traversée des contradictions entre les discours et les pratiques : au-delà de ses propos explicitement féministes, Maude est pendant de nombreux épisodes une femme au foyer dont l'époux lui interdit de travailler. Maude a été particulièrement encensée dans les recherches sur les héroïnes féministes du fait de sa prise de position explicite 
en faveur du féminisme, et qui plus est en faveur d'un féminisme à la teneur plus radicale que libérale, en même temps qu'a été mise en lumière la protection que lui offre sa position sociale relativement privilégiée. Bonnie Dow et Susan Douglas soulignent à raison le « côté pile » de la facette subversive et braillarde de Maude : l'héroïne est à l'abri des problèmes financiers grâce à son époux et peut ainsi aborder les sujets féministes dans une perspective purement socioculturelle, sans se préoccuper des conditions matérielles et de leurs conséquences. La monstration du féminisme radical, courant largement absent des imaginaires médiatiques et balayé par la mouvance libérale dans la sphère publique politique, semble ne pouvoir se faire qu'à la condition de son inscription dans une classe sociale favorisée, comme si le féminisme radical se chargeait des égalités de fait (acter le droit d'avorter, affirmer sa subjectivité, ne pas se conformer aux codes traditionnels de la féminité) pendant que le féminisme libéral s'occupe des égalités de droit (ainsi Mary Richards accédant au travail). Douglas elle-même rend réversible son herméneutique puisqu'elle considère successivement la féminité puis la masculinité comme la nécessaire concession pour montrer une héroïne aux prises avec des problèmes féministes lorsque tour à tour, elle envisage la douceur de Mary Richards puis la pugnacité de Maude comme des prix à payer au patriarcat et non comme des compromis représentationnels. Il y a là un cadre interprétatif simplificateur dans lequel les héroïnes semblent de toute façon toujours perdantes face à des normes rendues surplombantes : si elles sont féminines, elles stéréotypent les femmes; si elles sont masculines, elles stéréotypent les féministes. Le concept de norme lui-même devient problématique lorsqu'il est érigé en sésame interprétatif, prisme souverain par lequel toute représentation devient commodément univoque, quitte à aplatir toutes les contradictions pour que le matériau entre dans le cadre préconstruit. La norme n'est plus prescription, elle n'est même plus injonction (ce qui supposerait résistance) : elle redevient habitus, deuxième nature de personnages pourtant imaginaires que le chercheur entend démystifier (et par là même, bien sûr, le public).

Maude se veut médiatrice des combats féministes radicaux, mais aussi des perceptions hétéroclites du monde qui perdurent à toute révolution, qu'elles soient de genre, de classe ou même de race. Les interactions entre Maude et sa femme de ménage noire Florida sont le lieu d'affrontements idéologiques entre une femme blanche aisée et une femme noire populaire, 
la première essayant bien souvent de sauver la seconde malgré elle, tant et si bien que Florida veut démissionner, exaspérée des injonctions libératrices de Maude qui tente de lui imposer une solidarité de genre et de classe de principe, à rebours de ses propres intérêts ${ }^{36}$. La catégorie sociale de Maude permet de traiter le caractère parfois hégémonique d'un mouvement contre-hégémonique comme le féminisme, en mobilisant ce que par anachronisme l'on pourrait appeler l'intersectionnalité mais qui renvoie plutôt à l'importance grandissante du black feminism dans la sphère publique et dans les imaginaires médiatiques (deux ans plus tard, Get Christie Love! mettra en scène une policière afro-américaine).

\section{Roseanne: Les amours en milieu ouvrier}

Point de Noirs-A méricains ou de variables d'âge dans Roseanne, qui s'attache précisément à représenter le quotidien d'une famille ouvrière blanche. En réponse aux parfaites femmes au foyer des années 1950, Roseanne est le signe en 1988 d'un fort désenchantement : les personnages sont des Étasuniens de classe populaire, mariés à la sortie du lycée, peu diplômés et obligés de cumuler les emplois pour payer les factures. La famille Connor forme le cœur de la série la plus axée sur les problématiques de classe, au prisme duquel sont traitées les questions d'émancipation féminine et d'amour et au crible duquel passe le féminisme de la deuxième vague. Prise au piège de sa situation socioéconomique, prisonnière de sa maison sous emprunt et de ses dettes, Roseanne tente d'échapper à l'aliénation du quotidien domestique mais n'a pas le luxe de trouver l'émancipation dans la sphère publique comme les femmes diplômées. Quand elle travaille, elle est stressée et manque de temps; quand elle est sans emploi, elle angoisse et manque d'argent. Roseanne se veut porte-parole des oubliées des mouvements féministes américains de la deuxième vague qui dans leurs tonalités matérialistes promeuvent la reconnaissance du travail domestique des femmes au foyer ou la conquête de la sphère publique comme chemin de l'émancipation mais négligent le quotidien des Américaines de classe ouvrière.

Ni June Cleaver ni Murphy Brown, l'hérö̈ne ouvrière témoigne du décalage entre les promesses du bonheur familial de la première modernité et la concrétude des quotidiens laborieux pour des femmes que le contexte socioéconomique américain oblige à cumuler les emplois et à tenir des 
budgets serrés. Roseanne est originale dans ses articulations entre classe et genre (la race étant reléguée à la blanchité invisibilisée de cette famille moyenne) : ce n'est pas le patriarcat qui lui refuse l'accès à une profession épanouissante mais ses manques de compétences dues à son origine sociale, ce qui renverse radicalement l'ordre patriarcal du couple et de la famille. Le rêve américain est mort, en même temps que la dichotomie privé/ féminin/non-rémunéré versus public/masculin/rémunéré. Roseanne, de nombreuses fois, se targue d'être celle qui ramène le pain à la maison. Enregistrant une vidéo à destination de son futur enfant, son monologue mérite d'être cité dans la longueur :

Roseanne : - Ton père travaille très dur [...] mais je dois quand même dire que peu importe à quel point il travaillait dur, j'ai toujours travaillé encore plus dur que lui. J'ai travaillé qu'il soit embauché ou licencié. C'est moi qui ramenais le bacon à la maison, c'est moi qui le cuisinais. Alors ok, d'accord, j'en ai mangé un bout de temps en temps, mais je me disais que je devais bien me récompenser d'une manière ou d'une autre. Je ne me suis jamais plainte de toute ma vie. Je ne me suis jamais plainte et je n'ai jamais demandé de l'aide à quiconque. Parce que, tu sais, même si je l'avais fait, ce n'est pas comme si quelqu'un aurait bougé le petit doigt de toute façon ${ }^{37}$.

Cumulant les emplois quand Dan peine à en trouver un, Roseanne n'est pas l'employée du patriarche à l'instar de Samantha Stevens mais une cheffe de famille qui pense ne pouvoir compter que sur elle-même. Ses observations et demandes appellent peu de réponses et quand sa sœur ne manque pas de souligner qu'elle est autocratique, Roseanne s'en amuse : « Je ne régente pas Dan, j'essaie de le mettre en contact avec son côté soumis ${ }^{38}$. »Ainsi, la résolution des conflits réside le plus souvent dans l'abandon d'une des deux parties (en écrasante majorité, celle que défend Dan) que dans le compromis. L'articulation de la classe ouvrière de Roseanne avec son féminisme rend ses discussions démocratiques car franches et honnêtes, en accord avec les imaginaires de la classe populaire, mais elle transforme ses décisions en pouvoir autocratique qui, contrairement à l'arbitraire du patriarcat, est légitimé par le travail. Dans l'histoire 
des héroïnes télévisuelles, Roseanne fait ainsi figure de vilain petit canard : brune, grosse, bruyante, rustique, pauvre, elle est une « femme indisciplinée ${ }^{39} \gg$, selon l'expression de Kathleen Rowe, dont les constantes transgressions de la bienséance servent à rendre ces imaginaires visibles, drôles et positifs de façon si contrôlée qu'elle devient mascarade. Sa corpulence, sa voix nasale, ses cris, son appétit pantagruélique rappellent le corps grotesque de Bakhtine, « ce corps ouvert [qui] n'est pas franchement délimité du monde, [qui] est cosmique, [qui] représente et incarne tout l'univers matériel et corporel, compris comme le bas absolu ${ }^{40} \gg$.

Le corps déborde. Il déborde les marges matérielles mais aussi comportementales et relationnelles, jusqu'à atteindre un carnavalesque qui, à défaut de subvertir les hiérarchies comme a pu le défendre Bakhtine, les rend visibles. Pour Eco en effet, « Le carnaval, pour être apprécié, requiert que les règles et les rituels soient parodiés, et que ces règles et ces rituels soient déjà reconnus et respectés. L'on doit savoir à quel point ces comportements sont interdits, et l'on doit ressentir la majesté de la norme prohibitionniste, pour apprécier leur transgression ${ }^{41}$. $\gg$ Rowe a raison de dire que pour Roseanne, plus foucaldienne que freudienne, le pouvoir visuel est diffus et peut être réapproprié par les femmes elles-mêmes pour rendre visibles ce qui ne l'était pas ${ }^{42}$. À la façon des clowneries de Lucy Ricardo, Roseanne semble arracher sa subjectivité en dénaturalisant les biopolitiques de la femme au foyer. Mais le carnavalesque n'est pas que corporel, il est également communicationnel. La relation de Roseanne et

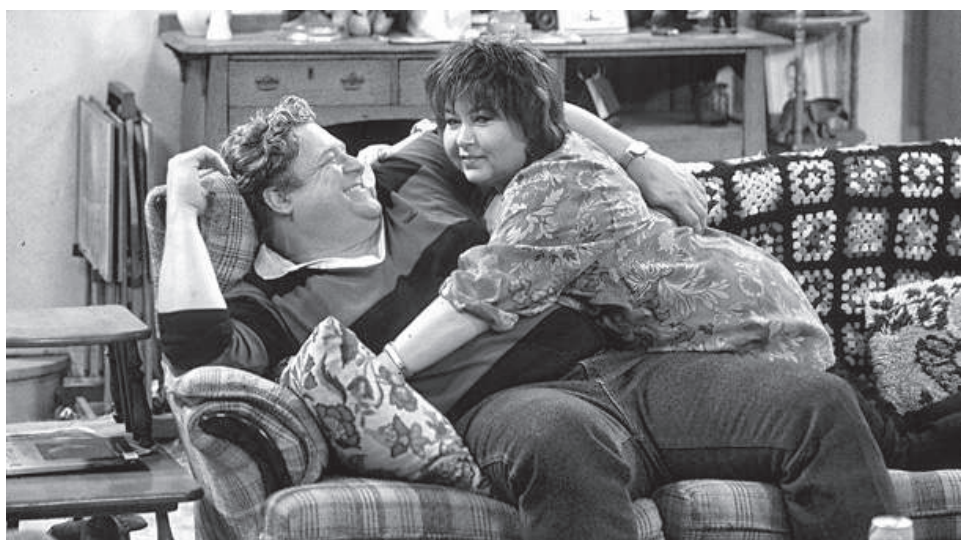


Dan est dans la démesure : dans leurs disputes, les tons montent, les gestes sont exagérés, les sujets sabordés frontalement - même ceux jugés grossiers comme lorsque Roseanne rappelle à Dan qu'enceinte, elle seule subira les nausées, les douleurs, les hémorroïdes, les couches et l'allaitement. Elle est finalement une héroïne hoggartienne qui exalte les qualités du populaire et qui s'insère directement dans les imaginaires des classes ouvrières tels que les décrit le sociologue britannique : au-delà de leurs caractéristiques individuelles qui sont « un parler piquant, une verve, teintée d'humour, qui ne se départit jamais d'un vigoureux bon sens », les gens du peuple sont vus comme se référant « à des qualités concrètes telles la gentillesse, la droiture, la franchise et la simplicité dans les relations humaines ${ }^{43} \gg$. Finalement, Roseanne ironise pour pouvoir être franche et vit une « vie dense et concrète, où l'accent est mis sur le sens de l'intimité, la valeur du groupe domestique et le goût des plaisirs immédiats ${ }^{44} \gg$. Elle est donc brune, grosse, bruyante, rustique et pauvre, mais elle est aussi déterminée, attentionnée, ouverte, simple et honnête. Ces valeurs populaires sont exacerbées dans le mariage de Dan et Roseanne dont la clé de voûte n'est pas la romance, auquel plutôt il s'oppose, mais la franchise et la confiance qui doivent permettre au couple de survivre aux frustrations de la vie commune et de la difficulté de partager un espace.

\section{Désenchantements quotidiens et microphysique des plaisirs}

Une grande originalité de ces deux séries est l'attention qu'elles confèrent à la vie quotidienne des amours, trivialité souvent disqualifiée. En même temps qu'elles regrettent le manque de romantisme (« ta conception du romantisme, c'est d'ouvrir ta cannette loin de mon visage », reproche Roseanne à son époux), les héroïnes envisagent la vie quotidienne comme l'épreuve et la preuve du couple. Après avoir, lors d'une dispute, brisé toute la vaisselle et ruiné le dîner, Maude et Walter se réconcilient et soulignent que « l'amour, c'est manger des sandwiches au thon dans des assiettes en carton $^{45} \gg:$ la présence et l'affection du partenaire suffisent à transcender de vulgaires sandwiches. L'amour se construit ainsi dans l'acceptation des défauts d'autrui et dans la simplicité qu'il vient embellir. Néanmoins, si Maude et Roseanne dévoilent un désenchantement de l'amour par la vie 
quotidienne, c'est pourtant en cette dernière qu'elles trouvent du sens à la construction du couple : Roseanne et Dan échangent, tout au long de la série, des sourires très complices, signifiant une intimité au-delà du verbal produite sur l'engagement dans une « agapè conjugale », selon l'expression de Jean-Claude Kaufmann, une agapè « enrichie de confiance et de reconnaissance réciproques ${ }^{46} \gg$. Le confort et la prospérité exaltés dans les précédentes décennies sont balayés en même temps que sont brisés vaisselles, meubles, télévisions, cadres : l'amour ne se loge plus dans la concrétisation matérielle, laquelle serait obtenue financièrement par l'époux et entretenue par la femme au foyer, mais dans des relations qui ne sont pas mesurables. Dans cette prosaïque du couple, la poésie trouve sa place par une microphysique des plaisirs : «L'alchimie nouvelle consiste à transformer des poussières en brins d'or aux quatre coins du couple et de la maison, à ravir les sens par des parfums, des couleurs, des touchers caressants ${ }^{47}$. $\gg$ L'amour y est construit d'attention, de respect et de reconnaissance, les sarcasmes et reproches représentant, au-delà de l'exutoire qu'ils sont, l'honnêteté nécessaire à la confiance mutuelle.

Mais toutes ces séries, de Murphy Brown à Roseanne, se font également l'écho, et ce n'est pas là dire qu'elles les promeuvent, des angoisses vis-à-vis des conséquences du féminisme. Les risques d'une domination renversée planent sur ces représentations médiatiques. Dans Murphy Brown, l'héroïne apparaît comme butée et austère et le problème du sacrifice se transforme en un refus, par principe, de tout compromis : Murphy refuse catégoriquement de faire des sacrifices au prétexte que l'on ne les demanderait pas à un homme, mais elle ne réalise pas au passage que ses partenaires sont désormais prêts à en faire. Chat échaudé craint l'eau froide : les hérö̈nes ont peur de perdre le lourd travail qu'elles ont accompli pour la sphère professionnelle dans une relation romantique oblative. Le refus

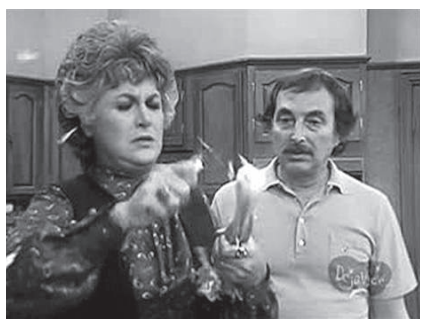
féminin de se sacrifier menace sans cesse de se transformer en affirmation vengeresse d'un pouvoir féminin débridé et castrateur. Les métaphores culinaires se poursuivent, à cela près que le pain phallique qui écrasait Lucy Ricardo contre le mur devient un céleri-branche (tout aussi phallique !) réduit en bouillie par Maude, et que le four asservissant est désormais un épluche-légumes châtreur : l'héroïne transforme en armes les outils domestiques jadis aliénants. 
Jusque dans les luttes internes à la production industrielle, les caricatures castratrices se font jour. Durant les premiers épisodes de sa série, Roseanne Barr a été dépossédée de sa création qu'ABC a confiée à un homme, Matt Williams. Barr est tout de même restée jusqu'à ce qu'un violent désaccord ne l'oppose à Williams. Cette querelle est significative des politiques de représentation des contre-publics subalternes féministes dans les années 1970-1980 : elle prenait source dans un dialogue que l'héroïne adresse à son époux et qui, selon Barr, « était une interprétation ridiculement sexiste de ce que pense une féministe - quelque chose du genre "tu es mon égal au lit, mais c'est tout”. [C’était] un dialogue castrateur que seul un abruti peut imaginer écrire pour une vraie femme qui aime son $\operatorname{mari}^{48} \gg$. Roseanne Barr fit grève le temps d'un épisode durant lequel elle vit le personnage de sa sœur la remplacer brillamment... dans les tâches domestiques - stratégie explicite de Matt Williams pour amorcer un remplacement. Soutenue par les acteurs principaux, Roseanne Barr réintègrera finalement la position de showrunner. De ce point de vue, la différence affirmée entre copyright américain et droits d'auteur français, si bien mise en évidence par Dominique Pasquier à propos des scénaristes de télévision ${ }^{49}$, peut être parfois plus floue dans un contexte opérationnel.

La remarquable absence de victimisation, que ce soit chez les personnages de femmes actives ou de féministes au foyer, encourage l'affirmation et le respect des subjectivités féminines. On voit bien que la participation à ce que l'on peut appeler la sphère publique intime n'est pas un corrolaire mécanique de l'égalité d'accès à la redistribution, c'est-à-dire à la participation à la sphère professionnelle. Tandis que les fictions des décennies 1950-1960 proposaient pour solution des remèdes que Nancy Fraser appelle « correctifs », c'est-à-dire qui « visent à corriger les résultats inéquitables de l'organisation sociale sans toucher à leurs causes profondes ${ }^{50} \gg$, Mary, Murphy, Maude et Roseanne se font plus «transformatrices » en ce qu'elles visent la « structure d'évaluation culturelle sous-jacente » à leur déni de reconnaissance : toutes tâchent de prendre conscience de ce qui participe, dans la construction de la féminité, à la position de subalterne qu'elles tentent de dépasser soit par des influences féministes plutôt libérales en demandant à gommer les différences (Mary et Murphy s'offusquent de se voir poser des questions qu'un homme ne subirait pas), soit de façon plus radicale en soulignant lesdites différences et les inégalités qu'elles 
produisent (Maude et Roseanne insistent sur le coût de leur identité féminine). Les injustices vécues par les héroïnes ne sont en tout cas plus résolues par l'apaisement de leurs symptômes à l'instar des attentions et remerciements, mais par l'accès à la sphère publique et à la reconnaissance sociale, dont on comprend désormais que le déficit provient aussi des modèles amoureux qui construisent le genre et l'identité subalterne. Le problème dans la redéfinition des contrats amoureux est similaire à celui soulevé par la redistribution et la reconnaissance, par le féminisme déconstructiviste et par le néoféminisme, de savoir s'il faut gommer les différences entre hommes et femmes pour parvenir à une égalité d'accès à la sphère publique intime ou s'il faut, au contraire, s'y attarder pour mieux rendre compte des inégalités présentes et passées. La tension entre l'universalisme et le (dé)constructivisme identitaire apparaît au cœur de la démocratie relationnelle à laquelle chacun doit en théorie avoir un accès égal mais qui, en pratique, est largement corrompu par les rapports de pouvoir genrés et amoureux qu'il s'agit de contrebalancer. 


\section{Notes}

1 Pour une discussion des choix définitionnels entre héros (féminin) et héroïne, voir l'ouvrage de Bilat, Loïse, Haver, Gianni, Le héros était une femme..., Paris, Antipodes, 2011. Les auteurs y montrent que le terme « héros » renvoie aux attributs mélioratifs de la masculinité (la force et le courage parmi d'autres) et désigne la place principale du récit alors que « l'héroïne est beaucoup plus floue sémantiquement ». Elle traîne encore sa définition classique, celle d'un personnage secondaire qui accompagne le héros masculin sans nécessairement accomplir d'exploits. Plus concrètement, si nous avons des difficultés à considérer Donald Duck comme un héros, « nous avons bien moins de peine à caractériser Martine comme une héroïne ». Je choisis ici de préserver le terme d'《 héroïne » qui, à condition de lui faire opérer un retournement de stigmate, traduit mieux les médiations féministes que représente l'émergence de personnages principaux féminins dans des États-Unis aux prises avec les questions d'égalité de genre.

2 Lehman, Katherine, Those Girls. Single Women in Sixties and Seventies Popular Culture, Austin, University of Kansas, 2011.

3 Dow, Bonnie J., Prime-Time Feminism. Television, Media Culture, and the Women's Movement Since 1970, Philadelphia, University of Pennsylvania Press, 1996.

4 Lehman, Katherine, ibid., p. 1.

5 Dow, Bonnie J., ibid., p. 28-29.

6 Lee, Janet, « Care to Join Me in an Upwardly Mobile Tango? Postmodernism and the "New Woman" », in Gammann, Lorraine, Marshment, Margaret, The Female Gaze. Women as Viewers of Popular Culture, St Paul, The Women's Press, 1988, p. 166-172.

7 Dow, Bonnie J., ibid., p. 73.

8 Gurley Brown, Helen, Sex and the Single Girl, New York, Bernard Geis Associates, 1962.

9 Ouellette, Laurie, « Inventing the Cosmo Girl. Class Identity and Girl-Style American Dreams », in Dines, Gall, Humez, Jean M., Gender, Race, and Class in Media: A Critical Reader, Londres, Sage Publications, 2011. 
10 Lehman, Katherine, Those Girls. Single Women in Sixties and Seventies Popular Culture, Austin, University of Kansas, 2011.

11 Taylor, Ella, Prime-Time Families: Television Culture in Postwar America, Oakland, University of California Press, 1989.

12 Dow, Bonnie J., ibid., p. xxiv.

13 Probyn, Elspeth, « New Traditionalism and PostFeminism: TV Does the Home », Screen, 31, 2, 1990, p. 147-159.

14 Macé, Éric, « Mouvements et contre-mouvements culturels dans la sphère publique et les médiacultures », in Maigret Éric, Macé, Éric, Penser les médiacultures. Nouvelles pratiques et nouvelles approches de la représentation du monde, Paris, Armand Colin, 2005.

15 L'image est devenue si célèbre que le 8 mai 2002, la chaîne de télévision TV Land en inaugura une statue de bronze.

16 « How will you make it on your own? This world is awfully big and girl this time your all alone but it's time you started living, it's time you let someone else do some giving. Love is all around, no need to fake it. You can have the town, why don't you take it. You're gonna make it after all, you're gonna make it after all. », Joan Jett and the Blackhearts, Love is all around.

17 The Mary Tyler Moore Show, saison 1, épisode 1.

18 The Mary Tyler Moore Show, saison 1, épisode 2.

19 The Mary Tyler Moore Show, saison 1, épisode 8.

20 The Mary Tyler Moore Show, saison 1, épisode 14.

21 The Mary Tyler Moore Show, saison 1, épisode 5.

22 Bathrick, Serafina, « The Mary Tyler Moore Show. Women at Home and at Work », in Morreale, Joanne, Critiquing the Sitcom. A Reader, Syracuse, Syracuse University Press, 2002, p. 160.

23 Newcomb, Horace, Alley, Robert S., The Producer's Medium. Conversations with Creators of American TV, Oxford, Oxford University Press, 1985, p. 219.

24 Gitlin, Todd, « Prime Time Ideology. The Hegemonic Process in Television Entertainment », Social Problems, 26, 1979, p. 251-266.

25 Fiske, John, « Murphy Brown, Dan Quayle, and the Family Row of the Year », in Media Matters: Everyday Culture and Political Change. Race and Gender in U.S. Politics, Minneapolis, University of Minnesota Press, 1996, p. 29. 
26 «e mariage est une question morale qui requiert un consensus culturel et l'usage de sanctions sociales. Mettre au monde un enfant de façon irresponsable est tout simplement mal. Manquer au soutien d'un enfant dont on est le père est mal. Il faut nous soyons sans équivoque à ce propos. Ça n'aide pas que la télévision de prime-time ait Murphy Brown, un personnage qui est supposé incarner les femmes intelligentes, très bien payées et professionnelles d'aujourd'hui, moquer l'importance du père, mettre seule un enfant au monde et appeler ça juste un autre "choix de vie". Je sais que ce n'est pas à la mode de parler des valeurs morales, mais nous devons le faire. Même si nos leaders culturels à Hollywood, dans les networks télévisuels, dans les quotidiens nationaux les raillent quotidiennement, je pense que la plupart d'entre nous ici savent que certaines choses sont bonnes, et que d'autres choses sont mauvaises. Il est temps de rendre cette discussion publique. Il est temps de parler à nouveau de la famille, du labeur, de l'intégrité et de la responsabilité individuelle. Nous ne pouvons pas être embarrassés par notre croyance en ce que deux parents, mariés l'un à l'autre, sont dans la plupart des cas meilleurs pour des enfants qu'un seul parent. » Extrait du discours de Dan Quayle au Commonwealth Club of California, San Francisco, 19 mai 1992.

27 Sur le traitement médiatique de Murphy Brown versus Dan Quayle, nous renvoyons à l'excellent article de John Fiske pour ne garder ici que les occurrences les plus percutantes : Fiske, John, « Murphy Brown, Dan Quayle, and the Family Row of the Year », in Media Matters: Everyday Culture and Political Change. Race and Gender in U.S. Politics, Minneapolis, University of Minnesota Press, 1996.

28 «Quayle says television contributed to the L.A. riots, says "Murphy Brown" mocked the importance of marriage », Los Angeles Times, 20 mai 1992.

29 Murphy Brown, saison 4, épisode 16.

30 «United States of Television. America in Prime Time. Episode 1 : The Independent Woman », PBS, 2011.

31 The Mary Tyler Moore Show, saison 1, épisode 1.

32 Roseanne, saison 1, épisode 1.

33 «United States of Television. America in Prime Time. Episode 1: The Independent Woman », PBS, 2011. 
34 Fraser, Nancy, « Repenser la sphère publique : contribution à la critique de la démocratie telle qu'elle existe réellement », Hermès, 31, 2001, p. 125-157.

35 Dow, Bonnie J., Prime-Time Feminism. Television, Media Culture, and the Women's Movement Since 1970, Philadelphia, University of Pennsylvania Press, 1996, p. 61.

36 Maude, saison 1, épisode 3. Voir également Maude, saison 2, épisode 6.

37 Roseanne, saison 8, épisode 10.

38 Roseanne, saison 7, épisode 20.

39 Rowe, Kathleen, « Roseanne: Unruly Woman as Domestic Goddess », Screen, 31, 1990, p. 408-410.

40 Bakhtine, Mikhaïl, L'ouvre de François Rabelais et la culture populaire au Moyen-Age et sous la Renaissance, Paris, Gallimard, 1970, p. 36.

41 Eco, Umberto, « The Frames of Comic "Freedom” », in Eco, Umberto, Ivanov, V.V., Recto, Monica, Carnival!, Berlin, De Gruyter, 1984, p. 6.

42 Rowe, Kathleen, ibid., p. 408-410.

43 Hoggart, Richard, La Culture du pauvre. Étude sur le style de vie des classes populaires en Angleterre, Paris, Les Éditions de Minuit, 1970, p. 152 .

44 Hoggart, Richard, ibid., p. 151.

45 Maude, saison 1, épisode 7.

46 Kaufmann, Jean-Claude, L'Étrange histoire de l'amour heureux, Paris, Fayard, 2010, p. 153.

47 Kaufmann, Jean-Claude, ibid., p. 154.

48 Barr, Roseanne, «And I Should Know », New York Magazine, 15 mai 2011.

49 Pasquier, Dominique, Les scénaristes et la télévision. Approche sociologique, Paris, Nathan Université, 1999.

50 Fraser, Nancy, ibid., p. 31. 
Les contradictions des héroïnes de l'après-féminisme (1997-2004) 



\section{Expressivisme, post-modernité et après-féminisme}

\section{De « nouvelles "nouvelles femmes" » aux identités sensibles}

Au milieu des années 1990, sous l'impulsion d'une troisième vague féministe qui s'attache à déconstruire le genre et à intégrer les thématiques de diversité, la télévision étasunienne opère un tournant en proposant massivement des héroïnes héritières des avancées fragiles de la deuxième vague. À partir des années 1990, ces personnages prennent un tournant réflexif: les femmes représentées sont supposément détachées des structures traditionnelles et envisagent leurs choix comme des projets de vie soumis à la question du bonheur et de l'épanouissement personnel. En résulte une tension entre l'accomplissement des idéaux professionnels de la deuxième vague et son prix affectif, les deux sphères publique et privée n'étant plus imperméables mais restant mutuellement exclusives, ce qui amène Amanda Lotz à les qualifier de «nouvelles "nouvelles femmes" », à la suite des «nouvelles femmes ${ }^{1} \gg$.

Cet éboulement des structures, qui libère ou qui parfois coince les héroïnes, donne une tonalité postmoderne aux récits. Les personnages antérieurs, de la période 1970-1997, avaient des identités figées, au sens où elles ne changeaient presque jamais de métier ni d'environnement professionnel et où leurs relations amicales et familiales étaient stables et sécurisantes. C'est moins le cas à partir des années 1990 : les identités deviennent multiples et concurrentielles, ce qui complexifie les relations sociales. Cela se traduit par un allongement substantiel des récits. Alors que Mary Richards ne revoit presque jamais les hommes avec qui elle passe une soirée ou que 
Murphy Brown démêle en un seul épisode les deux demandes en mariage qu'elle reçoit (!), les « nouvelles “nouvelles femmes” » étirent leurs histoires amoureuses sur plusieurs épisodes, plusieurs saisons, voire sur toute la série. Les problèmes épisodiques se transforment donc en arcs narratifs, en articulation avec le développement d'une réflexivité qui nécessite du temps et des échanges pour se construire et pour résoudre les problèmes qui se posent à elle. L'affaiblissement de la modernité n'y est pas étranger. Pour Rosi Braidotti, la « crise du sujet moderne - l'Homme [fait que] l'altérité comme axe porteur du sujet est déstabilisée », « cette errance entraîn[ant] le sujet dans un mouvement de déterritorialisation ${ }^{2} \gg$. La voix donnée à ces autres féminins - des féminins hétérogènes - est évidente à partir des années 1990, de surcroît dans des contextes où « il n'est plus souhaitable ni suffisant de penser par oppositions dialectiques et dualismes structurants » comme c'était le cas dans la modernité.

En conséquence, les pratiques et les discours changent de registre en même temps qu' ils perdent de leur assurance. Les longs échanges conversationnels d'Ally McBeal ou la voix off interrogative, quasi introspective, de Sex and the City sont les signes de trajectoires désormais incertaines, de relations et d'identités en constante construction et reconstruction pour des héroïnes font montre d'interrogations, d'évolutions, de positionnements, de choix et de tactiques relationnelles - « polylogues avancés pour sujets nomades en transformation $^{3} \gg$. Cette réflexivité induit une anxiété que des béquilles psychologiques viennent pallier, prenant la forme de chansons fétiches (Ally McBeal), de surconsommation d'imaginaires médiaculturels (Gilmore Girls), de journaux intimes et de techniques d'introspection (Sex and the City), de yoga et de méditation (Dharma \& Greg, Sex and the City) ou de vibromasseurs et autres jouets sexuels (Sex and the City, Ally McBeal).

Les compétences émotionnelles sont de surcroît revalorisées. Autrefois étouffées par les structures familiales (une bonne mère est un individu qui se dit sacrifié) ou par les à priori genrés (une femme active est une femme forte), les émotions trouvent un lieu d'expression privilégié dans la réflexivité. Les femmes actives et les féministes au foyer luttaient pour des égalités de droit (accès à la sphère publique, demandes de reconnaissance, dépassement des doubles journées et doubles standards), tandis que les trentenaires, plus sensibles, s'attachent aux égalités de fait (égalité amoureuse, refus de la dépendance émotionnelle, problématique du bonheur). 


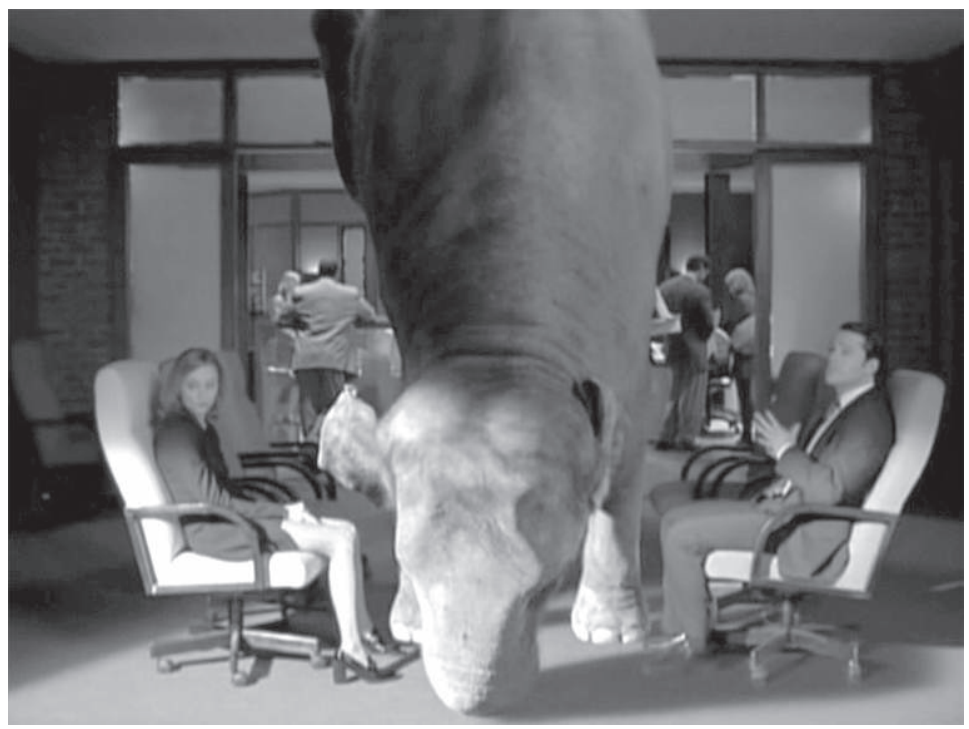

Ally McBeal a proposé des représentations originales de ces émotions en les matérialisant via des effets spéciaux permettant de faire blanchir ou rougir le visage d'Ally suite à une situation gênante, ou de remplacer une table séparant deux anciens amants par un éléphant, symbolisant l'embarras des amoureux qui, détournant le regard des acrobaties du pachyderme, ne veulent pas affronter leurs sentiments. Ce style visant à rendre visuellement littéraux des rêves, des fantasmes ou des expressions semble d'ailleurs se propager depuis peu aux représentations du masculin, que ce soit au cinéma (La Vie Rêvée de Walter Mitty) ou à la télévision (Man Seeking Woman). Là aussi, la concrétisation matérielle du monde imaginaire, qui est parfois une fuite dans l'allégorie, traduit une sensibilité accrue, qui est désormais également celle du masculin.

Mary Richards et Murphy Brown avaient certes enclenché le processus individualiste mais les discussions qui prenaient place dans ces séries concernaient rarement leur intimité affective. Parce que les compétences émotionnelles sont historiquement liées à la gestion du foyer familial, elles étaient, pour les femmes actives, la menace d'une assignation à la sphère privée. Or, à partir des années 1990, les héroïnes ont suffisamment sécurisé la sphère professionnelle et ne songent pas un instant à l'abandonner, 
ce qui les libère davantage du piège de la domination symbolique et leur permet de réinvestir ces caractéristiques historiquement féminines. Lorsque l'intime vient systématiquement structurer les récits, les personnages et leurs histoires se font ainsi plus expressivistes et émotionnelles, en même temps qu'elles sont plus stratégiques et politiques, une contradiction que Jean-Claude Kaufmann identifiait déjà lorsqu'il écrivait que, alors que « l'expression émotionnelle et sentimentale ne cesse de s'amplifier, le modèle de l'individu froidement calculateur n'a jamais été aussi prégnant au centre de la société, et va même jusqu'à s'insinuer dans les domaines les plus intimes ${ }^{4} \gg$. Les marges s'expriment, révélant des subjectivités protéiformes. Qu'elles soient loufoques, libérées ou profondément névrosées, les héroïnes interrogent les effets anxiogènes de la libération individuelle et font apparaître des identités au tissu stratégique et positionnel. Rosi Braidotti stipule que « nos façons d'habiter les paradoxes et les contradictions de la post-modernité tardive nous obligent plutôt à nous engager dans un dialogue en zigzag avec de multiples interlocuteurs à la fois ${ }^{5} \gg$ : la complexité de ces « nouvelles "nouvelles femmes" », qui a trop souvent été réduite à un antiféminisme, provient en fait, non seulement des stratégies industrielles (le syncrétisme des médiacultures), mais aussi de l'ambiguïté postmoderne (ou, du moins, de l'après-modernité) qui ne se satisfait plus des logiques dichotomiques. Réflexivité, marques humoristiques, introspections sont en fait les signes d'un sens en apparence plus flottant.

\section{La re-féminisation des héroïnes: néoféminisme ou postféminisme?}

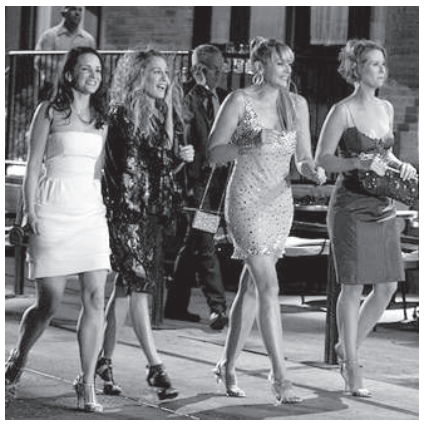

La féminité des héroïnes a, pour beaucoup d'auteur.e.s critiques, marqué une forme de backlash à partir des années 1990. Les balades citadines des héroïnes de Sex and the City s'inspirent explicitement des défilés de mode et, lorsque Buffy tue le vampire en talons hauts ou que les sorcières de Charmed chassent le démon courtement vêtues, l'invraisemblance de la performance n'est pas sans rappeler celle de Donna Reed qui passait l'aspirateur en robe bouffante, la taille fermement serrée par un ruban. Le consumérisme de ces séries est évidemment à rattacher au nouveau 
souffle capitaliste s'exprimant depuis la chute du Mur, à peine une décennie plus tôt : pour Jane Arthurs, «Sex and the City exploite l'approche consumériste des magazines féministes de la sexualité, dans lesquels le plaisir et l'agir sexuel féminin sont encouragés, car relevant du style de vie de la consommatrice. ${ }^{6}$ » Le consumérisme, qui était déjà étroitement lié à la fonction maternelle dans les années 1950, joue à nouveau un rôle crucial, reformulé à l'aune de la deuxième modernité : désormais individuel (les femmes disposent à leur guise de leurs revenus d'activité), il est individualisant puisque les achats ne sont plus ceux de la gestionnaire du foyer (robots ménagers, réserves alimentaires), mais viennent souligner l'accomplissement personnel. L'impressionnante collection de chaussures de luxe de Carrie Bradshaw et les tailleurs impeccables d'Ally McBeal sont les signes ostensibles de leur réussite professionnelle. En ce sens, le néoféminisme reste le privilège d'un groupe social bien particulier : celui des femmes jeunes, blanches et surtout de classe aisée.

Cependant, aussi évidente que soit la teneur traditionnelle de ces performances, la seule analyse des caractéristiques féminines et masculines s'avère limitée pour éclairer les positionnements stratégiques de ces identités. Difficile d'ignorer que, dans un mouvement d'émancipation à priori contradictoire, les héroïnes choisissent d'être si féminines : étant plus souvent dans l'auto-objectivation que dans l'objectivation simple, elles se disent conscientes de vouloir attirer l'attention d'autrui et utilisent leur féminité pour séduire. Pour bien saisir les enjeux féministes à l'œuvre dans ces féminités, il faut comprendre ensemble les discours, les arcs narratifs et les performances corporelles. Les résultats montrent des féminités en partie classiques (composées de cheveux longs, maquillage, bijoux, robes et jupes, décolletés...) mais également teintées de deux influences féministes : le néoféminisme puisqu'elles revalorisent le charme et la sexualité comme des atouts typiquement féminins; et le postféminisme car elles sont individualistes, consuméristes et stratégiques. Apparaît un réinvestissement nouveau du genre féminin basé sur l'exagération. Cette dernière peut relever d'une performance (c'est le cas des tenues haute couture de Carrie Bradshaw), du carnavalesque et du stratégique (les déguisements de Sydney Bristow), de l'ironie (la blondeur innocente de Buffy) voire du dramatique (la maigreur d'Ally McBeal a bien souvent été perçue comme un symptôme anorexique caractéristique de la fin des années 1990). 
Partout, des bribes de tradition et de première modernité créent des injonctions contradictoires comme la représentation d'un soi sophistiqué mais authentique. La mise à distance ironique est également présente chez les super-héroïnes qui chassent les monstres vêtues d'un pantalon de cuir ou combattent l'ennemi de la nation en usant de leurs charmes. Le renversement de stigmate est d'ailleurs assumé par le créateur de Buffy, Joss Whedon, qui a explicité dès le début sa volonté de conférer de l'empowerment à la figure de la femme sans défense :

La première chose à laquelle j'ai pensé quand j'ai créé le film Buffy, c'est cette fille blonde qui, dans tous les films d'horreur, va dans une allée sombre et se fait tuer. L'idée à l'origine de $B u f f y$, c'était de subvertir cette idée, cette image, et de créer quelqu'un qui, au lieu d'être la victime qu'elle a toujours été, devient une héroïne.

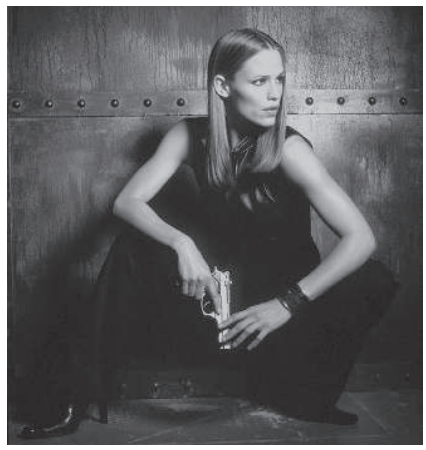

Dans Alias, les tenues que l'espionne Sydney Bristow revêt lors de ses missions sont des mascarades néoféministes qui exploitent l'objectivation comme la principale faille de l'adversaire masculin. Or, démaquillée et déshabillée de ces excentriques tenues, Sydney trouble le genre. Son visage angulaire, sa mâchoire saillante, son corps athlétique et notamment ses épaules très musclées suggèrent une masculinité que viennent accentuer le sérieux de ses tailleurs et la discrétion voire l'absence d'artifices féminins (coiffure, maquillage, bijoux).

\section{Tout avoir toute seule, le défi des héroïnes trentenaires}

Trois points interdépendants structurent finalement la représentation de cet héritage de la deuxième vague féministe. Le premier montre les succès féminins : professionnellement épanouies, financièrement indépendantes, sexuellement libérées et socialement stratégiques, les femmes représentées sont des sujets autonomes et responsables. Le second point rappelle les inégalités persistantes, de l'idéologie sexiste plus ou moins latente aux 
reproductions concrètes des rapports de domination. Le troisième, conséquence de la confrontation des deux premiers, est en partie un constat d'échec puisqu'il marque le décalage entre les revendications de la deuxième vague et l'environnement socioculturel contemporain : les héroïnes ont beau être professionnellement épanouies et ne pas céder aux menaces sexistes, elles n'en sont pas pour autant heureuses. Les femmes veulent faire tomber les frontières public/privé et briser les plafonds de verre mais elles se heurtent à la difficulté de « tout avoir » (《 to have it all ») comme le scandait le féminisme dans les années 1970. Si Ally McBeal s’écrie, « je veux du sexe, je veux une maison avec plein de meubles, je veux un bébé, je veux tout avoir $!^{8}$ »; si Carrie Bradshaw s'interroge, «sommes-nous submergées ou pouvons-nous tout avoir ?" »; si enfin Lorelai Gilmore avoue, «j'ai l'impression que je n'aurai jamais la totale ${ }^{10} »$, c'est parce que les promesses féministes sont en crise, amenant les hérö̈nes à faire le bilan du féminisme.

Les mutations qui ont eu lieu au niveau de l'individu (notamment en termes de liberté professionnelle, sentimentale et sexuelle) peinent à se traduire dans les rapports avec les hommes. Ceux-ci restent fermement ancrés dans des idéologies patriarcales, voire y sont enfermés par des attentes féminines qui peinent à saisir le sexisme latent de certains profils - le Prince charmant ou le bad boy que recherchent les héroïnes sont des identités historiquement virilistes. Les déséquilibres entre hommes et femmes proviennent majoritairement de l'inégale répartition des charges mentales : les personnages féminins sont ceux qui portent le poids de la construction et de la réaffirmation des relations. De rares exceptions sèment un trouble dans le genre : des héroïnes comme Lorelai (Gilmore Girls), Samantha (Sex and the City) ou Max (Dark Angel) sont émotionnellement indépendantes et tergiversent rarement sur les intentions de leur partenaire, privilégiant les discussions franches. Mais plus souvent, les « nouvelles “nouvelles femmes” » ont une fragilité émotionnelle qui induit une hégémonie masculine. Cela s'explique conjointement par l'assignation historique des femmes à la sphère privée et par leur arrivée tardive dans la sphère publique. Par ricochet, leur participation à la démocratie relationnelle est alors structurée par des compétences émotionnelles fortes mais par une confiance en soi enrayée. Or, le cercle est vicieux : moins les hommes travaillent au maintien de la relation amoureuse, moins les femmes sont sûres d'elles, et plus elles font montre 
d'une forme de misère émotionnelle. Deux raisons expliquent ce déficit de confiance en soi : la première vient de la difficulté à élaborer des codes de séduction qui conviennent à leur nouvelle indépendance (les hommes prennent peur dès qu'elles évoquent leur autonomie). La seconde provient du labeur que nécessite désormais la construction de l'identité individuelle, laquelle risque d'être douloureusement remise en cause par le partenaire. Le lourd travail identitaire effectué par l'individu se trouve sans cesse menacé par l'évaluation interpersonnelle, et plus encore par l'évaluation amoureuse que le caractère intime rend très légitime. Or, pour affronter cette démultiplication des relations sociales, donc des foyers de reconnaissance, les femmes, longtemps contenues à la famille, sont historiquement moins armées que les hommes.

Le contrecoup est un surinvestissement du champ amoureux. Dans Sex and the City, Carrie est sans cesse l'instigatrice de discussions qu'elle veut si sérieuses qu'elles conduisent souvent à la dispute, sabotant ainsi sa relation avec $\mathrm{Mr}$ Big. Les sujets qu'elle aborde relèvent de la consolidation de la relation : publicisation de leur relation, exclusivité affective et sexuelle, degré d'attachement... autant de composantes qu'elle prend à sa seule charge, faute de pouvoir en discuter avec son partenaire. S'interrogeant par exemple sur les intentions de Big, Carrie s’enlise dans la surinterprétation : « Ça me fait me dire que tout ce qu'il m'a jamais dit et que j'avais interprété comme sincère est sujet à interprétation, et dans ce cas, ce que je perçois comme étant ses sentiments à mon égard ne sont peut-être que les projections de mes propres sentiments pour lui ${ }^{11}$. » L'absence de communication - sans même parler de sa structuration en régime démocratique - entérine l'ostracisme des femmes en matière de reconnaissance interpersonnelle, ce qu'elles interprètent comme la punition de leur émancipation.

Si les années 1990 signent l'apparition très explicite du mouvement féministe dans les représentations médiatiques, cela ne se fait qu'en l'assignant au féminin et en l'interdisant au masculin, hors du cadre militant ou activiste. Or, la question de l'appropriation du féminisme par les hommes devient cruciale. Certes, elle risque de plier en sens inverse le mouvement émancipateur mais, en retour, laisser le masculin au seul stade de l'objet de discussion du féminisme revient à laisser ce dernier à la seule charge du féminin et à entériner la déresponsabilisation des hommes, sans parler du fait que, comme le souligne Patrick Hopkins, l'idée que le féminisme est antinomique 
du masculin cache un essentialisme latent ${ }^{12}$. L'opposition putative entre hommes et féminisme repose en effet, comme le souligne Tom Digby, sur l'opposition binaire entre le genre masculin (qui serait consubstantiel à la domination masculine) et le genre féminin ${ }^{13}$. Sandra Harding, de son côté, insiste sur le potentiel subversif du féminisme radical, qui enjoint aux hommes de refuser l'assignation de genre ainsi que la hiérarchisation et la visée hégémonique qui en résulte : « les hommes peuvent refuser de respecter les idéaux de la masculinité qui structurent les cultures, les politiques et les pratiques de tant d'institutions sociales ${ }^{14} \gg-$ bref, ils peuvent produire des $\ll$ subjectivités post-patriarcales ${ }^{15} \gg$. Cela implique en retour d'interroger les statuts de sujet et d'objet du féminisme : faire des femmes l'unique sujet du féminisme aura été une étape cruciale de la construction du sexisme comme problème public, mais elle est une étape qu'il semble être temps de dépasser au risque de retomber dans de nouvelles formes d'essentialisme. L'une des plus dangereuses est celle du procès d'intention, qui consiste, de façon déterministe et réductionniste, à présupposer que la position sociale masculine induit mécaniquement telle ou telle position politique. Un tel projet a pour écueil d'exalter en retour les qualités des femmes du simple fait qu'elles sont dominées, ce qu'incarne le courant néoféministe. En retour, ouvrir le féminisme aux hommes ne relève pas d'un humanisme angéliste qui ignorerait les intérêts contradictoires des groupes sociaux, mais d'une stratégie politique de déconstruction des genres et de redistribution des pouvoirs. Aussi, ce qui pose problème dans le cas des personnages trentenaires n'est peut-être pas tant la sur-responsabilisation individualiste du féminin que la sous-responsabilisation féministe du masculin : si les femmes sont presque uniques garantes des relations amoureuses, la raison en incombe non pas à l'individualisme - qui tend plutôt à leur redonner ce dont elles ont été privées (la possibilité de s'épanouir par l'attention à soi et le temps personnel) - mais à l'assignation du féminisme aux femmes. La démocratisation des politiques féministes pour les hommes, et l'intégration de leur responsabilité dans la prévalence du romantisme, apparait comme une étape urgente pour amorcer la démocratie relationnelle.

Toutes ces reformulations font que les relations affectives débordent le seul champ amoureux, comme le résume Ulrich Beck : «Si seulement ce n'était qu'une question d'amour et de mariage... mais on ne peut plus définir les relations entre les sexes simplement en fonction de ce qu'elles 
semblent impliquer - la sexualité, l'affection, le mariage, la parenté, etc. - il faut inclure tout le reste, comme le travail, la profession, les inégalités, les politiques et l'économie ${ }^{16}$. » Face à ces résistances, les héroïnes semblent dépourvues d'outils politiques, ce qui n'entame pas leur détermination. Elles refusent de parlementer avec des personnages masculins qui se sentent menacés par leur épanouissement, disqualifiant immédiatement le potentiel intime de ces relations. Une telle opiniâtreté prévient toute lecture antiféministe (les héroïnes ne reviennent pas sur les acquis féministes), et éclaire, au contraire, les résistances sexistes auxquelles les femmes émancipées restent confrontées. Toutefois, en parallèle de cette conviction, les héroïnes se sentent coupables de tant vouloir une relation amoureuse, et ce malgré les modalités antisexistes qu'elles y exigent. Cette culpabilité provient de l'image qu'elles se font du féminisme : Lorelai Gilmore s'en veut de ne pas « être Wonder Woman » (autrement dit une super-femme); Ally McBeal explique que, étant « une femme forte et ambitieuse qui se sent vide sans un homme, la National Organisation for Women [a] un contrat sur [sa] tête $^{17} \gg$; enfin, Carrie, esseulée le jour de ses trente-cinq ans, culpabilise de vouloir un compagnon, « Je me déteste un peu de dire ça mais je me suis sentie vraiment triste de ne pas avoir un homme dans ma vie qui se soucie de moi, aucun homme spécial pour me souhaiter un bon anniversaire, pas une satanée âme sœur... et je ne sais même pas si je crois aux âmes sœurs $!^{18}$ » Les héroïnes se sentent indignes de l'héritage égalitariste car, en opposition supposément radicale avec les valeurs féministes, elles souhaitent pouvoir manifester ce qui va d'une vulnérabilité que compenserait le soutien d'un partenaire à une dépendance émotionnelle dans laquelle se perdre.

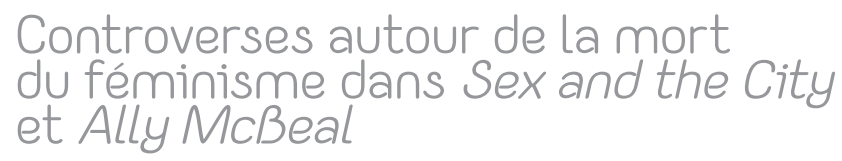

Parce que ces séries représentent la difficulté de cumuler épanouissement professionnel et personnel ainsi que le mal-être qui résulte de cette aporie, d'aucuns les ont interprétées comme un backlash punissant les émancipations. Pour Susan Faludi, les médias de masse condamnent le féminisme en semblant dire «Voyez comme elle est misérable, ce doit être parce que les femmes sont trop libérées $!^{19} \gg$ Des héroïnes professionnellement 
et émotionnellement épanouies seraient-elles trop menaçantes par leur empowerment? Narratologiquement trop pauvres ? Ou bien trop utopiques ? Analyser conjointement rapports amoureux et rapports de genre permet de réinscrire ces imaginaires dans ce que je propose d'appeler une crise $d u$ bonheurféministe, qui est à ne pas confondre avec le backlash. Là où le backlash prône le retour à la tradition, la crise du bonheur féministe interroge les impasses, les dangers et les désillusions. Les hérö̈nes invoquent en effet leur indépendance comme étant la cause de leurs déconvenues mais elles refusent catégoriquement de la remettre en cause, préférant attendre de rencontrer un partenaire suffisamment progressiste qui accepte leur émancipation.

Contre la conclusion faite par Susan Faludi d'une verve réactionnaire - malheureuses à cause de la sphère professionnelle, les femmes devraient retourner au foyer -, la montée de nouveaux espaces-temps (privé, public et leurs agencements temporels) et d'une forte problématisation des rapports amoureux et sexuels encourage une interprétation alternative. Que les « nouvelles “nouvelles femmes” » viennent d'abord de la sphère publique pour investir ensuite la sphère privée symbolise l'accomplissement du féminisme, tandis que leurs névroses et leurs malheurs somment de repenser le public et le privé ensemble et non l'un après l'autre.

Susan Faludi n'est toutefois pas la seule à avoir jeté l'opprobre sur ces représentations. Scientifiques, journalistes, acteurs de l'industrie télévisuelle et spectateurs ont produit des critiques si diverses et si prolifiques à l'égard de Sex and the City et d'Ally McBeal qu'une méta-analyse ne peut en tirer que la conclusion d'une ambiguïté typique des controverses autour de l'héritage du féminisme. Les représentations de ces séries dans les médias nous renseignent finalement presqu'autant que la profusion de discours apologiques ou dénonciateurs à leur égard.

Bon nombre de journalistes ont critiqué les liens entre ces « nouvelles “nouvelles femmes” » et le féminisme: Ginia Bellafante reproche dans un article du Timel'égocentrisme et le consumérisme des jeunes générations, titrant «Le féminisme : c'est moi qui compte ! » et sous-titrant «Vous voulez savoir ce qui intéresse les jeunes et chics penseuses féministes ? Leur corps ! Elles-mêmes $!^{20} \gg$. Le numéro est resté célèbre pour sa couverture qui aligne, en noir et blanc,

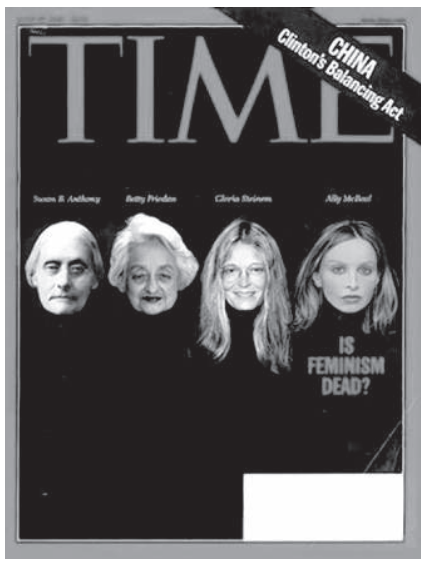




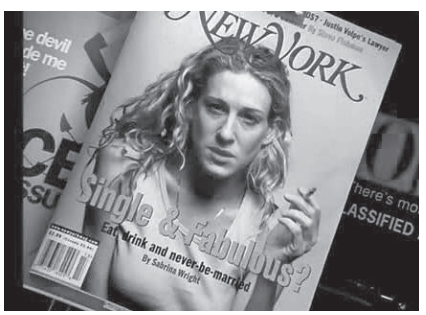

quelques grandes figures du féminisme (Susan B. Anthony, Betty Friedan, Gloria Steinem), suivies d'Ally McBeal, sous laquelle une phrase couleur sang demande « Le féminisme est-il mort ? » La critique des représentations médiatiques est ironiquement reprise par Sex and the City dans une pirouette réflexive, lorsque Carrie est piégée par un magazine qui, en lieu et place d'un portrait sophistiqué qui devait s'intituler « Seule \& Fabuleuse ! », publie une photo d'elle au réveil, plombée par une gueule de bois, remplaçant le point d'exclamation par un point d'interrogation. Pour Jane Arthurs, les analyses de Sex and the City la détachent trop souvent de son contexte, oubliant qu'elle a été parmi les premières à « répudier publiquement la honte d'être seule et sexuellement active au mépris des codes bourgeois ${ }^{21} \gg$, en plus de proposer des discours féminins très crus sur la sexualité.

Dans le monde scientifique également, des auteures importantes issues des feminist cultural studies ou de la feminist television criticism ont formulé pareilles doléances à propos de Sex and the City et, plus encore, d'Ally McBeal. The Communication Review a publié en 2002 un ensemble d'articles très critiques à l'égard d'Ally McBeal. Bonnie Dow, Rachel Dubrofsky, Susan E. McKenna et Laurie Ouellette y interprètent les angoisses et mal-être affichés de l'héroïne comme un backlash antiféministe ${ }^{22}$. Pour L.S. Kim également, la série signifie que « Trop de choix, trop de liberté, trop de désir a mené à une quête sans fin et même à la dépression et au dysfonctionnement personnel $^{23}$. » Pour Dubrosfky, la nature d'Ally McBeal est idiosyncratique en ce qu'elle performe une « féminité névrosée » : capable de séduire rapidement et facilement, en accord avec l'image hégémonique de la femme glamour, elle trébuche néanmoins la seconde qui suit, ce qui « fétichise et esthétise la difficulté d'être une femme active ${ }^{24} \gg$, amenant les téléspectateurs à rire aux dépens d'une femme indépendante. Angela McRobbie souligne quant à elle le nouveau « régime de genre » auquel participent ces héroïnes, basé sur un « double enchevêtrement » entre des valeurs néo-conservatrices (axées sur la romance, la famille et le genre) et une liberté grandissante dans les rapports amoureux et sexuels ${ }^{25}$. Néanmoins, il n'existe pas, pour elle, de véritable tension : les valeurs traditionnelles se servent de pratiques faussement subversives, énoncées sur le registre de l'amusement, de la relaxation et du relâchement, pour se reproduire. 
D'autres féministes ont plutôt perçu des conflits genrés dans ces représentations humoristiques, notamment en termes de subjectivation et d'objectivation : pour Brenda Cooper ${ }^{26}$, la féminité des personnages d'Ally McBeal, aussi sexy et charmeuse soit-elle, ne se laisse pas soumettre à un male gaze classique. En retour, les masculinités représentées sont elles-mêmes comiques ou carnavalesques : l'un se donne de l'aplomb en chantonnant Barry White, l'autre se rassure en fumant de gros cigares et en payant des top-modèles vêtues de cuir pour qu'elles l'accompagnent dans chacun de ses déplacements. Rachel Moseley et Jacinda Read arguent de leur côté que la série n'est pas tant un « conflit entre la carrière et la vie personnelle qu'une lutte pour les faire tenir ensemble ${ }^{27} \gg$ : le fait de montrer les angoisses de la deuxième modernité n'est pas mécaniquement une apologie de la première modernité car il faut encore voir les modalités de ces angoisses, leurs principes, leurs contrecoups, leurs reformulations. En la matière, Michael Epstein a bien montré la perméabilité représentée dans la série entre les sphères publique et privée, qui inverse l'axiome féministe des années 1970 « le privé est politique » : dans Ally McBeal, « tout ce qui est professionnel est aussi personnel ${ }^{28} \gg$. Les collègues sont les amis, le cabinet d'avocat est une scène de jeux, les discussions de travail prennent des échos privés. S'ensuit une féminisation de la sphère professionnelle : l'environnement de Cage \& Fish déborde de rapports émotionnels, en ce que la majorité des cas juridiques relève de problèmes personnels ${ }^{29}$. Une approche plus culturelle de ces représentations a permis de dégager les controverses sociales qui les traversent. L'aspect plus récréationnel que relationnel des pratiques sexuelles est d'ailleurs confirmé dans l'analyse de contenu de la série menée par Gail Markle ${ }^{30}$ qui répertorie les pratiques des personnages. La conclusion plus qualitative d'une telle étude peut être trouvée chez Mandy Merck, qui affirme que si le fond des récits est hétérosexuel (les expériences homosexuelles sont rarissimes), leur forme est homosexuelle (puisqu'elle renvoie aux liens féminins) ${ }^{31}$. Pour Jane Gerhard, « l'angoisse postféministe - être libérée n'est pas ce que l'on nous a dit que cela serait est atténuée par la perspective queer de la série : l'amitié féminine devient la manière qu'ont les femmes de gérer ce qui est souvent représenté comme la solitude hétérosexuelle $e^{32} \gg$. À l'autre bout du spectre communicationnel,

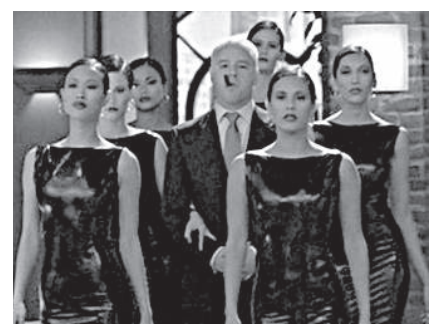


l'étude de réception de Susan McKenna entérine l'interprétation queer ${ }^{33}$ : les lesbiennes interrogées se réapproprient à la fois les corps des personnages qui ont souvent été critiqués comme dangereusement maigres (Ally) ou normés (Ling, Nelle, Georgia), ainsi que les liens érotiques entre les personnages féminins, dont on pouvait supposer qu'ils étaient simplement donnés au male gaze (par exemple, lorsque Ally apprend à Georgia comment boire de façon sexy un cappuccino, tandis que leurs collègues masculins les espionnent en se délectant du spectacle ${ }^{34}$ ).

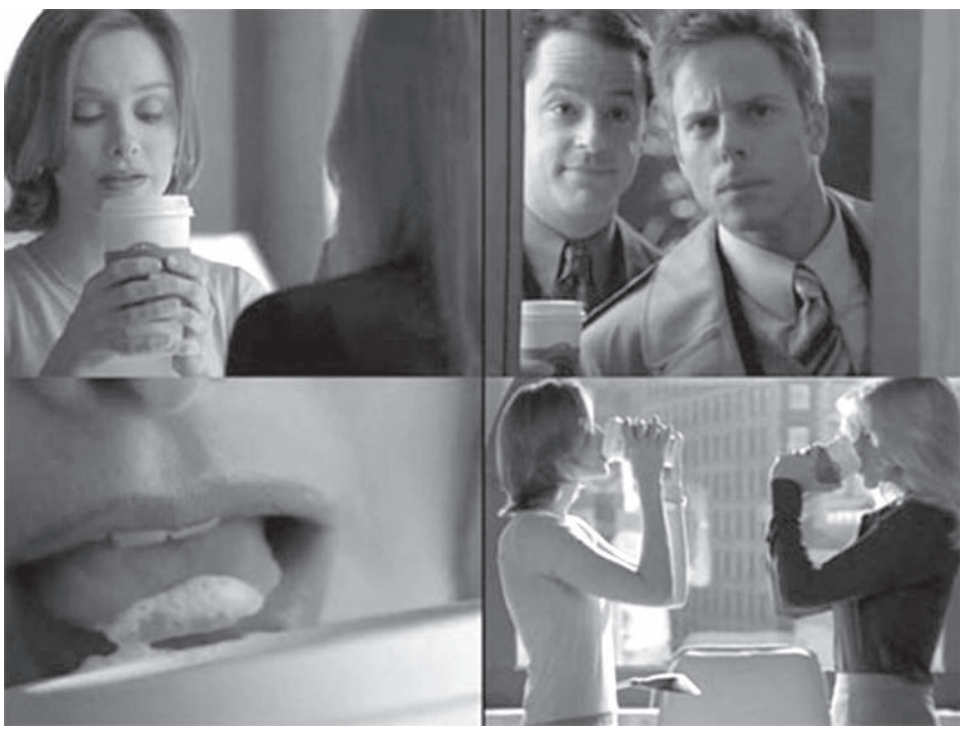




\section{Notes}

1 Lotz, Amanda D., Redesigning Women. Television after the Network Era, Urbana, University of Illinois Press, 2006.

2 Braidotti, Rosi, « Les sujets nomades féministes comme figure des multitudes $\gg$, Multitudes, 12, 2003.

3 Braidotti, Rosi, ibid.

4 Kaufmann, Jean-Claude, « La force structurante d'une illusion : l'individu », in Corcuff, Philippe, Le Bart, Christian, Singly (de), François, L'Individu aujourd' hui. Débats sociologiques et contrepoints philosophiques, Rennes, Presses Universitaires de Rennes, 2010.

5 Braidotti, Rosi, ibid.

6 Arthurs, Jane, « Sex and the City and Consumer Culture. Remediating Postfeminist Drama », Feminist Media Studies, 3, 1, 2003, p. 83-98.

7 Joss Whedon, commentaire DVD de Welcome to the Hellmouth.

8 Ally McBeal, saison 2, épisode 21.

9 Sex and the City, saison 3, épisode 10.

10 Gilmore Girls, saison 3, épisode 1.

11 Sex and the City, saison 1, épisode 7.

12 Hopkins, Patrick, « How Feminism Made a Man Out of Me: The Proper Subject of Feminism and the Problem of Men », in Digby, Tom, Bartky, Sandra, Men Doing Feminism, Londres, Routledge, 2008.

13 Digby, Tom, Bartky, Sandra, Men Doing Feminism, Londres, Routledge, 2008.

14 Harding, Sandra, «Can Men Be Subject of Feminism? », in Digby, Tom, Bartky, Sandra, ibid., p. 181.

15 Harding, Sandra, ibid., p. 183.

16 Beck, Ulrich, Beck-Gernsheim, Elizabeth, The Normal Chaos of Love, Cambridge, Polity Press, 1995, p. 13.

17 Ally McBeal, saison 1, épisode 6.

18 Sex and the City, saison 4, épisode 1.

19 Faludi, Susan, Backlash. La guerre froide contre les femmes, Paris, Des Femmes, 1993.

20 Bellafante, Ginia, « Feminist: It's All About Me! Want to know what today's chic young feminist thinkers care about? Their bodies! Themselves! », Time, 29 juin 1998.

21 Arthurs, Jane, « Sex and the City and Consumer Culture: Remediating Postfeminist Drama », Feminist Media Studies, 3, 1, 2003, p. 83-98. 
22 Bonnie, J. Dow, « Ally McBeal, Lifestyle Feminism, and the Politics of Personal Happiness », The Communication Review, 5, 4, 2002, p. 259-264 ; Dubrosky, Rachel, « Ally McBeal as Postfeminist Icon: The Aestheticizing and Fetishizing of the Independent Working Woman », The Communication Review, 5, 4, 2002, p. 264-284; McKenna, Susan E., « The Queer Insistance of Ally McBeal: Lesbian Chic, Postfeminism, and Lesbian Reception », The Communication Review, 5, 4, 2002, p. 285-314. Ouellette, Laurie, « Victims No More: Postfeminism, Television, and Ally McBeal », The Communication Review, 5, 4, 2002, p. 315-335.

23 Kim, L.S., « "Sex and the Single Girl” in Postfeminism: the F Word on Television », Television New Media, 2, 319, 2001, p. 319-334.

24 Dubrosky, Rachel, «Ally McBeal as Postfeminist Icon: The Aestheticizing and Fetishizing of the Independent Working Woman », The Communication Review, 5, 4, 2002, p. 264-284.

25 McRobbie, Angela, The Aftermath of Feminism. Gender, Culture and Social Change, Londres, Sage Publications, 2009.

26 Cooper, Brenda, « Unapologetic Women, “Comic Men” and Feminine Spectatorship in David E. Kelley's Ally McBeal », Critical Studies in Media Communication, 18, 4, 2001, p. 416-435.

27 Moseley, Rachel, Read, Jacinda, « “Having It Ally”: Popular Television (Post)Feminism », Feminist Media Studies, 2, 2002, p. 231-249.

28 Epstein, Michael, «Breaking the Celluloid Ceiling: Ally McBeal and the Women Attorneys Who Paved Their Way », Television Quaterly, 30, 1, 1999, p. 28-39.

29 Cooper, Brenda, ibid.

30 Markle, Gail, « Can Women Have Sex Like a Man? Sexual Scripts in Sex and the City », Sexuality \& Culture, 12, 1, 2008, p. 45-57.

31 Merck, Mandy, « Sexuality in the City », in Akass, Kim, McCabe, Janet, Reading Sex and The City, I.B. Tauris, 2003.

32 Gerhard, Jane, «Sex and the City. Carrie Bradshaw's Queer Postfeminism », Feminist Media Studies, 5, 1, 2005, p. 37-49.

33 McKenna, Susan E., « The Queer Insistence of Ally McBeal: Lesbian Chic, Postfeminism, and Lesbian Reception », The Communication Review, 5, 4, 2002, p. 285-314.

34 Ally McBeal, saison 1, épisode 8. 


\section{Fractures entre imaginaires, discours et pratiques}

\section{(Dé)constructions des amours}

À rebours de la définition romantique qui considère l'amour comme essentiel, éternel et exclusif, c'est-à-dire s'exprimant à un seul endroit et pour toujours, ses représentations sont désormais étendues et variées, concurrencées même par d'autres liens plus « faibles » comme les amitiés féminines. Contrairement aux séries précédentes où les héroïnes n'avaient qu'un amour central, peut-être deux, les « nouvelles "nouvelles femmes" » ont des relations qui évoluent en dents de scie. L'éclatement de la tradition fait qu'il n'est plus possible, comme le formule Ulrich Beck, « de dire de façon cohérente ce que [l'] amour signifie, ce qu' il devrait ou pourrait être : il varie en substance, exceptions, normes et moralités d'individu en individu, et de relation en relation ${ }^{1} \gg$.

De l'Amour aux amours, la perte de la tonalité romantique structure donc les redéfinitions. Car si l'amour est multiple, c'est un fondement du romantisme qui s'effrite. Les dragons qu'affronte le Prince charmant sont désormais de son espèce et les héroïnes, ne voulant plus être subsumées dans l'ensemble conjugal, doivent choisir entre ces différentes propositions amoureuses. Carrie Bradshaw en est un bon exemple, qui s'affranchit même des petits amis, accumulant les relations : « ce n'est pas mon copain, c'est juste quelqu'un que j'essaie ${ }^{2} \gg$, dit-elle, qu'elle essaie jusqu'à décider s'il lui convient ou non, si elle-même se convient ou non avec lui. La précarité des amours se confond avec la précarité des identités : s'il est si difficile de hiérarchiser ces diversités affectives, c'est que ce choix n'est pas seulement celui d'un homme ou d'un autre, c'est aussi celui d'une identité future. 
Cela explique la présence d'une rupture très franche entre les discours et les actes : si les trentenaires disent chercher l'homme idéal, leurs amours relèvent davantage de la sexualité plastique et de la relation pure.

Les imaginaires, pratiques et discours ne sont pas cohérents. Les discours sont tiraillés entre imaginaires romantiques et pratiques de relations plus « pures » : les héroïnes disent vouloir un amour illogique, au-delà des intérêts individuels, qui consume, mais en parallèle, elles se plaignent des résistances sexistes qui y sont à l'œuvre. En retour, les pratiques sont en décalage avec les imaginaires et les discours : à travers les saisons, les héroïnes de Sex and the City et d'Ally McBeal sont essentiellement engagées dans des formes embryonnaires de relation pure et, lorsque le Prince charmant se présente à elles, il ne les convainc pas forcément. Carrie est troublée de ce que sa romance avec Aidan ne la comble pas... précisément parce qu'elle est parfaite :

CARRIE : - Ce qui ne va pas, c'est que, pour la première fois de ma vie, je suis dans une relation où absolument rien ne va pas. On navigue en douceur. Rien d'autre qu'une mer calme, de l'horizon bleu partout où je regarde. [...] C'est bizarre. Je suis habituée à chasser. Là, c'est sans effort. Ça me fait flipper ! ${ }^{3}$

Imaginaires, pratiques et discours ne sont pas cohérents car les modèles mis à disposition ne le sont pas eux-mêmes. Comme l'a bien montré Joanna di Mattia, Mister Big et Aidan incarnent chacun à leur manière l'idéologie romantique. Le premier personnifie un romantisme passionnel tandis que le second propose un romantisme stabilisant. Or, qu'il s'agisse de la virilité classique de Mr Big ou de la masculinité postféministe d'Aidan (« mélange du héros phallique avec le nouvel homme sensible ${ }^{4}$ )), chacun des personnages est perçu comme compatible avec le romantisme car Carrie projette sur eux ses fantasmes : ses attentes romantiques précèdent les partenaires qu'elle rencontre. Lorsque Carrie s'inquiète que tout soit parfait, elle regrette en fait qu'il n'y ait pas plus de ferveur affective, ce qui comprend des moments de dispute codés passionnels. De son côté, Ally rencontre à plusieurs reprises des hommes potentiellement idéaux auxquels elle trouve invariablement un défaut rédhibitoire : l'héroïne est guidée par une utopie qui piège les pratiques. 


\section{Labeur individuel, idéal de l'aisance romantique}

Les récits sont de plus en plus ceux de la construction des amours à partir d'interactions et de dosages inédits entre les modèles disponibles et des identités malléables. Les héroïnes ne se contentent pas de résoudre les problèmes que pose leur mariage comme Maude et Roseanne mais élaborent démocratiquement la structure conjugale. Les temporalités sont inversées : le point de départ est l'attraction physique et psychologique qui, peu à peu, mène à la relation amoureuse, laquelle demande du temps, de l'investissement, des compétences émotionnelles et des valeurs égalitaires. La construction de la relation amoureuse s'effectue par paliers. Les premiers rendez-vous, traditionnelles dates étasuniennes, deviennent le lieu d'un jeu amoureux fait de performances de genre classiques, que les femmes décrivent ensuite comme des jeux théâtraux aux allures goffmaniennes : «Charlotte me dira plus tard qu'elle pensait avoir joué la soirée sans aucun faux pas $^{5} \gg$, raconte Carrie Bradshaw qui de son côté regrette d'avoir porté pour son premier rendez-vous avec Mr Big une robe dénudée qui les a encouragés à faire l'amour sans attendre. Comme l'a montré Beth Bailey, les codes communicationnels qui structurent les premiers rendez-vous amoureux sont hérités de la traditionnelle cour amoureuse américaine mais ils sont en même temps perturbés par les nouveaux rapports de genre : les « dissonances » que produisent ces derniers s'expriment « dans des luttes pour gagner ou regagner du contrôle dans le système de cour $^{6} \gg$. Carrie craint en effet que le contrôle sexuel qu'elle exerce lorsqu'elle se montre presque nue n'aboutisse paradoxalement à une domination masculine: ayant épuisé tout son capital sexuel dès le premier soir, l'héroïne a peur de n'être plus émotionnellement crédible auprès de Mr Big. La part grandissante de construction dans les rapports amoureux a ses symptômes que la littérature scientifique a bien identifiés: la labilité apporte son lot de névroses et de « fatigues d'être soi ${ }^{7} \gg$. Lorsque commence sa relation avec Mr Big, Carrie est épuisée de devoir continuer de performer une féminité parfaite: «Quand je suis avec lui, je ne me ressemble pas, je suis comme une "Carrie contenue", je mets des petits costumes, tu sais, "Carrie sexy" et "Carrie décontractée”. Parfois, je me surprends à poser ! C'est crevant ! ${ }^{8}$ » Les femmes sont soumises à une représentation constante d'elles-mêmes 
qui, parce qu'elle est censée agir comme une preuve de leur affection, rend prioritaire la performance de genre sur la démocratie intime.

Dans le même temps pourtant, s'affaiblit l'idéal de la communication romantique, supposément immédiate et aisée grâce à la fusion et à l'intuition. Certes, des formes romantiques persistent comme des échanges de regards (entre Ally et Billy ou Carrie et Big), des chamailleries complices entre Lorelai et Christopher (Gilmore Girls), ou des élans lyriques (Buffy, Charmed). Mais, en parallèle, apparaissent de nombreuses occurrences d'un labeur relationnel et d'une démystification de l'intercompréhension immédiate. La romantique Ally McBeal est déconcertée lorsque Larry refuse de participer à un échange fusionnel et qu'il défend des découvertes progressives basées sur le non-verbal ${ }^{9}$. Le travail relationnel croît en interaction avec l'idéal de la communication romantique qu'il contredit souvent, mais les héroïnes, prises aux pièges d'un système de genre encore très binaire, sont laissées face à une injonction contradictoire : elles doivent, tout en restant éminemment séduisantes, faire l'effort de s'ouvrir psychologiquement à l'autre. La solution qu'elles trouvent est typique de leur statut de femme active. Rêvant toujours du Prince charmant, les femmes ne sont plus des princesses passives pariant sur les faveurs du destin : pour trouver l'élu de leur cœur, elles multiplient les relations pures en travaillant à ce que l'une d'elles se transforme en conte de fées, tant et si bien que le hasard semble ne plus avoir sa place dans les récits. L'amour perd de sa destinée et s'imprègne de la contingence moderne, il est désormais une entreprise personnelle au même titre qu'une carrière : pour Ally McBeal, « Nous consacrons douze heures par jour à notre vie professionnelle tout en déclarant que notre vie personnelle est plus importante mais nous n'y travaillons pas. Je vais appliquer un plan de carrière dans ma vie privée. Au lieu de me croiser les bras en espérant que ça arrive, je vais m'arranger pour que ça arrive $!^{10} \gg$ Cupidon est au chômage. Un glissement opère, qui espère aisément déplacer les compétences professionnelles vers la sphère amoureuse, ne voyant pas de raison à ce que la détermination personnelle qui a permis l'accomplissement professionnel ne puisse faire aussi ses preuves dans le domaine amoureux. Les amours deviennent des objets de travail. Le tournant est flagrant : tandis que les femmes actives et les « féministes au foyer » critiquaient les sacrifices que requiert une relation, les héroïnes des années 1990 se concentrent sur le labeur émotionnel et 
communicationnel en ramenant dans la sphère privée les compétences développées dans la sphère publique. Une fois la relation sérieusement enclenchée, l'amour ne doit plus demander des sacrifices mais du temps, des encouragements et une constante considération. Si les comportements amoureux restent évidemment structurés par les rapports de genre, les logiques individualistes interviennent de plus en plus. Néanmoins, les héroïnes oublient que le caractère souvent hasardeux de la rencontre amoureuse empêche de maîtriser totalement ses manifestations. Cupidon est au chômage mais il n'est pas mort.

\section{Imaginaires idéalisés sur un modèle romantique concrètement affaibli}

Indice de sa résistance, les modalités représentationnelles du romantisme le rattachent à une croyance quasi religieuse en l'amour. Dans The Normal Chaos of Love, Ulrich Beck et Elisabeth Beck-Gernsheim avaient déjà proposé une telle définition, envisageant l'amour comme une « religion après la religion, l'ultime croyance après la mort de toutes les fois ${ }^{11} \gg$. Selon eux, l'amour promet aujourd'hui de résoudre le problème contemporain de la solitude tout en offrant à l'individu une stabilité liée à la personne : « Si ce n'est plus Dieu ou les prêtres, ou la classe sociale ou les voisins, alors au moins, il y a toujours Toi $^{12}$. » Une telle approche trouve un écho dans les représentations où la récompense du cheminement individualiste prend la forme de l'individu destiné. Élu du cœur, moitié, âme sœur ou Prince charmant sont le Saint Graal de l'amour qui conclut de nombreuses séries sur un happy end romantique (Sex and the City, Charmed, Alias, Gilmore Girls, voire également le romantisme parental d'Ally $M c B e a l)$. Face à un processus d'individualisation anxiogène, entre rejet du holisme et crainte de la solitude, le romantisme promet un séduisant compromis : il concilie sécurité et indépendance et investit la place laissée vacante par les structures familiales rigides. L'identité individuelle qui n'est plus validée par les structures dont elle s'est émancipée trouve reconnaissance auprès de l'unique pour toujours - la contrepartie étant une « disparition de soi dans une totalité ${ }^{13}$ » romantique qui grève plus particulièrement le féminin du fait de son assignation au privé. 
Les références religieuses sont nombreuses et explicites. Les hérö̈nes convoquent un champ lexical religieux, évoquant la «croyance », la « foi », les « signes », et s'entendent dire en retour qu'elles s'illusionnent dans des idéaux. Ally McBeal voit dans la croyance en l'amour le courage pour supporter sa solitude : « J'ai besoin de croire que ça marche - l'amour, le couple, le partenariat, l'idée que quand des gens se mettent ensemble, ils restent ensemble. J'ai besoin d'emmener ça avec moi le soir dans mon lit, même si je me couche seule ${ }^{14}$. » Dans Alias, Sydney est furieuse que deux ans seulement après sa disparition, Vaughn, déjà remarié, n'ait pas cru dans leur relation : «Tu veux savoir comment je vais ?Je suis dévastée, Vaughn, je suis anéantie! Pas parce que je t'ai perdu... mais parce que si ça avait été moi, j'aurais attendu. J'aurais découvert la vérité. Je ne t'aurais pas abandonné. Et maintenant, je réalise quel incroyable gâchis cela aurait été ${ }^{15}$. » De leur côté, les héroïnes de Sex and the City passent un épisode entier à essayer de savoir si « les relations amoureuses sont la religion des années $1990^{16} \gg$. Se trouvant dans une impasse car Mr Big refuse de la présenter à sa mère, Carrie est désespérée : « J'ai besoin d'un signe, tu me dis d'avoir la foi mais je la perds alors j'ai besoin d'un signe. [...] Dis-moi juste que je suis la bonne, tu n'as pas à le dire à ta mère ni au monde entier, juste... juste moi $^{17}$. » Carrie produit ici un compromis paradoxal en demandant à Big de lui confirmer, à elle seule, qu'elle est « la bonne ». Elle accommode ainsi son idéal romantique aux impératifs individualistes : la confirmation romantique se fait dans l'échange interpersonnel sans qu'il soit besoin de prendre pour témoin les structures classiques. L'effervescence romantique reste, elle, non négociable : Carrie n'accepte pas que Mr Big lui demande d'avoir foi en l'avenir car cela entre en collision avec ses idéaux d'un amour romantique par définition immédiat et entier. Cela pose la question de ce qui, des sentiments ou de la relation, apparaît en premier : si Carrie admet que la relation s'élabore par compromis, elle refuse que les sentiments apparaissent au même moment ou, pire, à postériori.

D’un autre côté, le monde désenchanté d'Ally McBeal produit ses propres paradoxes : plus l'idéal romantique est obsolète, plus il est renforcé chez l'hérö̈ne. Les cas judiciaires qu'Ally traite la confrontent sans cesse aux échecs du modèle de la famille nucléaire. Elle est le témoin, et ironiquement l'avocate !, d'une pluralisation des modèles amoureux et familiaux : ménages à trois, familles homoparentales, transexuel.le.s se 
succèdent à la barre pour demander à une législation désuète la reconnaissance du droit de vivre comme ils l'entendent. En parallèle, les cas hétérosexuels sont bien souvent ceux d'un déchirement ou d'une résignation. Dans cet effondrement du romantisme, Ally incarne un sursaut dont la forme illusionnée est un mécanisme de défense contre le monde incertain qu'elle côtoie au quotidien. Les racines de son idéalisation de l'amour sont précisément consolidées par cette précarité qu'elle a expérimentée très tôt. Une dispute entre l'héroïne et sa mère renseigne en effet le téléspectateur sur les précoces rêvasseries d'Ally:

JEANNIE, mère d'Ally : - Ally, tu sais quoi, reste donc dans tes rêvasseries. [...]

Ally : - Je ne vis pas dans des rêvasseries, maman ! C'est juste que je m'y réfugie parfois !

JEANNIE : - Non, le problème, c'est que tu les prends pour la réalité. Eh bien, pas moi.

Ally : - Non. Une rêvasserie, c'est quand je vais dans ma chambre, que je ferme les yeux et que je vois une licorne. La réalité, c'est quand, à trois ans, j'entre dans la chambre de mes parents et que je te vois au lit avec un autre homme. Je sais faire la différence ${ }^{18}$.

Le romantisme, qui prend historiquement racine dans la construction d'une individualité contre les structures sociales, est dans Ally McBeal le symptôme d'un traumatisme enfantin. Ally s'illusionne de plus belle car elle a été désillusionnée trop tôt : l'héroïne rendue hyper-sensible trouve dans la fluidité romantique la promesse d'ignorer la réalité crue qui s'est imposée à elle.

En ce sens, de la même façon que s' influencent précarité identitaire et précarité a moureuse, l'idéalisation de l'amour renvoie à un idéal du moi, désenchevêtré des contingences pragmatiques de la deuxième modernité. Un tel jeu entre la représentation idéalisée de l'amour et son évocation explique sa persistance dans les médiacultures, malgré son incapacité à se réaliser concrètement. Le romantisme est ainsi conjointement « abymé » et abîmé : il est «abymé », mis en abyme, car ce modèle se retrouve confiné aux rêves des hérö̈nes, aux imaginaires des imaginaires médiatiques; il est aussi abîmé, endommagé, car ses seuls accès au premier 
niveau de représentation s'effectuent pour signifier que les hérö̈nes ne souhaitent pas vraiment l'actualiser. Les fins des séries sont un bon indice des allées et venues de l'optimisme et de la méfiance quant aux promesses de l'amour. Après avoir majoritairement vécu des relations incertaines, avortées parce qu'elles contrariaient l'épanouissement individuel, les héroïnes des années 1990 trouvent le bonheur, et ce de manière souvent exagérée, comme pour récompenser leurs efforts : Sydney (Alias) part vivre sur une plage déserte avec son mari et ses enfants; les derniers plans de Sex and the City montrent chacune des héroïnes en couple, alors qu'elles sont celles qui ont le plus pratiqué la relation pure ; Max et Logan (Dark Angel) sous-entendent qu'ils surmonteront la malédiction qui les empêche de se toucher en joignant leurs mains gantées; Dharma et Greg ainsi que Lorelai et Luke continuent de vivre amoureux ; Charmed montre les héroïnes devenues vieilles entourées de leur mari et de leurs enfants, rejouant (littéralement !) le très classique «Ils vécurent heureux et eurent beaucoup d'enfants. » Seul l'amour de Buffy et Angel, qui est pourtant le plus romantique des séries analysées ici, reste inachevé en accord avec les tonalités pessimistes de la série sur l'a mour et la sexualité. Ces fins romantiques tranchent radicalement avec les représentations produites tout au long des saisons. Une chronologie est commune aux séries : dans un premier temps, les héroïnes ont des relations amoureuses qui relèvent de la relation pure ou qui portent en elles des fragments de la relation pure comme la sexualité épanouissante (Sex and the City), la démocratie relationnelle (Dharma \& Greg) ou l'attention à l'égalité (Gilmore Girls). Ces amours n'existent toutefois pas pour elles-mêmes mais apparaissent comme des moyens pour les héroïnes de déterminer ce qu'elles veulent et ce qu'elles ne veulent pas, un ensemble d'expériences au cours desquelles les femmes, qui ont tiré les leçons de la réflexivité et considèrent qu'il faut être bien avec soi-même avant d'être bien avec quelqu'un, se découvrent. Dans un deuxième temps, qui n'est toutefois pas exploré puisqu'il s'agit de la fin des séries, les femmes accomplissent le rêve romantique, faisant de leurs relations passées des préludes à cet accomplissement traditionnel. La relation pure aura été un modèle adéquat aux premiers moments d'une relation amoureuse dans laquelle on ne peut plus immédiatement s'engager. 
La fracture entre imaginaires et pratiques se résout donc dans une instrumentalisation de la relation pure, en tant que matrice d'expériences personnelles au service de l'accomplissement romantique. La labilité amoureuse apparaît comme la possibilité de « tester » des relations et des hommes jusqu'à trouver celui que les héroïnes n'appellent plus vraiment le Prince charmant, mais le Bon, « the rightguy », « Mister Right ». Ce glissement terminologique témoigne de l'affaiblissement de la croyance en l'homme parfait, au profit d'une figure individualisée de l'Élu amoureux. « Monsieur » est « le Bon », non pas intrinsèquement, mais par rapport aux attentes et aux particularités de celle qui le cherche : l'amour perd de sa substance totalisante pour embrasser les trajectoires individuelles. Jusqu'en 2004, date d'arrivée d'héroïnes quadragénaires qui inverseront cette temporalité « relation pure puis romantisme », la relation pure n'est donc pas une fin mais un moyen pour tester les potentiels princes charmants. Elle est curieusement, ce que Giddens n'envisage pas, une forme embryonnaire de l'amour romantique auquel elle s'oppose.

\section{Dharma \& Greg ou la rare réussite démocratique}

En termes de démocratie relationnelle, l'unique franche réussite de la télévision étatsunienne est Dharma \& Greg. Cela n'est pas sans lien avec l'évolution des modèles amoureux qui y est représentée : contrairement à Sex and the City ou Ally McBeal où les relations pures précèdent le romantisme, le pilote de Dharma \& Greg propose une vision immédiatement romantique de leur couple. Tout d'abord, la première scène relève de la prédestination : elle les montre, enfants, se croisant dans le métro, et enchaîne avec leur rencontre, quelques décennies plus tard, au même endroit. Puis, leur relation se fait contre leurs origines sociales : Dharma, professeure de yoga, est née dans une famille hippie, tandis que Greg, avocat, a grandi dans un foyer bourgeois. La base de leur relation est donc le thème amoureux de l'elope, de la fuite pour se marier contre les structures familiales. Il s'agit là d'une rare occurrence d'un écart de classe dans les représentations des années 1990. Même s'il n'est pas sans causer problèmes, c'est précisément l'apport mutuel d'une perspective différente sur le monde qui enrichit la relation : dans «Chacun son dû ${ }^{19}$ », Dharma remercie Greg de l'avoir stabilisée et Greg 
remercie Dharma de l'avoir rendu plus serein. Néanmoins, la pérennité de leur relation ne repose pas sur ce romantisme à l'origine de leur relation mais sur des mécanismes démocratiques, comme en témoigne l'usage répété et systématique de confiance, d'encouragement et d'honnêteté pour résoudre leurs problèmes. De très nombreuses répliques parsèment la série, insistant sur le respect du partenaire, comme par exemple lorsque Greg avoue : «Je ne devrais pas te freiner dans ce que tu veux faire simplement parce que je ne le comprends pas, je devrais accepter que c'est important pour toi, peu importe ce dont il s'agit ${ }^{20}$. » Ces difficultés se résolvent presque invariablement lorsque l'un des partenaires réalise que le problème n'est pas l'autre et ses envies, mais ce que lui-même redoute. Dans Dharma \& Greg, les peurs individuelles freinent l'intercompréhension car elles freinent en fait l'expression individualiste d'un « je » hors du « nous »: Dharma, qui veut partir seule faire une retraite spirituelle, explique à Greg « Je ne suis pas inquiète à propos de nous, d'accord ? Je suis inquiète à propos de moi. Le moi qui n'est pas nous. Non pas qu'il y ait un moi qui ne veuille pas d'un nous. C'est juste que ce sera un meilleur "nous" si je sais qui "moi" $e^{21}{ }^{21}$. $\gg$ Comme toujours, Greg accepte finalement. Le romantisme est ainsi surplombé par la relation pure jusqu'à disqualifier l'idéal si présent dans les représentations contemporaines d'un «Mr Right». Quand Dharma cherche un potentiel partenaire pour l'ex-petite amie de Greg, elle rencontre un homme hippie en apparence parfait pour elle : leur communication est facile, immédiate et intuitive car, provenant du même milieu, ils partagent les codes communs de l'idéologie new age. Lorsque Greg les interrompt, demandant à Dharma si elle a trouvé « Mr Right » (sous-entendu pour son ex-petite amie), elle lui répond «Ouais, mais je rentre avec l'homme que j'aime ${ }^{22}$. » Les représentations hiérarchisent ici l'amour actuel et travaillé, sous-entendant que l'intercompréhension immédiate est due à un déterminisme sociologique qui ne promet pas forcément une réussite émotionnelle. Écouter son partenaire et ne pas lui infliger ses propres peurs apparaissent en retour comme les deux clés de voûte de la réussite démocratique du couple. Elles peuvent être développées grâce à l'idéologie hippie de Dharma qui crée un amour dont les valeurs dominantes sont la compassion et la bienveillance, lesquelles engendrent et sont engendrées par la confiance. De fait, lorsque cette confiance est trahie, c'est toute la relation qui chancelle. 
Après que Greg a dormi chez son ex-petite amie, Dharma est furieuse pour une raison inattendue :

Dharma : - Tu as dormi chez ton ex-petite amie !

GREG : - Il ne s'est rien passé.

DHARMA : - Je sais bien parce que je te fais confiance. Mais tu ne m'as pas laissé une chance de te faire confiance, tu m'as juste menti ${ }^{23}$.

Dans la série, les problèmes sont invariablement résolus par des excuses ou par une explication, c'est-à-dire par un cheminement personnel. Ils relèvent ainsi bien plus d'une réflexivité individualiste visant à ne pas écraser l'autre à cause de ses propres limites de compréhension. Pour pérenniser l'ouverture à l'autre et le pardon, les partenaires se soumettent à des exercices new age, par exemple lorsqu' ils enferment des secrets jusqu'ici inavoués dans des bulles

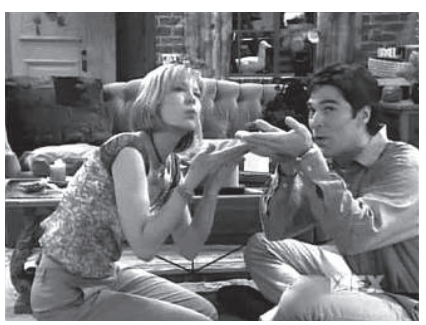
imaginaires qu'ils soufflent au $\operatorname{loin}^{24}$. Ce n'est pas un hasard si le succès du couple est bien souvent dû aux techniques hippies de Dharma et à la volonté de Greg de s'y convertir. Les différences ne sont pourtant pas entendues comme un déséquilibre mais comme une richesse multiculturelle. L'invocation des valeurs orientales permet un décentrement de l'amour occidental (structuré dans et par la famille nucléaire) mais elle est aussi permise par l'intérêt grandissant donné au cours de la deuxième modernité aux nœuds psychologiques.

L'idéologie new age de Dharma est très précieuse pour la relation pure parce qu'elle considère que tout a un sens, un but, une utilité. La moindre dispute apparaît alors comme l'opportunité individuelle et conjugale de se comprendre, de s'expliquer et surtout de s'améliorer. Après une dispute, que l'on peut considérer comme la plus violente de la série car toute communication est rompue pendant trois jours, Dharma peine à faire comprendre à Greg que la pire chose n'est pas de se disputer mais de considérer qu'il y a un vainqueur : «On peut prendre ça sous l'angle de qui a fait quoi à qui et additionner les points mais on va finir avec un énorme tableau des scores au lieu d'un mariage ${ }^{25}$. » Enfin, dernier élément, la sexualité des personnages 
est cruciale : pratiquée tous les jours, elle foisonne d'innovations qui la décentrent ainsi de la sexualité hétéronormée. Orientée vers le plaisir, elle est un moyen de communication et un signe de la bonne santé du couple : lorsqu'épuisés, Dharma et Greg s'endorment durant les préliminaires, ils s'inquiètent de la vigueur de leur couple et décident de pimenter leur vie sexuelle en faisant l'amour dans des endroits incongrus ${ }^{26}$. Leur recours au Kâma-Sûtra sous-entend par ailleurs que Dharma et Greg ne réduisent pas la sexualité à la pénétration et qu'ils développent un ars erotica coïncidant avec la sexualité plastique.

La réussite du couple n'a donc pas trait au coup de foudre mais au travail identitaire et relationnel produit tout autant dans le couple qu'en dehors. L'idéologie new age qui amène à vouloir se comprendre soi-même se superpose sans difficulté à l'individualisme réflexif : il s'agit non seulement d'être bien avec soi-même, mais aussi et surtout de réussir à atténuer les répercussions négatives qu'ont nos angoisses sur autrui. La psychologisation forte de la relation amène une grande nouveauté : à l'heure où les autres héroïnes sont peu conciliantes car elles se sentent individuellement menacées par le compromis conjugal, Dharma tout autant que Greg admettent leurs torts, s'excusent, acceptent leurs différences, revoient leur perspective. Les changements auxquels ils consentent ne se font pas en fonction de l'autre ou en fonction du couple mais ils se font avec l'autre, simultanément pour soi, pour autrui et pour le couple. Le changement que produit forcément l'entrée dans une structure de couple délaisse l'adaptation à l'autre, qui historiquement a été celle du féminin au masculin, au profit d'une transformation mutuelle, entendue comme une opportunité individuelle de se découvrir et de grandir. 


\section{Notes}

1 Beck, Ulrich, Beck-Gernsheim, Elizabeth, The Normal Chaos of Love, Cambridge, Polity Press, 1995, p. 5.

2 Sex and the City, saison 1, épisode 3.

3 Sex and the City, saison 3, épisode 7.

4 Di Mattia, Joanna, ibid.

5 Sex and the City, saison 1, épisode 1.

6 Bailey, Beth, From Front Porch to Back Seat. Courtship in TwentiethCentury America, Baltimore, Johns Hopkins University Press, 1989.

7 Ehrenberg, Alain, La Fatigue d'être soi. Dépression et société, Paris, Odile Jacob, 2000.

8 Sex and the City, saison 1, épisode 11.

9 Ally McBeal, saison 4, épisode 7.

10 Ally McBeal, saison 2, épisode 21.

11 Beck, Ulrich, Beck-Gernsheim, Elizabeth, ibid., p. 12.

12 Beck, Ulrich, Beck-Gernsheim, Elizabeth, ibid., p. 33.

13 Kaufmann, Jean-Claude, L'Étrange histoire de l'amour heureux, Paris, Fayard, 2010.

14 Ally McBeal, saison 1, épisode 2.

15 Alias, saison 3, épisode 1.

16 Sex and the City, saison 1, épisode 12.

17 Sex and the City, saison 1, épisode 12.

18 Ally McBeal, saison 3, épisode 5.

19 Dharma \& Greg, saison 4, épisode 10.

20 Dharma \& Greg, saison 5, épisode 8.

21 Dharma \& Greg, saison 2, épisode 9.

22 Dharma \& Greg, saison 1, épisode 5.

23 Dharma \& Greg, saison 2, épisode 12.

24 Dharma \& Greg, saison 3, épisode 20.

25 Dharma \& Greg, saison 4, épisode 24.

26 Dharma \& Greg, saison 1, épisode 22. 



\section{Nouvelles subjectivités charnelles et communications des désirs-plaisirs}

\section{Un nouveau Levier identitaire}

Détachée depuis un demi-siècle du mariage et de la reproduction, la sexualité est reconnue plurielle et réflexive sous l'impulsion des mouvements d'émancipation en même temps que ses normes médico-sociales, comportementales ou genrées continuent de s'actualiser. Devenue « plastique » pour Anthony Giddens ou « autonomisée » pour Michel Bozon ${ }^{1}$, c'est-àdire détachée de sa fonction reproductive, la sexualité se trouve désormais chargée des micropolitiques identitaires de l'individu. Elle devient, non plus un moyen procréateur, ni une légitimation du couple, mais un levier identitaire au service de la conjugalité.

L'importante pudeur des héroïnes de séries télévisées jusqu'aux années 1990 rend d'autant plus importante la libération sexuelle des « nouvelles “nouvelles femmes" ». À partir des années 1990, les héroïnes trentenaires interrogent les conséquences qu’a eues le féminisme sur les pratiques amoureuses et sexuelles. En parallèle, les pratiques deviennent libérées et extatiques : les héroïnes n'ont désormais plus besoin de sentiments pour coucher et la sexualité se fait épisodique, au gré des envies, enrichie même de techniques de soi qui encouragent la subjectivation comme les vibromasseurs et autres jouets sexuels. Ainsi autonomisées, les pratiques sexuelles permettent d'évoquer l'émancipation féminine et ses conséquences (dénaturalisation ou réification du genre) ainsi que les 
rapports de pouvoir entre hommes et femmes. Ce glissement fait largement disparaître le moralisme (les pratiques marginales sont peu dénoncées) au profit de morales individualistes sous-tendues par les enjeux féministes de la réappropriation des corps, de l'acceptation des désirs et du dépassement des doubles standards. Ally McBeal en est un bon exemple puisqu'elle insiste tantôt sur son droit à porter des jupes aussi courtes qu'elle le veut ${ }^{2}$, tantôt à laisser s'exprimer ses pulsions sexuelles ${ }^{3}$. L'innovation majeure, qui a certainement été la plus traitée, est la capacité de ces personnages féminins à adopter des comportements jusqu'ici réservés au masculin : les femmes s'assument sexuellement et multiplient les conquêtes. La raison en est que la sexualité, qui n'est plus contenue exclusivement à la fusion romantique ni au simple partage émotionnel, s'autonomise en s'orientant davantage vers le plaisir. En passant du couple à l'individu, les pratiques sexuelles se chargent de nouvelles significations qui ne renvoient plus seulement au bien-être conjugal mais qui ne se limitent pas pour autant au bien-être individuel. La sexualité des femmes relève désormais de l'épanouissement personnel et revendique le droit de disposer de son corps, se télescopant avec la question des identités féminines dans une société plus sensible aux égalités de genre. S' interrogeant sur le nombre de partenaires qu' il est possible d'avoir avant que cela ne devienne trop, Carrie se demande : «Sommes-nous des handicapées romantiques ou tout simplement des salopes $?^{4} \gg$ Cette thématique de l'épisode va au-delà du double standard qu'elle critique par ailleurs (« Les hommes qui ont eu beaucoup de partenaires sexuelles ne sont pas qualifiés de salopes, on dit qu'ils embrassent bien, on dit même de certains qu'ils sont romantiques. ») : elle interroge surtout la possibilité pour les femmes d'un juste milieu entre une sexualité jugée romantique et une autre jugée débauchée. In fine, c'est de la pluralisation des identités sexuelles et de leurs rejaillissements sur les identités sociales dont il est question.

Dans une thématique supposément aussi biologique que la sexualité, les problèmes ne se posent donc pas quant à l'usage des corps (les héroïnes n'ont aucun remords vis-à-vis de leurs pratiques) mais quant aux symboliques qui s'y rattachent. L'ambiguïté des représentations sexuelles de Sex and the City repose précisément dans cette scission qui n'opère pas, comme pour les modèles amoureux, entre ce que les héroïnes disent vouloir (le Prince charmant) et ce qu'elles font (des relations pures), mais entre ce qu'elles veulent faire (et qui est plutôt en accord avec ce qu'elles font) et 
l'image qu'elles veulent renvoyer d'elles-mêmes. Lorsque son petit ami veut pratiquer la sodomie, Charlotte exemplifie parfaitement cette situation :

$\ll$ Charlotte :-Je ne peux pas, Brian. Je voudrais bien, mais je ne peux pas. Enfin, en fait non, ce n'est pas vrai, je ne veux pas. Ou peut-être que si. Je ne sais pas ce que je veux. Mais j'ai peur que, si je ne le fais pas, tu me plaques. Et que, si je le fais, je serai la fille-par-derrière. Et je ne veux pas être la fille-par-derrière, parce que... les hommes n'épousent pas la fille-par-derrière! Qui a déjà entendu parler de Madame Par-Derrière ? Non, non, non, je ne peux pas.

Or, si Charlotte refuse, ce n'est pas parce que la pratique lui déplait (elle ne sait pas si elle veut le faire ou non), mais parce qu'elle en craint les conséquences sur son identité conjugale. Les hérö̈nes se brûlent les ailes lorsqu'elles découvrent que la réappropriation individuelle se heurte à la persistance des normes sociales.

Témoin de la rationalisation de l'amour, la métaphore économique se diffuse par ailleurs en profondeur dans les années 1990. La « compétition » entre femmes, les « stocks » de célibataires, l'« attractivité » physique, la « consommation » amoureuse et sexuelle sont autant de thèmes qui laissent entrevoir une économie de l'amour, où se jouent les valeurs des individus et leur capacité à résister à la concurrence. Le premier problème est celui du stock. Les femmes sont désemparées face à la pénurie d'hommes célibataires : dès le pilote de Sex and the City, Carrie souligne ce décalage. Vantant les qualités des femmes modernes, elle envisage le marché amoureux de New York comme une aporie : « Pourquoi y a-t-il tant de femmes fabuleuses et célibataires, mais aucun homme fabuleux et célibataire $?^{6} \gg$ Cela fait émerger, c'est un deuxième problème, une rivalité. Les femmes sont en compétition pour « attraper » le masculin lorsqu'il est libre : Ally, qui est toujours sous le charme de son amour de jeunesse Billy, est en concurrence avec l'épouse de ce dernier, Georgia, qui se sent en retour menacée dans son couple. Dans Sex and the City, cette rivalité est rendue plus difficile encore par la capacité qu'ont les hommes à sortir avec plusieurs femmes en même temps. Alors qu'elle considère être en couple « exclusif » avec $\mathrm{Mr}$ Big, Carrie réalise qu'il continue de voir d'autres prétendantes. 
Elle s'interroge : «Tandis que pour moi, voir un autre homme serait comme essayer de caser une tenue supplémentaire dans une valise déjà trop remplie, Big sortait joyeusement avec d'autres femmes comme si c'était la chose la plus naturelle au monde. [...] Dans une ville comme New York, avec ses possibilités infinies, la monogamie était-elle trop demander ? $^{7}{ }$

Hommes et femmes ne sont pas égaux face aux prérogatives du marché amoureux car l'hétérogénéité des profils féminins rend la concurrence plus dure. De plus, l'ethos féminin, supposément plus scrupuleux, les empêcherait de profiter pleinement du marché (et de faire jouer la concurrence, comme le font les hommes), parce que, déjà émancipées dans la sphère publique, elles risqueraient de faire fuir le masculin si elles l'étaient également dans l'espace amoureux. Sex and the City regorge d'axiomes sur l'impossibilité des femmes à être trop libérées si elles souhaitent être prises au sérieux. L'environnement citadin encourage cette compétition pour deux raisons. Premièrement, du fait des « possibilités infinies » qu'évoque Carrie : les potentiels projets de vie qui s'offrent incessamment aux individus rendent plus difficile l'engagement exclusif. Deuxièmement, la surpopulation provoque un anonymat qui rend plus difficiles et plus risquées les rencontres. Tout un épisode de Sex and the City est ainsi dédié aux « freaks » rencontrés sur le marché amoureux. Comparant ces individus à des monstres de foire, Carrie liste les hommes bizarres avec qui elle est déjà sortie : un homme hurle sans raison sur la foule, un autre pourtant riche est kleptomane, un dernier est maladivement cupide. Ces individus apparaissent comme des « erreurs sur la marchandise » : ils promettaient d'être des princes charmants mais se révèlent être des crapauds. Enchâssée dans la compétitivité, se pose également, troisième problème, la question de l'attractivité. Les individus doivent s'évaluer en même temps qu'ils se construisent par rapport aux autres, ce qui est d'autant plus difficile que le travail identitaire est laborieux. Néanmoins, la valeur à laquelle ils s'estiment et la valeur qu'ils ont supposément « objectivement » déterminent le type de partenaire auquel ils peuvent prétendre. John Cage, l'ami d'Ally McBeal, explique qu'il est allé voir une prostituée car « En vérité, aller dans un bar pour trouver un coup d'un soir n'est pas une option pour moi : quelle que soit l'image qu'une femme se fait d'une nuit de passion, je suis pas dans la course ${ }^{8}$. » Sur le marché de l'amour, le romantisme n'est pas permis à tous : il est un idéal auquel tout le monde doit prétendre mais que 
peu peuvent réaliser. Enfin, un dernier problème est une tendance presque consumériste, que l'on pourrait juger influencée par le capitalisme (et qui l'est en partie) si elle ne portait pas une dimension féministe consistant à donner aux hérö̈nes des libertés jusqu'ici exclusivement masculines. Les hommes sont en effet testés, essayés, éprouvés - comme consommés à la chaîne par les héroïnes. Ils doivent affronter d'autres amoureux ou partenaires sexuels ainsi qu'un nouvel ethos féminin fait d'indépendance financière et émotionnelle, et sont pour la première fois massivement objectivés. Bon nombre des conquêtes de Sex and the City n'ont pas le luxe d'avoir un prénom, ce que l'interprète de Carrie Bradshaw, Sarah Jessica Parker, considère comme l'originalité de la série : « Je me sentais mal pour les hommes qui venaient jouer dans la série. [...] Ils n'avaient pas grand-chose à dire. Il y avait une brève présentation et puis le pantalon tombait !' » Curieusement, les représentations n'évoquent pas explicitement les jeux de capitaux esthétiques et économiques qui sous-tendent pourtant en profondeur les récits, que ce soit par touches (Ally McBeal refuse de sourire car cela provoque des rides) ou par schémas itératifs (Carrie Bradshaw n'a aucune relation sérieuse avec un homme déclassé, et le profil de son âme sœur en dit long sur les résidus patriarcaux : Mr Big est grand et robuste, il est plus âgé et plus riche qu'elle, et ill'appelle « ma petite »). Néanmoins, le déplacement des logiques économiques vers les rapports amoureux permet, dans ces séries, de symboliser la rationalisation qu'a apportée la démystification de l'amour, bien sûr, mais aussi de signifier la relativité et l'évolutivité de la « valeur » des individus. Le principe premier pour choisir son partenaire n'est plus la capacité de ce dernier à s'adapter aux fonctions que requiert le mariage, mais un ensemble de critères psychologiques, basés sur les attentes affectives.

\section{La sexualité, du soi au couple}

La sexualité passe ainsi du soi au couple, prenant appui sur des techniques de soi et l'expression amoureuse. En revendiquant une sexualité libérée, ces héroïnes portent une première attaque majeure contre les axiomes traditionnels tels que diagnostiqués par Gayle Rubin ${ }^{10}$. La société contemporaine porte en effet encore en elle les morales et les valeurs victoriennes de la fin du $\mathrm{XIX}^{\mathrm{e}}$ siècle, qui incitaient à la chasteté et incriminaient la prostitution, la masturbation ou encore l'avortement. Or, certaines de ces inquiétudes 
ont été absorbées par des branches féministes qui les ont confondues avec la domination masculine : ainsi, la pornographie, considérées parfois comme la théorie du viol, serait-elle le parangon de l'aliénation féminine. À ce premier paradigme féministe, qui voit la libération sexuelle comme une simple extension de privilèges toujours déjà masculins, Gayle Rubin en distingue un second, qui promulgue la liberté individuelle à disposer de son corps et de sa sexualité. Une telle compréhension des rapports épistémologiques entre sexualité et genre permet à Rubin de pointer les angles morts qui ont empêché la possibilité d'une théorie politique de la sexualité. Elle en énumère six axiomes : l'essentialisme sexuel naturalise les pratiques sexuelles (ce que Foucault avait déjà disqualifié dans son Histoire de la sexualité, ouvrage majeur dans le parcours intellectuel de Gayle Rubin); la négativité quant au sexe le qualifie de suspicieux voire dangereux; l'erreur d'échelle confère au sexe une « sur-signification »; l'axiologie des actes sexuels valorise certaines pratiques au détriment d'autres; la théorie domino des dangers sexuels, corollaire de l'axiologie, qualifie de bonnes ou de mauvaises les pratiques ; enfin, le manque d'un concept de variation bénigne de la sexualité empêche d'envisager la diversité des pratiques ${ }^{11}$. Elle défend, quant à elle, une définition de la sexualité selon « le degré de considération mutuelle, la présence ou absence de coercition et la quantité et qualité de plaisir qu'elle procure ${ }^{12} \gg$.

Sex and the City est la série la plus explosive vis-à-vis de ces résidus victoriens qui entérinent les interdits sexuels faits aux femmes. Les pratiques y sont nombreuses et hétérogènes, ce qui disqualifie tout essentialisme sexuel et apporte la variation bénigne défendue par Rubin; les héroïnes n’ont aucun remords ou regrets quant à leurs expériences, déconsidérant la négativité et la sur-signification ; enfin, le moralisme laisse place à des éthiques personnelles, qui sont moins régies par le rapport à la norme que par les envies personnelles: on peut faire ce que d'autres ne veulent pas faire mais il ne faut pas faire ce que l'on n'a pas envie de faire. Ces éthiques individualistes affaiblissent l'axiologie et la théorie domino des dangers sexuels. De façon moins radicale, Ally McBeal montre des efforts féminins pour conquérir la liberté sexuelle mais se heurte à des réaffirmations de la différence sexuelle émanant principalement des hommes. Richard, Billy ou même Larry opposent, dans des monologues particulièrement conservateurs, les instincts sexuels masculins aux envies affectives féminines. 
La dé-essentialisation sexuelle n'empêche donc pas une réification du genre, ce qui confirme la nécessité défendue par Gayle Rubin de ne les associer que stratégiquement. Selon elle, il faut en effet détacher l'étude de la sexualité de celle du genre, ce qui ne doit pas être compris comme une cassure mais comme une contextualisation : la relation entre sexualité et genre, entendue comme « situationnelle et non universelle », permet de voir les restrictions définitionnelles qui ont porté sur les pratiques sexuelles. Perce ainsi dans les années 1990 une vision globalement optimiste de la sexualité, à l'exception de Buffy, qui est régulièrement punie de faire l'amour (saison 2, saison 3), quand elle n'en est pas dépendante ou ne le regrette pas (saison 6). Cela est d'autant plus significatif que cette vision succède au backlash sexuel vécu par la société étasunienne dans les années 1980 à travers l'échec de l'Equal Right Amendment. La charge revendicative que regrettent les auteures critiques est bien présente dans le domaine sexuel : à bien des égards, Sex and the City peut être qualifiée d'anti-anti-sexe car les hérö̈nes défendent leur droit d'expérimenter et d'exprimer leurs rapports de désir-plaisir, transformant ainsi la sexualité en un moyen d'expression de soi et du couple. S'y formulent, de façon assez proche de la définition de Gayle Rubin, l'affection (ou non !) qui lie les partenaires, les envies et refus, variant selon les femmes, et bien entendu les satisfactions ou les insatisfactions qu'elles en tirent.

\section{Biopolitiques et technologies de soi à l'heure de l'émancipation féminine}

Plaisir charnel, épanouissement individuel, expression romantique, territoire de la démocratie relationnelle, partage intime... la sexualité recouvre, à partir des années 1990, des fonctions plurielles, tiraillées entre les techniques d'épanouissement personnel (dont témoignent les sex toys, par exemple) et l'idéal de l'amour. Elle agit à la fois au cœur de l'individu, qui souhaite désormais éprouver des plaisirs comblés et originaux, et comme centre de gravité de l'amour, qui ne se conçoit plus sans la chair : dans ces séries, toute relation amoureuse sexuellement insatisfaisante se termine mal. La vie sexuelle des héroïnes se produit sur deux axes. Leur volonté d'expérimenter des pratiques inédites témoigne de la charge identitaire, bien souvent tâtonnante, qu'elles investissent dans la sexualité : l'expression corporelle est une alternative à l'expression orale de l'émancipation. 

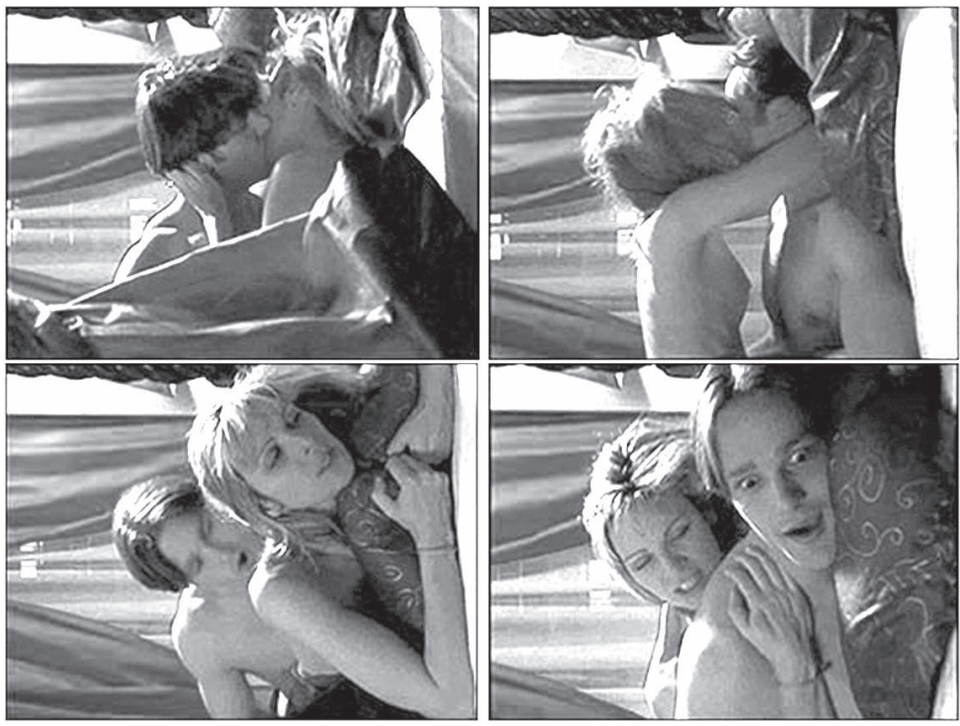

De plus, les héroïnes se permettent des comportements qui ont été, et qui continuent d'être parfois qualifiés de débauchés, ce qui agit comme une violente attaque contre la division opérée par l'hégémonie masculine entre femmes vertueuses et femmes amorales ${ }^{13}$. L'ordre hétérosexuel n'est pas épargné. Dès le quatrième épisode de la série, Samantha est montrée faisant l'amour avec un homme... en se plaçant derrière lui ${ }^{14}$. L'inversion est flagrante et la surprise qu'elle engendre tout autant : Samantha est proactive, une impression renforcée par un quart de tour de caméra qui laisse penser qu'elle est debout alors qu'elle est allongée, dans cette pratique qui est implicitement une pénétration anale (et qui implique qu'elle se soit dotée d'un phallus). L'expression de Samantha montre une énergie qui ne s'embarrasse pas de la douceur tandis que l'homme en question, sourcils levés, yeux écarquillés et bouche ouverte, apparaît des plus surpris mais pas dégoûté ni incommodé par les sensations ainsi octroyées.

Signe de la dénaturalisation de la sexualité mais de la résistance du système de genre, les technologies de soi orientées vers le plaisir féminin apparaissent. Dans un épisode resté célèbre, les héroïnes de Sex and the City découvrent le rabbit, sex-toy perfectionné. En plus d'être rose et flexible, le godemichet est surmonté d'un lapin dont les oreilles sont destinées à la stimulation clitoridienne. L'objet se détourne ainsi de la seule 
pénétration pour investir d'autres espaces de la sexualité féminine jusqu'ici délaissés. Le sex toy n'est plus un ersatz du phallus, symbole de l'absence masculine, mais une technologie de soi féminine, symbole du plaisir sexuel. La réaction de Charlotte incarne ce basculement : sa première réaction est un réductionnisme de la sexualité, qu'elle limite à la seule présence masculine (« je réserve le sexe pour quelqu'un que

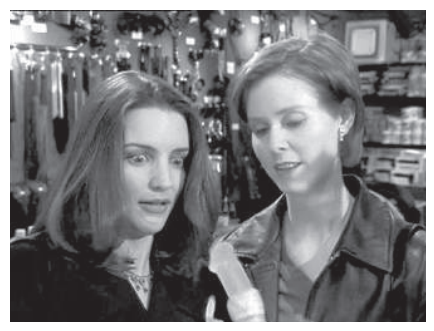
j'aime », dit-elle), ce qui semble être causé par la peur de découvrir sa propre sexualité (Charlotte redoutait que le vibromasseur ne soit « effrayant et bizarre »). Mais lorsque le vibromasseur s'avère esthétiquement féminin et supposément adapté à ses ressentis, Charlotte est enjouée : «Oh, c’est adorable ! Oh, je pensais que ça allait être effrayant et bizarre, mais non! C'est rose, pour les filles ! J'adore le petit lapin, il a une petite bouille, comme Peter Rabbit ! ${ }^{15} \gg$ Dans le même temps, une infantilisation du personnage est produite par la moue de son visage, le ton aigu de sa voix, les onomatopées qui ponctuent ses phrases et la référence à Peter Rabbit, personnage de bande dessinée pour enfants. La sexualité ainsi représentée est celle d'une féminité douce voire vulnérable. Cela n'empêche pas pour autant le plaisir : la suite du récit révèle que le « lapin »donne à Charlotte des orgasmes d'une intensité et d'une durée qu'aucun homme ne lui a jusqu'ici donnés. Mais Charlotte, devenue dépendante, craint de devenir en retour sexuellement indépendante du masculin. La sexualité autonomisée des femmes et la sexualité conjugale entrent en concurrence et demandent une hiérarchisation. C'est l'addiction de Charlotte envers le vibromasseur qui vient résoudre ce problème. Ses amies, inquiètes, interviennent finalement pour la priver du «lapin » car l'autonomie que tirait Charlotte de cette technique de soi se transforme en une forme d'asservissement et d'asocialité. Dans Ally McBeal, la substitution du masculin est plus sentimentale que sexuelle : l'héroïne n'a pas de vibromasseur mais une poupée gonflable prénommée (!) David, qu'elle partage avec sa colocataire Renée. L'usage qu'en fait Ally, contrairement à celui de Renée ${ }^{16}$, est néanmoins des plus chastes : peu de références sont faites à des pratiques sexuelles avec cette poupée, qui est d'ailleurs toujours habillée, et lorsque c'est le cas, il ne s'agit que de sous-entendus ${ }^{17}$. La thématique majeure rattachée à cet objet est plutôt celle de la compagnie. Elle est utilisée comme

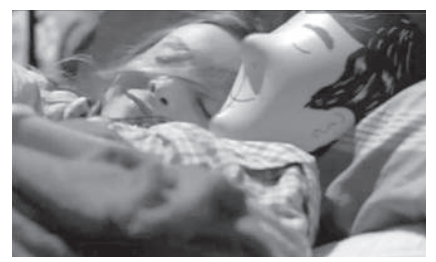


substitut du compagnonnage, le plus souvent pour dormir - c'est-à-dire pour rassurer l'héroïne dans l'état de vulnérabilité qu'est le sommeil, à la fois coulisses du monde social et territoire d'expression du subconscient. Les technologies de soi agissent ici non sur le registre du plaisir en vue d'accroître l'épanouissement sexuel, mais sur le registre de l'accompagnement en vue d'infléchir la solitude. Cette fonction devient d'ailleurs insupportable à Ally lorsque, après s'être séparée d'un homme qu'elle fréquentait, non car ils ne s'appréciaient pas mais parce qu'il a accepté un poste à Chicago, la poupée n'est plus substitut mais symbole de la solitude. Le lendemain matin de la rupture, Ally se force à sourire et à danser devant le miroir mais perd son sang-froid en trouvant la poupée dans son lit : elle l'attrape et la frappe violemment contre le lit puis le sol, avant de la faire exploser en s'asseyant dessus ${ }^{18}$. In fine, l'apparition de ces objets, dont la fonction oscille entre l'optimisme de la technologie de soi et le pessimisme de la béquille psychologique, apparaît toujours dans une comparaison, de concurrence ou de complémentarité, avec le masculin.

\section{La sexualité dans Buffy: \\ entre apogée de l'amour maudit et expérience queer}

Il faut néanmoins souligner une face sombre de ces représentations largement positives : la série télévisée Buffy se distingue des autres productions par un relatif pessimisme quant aux relations amoureuses et une négativité poussée à propos de la sexualité, et ce quels que soient les partenaires de l'héroïne. Une première de ses histoires se déroule avec Angel, un vampire à qui des gitans ont rendu son âme après qu'il eût tué l'une des leurs. Condamné à porter sur sa conscience les meurtres de son alter ego sanguinaire Angelus, Angel incarne tout à la fois la figure du bad boy, du torturé et du rédempteur. Sa relation avec Buffy porte de nombreux marqueurs romantiques : leur amour est salvateur mais il est maudit, il se construit contre les structures existantes et il réinsère la figure tutélaire du masculin dans l'empowerment de la super-héroïne. Angel s'est en effet promis de protéger la Tueuse : son nom angélique n'est pas anodin et sa fonction, dans Buffy comme dans sa série dérivée Angel, est bien souvent de défendre la Tueuse, même si elle n'en a pas toujours besoin ${ }^{19}$. Ce patriarcat que subit Buffy est mis en exergue 
dès la première saison, lorsqu'elle perd sa virginité avec Angel. Au cours d'un double épisode intitulé « Innocence », peu après qu'ils se sont symboliquement mariés (Angel offre solennellement une bague à Buffy), le couple fait l'amour. Les quelques images auxquelles le spectateur a accès se déroulent sur fond d'une musique douce et de gémissements surtout féminins et ne montrent que des peaux nues en gros plans ou une insistance sur la bague de Buffy. La scène se clôt sur un « je t'aime » d'Angel, qui enchaîne brutalement avec le visage vampirique d'Angelus : au moment de l'orgasme, Angel vit un moment de pur bonheur, ce qui lui était interdit par le sort des gitans sous peine de perdre son âme. Redevenu Angelus mais se faisant passer pour Angel, il rompt très violemment avec Buffy, moquant sa naïveté, décrivant son romantisme comme une croyance en « des cloches qui sonnent, des feux d'artifice et un chœur suave de jolis petits oiseaux », enfin critiquant sa performance physique.

Joss Whedon lui-même, créateur de Buffy, auteur et réalisateur de cet épisode, considère cette séquence de rupture comme l'une des plus significatives de la série en ce qu'elle cristallise les inégalités hommes-femmes : «C'est l'une de mes scènes préférées, qui représente quelque chose de plus global. La scène où Angel devient Angelus mais prétend qu'il ne l'est pas, qu'il a juste eu un coup d'un soir avec Buffy, et que ça la détruit. Il va vers elle et lui dit "Pourquoi t'en fais toute une histoire ?". Il se comporte comme un mec. J'ai écrit cette scène et je me suis dit "Je dois être plus affreux que je ne l'ai imaginé parce que je suis capable d'écrire ça." Je crois que j'ai puisé dans quelqu'un d' horrible, et c'est venu assez facilement ${ }^{20}$. $\gg$ Cet arc narratif s'inspire évidemment du décalage supposé entre de jeunes femmes qui croient en l'amour et des hommes qui ne seraient intéressés que par la sexualité, s'enfuyant sitôt l'acte accompli. L'amour maudit, thématique du romantisme classique, est ainsi coordonné avec les inégalités genrées des pratiques, et rendu plus violent encore par le bon droit qui entoure l'acte sexuel entre Buffy et Angel (leur amour bien sûr, mais aussi leur mariage symbolique que rappellent des plans insistants sur la bague de l'héroïne). Une lecture traditionnelle de l'épreuve que traverse Buffy est donc encouragée par le cadre quasi marital qui légitime l'acte sexuel, par ses conséquences dévastatrices pour Buffy et par le peu de ré-ordonnancement des genres (le féminin est émotif, le masculin est sexuel). Le ton dénonciateur de Joss Whedon, tant dans ce qu'il dit de cette séquence que 


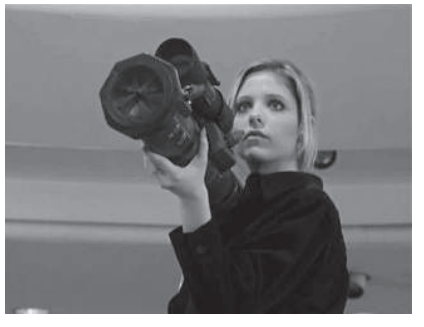

dans les modalités représentationnelles de l'épisode (le téléspectateur compatit évidemment avec Buffy), témoigne d'un féminisme qui perçoit la sexualité comme négative, un territoire masculin où le féminin souffre forcément.

Dans Buffy, la sentimentalité des femmes est leur talon d'Achille : plus tard dans l'épisode, Angelus confie à un acolyte « Elle est plus forte que n'importe quelle Tueuse que tu as rencontrée, la force n'y parviendra pas. » Pointant son cœur, il ajoute : «Il faut travailler de l'intérieur : pour tuer cette fille, il faut l'aimer. » La capacité d'agir si forte de Buffy dans l'espace public semble s'effacer dans le domaine amoureux, où elle est laissée face à un romantisme aussi fusionnel qu'impossible. Buffy est affaiblie par une sexualité strictement contenue au cadre romantique. La résolution opère finalement, comme chez les trentenaires citadines, en déplaçant les compétences obtenues dans la sphère publique vers la sphère privée : Buffy décide de tuer Angelus. Sa vengeance n'est pas sans empowerment puisqu'elle part à sa recherche armée de son éternel pieu, symbole phallique, mais aussi d'un... bazooka. La taille de l'instrument, que Buffy utilise rarement dans la série, n’est évidemment pas sans ironie. Néanmoins, ne pouvant se résigner à tuer Angelus, elle se contente temporairement de représailles sexuelles en le frappant dans l'entrejambe et le laissant ainsi agenouillé devant elle. Buffy devient d'autant plus dangereuse qu'elle est désormais femme, et femme blessée de surcroît ${ }^{21}$. Avant d'entamer ce qui sera sa troisième grande histoire amoureuse (avec un autre vampire nommé Spike), Buffy connaît quelques autres relations notables qui ne font que renforcer la négativité qui entoure la sexualité dans la série : arrivée à l'université, elle fait la rencontre d'un jeune homme normal avec qui elle fait l'amour, mais celui-ci la quitte aussitôt après, rejouant le drame de sa première fois. La sexualité reste un vecteur d'oppression. Elle entame ensuite une relation avec Riley, un humain paramilitaire engagé dans la lutte contre les vampires. Joss Whedon lui-même reconnaît l'échec de cette relation, trop lisse pour évoquer les problèmes contemporains de l'amour : « Le problème qu'on a eu avec Riley [c'est qu'on] s'est dit, "donnons à Buffy une relation saine”, et le public n'en a pas voulu. Ils faisaient un super boulot ensemble mais, en même temps, quand ils étaient heureux, ça rendait les gens fous [parce que], au final, l'histoire du grand amour ne peut pas être un long 
fleuve tranquille ${ }^{22}$. » À l'incarnation contemporaine de James Dean que représentait Angel, bad boy insaisissable, le personnage de Riley rappelle Gary Cooper, militaire héroïque et moral. Leur histoire se termine sur une note là encore patriarcale, ayant trait à la fonction protectrice du masculin rendue obsolète par la force de la Tueuse : Riley quitte Buffy, et la ville !, car il ne supporte pas d'être plus faible qu'elle. Comme pour rassurer sa masculinité, le personnage rejoint l'armée officielle, non sans s'être au préalable engagé dans une relation malsaine avec une vampire en vue de comprendre l'amour de Buffy pour Angel. L'échec de leur relation incombe donc au masculin humain, incapable de supporter la supériorité physique de sa compagne ni sa capacité à communiquer avec un autre monde que celui des humains. Enfin, la troisième histoire de la Tueuse est également avec un vampire prénommé Spike, et est construite en négatif de l'amour romantique pur mais maudit qu'elle entretient avec Angel. Cette relation commence au début de la sixième saison, alors que Buffy, décédée lors de l'épisode final de la saison 5, est ressuscitée par ses amis : elle revient à la vie dans son cercueil et doit s'extraire elle-même de la terre. L'héroïne vit ainsi une renaissance similaire à celle qu'expérimentent les vampires qu'elle a toujours combattus, rejoignant leur monde. Les frontières entre le monde humain que la Tueuse doit protéger et le monde démoniaque qu'elle combat étaient déjà rendues floues par sa relation avec Angel, mais elles sont définitivement écrasées à compter de cette sixième saison : la Tueuse est devenue non-humaine. Même si Tara, son amie magicienne, la rassure quant à sa normalité physiologique ( « Tu es différente, te déplacer de là où tu étais, ramener ton essence dans ton corps, ça t’a altérée au niveau moléculaire [...] mais c'est superficiel, ça n'a pas plus d'effet qu'un mauvais coup de soleil. »), l'expérience psychologique du retour à la vie fait basculer Buffy dans un entre-deux : la Tueuse est étrangère, rendue queer, au monde humain. Elle est bien entendu traumatisée, d'autant qu'elle a été arrachée au paradis, mais ne peut partager son mal-être avec ses amis puisqu'ils sont responsables de son état. L'héroïne voit alors en Spike un interlocuteur privilégié, un non-humain qui peut la comprendre. De nombreuses recherches ont souligné le potentiel queer de Spike : pour Dee Amy-Chinn « Son genre et ses transgressions sexuelles lui donnent des perspectives et des capacités qui ne sont pas possibles pour les autres membres du Scooby Gang (surnom 
donné au groupe qui entoure Buffy dans ses missions surnaturelles), qui sont contraints à des identités genrées plus rigides ${ }^{23}$. »

Buffy et Spike entretiennent une relation sadomasochiste qui explicite les rapports de pouvoir à l'œuvre dans la sexualité, à rebours de la douceur expérimentée par Buffy avec Angel, et de l'éthique romantique qu'incarnait Spike de son vivant, poète raté avant d'être vampire. Le sadomasochisme permet à Buffy d'exprimer son identité nouvellement queer et la sexualité devient un défouloir où formuler ce qui ne peut être dit en public : coulisses d'une identité marginale, il n'est d'ailleurs pas étonnant que Buffy se confie d'abord sur cette relation à un personnage lesbien, Tara. Les pratiques sexuelles qu'a Buffy avec Spike tranchent donc radicalement avec l'hétéronormativité de ses précédentes relations. À la position du missionnaire qui plaçait l'hérö̈ne innocente sous le large torse d'Angel ou de Riley, succèdent des combats avec Spike pour la domination. Ces représentations sadomasochistes ne sont pas apologiques : le moteur de la relation entre Buffy et Spike n'est pas l'amour ni la bienveillance, mais l'envie de contrôle et le besoin d'oublier son désarroi. Le récit amalgame sadomasochisme et absence de care lorsqu'il montre Buffy rossant violemment Spike, ce qui est loin de correspondre à la réalité des pratiques sociales comme l'a bien montré Gayle Rubin ${ }^{24}$. Buffy elle-même dit à répétition le dégoût que lui inspirent leurs pratiques. Lorsqu'elle rompt finalement, elle signifie sa volonté de revenir dans le monde des humains en appelant son partenaire, non par son prénom de vampire Spike, mais par le prénom qu'il portait dans sa vie d'humain : «Au revoir, William ${ }^{25}$. » La communication entre les deux mondes est ainsi rompue, l'ordre oppositionnel entre Tueuse et Vampire est réaffirmé et les pratiques sexuelles alternatives renvoyées au monde du non-humain. Dans la série, l'explicitation des politiques identitaires et amoureuses rend les relations perverses, corrompues par un pouvoir qui semble n'être que négatif. En la matière, le zénith de cet arc narratif est certainement la scène très controversée où Spike tente de violer Buffy ${ }^{26}$ car il ne comprend pas qu'elle veut sincèrement rompre : la sexualité est hors de contrôle, hors de tout échange langagier et ramenée à une pulsion possessive. La particularité de ces représentations est que Buffy n'assume aucunement son statut de non-humain ni le rapport désir-plaisir qu'elle tire de la mise en scène du pouvoir par la sexualité sadomasochiste. Une forme de moralisme pèse sur elle, qui l'empêche d'affirmer une identité instrumentalisant un 
pouvoir sexuel déviant de la féminité classique. À l'heure où la quadragénaire Samantha Jones (Sex and the City) est montrée derrière un partenaire sexuel, à l'heure où la trentenaire Ally McBeal performe une mascarade de la sexualisation féminine, il apparaît impossible pour la plus jeune Buffy de jouer avec les codes de la sexualité féminine à moins de s'en détester. L'une des raisons se niche certainement dans les publics visés par ces productions : Buffy, diffusée sur The WB, avait pour objectif, de l'aveu de la présidente des programmes Susanne Daniels, d'attirer un public jeune tout en convainquant les critiques ${ }^{27}$.

Dans l'histoire des héroïnes de télévision, Buffy a été très importante : contrôlant ses pouvoirs, tuant des dizaines de vampires chaque semaine, sauvant le monde à chaque saison, la femme menue et blonde tant stigmatisée devient une jeune fille à la force physique extraordinaire capable de suivre ses études la journée et d'arpenter les cimetières le soir. Buffy peut douter mais elle n'a pas peur, ni de sa mission, ni de ses ennemis, ce qui en fait un pivot crucial dans les représentations de l'empowerment féminin. Dans la sphère privée en revanche, la Tueuse apparaît, comme bon nombre de super-héroïnes de la culture populaire, dépassée par son pouvoir, en l'occurrence sexuel. La sexualité agit à double tranchant, toujours coupant pour Buffy : soit elle est l'expression d'un romantisme qui rend fou le masculin et qui punit le féminin, soit elle est une théâtralisation perverse des rapports de pouvoir et des politiques identitaires dans l'amour. Les capacités d'agir sexuelles de Buffy sont tour à tour laminées par l'amour maudit ou rendues dangereusement incontrôlables par la sexualité plastique sadomasochiste. 


\section{Notes}

1 Bozon, Michel, Leridon, Henri, « Les constructions sociales de la sexualité », Populations, 5, 1993, p. 1173-1195.

2 Ally McBeal, saison 2, épisode 4.

3 Ally McBeal, saison 3, épisode 1.

4 Sex and the City, saison 3, épisode 6.

5 Sex and the City, saison 1, épisode 4.

6 Sex and the City, saison 1, épisode 1.

7 Sex and the City, saison 1, épisode 7.

8 Ally McBeal, saison 1, épisode 2.

9 Documentaire « The United States of Television: America in Primetime $\gg$, épisode 3, BBC.

10 Rubin, Gayle, « Thinking Sex: Notes for a Radical Theory of the Politics of Sexuality », in Vance, Carole S., Pleasure and Danger: Exploring Female Sexuality, Londres, Routledge, 1992.

11 Ces variations bénignes ont pourtant été révélées par les célèbres rapports Kinsey, étude empirique sur les comportements sexuels masculins (produite en 1948) et féminins (produite en 1953). Voir Kinsey, Alfred, Sexual Behavior in the Human Male, Ishi Press, 2010, Kinsey, Alfred, Sexual Behavior in the Human Female, Ishi Press, 2010.

12 Rubin, Gayle, ibid.

13 Giddens, Anthony, Pierson, Christopher, Conversations with Anthony Giddens. Making Sense of Modernity, Palo Alto, Stanford University Press, 1998, p. 123.

14 Sex and the City, saison 1, épisode 4.

15 Sex and the City, saison 1, épisode 9.

16 Tracey Owens Patton souligne l'usage racialisé de cette poupée. Si Ally ne l'utilise que pour dormir, sa colocataire afro-américaine Renée la sexualise bien plus, allant jusqu'à s'intéresser à la taille de son pénis. La distinction entre une féminité blanche innocente et une féminité noire très sexualisée est ainsi réaffirmée. Owens Patton, Tracy, « Ally $\mathrm{McBeal}$ and her Homies. The Reification of White Stereotypes of the Other », Journal of Black Studies, 32, 2, 2001, p. 229-260. 
17 Dans Happy Birthday, Baby, Ally demande à un homme qu'elle fréquente de monter boire un dernier verre chez elle. Celui-ci refuse. Frustrée, Ally embarque la poupée avec elle dans sa chambre mais sans que l'on sache si la visée est sexuelle ou non.

18 Quelques épisodes plus tard, le téléspectateur découvre que Ally a remplacé sa poupée.

19 Dans un épisode resté célèbre, « I Will Remember You », Angel redevient humain, ce qui lui permet de vivre une vie normale avec Buffy. Mais le déséquilibre de leurs pouvoirs lui devient très rapidement insupportable, l'amenant à demander à des forces supérieures de lui rendre ses capacités surnaturelles afin qu'il puisse protéger la Tueuse. Angel préfère pouvoir protéger Buffy plutôt que de vivre une histoire amoureuse avec elle.

20 Lavery, David, Burkhead, Cynthia, Joss Whedon: Conversations, Jackson, University Press of Mississippi, 2011, p. 78.

21 Au matin, lorsqu'elle rentre chez elle, sa mère la trouve changée sans savoir ce qu'il s'est passé durant la nuit. Le récit signifie ainsi que la perte de la virginité métamorphose les femmes physiquement, insinuant des répercussions importantes.

22 Lavery, David, ibid., p. 73.

23 Amy-Chinn, Dee, «Queering the Bitch: Spike, Transgression and Erotic Empowerment », European Journal of Cultural Studies, 8, 3, 2005, p. 313-328.

24 Rubin, Gayle, ibid., p. 267-293.

$25 B u f f y$, saison 6, épisode 15.

26 Buffy, saison 6, épisode 19.

27 Jacobs, A.J., « Interview with a Vampire Chronicler », Entertainment Weekly, 25 avril 1997. Disponible en ligne : http://www.ew.com/ew/ article/0,,287570,00.html, consulté le 14 septembre 2014. 



\section{Le monde post-romantique des héroïnes quadragénaires (2004-2014).}





\section{Détraditionnalisation, découverte du féminisme et urgence de nouveaux liens amoureux}

\section{Une émancipation malgré elles}

Au premier abord, on pourrait penser que la période 1997-2004 a été une enclave dans l'histoire de la fiction télévisée étasunienne. Sex and the City, Ally McBeal ou Alias achevées, aucune héroïne ne semble avoir repris explicitement l'étendard du féminisme. Toutefois, les femmes n'ont pas complètement disparu du petit écran, même si leur succès critique et public est incontestablement plus modeste. À partir de 2004, ce sont les quadragénaires qui intéressent la fiction télévisée : The Good Wife, Desperate Housewives ou Weeds, bien sûr, mais aussi Cougar Town, United States of Tara, The Big C, The New Adventures of Old Christine, Nurse Jackie, Private Practice, Enlightened, Happily Divorced, Dirt, The Comeback, Web Therapy ont toutes pour personnage principal une femme d'une quarantaine d'années. Pour la plupart d'entre elles, elles subissent, comme les trentenaires de la décennie précédente, la résistance de normes patriarcales tout en luttant pour s'imposer dans la sphère professionnelle. Mais cette fois-ci, toutes ont été mariées et la plupart sont séparées, divorcées ou veuves avec enfants. Ce faisant, elles ont un avantage certain sur leurs prédécesseures : elles ont accompli les attendus sociaux (mariage, enfants) et rassuré la norme hétérosexuelle.

Leurs histoires portent des ressemblances frappantes avec les héroïnes trentenaires, tout en se distinguant de leur trajectoire : à la trentaine, c'està-dire à l'âge d'Ally McBeal ou de Carrie Bradshaw, elles n'expérimentaient pas la relation pure en cherchant le Prince charmant mais étaient mères au 
foyer dans des banlieues souvent huppées. Dès vingt-cinq ou trente ans, ces hérö̈nes vivaient le paradoxe de l'amour romantique : femmes mariées et mères heureuses, elles étaient enfermées dans des rôles genrés. Lorsque nous commençons à suivre leurs histoires, elles sont propulsées hors du monde marital et romantique, généralement du fait d'un bouleversement dans la vie de leur mari. Après les scandales financiers et sexuels de son époux (dont les ébats filmés avec une prostituée sont rendus publics), Alicia Florrick est contrainte de retourner travailler pour subvenir aux besoins du foyer (The Good Wife). Christine Campbell découvre que son ex-mari, avec lequel elle s'entend au demeurant très bien, a une nouvelle petite amie nommée Christine : l'écart d'âge et l'homologie de leurs prénoms amènent leur entourage à les surnommer respectivement « Vieille Christine » et « Jeune Christine », pointant l'inégalité de genre devant le statut de quadragénaire divorcé.e (The New Adventures of Old Christine). Après dix-huit ans de vie commune, Fran Lovett apprend de son mari qu'il est homosexuel (Happily Divorced). À la mort de son mari, Nancy Botwin refuse de diminuer ses dépenses et devient dealeuse de drogue pour combler les déficits du budget familial (Weeds). D’autres héroïnes doivent affronter des maladies ou la dépendance, qui ont des répercussions fortes sur leur mariage : dans The Big C, Cathy Jamison décide, après avoir découvert qu'elle est atteinte d'un cancer, de se séparer brièvement de son époux pour fuir l'étouffement de la vie domestique avant de repenser son couple; dans Nurse Jackie, l'héroïne infirmière, dépendante des analgésiques, entretient une relation extra-conjugale avec le pharmacien de son hôpital, confondant dans une même personne les fonctions d'amant et de fournisseur. Invariablement, est évoquée l'humiliation qu'engendre l'effondrement du rêve américain. Les héroïnes perdent la face, subissant les conséquences d'actes qui ne sont souvent pas les leurs. L'écroulement du rêve américain révèle les comportements abusifs de l'entourage et les conséquences de cette exigence d'un amour sacrificiel. Cela amène les héroïnes à prendre conscience - thématique classique du féminisme radical - des inégalités structurelles qui pesaient et continuent de peser sur elles : les disparités économiques du mariage de la première modernité restreignent leurs capacités d'agir tandis que les inégalités communicationnelles de l'amour deviennent de moins en moins tolérables. Le néoféminisme, si influent auprès des héroïnes trentenaires, devient un ressort comique produit aux dépens des héroïnes qui le 
vivent : jusque dans les titres, la « cougar » Jules Cobb (Cougar Town) ou la « vieille 》 Christine (The New Adventures of Old Christine) incarnent le problème de l'obsolescence de ce mouvement féministe passé quarante ans. Le retour à la sphère privée, qui peut sembler être un backlash, est en fait un réinvestissement par les héroïnes d'un territoire auquel elles ont été confinées mais qu'elles n'ont que peu souvent défini. C'est certainement ce qui explique que la séparation soit dédramatisée : la liberté qu'elle redonne est comprise au miroir des contraintes du mariage. Un divorce réussi devient ainsi préférable à un mariage raté, le succès ou l'échec dépendant des aspirations individuelles et de leur harmonisation dans le couple. Dès le pilote de The New Adventures of Old Christine, alors que son frère lui fait remarquer « qu'elle et [son ex-mari] ont un divorce bizarre », l'héroïne répond : « Il n'est pas bizarre, il est génial : mon divorce est bien meilleur que la plupart des mariages ${ }^{1}$. » Fran Drescher, productrice et héroïne de plusieurs séries télévisées, crée en 2011 une sitcom au titre explicite : Happily Divorced $^{2}$. Dès le pilote là encore, Fran encense la séparation « Le divorce, c'est le nouveau mariage ! ${ }^{3}{ }^{\prime}$

La deuxième vie de ces femmes tardivement émancipées, qui pour certaines reviennent au célibat après quinze ans de mariage traditionnel et une rupture marquante, est au cœur de la fiction depuis le milieu des années 2000. Les héroïnes n'interrogent pas l'après-féminisme comme les «nouvelles "nouvelles femmes" » mais l'après-romantisme. Profondément déçues par l'amour romantique qui n'a su tenir ses promesses, elles découvrent qu'il est possible de créer du lien amoureux autrement que par la famille nucléaire. Elles tentent ainsi d'accomplir des relations pures et des reformulations inédites du privé et du public. Le choc représenté et la rapidité avec laquelle leur existence en est ébranlée rappellent que la génération $X$ est hétérogène, certaines femmes étant héritières des avancées du féminisme (comme les trentenaires de Sex and the City et $A l l y M c B e a l)$, d'autres ayant vécu la révolution sexuelle et ayant vu le patriarcat concurrencé par la montée des revendications égalitaires (comme le font les quadragénaires qui peuplent la fiction télévisée à partir de 2004). La chaîne câblée Showtime est, seule, à l'origine de plusieurs héroïnes innovantes: Weeds, Nurse Jackie, United States of Tara ou encore The Big C. Une telle systématicité résulte bien sûr d'une stratégie de programmation visant à concurrencer $\mathrm{HBO}$ dont les grilles de programme 
ont, dans les années 2000, délaissé les personnages féminins. De l'aveu même du président de la programmation Michael Lombardo, la chaîne a été « effrayée par sa propre réussite: Les Sopranos et Sex and the City étaient de tels succès qu'il y a eu ensuite une inertie dans l'innovation ${ }^{4} \gg$. Si HBO a en effet marqué l' histoire des héroïnes télévisuelles avec Sex and the City, elle ne comporte en 2005, au lancement de Weeds, qu'une seule série centrée sur une femme. The Comeback, dont l'héroïne est une actrice has-been sur le retour, est produite par Lisa Kudrow et le créateur de Sex and the City, Michael Patrick King. Loin de proposer, à la manière de Showtime, une femme forte au-devant d'obstacles post-romantiques, The Comeback montre les constantes humiliations auxquelles consent Valerie Cherish (et contre lesquelles elle tient certes bon) pour rester sous les projecteurs. The Comeback ne fut pourtant pas le seul projet de $\mathrm{HBO}$ centré sur une femme : de nombreuses autres séries, tuées dans l'œuf, témoignaient de l'intérêt de la chaîne pour les problèmes féministes (Women's Studies, Washingtonienne, Tilda, un dernier projet voulait s'inspirer de Gloria Steinem). En parallèle de cette désertion féminine et féministe, Show time voit dans le topos de l'héroïne déboussolée l'opportunité de concurrencer $\mathrm{HBO}$ tout en se permettant des représentations subversives car affranchies à la fois de la censure et des directives publicitaires, comme toute chaîne câblée disponible exclusivement par abonnement. Le directeur général de Showtime, Matt Blank, parle explicitement de cette ligne éditoriale. Évoquant la mise à l'honneur des nouvelles subjectivités féminines (que ce soit auteures, interprètes ou personnages), il déclare au Daily News : « Nous sommes tout à fait conscients de l'opportunité que nous avons de faire les choses différemment de ce que les autres peuvent faire à la télévision et au cinéma, et nous aimons à penser que nous en tirons tout profit $^{5}$. » La stratégie est payante puisqu'en 2009, Showtime obtient plus de récompenses que n'importe quelle autre chaîne, en plus d'augmenter considérablement son nombre d'abonnés ${ }^{6}$.

Pour Andrea Press, la raison de ce succès public et critique tient à ce que les séries de Show time visent au-delà d'un public exclusivement féminin en élargissant les problèmes rencontrés : il ne s'agit plus seulement d'amours précaires, de sexualité plastique, d'après-féminisme ou de conflits hommes/ femmes, mais de famille, de travail, de finances, de troubles identitaires ou d'addictions, autant de thèmes qui « dépassent ce qui a traditionnellement 
été considéré comme des problèmes de femmes », sans néanmoins renier le point de vue féminin. S'il s'agit en effet, par exemple, de dépendance à la drogue, « ce n'est pas raconté dans la perspective de The Wire ou de la vie urbaine d'un tueur à gages : on est en train de voir une infirmière respectable, une femme au foyer, ce qui est d'autant plus fascinant ${ }^{7} \gg$. Les auteures de ces séries (Weeds, Nurse Jackie, The Big C, United States of Tara) sont toutes des femmes, qui ont pour la plupart exprimé le désir de raconter les difficultés de la subjectivité féminine : la co-créatrice de Nurse Jackie reproche ainsi aux « histoires de mecs de raconter des conquêtes - obtenir un boulot, gagner les jeux Olympiques, peu importe - alors que les histoires de femmes ne sont pas de l'ordre de l'apogée ${ }^{8} \gg$. À l'instar de Roseanne Barr, dont l'envie de créer une héroïne « normale » venait d'une frustration quant aux monstrations idéalisées des mères au foyer, Liz Brixius confie avoir voulu prendre à contre-pied la surreprésentation donnée aux médecins dans les séries médicales et privilégier le point de vue d'une infirmière. La spécificité des auteures féminines est par ailleurs soulignée par les interprètes principales, à l'instar d'Edie Falco (Nurse Jackie) : « Je ne suis pas habituée à pouvoir avoir une opinion sur ce que mon personnage devrait faire ou qui elle devrait être, mais [Brixius et Wallem] n'arrêtent pas de me dire "donne ton avis". Elles sont sensibles, vulnérables et intelligentes. Ce sont de vrais modèles pour une femme ${ }^{9}$. $>$ La publicisation du genre des auteures et de la prérogative donnée à la perspective féminine dans les récits est un indice de la stabilisation des contre-publics subalternes féminins au cœur les industries télévisuelles.

L'attrait scientifique de ces séries repose notamment sur les discordances hétéroclites que vivent les héroïnes avec le nouveau monde dans lequel elles évoluent, qu'elles n'ont pas choisi mais dont elles découvrent les avantages. À mi-chemin entre le drame et la comédie, le ton de ces « dramédies » est idéal pour décrire le balancement entre les attentes résiduelles de la première modernité et l'incapacité désormais connue de les tenir. La collision de la première et de la deuxième modernité crée des révoltes du féminin contre le masculin, interroge la manière de faire lien amoureux sans y être consumée, montre des crises de nerfs de femmes bourgeoises qui ne sauvent plus les apparences. Les héroïnes réalisent à postériori l'inégalité structurelle dans laquelle elles étaient plongées et qui, en plus de leur être défavorable, servait en réalité leur époux. Ainsi, la dureté de Cathy Jamison tranche avec 
le care dont elle a fait preuve jusqu'ici ; l'égocentrisme absurde de Christine Campbell est le symptôme d'une incertitude identitaire ; la détermination parfois irréfléchie de Nancy Botwin montre l'écart entre ce qu'elle veut et ce qu'elle peut obtenir ; plus largement enfin, les innombrables crises des Desperate Housewives (addictions en tous genres, dépressions, violences...) viennent de l'incapacité non seulement de vivre la première modernité, mais surtout d'en tenir la représentation. La scène désormais célèbre où, après avoir été violemment critiquée par son époux, Bree Van de Kamp (Desperate Housewives) fond en larmes dans la salle de bains avant de ressortir plus impeccable qu'elle n'y est entrée, est annonciatrice de la dépression du personnage. Durant la série, Bree est sans conteste la femme qui, le plus, se perd dans l'addiction et pâtit de l'obsolescence de son ethos ${ }^{10}$. Les ressorts psychologiques continuent donc d'influencer les représentations, mais autrement que par la sensibilité expressiviste de la décennie précédente. Les émotions, qui étaient le moteur de la réflexivité des trentenaires, s'expriment ici de façon erratique et surtout imprévisible - un comble pour des héroïnes qui sont priées d'être de parfaites gestionnaires. Colères, frustrations et impétuosités féminines explosent (enfin !) d'avoir été trop tendues, trop contenues, trop éprouvées, ce qui pose la question de la représentation de soi qu'il est épuisant de donner. La fin du surmoi féminin apporte des moments qui se veulent jouissifs, où les héroïnes se moquent de l'image qu'elles renvoient d'elles-mêmes, balayent les règles de bonne conduite et entendent désormais se comporter comme elles le souhaitent : le contraste est d'autant plus fort et amusant qu'elles étaient soumises à l'ethos particulièrement contraignant du rêve américain et de ses banlieues.

\section{Les responsabilités sont-elles solubles dans les psychotropes? Culpabilités féminines et phénomènes compensatoires}

Symboles des difficultés qu'ont les femmes à maintenir seules la santé émotionnelle et financière du foyer avec la représentation publique du rêve américain, une recrudescence de psychotropes, drogues et autres produits aux vertus sédatives ou excitantes apparaît : Jackie Peyton est dépendante 
d'analgésiques (Nurse Jackie), Cathy Jamieson soulage ses douleurs avec du cannabis (The Big C), et de façon très répandue, le vin rouge devient un rituel d'apaisement, quand il n'est pas comme dans Cougar Town une addiction collective rendue amusante par l'énormité de la consommation (Jules utilise des vases pour verre à vin). Le thème de l'addiction ne date pas du milieu des années 2000 ni n'est l'exclusivité des héroïnes déboussolées de Showtime. Dès les années 1980, Murphy Brown est explicitement dépendante de l'alcool et des médicaments tandis que Roseanne a, trait typiquement populaire, un rapport compulsif à la nourriture. Néanmoins, le sujet prêtait tout au plus au sarcasme et non à une mise en scène systématiquement drôle. Or, à compter de 2004, alcools, médicaments ou drogues participent du comique des burn-outs féminins en ce qu'ils encouragent des comportements à rebours de l'identité portée par les héroïnes. Ivre, Bree van de Kamp rejette son puritanisme sexuel mais pas son ethos bourgeois ; c'est en uniforme d'infirmière et dans les toilettes de l'hôpital que Jackie Peyton broie des pilules de Vicodin pour en consommer des rails; les shots de tequila que la «bonne épouse » Alicia Florrick boit cul sec la rendent moins austère.

Ce décalage entre deux identités, l'une respectable et l'autre à la lisière de la folie, met à jour l'hypocrisie de la représentation du rêve américain à l'heure de l'explosion des revendications égalitaristes dans le couple. Face à la crise du devoir, les héroïnes utilisent les médicaments comme des béquilles sur un mode qui se rapproche, comme l'a identifié Alain Ehrenberg, du dopage ${ }^{11}$, une analogie qui est d'ailleurs mobilisée dans un arc narratif de Desperate Housewives ${ }^{12}$ durant lequel Lynette, ayant accepté de coudre les costumes du spectacle de ses enfants afin de s'intégrer parmi les mères modèles de l'école, apprend de l'une d'elles que les médicaments contre les troubles de l'attention amplifient l'énergie. À bout de nerfs car ne parvenant pas à mener à bien ses projets, Lynette abandonne ses principes et ingère les médicaments. L'effet est quasi immédiat : de retour parmi les mères modèles, l'héroïne est coiffée, maquillée et son assurance tranche avec sa précédente fébrilité. Féminité et maternité sont regagnées en même temps que culpabilité et insuffisance sont évacuées. L'arc se clôt deux épisodes plus tard lorsque, épuisée par le vacarme des enfants en train de jouer, Lynette rêve au suicide ${ }^{13}$. Quoiqu'avec des conséquences moins tragiques, le vin rouge se diffuse tout autant dans les 
représentations médiatiques, venant sous-tendre la désinhibition globale des héroïnes de cette décennie tout en participant de la surreprésentation des classes sociales supérieures. Le vin rouge agit en effet comme un symbole distinctif, spécifique aux héroïnes dont les histoires se déroulent à nouveau dans la sphère privée, loin des cocktails d'Ally McBeal et de Sex and the City (où le cosmopolitan insistait sur la féminité urbaine) ou de l'addiction à la caféine de la très indépendante Lorelai Gilmore (Gilmore Girls). The Good Wife montre très régulièrement son hérö̈ne en apprécier un verre : le spectre de sa consommation va de la délectation des arômes et du goût, jusqu'au jeu avec le contenant, en passant par des formes plus dépressives, quand l'héroïne le boit en une seule gorgée, perd son regard dans le verre ou attend nerveusement que l'horloge marque 17 heures pour pouvoir décemment ouvrir une bouteille. Cette fonction anxiolytique est signifiée de façon plus flagrante encore dans les sitcoms où les verres remplis à ras bord sont fréquents (dans The New Adventures of Old Christine ou bien de façon systématique dans Cougar Town où les verres de l'hérö̈ne sont démesurés) et reliés à des situations dysfonctionnelles. Le vin rouge symbolise tout à la fois la nécessité de se relâcher, l'octroi par les femmes d'un moment à elles (qu'il soit joyeux ou triste) et la sur-responsabilisation des femmes : comme l'a bien remarqué Alain Ehrenberg, puisque la deuxième modernité a rendu la responsabilité plus prégnante que la culpabilité, le sentiment d'insuffisance est une cause majeure des formes dépressives et de la consommation de substances psychotropes.

Pour autant, ces protagonistes ne sont pas des anti-héroïnes, comme cela peut être le cas pour des personnages masculins comme le misanthrope Gregory House (Dr House), le tueur en série Dexter (Dexter) ou le cupide
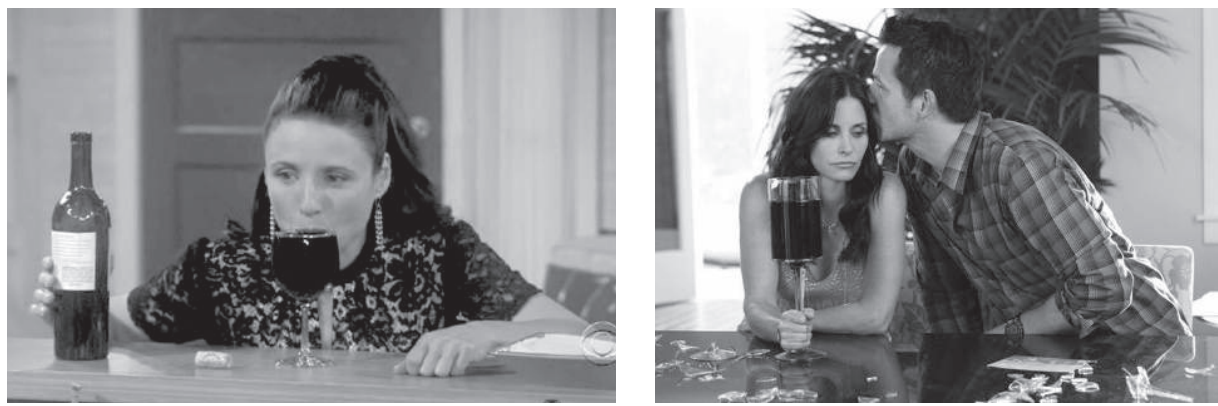
Christian Troy (Nip/Tuck). Les débordements que ces femmes vivent n'empêchent pas la persistance d'un care profond pour leur entourage. Femmes fortes précisément parce qu'elles reconnaissent leurs diverses vulnérabilités, les héroïnes vacillent certes, mais en profitent pour interroger les angles morts de la conjugalité et de la maternité. Le choc qui fait basculer le couple d'un mariage romantique à un monde post-romantique révèle donc les trop nombreuses responsabilités qui incombent aux femmes, et qu'il ne leur est plus possible de tenir. L'idéal de l'épouse parfaite promulgué par le rêve américain se craquelle devant la démultiplication des identités engendrées par l'émancipation des femmes, qui en plus d'être mères, épouses, amies, amantes et gestionnaires domestiques, doivent composer avec un travail souvent prenant. The New Adventures of Old Christine s'ouvre sur cette sur-responsabilisation des femmes. L' héroïne se réveille au milieu de la nuit pour laisser un message sur son propre répondeur, tactique schizophrène d'une mère célibataire débordée, que vient souligner son insistance à se remercier elle-même : «Salut, c'est moi. Message pour moi-même. Deux, trois choses : heu, mettre plus d'argent dans le sac de Ritchie [son fils] pour le déjeuner, acheter du lait, acheter du vin, trouver d'où vient cette odeur bizarre dans le salon. Du botox, peut-être. Je sais pas. Arrête de prendre du NyQuil, je crois que ça te tient éveillée. Ok, ouais, c'est tout. Merci beaucoup !... Tu sais quoi, non, pas de Botox. C'est dégoûtant. C'est quoi ton problème ? Apprends à t'aimer, nom de Dieu. Ok, ouais, c'est tout. Heu, bonne nuit. » La scène suivante se déroule le lendemain matin : le répondeur restitue à Christine, qui mange du Nutella à la petite cuillère, les nombreux messages qu'elle s'est laissés, listant de nouvelles tâches et conseils parmi lesquels « Arrête[r] de manger du sucre, ça ne remplace pas l'amour. » Le monologue fait référence à l'explosion des tâches, qui sont à la fois maternelles (le déjeuner de son fils, le lait), personnelles (le vin, le NyQuil), domestiques (l'odeur bizarre) et féminines (le botox, la compensation par le sucre). L'usage de la deuxième personne du singulier souligne des pans multiples de la personnalité se répondant l'un à l'autre, sous le coup des injonctions contradictoires faites aux femmes de plus de quarante ans : être divorcée mais responsable de tout, séduisante mais naturelle, alerte mais dormant sans remèdes. Parce que les récits réintègrent la dimension structurelle des insatisfactions féminines, les obligations masculines deviennent un élément clé des récits, contrastant avec l'insouciance 
qu'avaient les partenaires des hérö̈nes trentenaires. À la façon de vases communicants, la responsabilisation des hommes répond désormais à la déresponsabilisation des femmes, ce qui n'est pas sans recréer de nouvelles asymétries. L'infantilisation des époux apparaît comme un corollaire fréquent de l'empowerment des femmes par leur gestion, ou au contraire leur abandon, de la domesticité. Cependant, aucun des époux n’est satisfait de ce rapport de pouvoir. Au contraire, tous apparaissent prisonniers de rôles et d'habitudes. Pour la première fois, les personnages masculins doivent s'adapter aux mutations féministes des héroïnes. Quand le care féminin est concurrencé par l'émancipation, le partage des tâches domestiques, des charges mentales et des marques affectives devient impératif. Autonomie et responsabilité sont redistribuées aux hommes en même temps que liberté et épanouissement deviennent pour les femmes, non pas une priorité absolue, mais une variable qui n'est plus négociable. D’un côté, les personnages masculins participent de plus en plus à l'élaboration du couple, non sans difficultés, comme dans The Good Wife où Peter peine à se détacher de ses habitudes patriarcales (ne comprenant pas, par exemple, pourquoi Alicia met tant de temps à le pardonner) et où Will est surpris qu'Alicia reste insensible à ses propositions romantiques. D'un autre côté, conséquence de la responsabilisation masculine, les femmes découvrent les vertus de l'égoïsme. Dans Cougar Town, l'égocentrisme est favorablement redéfini en ce qu'il décentre l'obsession de la perfection pour privilégier l'authenticité. Après que Jules a appris que, malgré tous ses efforts, ses performances sexuelles sont décevantes, elle cesse de se préoccuper des artifices féminins et se concentre sur son seul plaisir. Le sacrifice des envies et des plaisirs, qui était tant exhorté dans les sitcoms familiales des années 1950 et 1960, devient contre-productif à l'heure où, selon les valeurs individualistes, le respect des autres passe par le respect de sa propre personne.

\section{Les ondes de choc de l'individualisme jusqu'à la maternité}

À partir de 2004, beaucoup d'hérö̈nes expriment leur maternité par un surinvestissement vis-à-vis de leur(s) enfant(s), tant et si bien que de nombreuses relations apparaissent comme abusives : certains personnages transfèrent la perte émotionnelle de leur divorce dans la maternité 
(Susan, Desperate Housewives; Cougar Town; The New Adventures of Old Christine), quand d'autres, voulant trop bien faire, étouffent leur progéniture (Bree, Desperate Housewives) ou perdent pied dans des pratiques illégales (Weeds, Nurse Jackie). À l'heure où la structure conjugale se délite, l'amour romantique semble se réfugier dans des relations que le « sang » rend, en apparence, éternelles. Toutefois, la fracture entre l'amour porté à autrui et ses modalités concrètes s'exprime aussi dans ces relations parentales. Les maternités se font plus faillibles, abandonnant des territoires jusqu' ici inviolables ou assumant des agacements autrefois cachés : dépassée par la consommation de drogues de sa fille, Jackie donne à son époux la garde des enfants ${ }^{14}$; voyant ses jumeaux devenus adultes revenir à la maison avec leurs valises, Lynette court fermer les verrous de la maison pour les empêcher d'entrer (sans succès, elle abdiquera) ${ }^{15}$; Alicia fond en larmes lorsque sa fille, qui vient d'apprendre la séparation de ses parents qu'elle doit garder secrète, l'implore de « les protéger davantage », elle et son frère ${ }^{16}$; Cathy, exaspérée des blagues de mauvais goût de son fils, se met en scène dans une baignoire pleine de sang, faisant croire à son suicide avant de réaffirmer son autorité en plaquant violemment son fils contre un $\operatorname{mur}^{17}$. Curieusement, le seul abandon maternel est représenté dans Desperate Housewives sur le network $\mathrm{ABC}$ et provient du personnage le plus conservateur, ce qui brouille un peu plus les frontières supposées entre la subversion des chaînes câblées et le conformisme des networks. Vers la fin de la deuxième saison, Bree entretient une relation de confiance avec son parrain des alcooliques anonymes, un ancien alcoolique et dépendant sexuel. Leurs rapports reposent sur une forte agapè (condition sine qua non de leur guérison), ce que Bree ne manque pas de comparer avec les mensonges de ses relations passées. Cette relation est toutefois mise en danger par l'enfant de Bree, Andrew, qui non seulement hait sa mère après qu'elle s'est montrée particulièrement réactionnaire à l'annonce de son homosexualité, mais la tient de surcroît responsable de la mort de son père (le pharmacien de la ville, obsédé par Bree, a assassiné Rex pour se rapprocher d'elle). Pour se venger, Andrew couche avec Peter tout en prenant soin que sa mère les découvre. Le lendemain, prétendument en route pour visiter une université, Bree abandonne son fils sur le bas-côté, s'excusant de ne plus l'aimer. La radicalité de la réponse de Bree apparaît proportionnelle à la douleur consécutive à la perte de sa relation, laquelle était précieuse car elle 
se distinguait des autres par le cercle vertueux d'intimité, de confiance et d'honnêteté partagée. Le machiavélisme du personnage d'Andrew et les difficultés d'alcoolisme rencontrées par Bree encouragent une sympathie à l'égard de cette dernière, que vient renforcer la perte de qualités relationnelles nouvellement valorisées, valorisées même jusqu'à être préférées à un lien maternel factice. La représentation d'un abandon fait néanmoins figure d'exception - quoique notable ! - dans un ensemble de maternités imparfaites mais aimantes. La distinction porte plutôt désormais entre amour et obligation maternelle : si l'affection portée par les femmes à leurs enfants n'est pas perturbée par les percées individualistes, les obligations, elles, le sont radicalement. Une vision d'ensemble des mères au foyer représentées dans l'histoire de la télévision étasunienne laisse voir une maternité de moins en moins arrangeante avec les prérogatives d'épanouissement personnel. Ce n'est donc pas un hasard si l'héroïne quadragénaire la plus vertueuse en la matière est la seule qui amorce un processus définitif de rupture : Alicia Florrick est une mère virtuose qui alterne autorité parentale et dialogue démocratique, et qui est toujours soucieuse de préserver ses enfants. L'éducation impeccable qu'elle leur donne vient équilibrer, voire contrarier, la subversion du divorce au féminin puis de la construction d'une relation égalitaire : les responsabilités maternelles auxquelles se tient Alicia brident sa relation pure avec Will, en même temps qu'elles rassurent sur la qualité de l'environnement familial au moment où le mariage des parents explose.

\section{Quelle communication amoureuse dans le post-romantisme?}

Le dialogue critique qu'entretiennent désormais les hérö̈nes avec le modèle romantique porte tout particulièrement sur ses énoncés lyriques. Les femmes quadragénaires exigent des témoignages concrets de l'amour car la poésie revêt désormais pour elles une signification tout autre que la valorisation du féminin héritée de l'amour courtois. L'expression poétique, voire passionnelle, des sentiments perd du terrain face aux problèmes organisationnels de la structure conjugale. Le rapport au temps est une variable clé : les héroïnes trentenaires chérissaient les énoncés romantiques d'autant plus qu'elles étaient finalement moins tournées vers le passé que les personnages 
quadragénaires. Les récits du passé des protagonistes, entre 1997 et 2004 , ne sont pas totalement absents mais ils sont rares : on ignore la façon dont Carrie Bradshaw est arrivée à New York et ce qu'elle a fait avant et l'on n'a que de courts et incomplets récits de celle dont Sydney Bristow, ou encore Buffy Summers, sont devenues espionnes et Tueuse de vampires. De son côté, Ally McBeal ne se remémore ses années universitaires que parce qu'elle y a perdu son grand amour, et Dharma et Greg se racontent peu ce qu'ils ont fait avant de se rencontrer. De plus, les discussions sur le futur sont individualistes : elles traitent de l'ouverture des possibles que permet l'émancipation féminine, sans appeler de leurs vœux une réorganisation systémique. Or, les femmes quadragénaires ramènent dans l'instant présent les problèmes dus à la construction passée du couple et veulent en refondre immédiatement l'organisation future. Cette exigence de penser les risques inhérents à la structure conjugale est due à une situation présentement instable. Dans The Good Wife, lorsque Will déclare sa flamme, Alicia est sur le point de monter sur scène pour soutenir publiquement son époux, et dans The Big C, Cathy se sait condamnée par un cancer au stade avancé. L'expérience amoureuse de ces dernières est un élément nouveau des séries télévisées : les héroïnes ont appris de leurs erreurs et savent que, pour se perpétuer, l'hégémonie masculine doit concéder quelques flatteries. Tandis que les discours romantiques des personnages masculins produisent un amour autoréférentiel, les hérö̈nes, en le ramenant aux contingences immédiates, le replacent dans les « processus sociaux plus larges »dont le romantisme voulait historiquement se défaire ${ }^{18}$. Des scènes explicitent sans détour la rationalisation des manières féminines d'aimer. Dans The Big C, alors que Cathy s'est séparée de son époux, celui-ci essaie de la reconquérir en reconstituant dans leur salon la plage sur laquelle il l'a demandée en mariage. Paul se fend d'un discours poétique qui mène l'héroïne aux larmes, mais ne la convainc pas pour autant :

PAul : - Catherine Elizabeth Tolkie [...] À l'instant où je t'ai vue dans le bureau des étudiants, à coller des affiches pour la fête du printemps, j'ai su que je t'aimais. J'ai aimé ta façon d'accrocher scrupuleusement les posters avec ton pistolet à agrafes et j'ai aimé la façon dont tu as ri quand tu as réalisé qu' ils étaient à l'envers. (Il fait une incise :) Garde à l'esprit que 
j'ai vingt-cinq ans, là. J'ai des infos plus récentes mais j'essaie de rester fidèle à ce qu'il s'est passé. (Il reprend.) J’ai aimé que sous cette carapace de grincheuse, batte le cœur d'une jeune fille qui aimait la danse et la bagatelle, que tu fis chacune très bien trois fois en une nuit si ma mémoire est bonne. Tu es le Yin de mon Yang, le Ping de mon Pong, la normalité de ma folie. Soyons nous pour toujours, à l'infini. Je veux que nous devenions de vieux idiots incontinents ensemble. Épouse-moi, Cathy. $[\ldots]$

CAтнy : - Je ne suis plus cette fille normale.

PAUL : - C'est pas grave. Moi, j'ai perdu mes abdos.

CAтну : - Mais tu es toujours ce gars, ce gars qui fout du sable dans mon salon et qui ne se demande pas qui va nettoyer. PAul : - Oh ça va, je nettoierai le bazar. Bon sang Cathy, tu ruines tout ! Tu sais qu'avant, tu adorais ce "gars" qui faisait ce genre de trucs débiles. Tu as épousé le "gars" qui fait ce genre de trucs débiles !

CAтнy : - Je sais. C'est juste que... les choses ont changé. J'ai changé. Je ne sais pas si toi tu peux changer et je ne sais pas si j'ai le droit de te le demander ${ }^{19}$. $\gg$

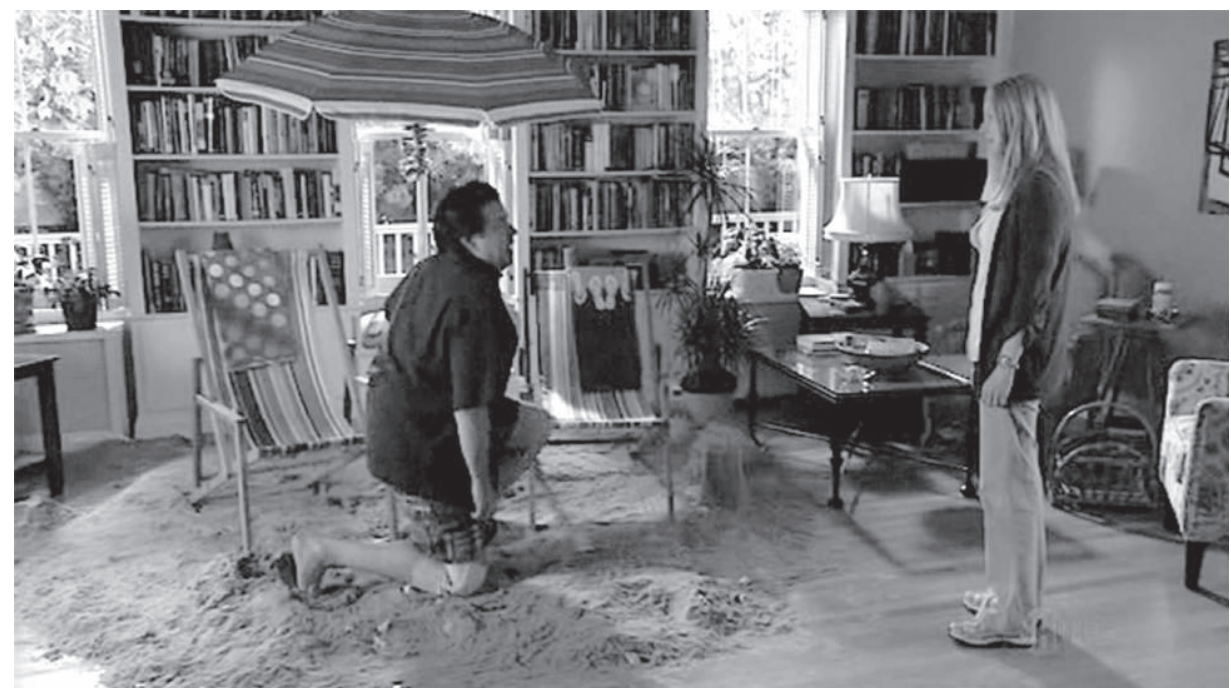


Une forte incompréhension empêche les époux de se réconcilier : alors que Paul trouve romantique de faire revivre le passé pour y retrouver les premiers instants passionnels, Cathy décode cette communication comme l'indice de ce que son époux ne comprend pas ses revendications égalitaires du temps présent, pour le temps futur. Le discours de Paul recouvre toutes les caractéristiques du romantisme : il est un élan lyrique, à la syntaxe périlleuse, qui vante la fusion des partenaires, mais Cathy n'est plus dupe des rapports de pouvoir que cache la supposée complémentarité du yin et du yang, du ping et du pong, et surtout du normal et de la folie. Elle refuse d'être l'épouse pragmatique qui nettoiera le sable répandu par la folie amoureuse de son époux. Dans The Good Wife, c'est le rappel rationnel de la conjugalité qui disqualifie la passion des débuts. Lorsque Will avoue ses sentiments par téléphone, il s'entend répondre que « La poésie, c’est facile [mais que] ce sont les réunions de parents d'élève qui sont difficiles. » La scène est restée célèbre auprès des fans de la série, certainement parce qu'elle prend à contre-pied la déclaration d'amour classique en même temps qu'elle confirme le sérieux des sentiments de l'héroïne. Alicia, qui a été blessée par le rêve romantique, n'est donc pas touchée par la passion et veut plutôt « planifier » l'amour, ce qui apparaît incongru à Will. Ce dernier acquiesce sur le moment... pour rappeler quelques instants plus tard, et reprendre de plus belle son élan lyrique. Les revendications de l'héroïne sont en grande partie ignorées, le masculin semblant démuni face à cette requête de rationalité amoureuse. Les façons genrées d'aimer sont brouillées. Ce ne sont plus les femmes qui sont avides de mots doux, et les hommes qui privilégient des preuves pratiques, mais l'inverse : la détraditionnalisation dévalorise la poésie. Désormais conscientes que « les plus beaux rêves féminins d'amour romantique se sont trop souvent terminés par les plus sordides assujettissements domestiques ${ }^{20} \gg$, les femmes préfèrent l'égalité concrète plutôt que la fusion sentimentale. En retour, la conversion des personnages masculins aux codes communicationnels romantiques montre précisément leur incompréhension des aspirations individualistes féminines, à laquelle ils ne savent répondre que par des codes traditionnels, c'est-à-dire antérieurs à l'émancipation. 


\section{Notes}

1 The New Adventures of Old Christine, saison 1, épisode 1.

2 « Happily », simultanément adverbe et adjectif, signifie ici «joyeusement », « comblée » et « aisément »: la locution fait référence à celle, bien connue, d'« happily married ».

3 Happily Divorced, saison 1, épisode 1.

4 Carter, Bill, « With “Homeland”, Showtime Makes Gains on HBO », New York Times, 29 janvier 2012. Disponible en ligne : http://www. nytimes.com/2012/01/30/business/media/with-homeland-showtime-makes-gains-on-hbo.html?pagewanted=all\&_r $=0$, consulté le 22 septembre 2014.

5 Kinon, Cristina, « "Nurse Jackie” Star Edie Falco, Mary-Louise Parker, more strong women lift Showtime », Daily News, 15 février 2009, disponible en ligne : http://www.nydailynews.com/entertainment/tv-movies/nurse-jackie-star-edie-falco-mary-louise-parkerstrong-women-lift-showtime-article-1.363757, consulté le 22 septembre 2014.

6 «It's Showtime for Compelling Female Roles », The Independent, 20 avril 2010, disponible en ligne : http:// www.independent.co.uk/ arts-entertainment/tv/features/its-showtime-for-compelling-femaleroles-1948695.html, consulté le 22 septembre 2014.

7 Interview d'Andrea Press. Voir Fallon, Kevin, « Showtime: Television for Women, for Everyone ? », The Atlantic, 16 août 2010, disponible en ligne : http://www.theatlantic.com/entertainment/archive/2010/08/ showtime-television-for-women-for-everyone/61535/, consulté le 22 septembre 2014.

8 Kinon, Cristina, ibid.

9 Kinon, Cristina, ibid.

10 Durant la dernière saison, sa détermination toute traditionnelle à protéger ses amies contre les répercussions du crime qu'elles ont commis ensemble se retourne finalement contre elle: Gabrielle, Susan et Lynette, exaspérées que Bree prenne seule des décisions qui les concernent toutes, coupent toute relation.

11 Ehrenberg, Alain, La Fatigue d'être soi. Dépression et société, Paris, Odile Jacob, 2000. 
12 Desperate Housewives, saison 1, épisode 6.

13 Desperate Housewives, saison 1, épisode 8.

14 Nurse Jackie, saison 5, épisode 10.

15 Desperate Housewives, saison 8, épisode 14.

16 The Good Wife, saison 2, épisode 21.

17 The Big $C$, saison 1, épisode 1.

18 Giddens, Anthony, La Transformation del'intimité. Sexualité, amour, et érotisme dans les sociétés modernes, Rodez, Éditions du Rouergue, 2004, p. 55.

19 The Big C, saison 1, épisode 3.

20 Giddens, Anthony, ibid., p. 81. 



\section{Entre les désillusions du romantisme et les sables mouvants du mariage}

\section{Desperate Housewives: les émotions dangereuses}

Quelques mois après la fin de Sex and the City, le network ABC commence la diffusion de Desperate Housewives, dont le genre, entre drama et soap opera, privilégie une approche simultanément stratégique et émotionnelle de récits portés sur la face cachée du rêve américain. La série repose tout d'abord sur la subversion de l'image de la parfaite femme au foyer si présente dans les séries télévisées des années 1950 et ambitionne de révéler les écarts entre les sourires d'apparence et un désespoir domestique bien réel. La banlieue américaine agit, à l'instar du « cinquième personnage » de Sex and the City qu'était New York, comme un élément fondateur du récit : le fait que, dès l'ouverture de la série, soit décrite la position des maisons et le fait que les premiers plans soient ceux d'ensemble du voisinage et de ses habitants suggèrent la description d'un quadrillage structurant. On retrouve une telle image dans le générique de Weeds, où une vue d'avion donne à voir les maisons de si haut qu'elles semblent n'être que des petits cubes alignés, soigneusement enserrés d'une route principale, tandis que la chanson du générique décrit « des petites boîtes à flanc de colline » (《little boxes on the hillside $»)$.

Desperate Housewives, tout comme The Good Wife ou les séries de Showtime, réinsère la dimension profondément structurelle de l'oppression patriarcale qui faisait défaut à bien des imaginaires médiatiques dans les années 1980 et 1990. Comme le souligne Anna Marie Bautista, «Desperate Housewives semble à la fois réaffirmer et démanteler cette idée 
que la maison est un havre de paix pour les femmes ${ }^{1}$. » Le problème des héroïnes est double : ce qui est extérieur au foyer menace par son étrangeté en même temps qu'il confère à la maison une fonction protectrice, malgré le fait qu'elle soit par ailleurs source de contraintes et d'assujettissement. Ce protectionnisme familial travaille depuis longtemps le cœur du rêve américain, ce dont témoigne le préambule de la Constitution des États-Unis de 1787 qui défend la protection de la «tranquillité domestique ». Ce n'est ainsi pas un hasard si Bree, le personnage qui s'attache le plus à préserver la représentation du rêve américain, raconte avoir rencontré son époux lors d'une convention républicaine. Le récit insiste à cet instant sur le partage de valeurs traditionnelles, télescopant les mythes romantique et nationaliste : l'amour entre Bree et Rex s'ancre dans, et ancre en retour, le rêve américain de la prospérité et de la liberté... mais c’est également lui qui vient saboter de l'intérieur, quelques années plus tard, la dynamique familiale.

Le retour dans les banlieues ne signifie donc pas mécaniquement une réassignation des personnages principaux féminins au foyer familial mais une réinterrogation des conséquences d'un tel ordre domestique. Le principe originel de Desperate Housewives, qui consiste à faire voir ce que l'architecture de la propriété privée cache, révèle les angles morts de la famille nucléaire : la froideur de Bree, la cupidité de Gabrielle, l'exaspération répétée de Lynette et le manque d'assurance de Susan sont la suite logique de l'oppression des femmes, qui vient endommager les contrats amoureux en empêchant toute démocratie relationnelle et en encourageant même la manipulation interpersonnelle. Les femmes quadragénaires subissent donc une double peine : elles doivent composer avec la mort du romantisme tout en gardant intactes les apparences du rêve américain.

Le double problème de l'obsolescence du rêve américain et du maintien de sa représentation pour autrui n'est pas l'exclusivité de Desperate Housewives mais c'est bien dans cette série que le problème s'exprime le plus. Le conflit qu'entretiennent les femmes avec la résistance de la première modernité est au cœur de la série, de son idée même jusqu'à son titre. Lorsqu'il raconte comment il a pensé à cette histoire, Marc Cherry confie qu'elle est née d'un reportage sur une mère infanticide regardé avec sa propre mère : « Je [lui] ai dit “Bon sang, tu imagines une femme si désespérée qu’elle pourrait tuer ses propres enfants ?” Ma mère a retiré sa cigarette de sa bouche, s'est tournée vers moi et m’a dit "Je suis passée par là"2. » De surcroît, le titre même de 
la série insiste sur le « désespoir » féminin, c'est-à-dire sur le manque de perspective et d'espérance des femmes du fait de leur assignation au foyer. Ainsi enfermées dans des formes conjugales de la première modernité dont elles ne sortent que peu (dans Desperate Housewives, rares sont les moments de célibat, qui sont de toute façon tristes), les récits explorent les frustrations et les dépossessions infligées aux femmes. Cette injonction de maintenir des valeurs obsolètes fait que Desperate Housewives regorge d'incompréhensions amoureuses, ce qu'entérine le genre de la série, qui révèle ce décalage entre apparences et pensées : d'un côté, le style proche du soap opera réaffirme cet écart entre ce que les femmes disent, pensent et font sous l'influence des valeurs, des normes ou des injonctions qui pèsent sur elles, de l'autre, l'esthétique « camp », comme l'a souligné Niall Richardson ${ }^{3}$, en ce qu'elle est dénuée de technologies et est saturée de couleurs vives, vise à expliciter l'obsolescence de ce monde.

Signe de leurs efforts pour s'insérer dans l'ordre traditionnel des genres, du couple et de la famille nucléaire, les héroïnes font constamment montre d'insuffisances ou d'excès mais trouvent rarement un juste équilibre. D’un côté, les manques se font ressentir dans la performance féminine : la maladresse maladive de Susan prévient toute élégance féminine et l'exaspération de Lynette quant à l'éducation de ses enfants donne à voir une maternité faillible. De l'autre, l'exagération pèse sur Bree, condamnée à la perfection, et sur Gabrielle, prisonnière de sa cupidité. Les efforts pour maintenir, non plus le rêve américain, mais sa seule représentation forment les ressorts tragi-comiques de la série. Un exemple est resté célèbre, issu du pilote de la série dans lequel le mari de Bree lui reproche sa perfection, laissant son épouse aller pleurer en silence dans la salle de bains, devenue coulisses de la représentation sociale. Bree ressort finalement sur la mélodie d'une musique doucement enjouée, prête à continuer le petit théâtre de la parfaite femme au foyer. Les efforts des hérö̈nes passent rarement par une réponse argumentée et démocratique mais par la continuation de l'ordre malgré son obsolescence tout juste reconfirmée. Il est flagrant de voir que les personnages, masculins comme féminins, sont enfermés dans des identités qu'ils ne veulent pourtant pas performer, tout particulièrement les femmes qui ont des comportements excessifs. Gaby trouve dans sa beauté le meilleur moyen d'assouvir sa soif d'argent, laquelle prend sa source dans son origine sociale modeste et les violences sexuelles subies durant son adolescence. 
Le contrat conjugal ainsi conclu entre elle et Carlos repose sur l'échange très classique de capitaux esthétiques contre financiers. Il est néanmoins ce qui les éloigne le plus puisqu'il enferme l'épouse dans une prison dorée et le mari dans une poursuite jamais satisfaite de richesse. Bree, quant à elle, incarne l'obsession de la perfection domestique, mais ses névroses se transforment en dominations qui étouffent ses enfants et émasculent son mari. De la même manière, lorsque les insuffisances de Lynette se transforment en volonté de tout contrôler, Tom la quitte, faisant exploser le couple qui était jusqu'ici le plus stable : Lynette avoue alors ne pas se sentir dévastée mais « soulagée ${ }^{4}$ ». Les formes d'exagération entraînent ainsi, tant dans le couple de Gaby que dans celui de Bree ou de Lynette, une coupure de communication : Rex et Bree ne s'écoutent plus tandis qu'à tour de rôles, Gaby et Carlos se manipulent pour atteindre leur propre épanouissement (ainsi Carlos truquant la pilule de Gaby afin qu'elle tombe enceinte malgré elle, ou Gaby ordonnant à son époux en voie de rédemption de revenir à des activités plus lucratives).

Parmi les stratégies déployées pour freiner la dangerosité des émotions, la dissimulation figure en bonne place, que mobilise le plus le personnage de Bree van de Kamp. Niall Richardson a bien identifié les politiques camp de cette héroïne : la prévalence de l'apparence et de la stylisation, articulées au genre féminin, construit un personnage dont la féminité est hyperbolique. L'exagération des performances domestique, féminine, maternelle ou encore conjugale de Bree jette un doute sur ce qu'il se cache sous ce vernis de la première modernité. Il faut attendre les derniers épisodes de la série pour obtenir une explicitation de ces stratégies. Au cours d'une double analepse, est expliquée l'origine de ce qu'elle nomme son « masque ». Dans une longue séquence, deux scènes éclairent le comportement froid et obsessionnel du personnage. La première scène montre Bree, encore enfant, lors d'une discussion avec sa mère. Celle-ci, qui vient d'apprendre que son époux la trompe, explique à sa fille qu'il faut cacher ses émotions afin de ne pas perdre de « pouvoir » (le terme est explicite) vis-à-vis des hommes, indignes de confiance. Elle lui enseigne à porter un « masque » : «C'est le visage que tu portes quand tu ne veux pas que les gens sachent ce que tu ressens. C'est très utile, tout particulièrement quand tu as affaire aux hommes [car,] si un homme sait ce que tu penses, cela lui donne du pouvoir sur toi. » Expliquant plus longuement à sa fille qu'elle a oublié comment porter son masque après 
toutes ces années de mariage, elle lui offre une deuxième solution qu'est la cuisine. À cet instant, l'amour n'apparaît en aucun cas comme une modalité relationnelle prompte au partage ou à la sécurité émotionnelle mais comme une vulnérabilité menant fatalement à la domination masculine, du fait des inégalités typiques de la première modernité.

La deuxième scène de cette séquence montre la conversion définitive de Bree à la stratégie du « masque » : après que Rex lui demande d'abandonner ce visage « plastique », référence à l'artificialité de la représentation d'une poupée domestique, Bree s'ouvre finalement et avoue son état émotionnel : elle est « blessée et humiliée » que Rex ait complimenté Gaby plus tôt dans la soirée. Toutefois, en lieu et place d'une discussion démocratique, l'héroïne reçoit pour réponse, pire qu'un silence, une moquerie de la part de son époux suivie d'une disqualification brutale de ses ressentis jugés ridicules. Repensant à sa mère, Bree se referme définitivement sur ellemême et clôt la discussion par la promesse du petit-déjeuner favori de son époux : à l'instar de la tarte préférée que sa mère cuisinait à son père pour le distraire, Bree, ne pouvant plus tenir le masque, détourne l'attention de son époux. La cuisine, qui agissait comme une métaphore de l'oppression des femmes dans I Love Lucy ou comme un exutoire dans Maude, devient dans Desperate Housewives un mécanisme de défense, une tactique de diversion dans la guerre domestique. L'obsession de Bree pour la cuisine, que l'on peut au premier abord analyser comme une névrose ménagère, se révèle donc être avant tout une protection émotionnelle, renforcée par les compétences culinaires envisagées comme outils de manipulation.

\section{Eros contre Thanathos dans The Big C: le sursaut féministe d'une mourante}

L'explosion conjointe du rêve romantique et du rêve américain apporte une souffrance qui est souvent ramenée aux rêves personnels sacrifiés. En la matière, les interactions maritales les plus incisives sont celles issues de la première saison de The Big C. L'héroïne est Cathy Jamison, une enseignante, mariée et mère d'un adolescent, qui découvre être atteinte d'un cancer de la peau au stade avancé. Les espoirs sont maigres pour cette quadragénaire et la série raconte précisément la découverte de soi, dans un combat contre la montre. Pour son interprète Laura Linney: 
«Elle apprend de son expérience, elle apprend des erreurs qu'elle fait, elle apprend de ses actions positives et, plutôt que de faire une liste des choses à faire avant de mourir, je crois qu'elle essaie de savoir qui elle veut être. Beaucoup des choses qu'elle fait sont drôles parce qu'il y a un sentiment de libération, qui est curieux : quand vous allez mourir, vous êtes libre de faire ce que vous voulez, vous vous donnez la permission de le faire ${ }^{5}$. La série commence après que Cathy a appris être malade, et après qu'elle a expulsé son époux du foyer familial, exaspérée par son immaturité et son irresponsabilité domestique. La thématique de la mort et celle, corrélée, du manque de temps, ouvrent la voie à une réactualisation des revendications de la deuxième vague féministe quant à une réorganisation de l'espace domestique, qui avaient été balayées par les réussites professionnelles et la grande indépendance des working women de la décennie précédente (Sex and the City, Ally McBeal).

En plus de ce sursaut caractéristique du féminisme de la deuxième vague contre les charges domestiques, Cathie Jamieson redécouvre son corps dont elle dit avoir eu honte, les métastases étant paradoxalement l'occasion d'une redéfinition généralisée : elle demande à son médecin d'évaluer la beauté de sa poitrine, bronze nue dans son jardin et se fait intégralement épiler pour son propre plaisir. Cette réappropriation aux dimensions néoféministes (« Avant, je pensais que les femmes qui s'épilaient étaient des antiféministes qui se haïssent, mais je comprends l'envie... ça me fait me sentir puissante ${ }^{6}$. ») s'accompagne d'une redécouverte de la sexualité. Libérée des injonctions sociales, Cathy est libérée corporellement et amorce une relation pure avec Lenny, un employé du lycée dans lequel elle enseigne, dont la classe sociale (il est peintre) et la race (il est noir) dénotent la nouvelle insouciance de l'hérö̈ne, de surcroît dans des représentations télévisuelles encore frileuses lorsqu'il s'agit de couples interraciaux. Leur aventure démarre par l'amusement qu'a Cathy lorsqu'elle expérimente les sensations apportées par ces nouvelles techniques de soi et par sa réinvention identitaire : surveillant un examen dans sa classe, elle aperçoit Lenny travaillant dans le couloir et lui montre, écartant les jambes, son sexe épilé. Sans surprise, la séquence est immédiatement suivie d'une aventure sexuelle entre les deux personnages. Avant de s'écrouler au sol, Lenny, lui disant « tu es sûre que tu veux faire ça ? Je peux pas arrêter de te regarder, tu es 
tellement belle », est présenté comme un personnage attentionné et source de la reconnaissance corporelle que recherche Cathy.

Ici aussi, la relation pure apparaît d'abord par une sexualité plastique, basée sur la tendresse. Elle se compose également d'une impulsivité inhabituelle de la part de l'héroïne et d'un care cette fois masculin, faisant de cette aventure amoureuse une parenthèse libidinale dans la vie de l'héroïne : contre Thanatos, insérée dans la pulsion d'autoconservation qu'est Eros, la libido au sens freudien est libérée comme une énergie vitale. Pour Freud, le concept est à la fois celui de « l'amour que chantent les poètes », mais aussi celui de «l'amour de soi », «l'amour filial et parental », « l'amitié », « l'amour des hommes en général » et « même l'attachement à des objets concrets et à des idées abstraites ${ }^{8} \gg$. Or, le sursaut de vie de l'héroïne est précisément ce qui la projette dans la deuxième modernité, en ce sens où, affranchie de ses peurs en même temps qu'elle domestique l'idée de mourir, Cathy accepte les risques qui vont avec sa réinvention identitaire. Le rappel à l'ordre est néanmoins des plus traditionnels : de la même façon qu'Alicia Florrick (The Good Wife) met fin à une relation qui la rend pourtant heureuse pour rendre prioritaire l'éducation de ses enfants, Cathy rompt avec Lenny après que son fils Adam a failli se blesser gravement. Alors que Lenny propose son aide, l'hérö̈ne redéfinit instantanément la relation comme une parenthèse qui doit être refermée afin que les responsabilités parentales puissent être à nouveau assumées.

Sommée, mais surtout par elle-même !, de redevenir « responsable » vis-à-vis de son fils, Cathy réinvestit dans sa relation avec son fils l'amour absolu qu'elle n'a pu obtenir avec son époux, tant et si bien que leur relation devient la plus romantique de la série. Tandis que son mari Paul est aux prises avec les contingences matérielles et financières de la maladie, et que l'amour qu'ils se portent prend ainsi les dimensions de l'agapè plus que de la passion, Cathy développe avec son fils un amour poétique. Les codes télévisuels qui construisent leurs scènes sont, à bien des égards, ceux qui étaient jusqu'ici l'exclusivité des couples : une musique aux notes douces accompagne presqu' invariablement leurs scènes où Cathy formule un amour sacrificiel, trouvant son bonheur dans celui de son fils. L'unique projet postmortem qu'elle construit est à destination de son enfant : elle rassemble dans une pièce à louer, au mur de laquelle est posée en évidence une photographie d'eux deux, l'ensemble des cadeaux pour les années à venir 


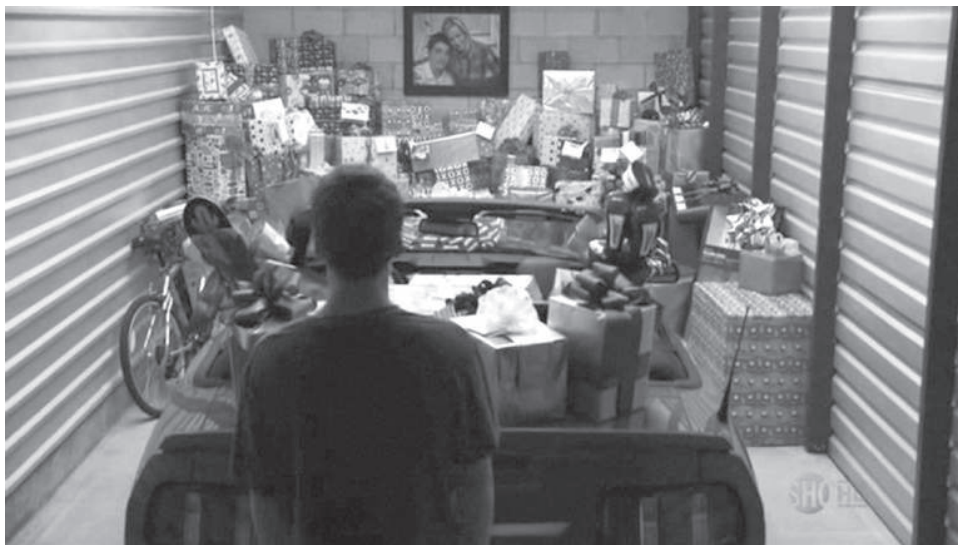

où elle ne sera plus là (anniversaires, Noëls et récompenses scolaires). Ces dons, qui ne recevront jamais de contre-dons, symbolisent la permanence du lien entre la mère et le fils au-delà de la mort en même temps qu' ils sont un transfert des envies irréalisées de Cathy sur la vie en devenir de son fils bientôt adulte. Le cadeau principal est en effet une voiture rouge décapotable, que Cathy achète d'abord pour elle ( $«$ j'ai acheté cette voiture parce que je voulais savoir ce que ça faisait [...] de se dire que tout est possible $\left.{ }^{9} \gg\right)$, avant de l'entreposer avec les autres cadeaux. C'est en les ouvrant finalement ensemble que Cathy lui demande la permission d'arrêter la chimiothérapie (laquelle n'est pas un remède mais une façon de ralentir la maladie) afin de profiter pleinement de ses derniers mois ${ }^{10}$.

En parallèle, le sursaut féministe qui marque le début de la série enjoint aux époux de réinventer leurs manières de s'aimer en même temps que la réalité de la maladie leur vole toute poésie. Après une courte séparation, les époux se réconcilient et l'agapè devient alors prédominant. L'ensemble des représentations de la série montre un couple qui peine à se retrouver (ils communiquent difficilement et s'éloignent régulièrement), du fait non seulement des expériences très divergentes qu' ils vivent, mais surtout du rapport inégal qu'ils ont au temps. Cela s'exprimera jusque dans la fin qui leur est donnée : une clôture est offerte à la relation entre la mère et l'enfant qui n'est pas accordée au couple conjugal. D'un côté, l'adolescent autrefois difficile surprend Cathy en obtenant ses diplômes un an plus tôt, réalisant le rêve de sa mère de le voir finir son parcours lycéen. De l'autre, 
Paul décide d'acheter un bouquet de pivoines à son épouse mais la gerbe de fleurs devient funéraire lorsqu'entrant dans le salon pour les lui offrir, il découvre qu'elle est morte. Les créatrices expliquent ainsi cette scène : « il y a cet époux, qui n'a pas vraiment été un bon époux durant la série, qui arrive enfin, et c'est le moment où elle trépasse. Beaucoup de personnes qui ont le choix de décider de l'instant où ils meurent, même si c'est subliminal, décident de le faire quand tous ceux qu'ils aiment ont quitté la maison ou la pièce. Nous voulions jouer avec cette idée qu'elle était seule quand elle est morte, et qu'elle a fait ce choix. ${ }^{11} \gg$ En même temps que la série s'attache à représenter le caractère imprévisible de la mort, il est intéressant que son époux soit celui qui en pâtisse, en ce sens où il n'a pas eu l'occasion de formuler des adieux formels ni de prouver concrètement son amour. L'agapè, qui était devenu leur régime d'amour, ne peut trouver une conclusion que dans son obsolescence : sa qualité première étant le compagnonnage bienveillant, attaché au bien-être de l'autre (ici majoritairement physique et physiologique), il trouve logiquement sa fin dans la mort de l'héroïne.

\section{Néoféministes à quarante ans}

D'autres médiacultures articulent le passage à la quarantaine avec l'obsolescence du néoféminisme, formulant l'une des premières critiques, ou du moins l'un des premiers bilans, de ce courant. Jules Cobb (Cougar Town) et Christine Campbell (The New Adventures of Old Christine) sont des femmes quadragénaires issues de la petite banlieue américaine (symbolisée presque littéralement dans Cougar Town où la maison de Jules est située dans ce qu'elle nomme elle-même un « cul-de-sac »). Toutes deux sont très préoccupées par la vieillesse et ses corollaires que seraient la dégradation de l'apparence physique et la difficulté de trouver un nouveau partenaire amoureux. L'ethos très féminin de ces hérö̈nes laisse deviner que Jules et Christine ont eu une beauté classique qu'elles travaillent ardemment à préserver. Toutefois, cette performance de genre n'est pas sans ironie et la difficulté qu'ont ces femmes à performer le genre féminin une fois passée la quarantaine est précisément l'un des ressorts comiques de ces séries. Presqu'invariablement, le genre féminin, dont la performance devient de plus en plus difficile avec l'âge, y est posé comme un problème. L'originalité de ces représentations, outre qu'elles déconstruisent ainsi le 
genre en désignant comme absurdes les canons auxquels les femmes doivent se tenir, est que les héroïnes apparaissent tiraillées entre le fait de fournir les efforts demandés sans toujours y parvenir et de cesser enfin de taire à quel point il est difficile de performer ce genre. Discutant ainsi avec une amie vingtenaire, Jules Cobb lui explique que : «On a quarante ans : pour nous, se préparer pour coucher avec quelqu'un, c'est comme se préparer pour une mission spatiale. » La séquence qui suit montre les préparatifs en question, rendus comiques par un traitement presque olympique : ordonnant à son esthéticienne de l'épiler en même temps que sa collègue lui fait une manucure, celle-ci répond mi-admirative, mi-incrédule, « Personne n’a

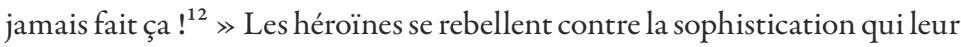
est demandée mais continuent de s'y plier car elles ne voient pas d'autres échappatoires à leur solitude de divorcée. Ce statut, doublé de l'amitié qu'elles ont gardée avec leur époux, les amènent à comparer les attendus sociaux qui pèsent sur elles avec ceux qui pèsent sur eux : dans The New Adventures of Old Christine, Christine est confrontée, dès le pilote, au fait que Richard a une nouvelle petite-amie... nommée Christine. L'analogie de leurs prénoms amène rapidement leur entourage à surnommer l'une « la jeune Christine » ou la « nouvelle Christine » (Young Christine ou New Christine), et l'autre « la vieille Christine » (Old Christine), tendant à l'héroïne le miroir de ce qu'elle n'est plus.

La performance de genre n'est pas seulement physique : ces héroïnes sont parmi les plus névrosées et semblent pour cela être dans la continuité d'Ally McBeal. Jules Cobb est si émotionnellement dépendante qu'elle est incapable de passer une journée seule, elle fait preuve de marques d'affection démesurées (son petit-ami lui fait remarquer qu'elle semble vouloir « poignarder les gens avec son os pelvien ${ }^{13}$ » lorsqu'elle les enlace) et elle sollicite sans cesse des preuves d'amour. De son côté, Christine Campbell, rare anti-héroïne, est si égocentrée qu'elle peine à se réjouir du bonheur d'autrui, bonheur qu'elle envisage toujours comme une concurrence ou, là encore, comme un miroir tendu à ses propres échecs. L'attention continuelle à soi devient dans ces formes extrêmes un égocentrisme tel qu'il entraîne une coupure communicationnelle : il est rare qu'un épisode de The New Adventures of Old Christine ne montre pas l'héroïne se moquant éperdument de ce que lui disent ses proches et ramenant la conversation sur ses propres problèmes. De la même manière, le care maladif de Jules apparaît 
souvent comme une manière pour l'hérö̈ne de recentrer les problèmes d'autrui sur elle, via sa capacité à consoler ou à conseiller. Dans un épisode intitulé « Cry To Me », elle insiste pour que son petit-ami se confie à elle au sujet du décès de son père : «Voilà tes mouchoirs et ton vin [...] et tu vas t'envelopper dans cette couverture moelleuse parce qu'on veut que tu aies l'air tout petit et vulnérable ${ }^{14}$. » Lorsqu'il s'ouvre finalement à elle, elle se réjouit de le voir pleurer. La satisfaction que tirent les héroïnes du malheur de leurs proches provient de la jouissance qu'elles ont à prendre soin d'autrui, mais aussi de leur crainte d'être les seules à vivre des moments de désespoir. Cependant, le fait que les vies de ces héroïnes ne soient tout simplement pas enviables encourage à lire ces séries non comme une valorisation de l'idéologie néoféministe mais comme une représentation de ses impasses. Ces femmes sont prisonnières d'une double obsolescence : celle du genre féminin, qui devient mascarade, et celle des valeurs néoféministes, qui agissent comme la volonté répétée de « contrôler » les hommes, privilège que leur a retiré la précarisation de la vie amoureuse. Ces névroses se répètent d'autant plus qu'elles ne trouvent plus d'autres prises qu'en ellesmêmes : comme l'a bien éclairé Anthony Giddens, la tradition, qui n’a plus de légitimité, se perd dans le rabâchage ${ }^{15}$. Or, c'est là précisément le ressort comique de ces séries : le téléspectateur est invité à rire de ces héroïnes à leurs dépens. Jules Cobb et Christine Campbell sont constamment tournées en ridicule, du fait de leurs efforts pour préserver leur jeunesse et pour garder les privilèges que les femmes arrachaient aux amours traditionnelles.

\section{Nurse Jackie ou l'androgynie des manières d'aimer}

Dans Nurse Jackie, le personnage de Jackie Peyton est saturé de paradoxes et de contradictions : c'est une infirmière dépendante aux analgésiques, dévouée mais qui garde ses distances, douce mais violente faute de réciprocité. Dans la sphère privée, elle est mariée à un homme dont la masculinité est l'une des plus douces données à voir par la télévision américaine. Jackie étant en effet absorbée par son travail, c'est Kevin, personnage attentionné, qui endosse les fonctions féminines du travail domestique, notamment éducatives, proposant parmi les premières représentations d'un retournement genré des tâches domestiques. Ce renversement est d'autant plus marqué 
que Jackie sépare strictement sphère privée et sphère publique : sitôt passée la porte de son lieu de travail, elle retire son alliance, tait l'existence de ses enfants, et entretient une relation sexuelle avec le pharmacien, Eddie.

Le care de l'héroïne est simultanément l'un des plus développés historiquement de la fiction étasunienne, lorsqu'il concerne ses patients, et le plus instrumentalisé lorsqu' il s'agit de sa famille. Les récits s'attachent ici à montrer les conséquences de l'effond rement du système de santé américain sur les vies des professionnel.le.s de santé : Liz Brixius, la co-créatrice de la série, souligne que l'hôpital All Saints n’est pas rendu « glamour [à la manière de] ces autres séries qui ont un casting somptueux et des docteurs hyper intelligents qui surmontent de grands obstacles et des maladies exotiques $^{16} \gg$. Cela a pour conséquence d'apporter au care traditionnellement féminin une forte androgynie : l'hérö̈ne est certes bienveillante mais ses conditions de travail l'amènent à ne pas se laisser imposer les règles abstraites d'autrui, donc à développer de fortes capacités d'agir. Elle fait ainsi sa propre loi, pratiquant l'euthanasie, imitant les signatures des morts pour en faire des donneurs d'organe ou procurant des médicaments illégaux à ses patients, qu'elle console, rassure et appelle par des surnoms affectueux (« honey », « sweetie »). Cette éthique hors-la-loi est légitimée par son expérience qui la rend bien souvent plus compétente que les médecins. L'innovation qu'apportent ces représentations d'un care masculinisé est que l'héroïne ne s'y laisse pas déposséder : sa gentillesse disparaît sitôt que

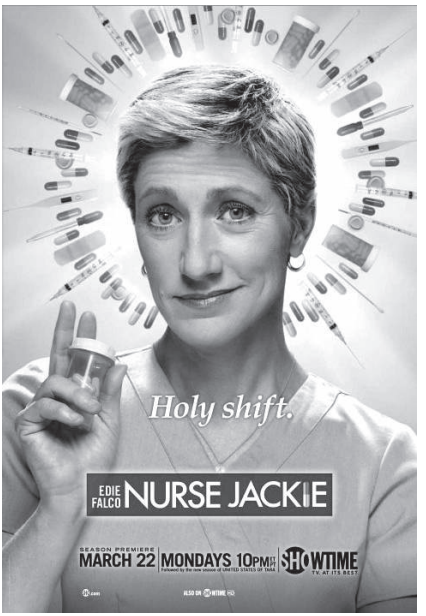

l'interlocuteur se révèle malveillant, laissant alors place à des vengeances. Dès l'épisode pilote, Jackie soigne un criminel qui a lacéré une prostituée, laquelle s'est vengée en lui arrachant une oreille. Devant l'absence de remords du patient, et apprenant qu'il ne sera pas poursuivi en justice car il dispose d'une immunité diplomatique, Jackie subtilise son oreille coupée, lui murmure « va te faire foutre » et la jette dans la cuvette des toilettes. Au-delà de son travail, l'héroïne apparaît donc dévouée au sentiment de bienveillance (c'est une « sainte », comme la désignent ses collègues, ce que viennent réaffirmer les photos promotionnelles de la série représentant son visage surmonté d'une auréole de médicaments, ou les nombreuses références religieuses de la série), tout en punissant, dénonçant, insultant les individus 
qui ne le sont pas (c'est une «vigilante », de l'aveu des créatrices ${ }^{17}$ ). Ce comportement androgyne perce dans la sphère privée où la raideur de Jackie apparaît comme contrepoint de la douceur de son époux : lorsqu'une pré-adolescente malmène sa fille, elle ne discute pas ni ne la dispute mais lui demande de « fermer sa putain de gueule ${ }^{18} \gg$. Jackie est brutalement protectrice. Ce comportement au genre trouble va de pair avec l'androgynie physique du personnage (Jackie a les cheveux courts, porte un uniforme unisexe qu'elle quitte peu, a un pas déterminé, une kinésie précise et une proxémique retenue) et avec son appartenance à la petite classe moyenne, deux caractéristiques sous-représentées à la télévision étasunienne. Elle annonce, dès l'épisode pilote, à une apprentie infirmière qu'elle «n'est pas du genre à papoter ${ }^{19}$ » et lui demandera régulièrement au cours de la série de prendre ses distances ou de se taire. Pour Liz Brixius « C'est une femme très col-bleu, qui n'est pas aux responsabilités mais qui assume les responsabilités. Elle est très humaine et très fiable - elle n'a pas une belle chevelure, elle n'est pas très maquillée, elle n'est pas plus riche, plus diplômée ou plus jolie que les personnes qui l'entourent ${ }^{20}$. $\gg$ Si sa dépendance aux médicaments l'empêche ainsi d'être une héroïne au sens fort du terme (au-delà de sa normalité, ses mensonges à des personnages attachants comme Kevin la rendent peu sympathique), ses compétences émotionnelles et médicales interdisent qu'elle soit une anti-héroïne.

L’originalité est également que les compétences émotionnelles tant représentées à compter de 2004 sont ici mises au service de la dépendance de l'héroïne aux analgésiques et, in fine, de l'anti-démocratie conjugale. Comme beaucoup d'autres héroïnes, Jackie Peyton peine à mener de front toutes ces identités et les responsabilités corrélées : la souffrance dont elle est témoin au travail et qu'elle ne peut pas toujours soulager lui est difficile, et est présentée comme l'origine de sa dépendance aux médicaments. Celle-ci est entérinée par l'incompréhension de son mari quant à son métier et par les reproches que lui formule sa fille de ne pas être suffisamment présente. Pour s'endurcir, Jackie gobe discrètement des gélules, broie et sniffe des comprimés dans les toilettes de l'hôpital, dissimule des sachets dans les interrupteurs de sa maison... mais sait refuser poliment lorsque son amant pharmacien lui propose un cachet, afin de ne pas éveiller les soupçons. À partir de 2004, les séries représentent en effet l'ingéniosité des personnes dépendantes (Desperate Housewives montre par exemple la façon dont 
Bree van de Kamp, alcoolique, cache astucieusement ses bouteilles de vin), mais l'héroïne de Nurse Jackie a la particularité de se réapproprier et de radicaliser ses compétences émotionnelles pour mentir, cacher et manipuler les gens qui l'entourent, qu'ils soient des rencontres ponctuelles ou ses proches les plus intimes. Finalement, l'empathie et l'attention que l' héroïne a développées du fait de son statut de mère et d'infirmière ont toujours pour visée le bien-être d'autrui mais, parce que l'injonction du care a mené Jackie à la dépendance, ces qualités servent un deuxième objectif qu'est la satisfaction de l'addiction. Cette ambivalence est assumée par l'héroïne qui s'amuse d'être en équilibre entre la vie et la mort, entre la dépendance et l'indépendance, entre le bien et le mal : « Si j'étais une sainte... ce que je veux peut-être, mais peut-être pas... je serais comme Auguste : il savait qu' il y avait du bon en lui, il savait qu'il y avait du moins bon, et il n'allait pas abandonner ses plaisirs terrestres avant d'être fin prêt... "Rends-moi bon, Seigneur... mais pas tout de suite"21. » Dans chacun de ses couples, avec Eddie ou avec Kevin, l'héroïne est anti-démocratique et, lorsque ses mensonges sont dévoilés, les réactions de ses partenaires amoureux dénotent leur retard émotionnel : Eddie est admiratif ( $\ll$ Tu es une menteuse exceptionnelle, putain. Un génie de classe mondiale $\left.!^{22} \gg\right)$, et Kevin, apprenant que Jackie l'a manipulé au sujet d'une décision budgétaire, est dépassé :

Kevin : - Tu as toujours su que tu allais accepter [cet argent] ? Réfléchis.

JACKIE :-Oh...J'ai fait semblant de te consulter. Mais tu le savais, bébé. Tu le sais toujours. Je savais que tu le savais.

KEVIN : - Ça ne m'avait même pas traversé l'esprit ${ }^{23}$.

Tandis que son épouse manie plusieurs niveaux ( $\ll$ je savais que tu le savais »), lui est pris au piège d'une naïveté démocratique, imaginant que les décisions sont prises dans la discussion. L'agir communicationnel de Kevin, qui souhaite parvenir à un compromis, est dépassé par l'agir stratégique de Jackie, qui cherche à influencer son époux pour parvenir à ses fins. Si elle fait preuve, auprès de ses patients, d'un care masculinisé, elle performe, auprès de ses amours, ce qui est présenté comme un patriarcat féminisé : elle formule peu ses sentiments et est fuyante mais dicte en retour les conditions de vie conjugale, soit par l'autorité, soit par la manipulation. 
Cela conduit à un renversement inédit dans les séries télévisées : le mari de Jackie se plaint régulièrement d'insatisfactions jusqu'ici réservées aux personnages féminins (sentiment d'être délaissé, colère de n'être jamais consulté, reproches quant à l'absence et au manque d'implication de son épouse, etc.) tandis que Jackie abandonne à de nombreuses reprises le territoire domestique. Une telle inversion donne aux dysfonctionnements que rencontrent les époux une cause systémique et non genrée, tout en semblant rendre impossible une redistribution efficace des tâches domestiques.

Dans un cercle vicieux, la dépendance de Jackie, qui provient de ses difficultés à remplir les fonctions qui s'imposent à elle, l'amène paradoxalement à rendre son monde encore plus fonctionnel. L'héroïne a un rapport instrumental aux individus afin de préserver son secret et de pouvoir se procurer ses drogues, de sorte que l'amour ne triomphe plus. À partir de la saison 5, la relation amoureuse qu'entretient Jackie avec Frank, un officier de police, place pour la première fois l'honnêteté comme valeur clé de leur relation, ce qui a pour conséquence inédite de rendre acceptables les défauts et les mauvaises actions d'autrui. La relation se compose ainsi d'un écroulement des fonctions et des grandes dichotomies qui structuraient les précédentes relations : du fait de leur travail respectif, Jackie et Frank sont amenés à se côtoyer à l'hôpital, rendant la distinction public/privé plus floue ; les frontières entre le bien et le mal se confondent également, replaçant non pas l'éthique mais la confiance au cœur de la relation ; enfin, la représentation de soi n'est plus subordonnée à la fonction mais s'ouvre à un expressivisme salvateur qui définit les disputes, non comme des moments d'échec, mais comme des opportunités de se connaître («Tu sais que je peux être en colère contre toi et toujours t'aimer, hein $\left.~^{24} \gg\right)$. Toutefois, cette relation idéale dans laquelle l'héroïne est visiblement heureuse ne résout pas ses problèmes d'addiction pour autant : le jour de sa première année de sobriété et après avoir passé un moment de bonheur au lit avec Frank, Jackie gobe sans raison apparente une pilule qu'elle gardait de côté, dans la boîte contenant l'alliance de son mariage raté. La dépendance de Jackie n'est explicitement pas soluble dans l'amour. Pire, la solidité de sa relation devient un argument pour étayer ses mensonges. Lors de la saison 6, lorsque Frank la surprend en train de prendre des pilules, elle lui affirme que ce n'était qu'un faux pas alors qu'elle continue en réalité de se droguer régulièrement : «Bon, tu sais ce que j'aurais fait, avant ?J'aurais menti. Chéri, j’ai 
un parrain [aux alcooliques anonymes]. Je vais aux réunions. Je vais bien et je vais continuer à aller bien, parce que j'ai trop à perdre ${ }^{25}$. » Ainsi, si de nombreux marqueurs romantiques parsèment sa relation avec Frank (bougies, moments au lit, communication intuitive), l'héroïne ne trouve en aucun cas en lui la moitié qui lui manquerait et qui résoudrait ainsi tous ses problèmes : l'amour n'apparaît pas comme un antidote à des problèmes individuels et structurels. Il est, de la sorte, profondément dé-mythologisé.

\section{Notes}

1 Bautista, Anna Marie, « Desperation and Domesticity: Reconfiguring the "Happy Housewife" in Desperate Housewives », in McCabe, Janet, Akass, Kim, Reading Desperate Housewives: Beyond the White Picket Fence, Londres, IB Tauris, 2006, p. 158.

2 Pierce, Scott D., « TV: "Desperate Housewives” Creator Owes It all to Mom », The Salt Lake Tribune, 10 mai 2012.

3 Richardson, Niall, «As Kamp as Bree. The Politics of Camp Reconsidered by Desperate Housewives », Feminist Media Studies, 6, 2006, p. 157-174.

4 Desperate Housewives, saison 7, épisode 23.

5 Bremner, Jade, « Edgy Comedy About Dealing With Cancer Comes to Screens in Dubai », Time Out Dubai, 22 mars 2011.

6 The Big $C$, saison 1, épisode 5.

7 Le terme « race » n'est évidemment pas à entendre comme une donnée biologique mais comme un produit de discours sociaux aux effets discriminants.

8 Freud, Sigmund, Essais depsychanalyse, Lausanne, Payot, 2004, p. 134.

9 The Big $C$, saison 1, épisode 4.

10 The Big $C$, saison 4, épisode 1.

11 Goldberg, Lesley, « "The Big C” Postmortem: Cathy Was "Lucky and Unlucky at the Same Time” », Hollywood Reporter, 20 mai 2013.

12 Cougar Town, saison 1, épisode 3.

13 Cougar Town, saison 2, épisode 9. 
14 Cougar Town, saison 2, épisode 14.

15 Giddens, Anthony, La Constitution de la société. Éléments de la théorie de la structuration, Paris, Presses Universitaires de France, 2012.

16 Callaghan, Dylan, «Jackie, Blue », Writer's Guild of America. Disponible en ligne : http://www.wga.org/content/default. aspx?id=3674, consulté le 22 septembre 2014.

17 Halterman, Jim, « Interview: "Nurse Jackie” Co-Creator Liz Brixius », The Futon Critic, 12 avril 2010. Disponible en ligne : http:// www.thefutoncritic.com/interviews/2010/04/12/interview-nursejackie-co-creator-liz-brixius-35266/20100412_nursejackie/, consulté le 22 septembre 2014.

18 Nurse Jackie, saison 2, épisode 6.

19 Nurse Jackie, saison 1, épisode 1.

20 Callaghan, Dylan, ibid.

21 Nurse Jackie, saison 1, épisode 1.

22 Nurse Jackie, saison 3, épisode 2.

23 Nurse Jackie, saison 2, épisode 12.

24 Nurse Jackie, saison 5, épisode 10.

25 Nurse Jackie, saison 6, épisode 5. 



\section{La rupture The Good Wife: leçons de la première modernité, jeunesse de la modernité avancée et métamorphoses de l'amour}

Preuve que les subversions n'apparaissent pas toujours là où on les attend, une rupture majeure est apparue dans les représentations télévisuelles avec une série au titre en apparence très conservateur. The Good Wife, diffusée sur le network CBS, met en scène une femme au foyer des plus classiques, confrontée aux scandales financiers et surtout sexuels de son époux politicien et, par là même, à une humiliation publique à laquelle son activité jusqu'ici circonscrite à la sphère privée ne l'avait pas préparée. Voyant son mari finalement emprisonné, elle doit subvenir aux besoins du foyer et souhaite retourner pour cela à son activité d'avocate, abandonnée treize ans plus tôt pour élever ses enfants. Mais, désormais paria, elle peine à trouver un emploi et seul son ami et amour de jeunesse Will Gardner consent à l'embaucher dans son cabinet. Épouse et mère bourgeoise, initialement parée de tailleurs étriqués et de colliers de perles qu'elle remplace par des costumes professionnels sitôt qu'elle reconquiert la sphère publique, Alicia Florrick fait donc partie de cette vague d'héroïnes quadragénaires propulsées malgré elles dans une émancipation qu'elles n'ont pas demandée mais dont, après avoir domestiqué les libertés et les possibilités, elles ne peuvent rapidement plus se passer. L'attrait et l'intérêt de la série reposent paradoxalement dans les difficultés qu'a l'héroïne à s'arracher à la première modernité, incarnée par un époux patriarcal et par des morales pesantes, et à appréhender les codes individualistes de la deuxième modernité. Dans le même temps, la trahison que représentent tant les infidélités de son époux que leur publicisation (à rebours de la promesse d'une frontière fonctionnelle entre la politique et la domesticité) et les ouvertures permises par l'épanouissement 
individuel servent de moteurs à une critique extraordinairement précise des politiques amoureuses. Tout ce complexe est sous-tendu par un rejet de la victimisation au profit d'un redéploiement des compétences personnelles, tant professionnelles qu'émotionnelles. Ce n'est pas un état de fait patriarcal ou une bonne volonté féministe qui est ici représenté, mais bien un processus post-patriarcal complexe et dynamique, fait d'émancipations et de réassignations externes comme internes à l'héroïne.

\section{La fin du romantisme et les résistances du mariage bourgeois}

Avant d'enclencher un long et difficile processus d'émancipation, l'épouse blessée décide, lors des premières saisons, de soutenir publiquement son mari, à la manière des nombreuses épouses trompées que la sphère politique nous a donné à voir ces dernières années : de Bill Clinton à Elliot Spitzer en passant plus récemment par Dominique Strauss-Kahn (auquel un épisode de The Good Wife fait référence, articulé autour d'une femme de ménage violée qui n'ose porter plainte à cause de l'influence politique de son agresseur), ces scandales politico-sexuels ont, de leur propre aveu, inspiré les créateurs. Intrigués par ce que pensaient réellement ces épouses stoïques mais blêmes, Michelle King et Robert King ont eu l'ambition d'explorer le débordement de la politique, qui ne se limite pas à la sphère de la politique professionnelle, pour en comprendre les enjeux dans le domaine de l'amour, du bureau, de la famille ou encore du couple. De telles ramifications et entrelacs ne confondent pas la sphère publique avec la sphère privée mais en montrent, caractéristique typique de la deuxième modernité, la porosité conjoncturelle ainsi que la vulnérabilité des femmes à cet égard. Alicia Florrick apparaît comme plus dotée en compétences émotionnelles, du fait de son assignation à la sphère privée ainsi qu'aux charges éducatives et relationnelles qui lui incombaient, mais moins préparée que son époux à affronter le registre public des actions, et en particulier de conséquences d'actions qui ne sont pas les siennes.

L'un des grands moteurs de l'émancipation d'Alicia Florrick, et l'un des grands moteurs narratifs de la série donc, est l'enchevêtrement du personnel et du politique, créant des agencements de pouvoir propices à l'exploration d'identités nouvelles, et allant à rebours des promesses du 
mariage bourgeois qui garantissait une imperméabilité entre privé et public. Déçue, trahie même, par ce modèle amoureux, et dans l'incapacité évidemment comme elle le dit elle-même de faire en sorte que « tout revienne à la normale », Alicia Florrick est confrontée à une impérative réinvention des politiques amoureuses. La surprise, et finalement la force innovante de la série, tient finalement à ce que cette héroïne provient précisément d'un milieu plus conservateur que les autres : pour s'extraire de l'organisation patriarcale qui a jusqu'ici structuré son existence, Alicia Florrick produit au fil des saisons, non sans peine ni backlash, une critique des rapports de pouvoir qu'elle a jusqu'ici connus. Le récit de cette série n'est pas tant celui, à la façon de Sex and the City ou d'Ally McBeal, des affres de la réflexivité et des bonheurs de la liberté individuelle, mais du long chemin qui est à parcourir pour s'affranchir du patriarcat. La solution ne consiste pas à produire une rupture définitive ou à ignorer le passé mais à faire proliférer les compétences, les capacités d'agir, les sphères et les identités : l'héroïne tente, parfois en échouant mais jamais sans en tirer les leçons, de ne renoncer à aucune de ses activités, pour faire tenir ensemble celles de mère, d'épouse, d'avocate et d'amante, tout en dépassant la trahison privée et l'humiliation publique. Cet agencement éminemment instable mais explosif amène, à la télévision étasunienne, la représentation de la première rupture au féminin.

Dans l'histoire des séries, et malgré de réelles avancées dans les politiques de représentation des femmes, les ruptures restent en effet subies par les personnages féminins. Longtemps, elles se sont produites à l'initiative des hommes (Buffy, Alias, Gilmore Girls, Ally McBeal...) ou, à minima, d'un commun accord (Murphy Brown), mais jamais véritablement à l'initiative seule des femmes. De surcroît, les représentations remettaient rarement en cause le modèle romantique : dans Sex and the City comme dans Ally McBeal, le romantisme était sauf en ce que les héroïnes concluaient que, si la relation avait échoué, c'est que le partenaire ne devait tout simplement pas être « le bon », autrement dit l'âme sœur. Dans The Good Wife, difficile de trouver des excuses à un partenaire dont les conversations sexuelles avec des prostituées ont été rendues publiques. Les rapports de pouvoir constitutifs de l'amour sont rendus violemment explicites en même temps que la structure et les identités qu'il produit deviennent la lutte centrale de la relation intime. Cette rupture au féminin permet donc de représenter (enfin !) un monde post-romantique. Bien sûr, syncrétisme médiatique 
oblige, la représentation d'une rupture par une femme a un coût : le mariage en question est exagérément bourgeois en ce qu'Alicia Florrick est dotée d'une capacité sacrificielle et d'une morale comme les séries n'en avaient pas représentées depuis les femmes au foyer des années 1950 et 1960. La vertu d'Alicia Florrick et son attachement moral au mariage (malgré les fréquentes humiliations et déceptions, l'héroïne refuse de divorcer) viennent donc adoucir la violence de la rupture, mais crée tout de même, pour l'hérö̈ne, une tension entre son oblativité, dont tout le monde profite abusivement, et sa volonté de s'en émanciper, qui est construite comme légitime mais dangereuse. Il faut également souligner que l'ethos bourgeois d'Alicia Florrick induit une communication majoritairement non-verbale, assumée par les créateurs : « Nous n’aimons pas écrire beaucoup de dialogues, parce que nous ne pensons pas que c'est la façon dont les gens s'expriment vraiment... Ils ne vous disent pas toujours ce qu'ils pensent ${ }^{1}$. » La retenue presque victorienne de l'héroïne contraste avec l'expressivisme caractéristique de la décennie précédente, des soliloques introspectifs de Carrie Bradshaw et Ally McBeal aux tirades de Lorelai Gilmore, en passant par la démocratie-fleuve de Dharma et Greg. Elle apparaît également comme une stratégie contre-hégémonique féministe répandue dans les séries des années 2000. Alicia Florrick n'est pas la seule à avoir la parole mesurée : de Jackie (Nurse Jackie) à Cathy (The Big C), les héroïnes sont également peu démonstratives, jusqu'à Bree van de Kamp (Desperate Housewives) dont le visage de cire se révèle être un masque protecteur. Cette économie de discours traduit la montée de nouveaux modes communicationnels, plus stratégiques, chez des personnages féminins qui ne sont plus dupes des pièges patriarcaux que cache la transparence romantique.

Dans The Good Wife, le chemin de l'émancipation, qui se produit sur plusieurs saisons, s'articule donc autour de la pluralisation des sphères d'activité, mais aussi de la sexualité comme territoire d'épanouissement et de l'enclenchement de relations pures qui ne dépendent pas d'un partenaire en particulier mais d'un projet individuel. 


\section{Mariage en danger et dangers du mariage}

L'émancipation de cette héroïne réside non pas tant dans l'affranchissement de son identité originelle de « bonne épouse » et de «bonne mère » que dans l'interrogation de ces identités et leur mise en concurrence avec celles d'avocate. À la façon des working women des années 1970 du Mary Tyler Moore Show ou de Murphy Brown, le féminisme libéral agit en premier lieu comme l'opérateur clé : c'est par la sphère professionnelle qu'Alicia Florrick amorce son émancipation. Mais au fil des saisons, l'hérö̈ne stabilise ses identités et affirme ses choix individuels et, à mesure que les risques s'amoindrissent ainsi, elle exprime de plus en plus sa « fatigue d'être soi », corrélaire de ces identités labiles. Pour pallier cela, Alicia recourt à des échanges de compétences entre ses identités. D’un côté, elle emprunte à la sphère privée les capitaux sociaux de son époux (lequel n'aurait sans doute pas pu les obtenir sans la réputation si positive que lui apportait Alicia : comme le souligne le président du Comité Démocratique « Sans elle, [Peter] est juste un type lambda qui a surpayé une prostituée. Avec elle, c'est Kennedy $\left.!^{2} \gg\right)$ D’un autre côté, elle transfère des compétences du public vers le privé. Ce n'est en effet pas un hasard si l'univers professionnel de cette héroïne est celui du droit. Comme dans Ally McBeal à laquelle The Good Wife fait progressivement de plus en plus de clins d'œil (via par exemple de farfelus magistrats), le monde juridique renvoie l'héroïne aux questions que lui pose son identité de femme libérée. Une collision se produit plus précisément entre une éthique bourgeoise particulièrement rigide et un espace judiciaire ouvert aux tractations et aux réversibilités interprétatives. Alicia Florrick y apprend que le monde n'est pas noir ou blanc mais composé de très nombreuses nuances qui dépendent des situations et des intérêts de chacun, de sorte qu'elle y puise des compétences pour renégocier son contrat conjugal : lorsqu'il est question que Peter sorte de prison et rentre à la maison, elle stipule immédiatement des règles fermes. The Good Wife représente donc un investissement de la sphère publique somme toute classique dans l'histoire des séries télévisées, mais parvient à boucler la boucle en faisant ensuite revenir l'héroïne vers la sphère privée, dotée de nouvelles aptitudes communicationnelles (en l'occurrence juridiques). 
C'est bien cet assemblage de deux identités, épouse politique et avocate ambitieuse, qui produit un contexte post-traditionnel : la première modernité continue de peser, à travers des codes comportementaux que l' héroïne ne peut abandonner qu'en en expérimentant d'autres que sont l'épanouissement personnel, le projet réflexif du soi et le plaisir charnel. Alicia Florrick réalise finalement que son comportement bourgeois, fait de bienséance et d'agapè, ne relève pas simplement du sacrifice, mais se retourne activement contre elle, et c'est probablement là la plus grande résistance que rencontre cette «bonne épouse » qui s'efforce de rester une bonne personne : son éthique et sa morale deviennent de plus en plus anachroniques, sans qu'elle consente véritablement à s'en détacher, créant là de classiques dissonances cognitives. Pour solution, l'hérö̈ne se radicalise peu à peu car elle prend conscience qu'il ne suffit pas d'investir la sphère publique (même si la confiance en elle qu'elle tire de ses accomplissements professionnels agit bien sûr au-delà du bureau et jusque dans ses relations amoureuses), mais qu'il lui faut encore se positionner comme sujet actif et compétent dans la sphère privée, et dans les échanges entre public et privé. Ainsi, de façon intéressante, le féminisme libéral se révèle rapidement obsolète et est vite rattrapé par son adversaire historique, le féminisme radical, qui propose des outils plus efficaces pour penser la détraditionnalisation : critique systémique du couple et refonte de l'organisation privé/public.

\section{Victoires et échecs d'une relation pure}

En parallèle de la détraditionnalisation qui se produit avec son mari, l'héroïne s'engage dans une relation à visée égalitaire, et dont la sexualité est une clé de voûte, avec le personnage de Will Gardner. Il s'agit là d'un territoire évidemment déjà bien investi depuis Sex and the City, mais dont il faut souligner que la liberté de ton était encouragée par sa diffusion sur $\mathrm{HBO}$, c'est-à-dire en dehors de la législation de la FCC (équivalent du CSA français). Les règles de bienséance imposées aux networks sont beaucoup plus strictes que sur le câble, faisant de l'autosuffisance du plaisir charnel et de la positivité de la sexualité une démocratisation relativement inédite. Cet ars erotica est développé par l'héroïne dans sa relation avec Will mais elle n'est pas l'exclusivité de ce partenaire (elle performera le même type d'aisance sexuelle avec son mari dans les épisodes ultérieurs). 
Alicia Florrick, qui était durant les premières saisons un personnage peu sexué, construit à cette occasion une sexualité plastique, orientée vers le plaisir, ayant lieu hors de la chambre à coucher et ne se limitant plus à des positions classiques. La sexualité devient un territoire réapproprié, sur lequel exprimer son indépendance mais surtout d'où l'on

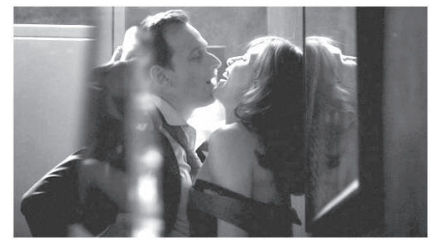
peut tirer une assurance renouvelée qui servira d'autres sphères d'activité de l'individu. Là encore, la porosité entre sphère publique et sphère privée est instrumentalisée au profit des identités. Faisant l'amour, Alicia et Will s'amusent ainsi de jeux de mots, chargeant d'une signification sexuelle un vocabulaire qui est traditionnellement celui du travail :

Alicia : - On en fait trop ?

Will : - Diane (son associée) pense que je suis trop dur avec toi. Je le suis ? Trop dur ? (Elle rit.) Toutes ces nuits...

Alicia : - ... sans aucune pause...

WILL : - ... croulant sous le travail...

Alicia : - ... à genoux!

$(\text { Ils rient. })^{3}$

Ce faisant, The Good Wife a cette particularité de ne pas réduire l'amour ou la sexualité à des moments affectifs ou érotiques, mais d'en explorer les conséquences positives sur la confiance en soi d'une héroïne qui tourne la porosité public/privé en excitation sexuelle, elle-même expression d'une énergie de vie redécouverte. In fine, la relation pure telle que représentée dans cette série est une bulle intime dans laquelle les amoureux se réinventent ensemble. Leur relation est donc, à l'instar du modèle décrit par Anthony Giddens, profondément contingente : elle ne perdure que parce que les partenaires sont visiblement heureux, respectant chacun le territoire tant professionnel que personnel de l'autre, acceptant qu'il ne soit pas toujours prêt à partager certains pans de sa vie. Lorsque Will se voit essuyer un refus après avoir proposé à Alicia de rencontrer ses enfants, il est visiblement blessé mais n'insiste pas plus avant. Ainsi, alors que l'amour entre Alicia Florrick et son mari soutient en réalité une structure traditionnelle qui voit en l'émancipation féminine une menace, il est entre Alicia et Will un encouragement à l'indépendance personnelle, condition 
sine qua non au bonheur interpersonnel. Ce sera pourtant précisément cette contingence qui mènera la relation à sa perte. Alors qu' il ne fait aucun doute que l'héroïne est amoureuse de Will, elle rompt pour préserver ses enfants : incapables de formaliser (à défaut d'officialiser) le couple dans la sphère publique, celui-ci ne résiste pas aux impératifs familiaux que s'impose Alicia. L'éthique bourgeoise est résiduelle, elle est constamment interrogée et critiquée, mais ses effets restent puissants.

Toutefois, l'innovation de ces représentations est que la relation pure a ici la particularité de ne pas dépendre d'un seul partenaire, mais d'être le résultat des nouvelles identités, ce qui amène l'hérö̈ne à conditionner sa réconciliation avec son mari à ces nouvelles valeurs. Durant la quatrième saison, Alicia Florrick apparaît particulièrement sûre d'elle, performant une sexualité épisodique avec son époux et ne se laissant plus prendre au piège d'un lyrisme romantique dont elle sait désormais qu'il cache un patriarcat sévère. Qu'elle soit donc en couple avec Will Gardner ou qu'elle retourne avec son mari, l'héroïne apparaît peu à peu comme une redoutable partenaire amoureuse, capable de mobiliser des compétences diverses. De son mariage bourgeois, l'ancienne femme au foyer a conservé une intelligence émotionnelle très développée, à laquelle elle adjoint, grâce à sa reconquête du métier d'avocate, un agir stratégique, un fort savoir-faire rhétorique et une assurance nouvelle. Ce cocktail permet le déploiement d'un comportement jusqu' ici réservé au masculin : la sexualité est un plaisir personnel et le langage amoureux n'est plus adossé à l'oblativité. Cela est représenté sans que la hantise des mouvements anti-égalitaires ne se produise : Alicia Florrick ne retourne pas la domination, comme pouvaient le faire Roseanne et Maude durant les années 1980 ou, plus récemment, l'héroïne de Nurse Jackie, mais redistribue le pouvoir grâce à des micropolitiques savamment dosées.

Si The Good Wife marque un tournant si majeur, c'est donc parce qu'elle représente un enchevêtrement complexe des politiques amoureuses et des politiques de la deuxième modernité : point d'émancipation totale, point d'identités euphoriques car joyeusement libérées, point d'égalité parfaite, mais une réflexion élaborée de la part d'une héroïne sur les tromperies et les abus précédemment subis. L’objectif très particulier est celui du refus de la victimisation, en vue d'un empowerment stable. Cette vision d'un pouvoir diffus et non résultant d'une causalité simple explique que l'émancipation 
représentée soit plus lente qu'à l'accoutumée. De la conquête de la sphère professionnelle à l'élaboration de relations pures, de la pudeur bourgeoise à l'identité sexuelle, de l'intelligence émotionnelle à l'intelligence émotionnelle et stratégique, les métamorphoses d'Alicia Florrick incarnent finalement un demi-siècle d'émancipation féminine. Celui-ci ne se fait ni dans une allégresse naïve ni dans une ascèse amère mais dans des mouvements de progression et de reculade dus aux manifestations plurielles du pouvoir. Qu'une héroïne à priori conservatrice soit un tel avatar de l'émancipation féminine est donc tout sauf un paradoxe : le monde post-romantique ne peut advenir qu'après avoir formulé une critique minutieuse du monde romantique.

1 «The Good Wife: Non-Lawyers Behind That Lawyer Show », Bitter Lawyer, 4 janvier 2010. Disponible en ligne : http://www.bitterlawyer. $\mathrm{com} /$ the-good-wife-non-lawyers-behind-that-lawyer-show/.

2 The Good Wife, saison 2, épisode 23.

3 The Good Wife, saison 3, épisode 1. 



\section{Conclusion}

Cinquante ans se sont écoulés entre les fugues répétées de Lucy Ricardo et le désespoir enfin exprimé de Lynette Scavo. Quarante ans sont passés depuis les pouvoirs d'une sorcière bien-aimée, métaphore de capacités d'agir bridées, jusqu'à ceux d'une Tueuse de vampires qui la condamnent à la solitude. Dix ans seulement séparent l'avocate névrosée Ally McBeal, qui rêve d'un couple absolument fusionnel, de la « bonne épouse » Alicia Florrick, femme bourgeoise qu'une tromperie rendue publique a reconvertie en brillante avocate et en amoureuse stoïque. De 1950 à 2010, soixante ans de séries télévisées donnent à voir des amours romantiques, des familles nucléaires et des sexualités libérées qui sèment ou contrarient des émancipations féminines, rendues contradictoires par le syncrétisme des mythologies médiatiques.

Quatre axes interdépendants structurent les récits sur les amours des héroïnes : les résistances patriarcales y passent de l'arbitraire contesté à la négociation ; les manières féminines d'aimer s'affranchissent du sacrifice ; la communication amoureuse se charge d'une visée démocratique; enfin, les sphères publique et privée opèrent des réarticulations. Cet ensemble témoigne de querelles de significations autour des modes d'émancipation féminine, des modèles amoureux et des modalités communicationnelles, tant en ce qui concerne les sentiments ressentis par les personnages que les formes démocratiques élaborées par les couples fictionnels. Ces grands pôles se cristallisent, ces dernières années, dans une androgynisation des manières d'aimer, dans la réapparition de problèmes autrefois soulevés par le féminisme radical, dans la réconciliation du féminisme et de l'individualisme, et enfin dans l'ambivalence de la démocratie relationnelle, tiraillée entre l'idéal d'une intercompréhension et l'expression de désaccords amoureux. 
Le modèle romantique perd du terrain face à la relation pure, en ce que les héroïnes trouvent dans la sexualité plastique et dans la contingence qui la caractérisent la possibilité d'exprimer leur individualité tout en la dépassant pour se lier affectivement à autrui.

Au final, le recul dont ont fait preuve les récits fictionnels dès leurs débuts (ainsi, les évasions de Lucy Ricardo ou la conversion discutée de Samantha Stevens à l'idéologie du rêve américain) incite à infléchir l'hypothèse de séries qui n’auraient gagné en complexité et en critique sociale que depuis deux décennies. On peut, en revanche, voir dans la recrudescence, depuis 2004, des femmes parmi les créateurs de séries télévisées et plus encore dans la structuration d'une stratégie de programmation autour d'elles, une stabilisation des contre-publics subalternes féminins, dont il faut toujours rappeler la diversité. Ces mutations industrielles ne sont pas étrangères à la radicalisation des revendications féministes des héroïnes. Cela interroge l'épuisement provoqué par une gestion capitaliste de la sphère professionnelle (Nurse Jackie), la sous-responsabilisation des hommes (The Good Wife, The Big C), l'hypocrisie de la représentation du rêve américain (Desperate Housewives, Weeds) ou encore l'obsolescence programmée des valeurs néoféministes (Cougar Town, The New Adventures of Old Christine).

En premier lieu, la binarité du système de genre et l'androgynisation de la communication des sentiments se font jour. L'oblativité féminine était omniprésente dans les premières représentations médiatiques, qu'elle fut exigée d'un féminin réticent (I Love Lucy) ou actée de bon cœur (Ma Sorcière bien-aimée, The Donna Reed Show), mais elle sort très bouleversée de ce demi-siècle. Caractérisée d'injuste par des avatars du féminisme libéral (The Mary Tyler Moore Show, Murphy Brown) comme du féminisme radical (Maude, Roseanne), cette façon spécifiquement féminine d'aimer est invisibilisée au cours des années 1990 avant d'être reconvoquée dans les années 2000 par des héroïnes qui, l'ayant pratiquée pendant quinze ans au sein d'un mariage bourgeois, en dressent une critique acerbe. Au cours de cette évacuation progressive, se posent successivement la question de ce qui construit l'injonction au sacrifice féminin, puis le problème des nouvelles formes communicationnelles qui le remplacent.

Le problème des répercussions de l'émancipation sur les manières d'aimer est le privilège d'héroïnes blanches et issues de classes supérieures, mais l'une des surprises de cette recherche est que, alors que les 
classes populaires restent sous-représentées, les séries télévisées ont depuis soixante ans spécifiquement abordé la variabilité des comportements en fonction de l'origine sociale des personnages féminins. D'un côté, dans Roseanne en 1998 comme dans Nurse Jackie en 2009, le dialogue conjugal est représenté comme un travail relationnel que l'ouvrière ou l'infirmière, épuisée par sa journée, n’a plus le courage de mener. Toutefois, en l'espace de dix ans, un changement majeur s'est produit : tandis que Roseanne Connor, explicitement féministe, requérait sans succès de son époux une plus grande participation aux tâches domestiques, Jackie Peyton a pour mari un homme doux et attentionné, qui s'occupe très largement de l'éducation des enfants. Ce passage d'une critique de l'organisation patriarcale inspirée du féminisme radical (Roseanne) à une critique du partage dichotomique entre public et privé (Nurse Jackie) délie les difficultés amoureuses de la seule idéologie patriarcale pour les envisager comme des conséquences socioéconomiques de l'organisation sociale industrielle.

D'un autre côté, dans Dharma \& Greg (1997) ou dans Gilmore Girls (2000), les ethos bourgeois sont critiqués avec force : ils sont des dispositions comportementales inauthentiques qui entravent le bon déroulement de la démocratie relationnelle. Les inflexions que fait subir le personnage hippie de Dharma au surmoi bourgeois de son partenaire sont la première occurrence d'une relation pure tournée vers l'intercompréhension, rendue possible par l'exotisme de l'idéologie new age. De façon plus implicite, le déclassement que subissent des héroïnes quadragénaires à partir de 2004 (Desperate Housewives, Weeds, The Good Wife, The New Adventures of Old Christine) est une conséquence de l'effondrement de leur mariage. Là encore, le capitalisme qui sous-tend le rêve américain s'écroule en même temps que le romantisme qui sous-tend le mariage bourgeois : le grand partage industriel public-masculin/privé-féminin qui a historiquement succédé aux sociétés traditionnelles produit des dysfonctionnements trop lourds pour être solubles dans de simples réformes qui préserveraient ces dichotomies. L'obsolescence du romantisme est aussi celle de l'amour oblatif caractéristique d'un partage supposément fonctionnel du public et du privé mais aveugle aux aspirations individuelles.

Venant à la rescousse d'héroïnes qui se demandent comment prouver leur amour sans se sacrifier, la sexualité devient une particule élémentaire de l'univers amoureux. Toutefois, comme l'a bien montré Gayle Rubin, le 
renouvellement de son expression n'implique pas nécessairement celui du genre : tout en se réappropriant des comportements masculins, les hérö̈nes restent indubitablement féminines. Dans les séries, les corps féminins sont soumis au système binaire de genre : les codes sociaux (vêtements, coiffures, artifices) sont traditionnellement répartis tandis que les normes corporelles s'expriment dans la beauté et la minceur. Quelques exceptions parsèment un trouble dans le genre ou une subversion des canons mais qui ne laissent pour autant planer aucun doute sur l'appartenance à la catégorie féminine : les cheveux courts restent blonds (Nurse Jackie, The Big C), les rides des quadragénaires sont entourées d'un brushing impeccable (The Good Wife) et la laideur supposée de la récente mère de famille relève autant d'une mascarade que sa précédente beauté sophistiquée (Gabrielle Solis, Desperate Housewives). La déconstruction du genre est pour l'heure contenue à son exagération : le néoféminisme des héroïnes, qui revalorise le genre féminin, produit des retournements de stigmate sous forme là aussi de mascarades de genre. L'espionne Sydney Bristow fait croire à une naïveté pour mieux désarmer ses ennemis tandis que les balades citadines de Sex and the City se transforment en défilés de mode. Une nouvelle objectivation, volontaire et stratégique, est instrumentalisée par les femmes pour paradoxalement dépasser la stigmatisation. Elles n'échappent évidemment pas à certains pièges, au premier rang desquels le phénomène de «double standard » : leur obstination à performer un genre féminin crée en retour des attentes affectives classiques. Des propositions médiaculturelles évoquent depuis peu, en concomitance avec l'apparition de femmes divorcées, l'obsolescence de cette stratégie : des séries comme The New Adventures of Old Christine ou Cougar Town évoquent les pertes de repère de ces femmes qui voient avec effroi leur jeunesse s'évaporer. La dénaturalisation des genres, à défaut de leur déconstruction encore très lointaine, affleure plutôt dans les comportements. Depuis la rupture effectuée par Sex and the City à la fin des années 1990, un travail de resignification opère autour de la sexualité, entendue comme un ars erotica propice à l'épanouissement des femmes. L'amour devient indissociable de la sexualité mais, en retour, cette dernière s'autonomise car les hérö̈nes sont affranchies des craintes de la reproduction, cherchent le plaisir physique et objectivent les corps de leurs partenaires. Cette démocratisation des comportements jusqu'ici réservés au masculin androgynise les manières d'aimer en même temps qu'elle 
redonne aux corps un potentiel expressiviste. L'amour ne se contente plus de la rencontre des esprits, il faut encore qu'elle soit celle des chairs.

Une deuxième question que soulève ce retour sur soixante ans de séries télévisées étasuniennes est l'étonnante revanche que prend le féminisme radical. L'optimisme de l'émancipation individualiste, représentée de Murphy Brown jusqu'à Sex and the City, empêchait, non pas forcément de penser les effets de structure, mais de les représenter en action. Cette vague atteint son plus haut point dans les années 1990 avec des personnages trentenaires qui profitent de l'anonymat de la grande ville (symbole de la conquête de la sphère publique permise par le féminisme libéral) pour expérimenter des relations amoureuses et sexuelles euphoriques, mais elle est suivie d'héroïnes quadragénaires, épouses bourgeoises et mères au foyer, qui se retrouvent propulsées malgré elles hors du mariage et dans l'émancipation féminine. Depuis 2004, les séries réintègrent la dimension structurelle de l'oppression patriarcale qui faisait défaut à bien des fictions dans les années 1980 et 1990. Lors de l'effondrement de leur mariage, ces héroïnes sont dépossédées de capitaux sociaux et de capitaux financiers, mais pas de capacités d'agir. Fortes d'une émancipation qu'elles n'ont pas désirée, ces femmes réinvestissent la sphère professionnelle en même temps qu'elles redéfinissent la sphère amoureuse, portées par la découverte de valeurs féministes. Le dialogue entre public et privé se fait alors de façon consubstantielle, et non plus successive ou concurrentielle, allant à certains endroits jusqu'à remettre en cause cette distinction même. Ce faisant, ces héroïnes permettent de replacer au cœur des récits sur l'amour une critique de l'hégémonie patriarcale de la première modernité. Curieusement, le féminisme radical que l'on donnait définitivement battu au sortir des années 1970 par le féminisme libéral, s'engouffre dans la porte qu'ouvre ce réagencement de la sphère publique et de la sphère privée : une critique systémique de l'ère patriarcale qu'ont vécue les héroïnes quadragénaires conduit à un processus minutieux de détraditionnalisation.

Les protagonistes de Desperate Housewives, Weeds, The Good Wife, The New Adventures of Old Christine, Cougar Town ou encore The Big C prennent en effet conscience - thématique classique du féminisme radical des inégalités structurelles qui pesaient et continuent de peser sur elles : les disparités économiques du mariage de la première modernité restreignent leurs capacités d'agir (Desperate Housewives, Weeds, The Good Wife) tandis 
que les inégalités communicationnelles de l'amour deviennent de moins en moins tolérables. Le néoféminisme, si influent auprès des hérö̈nes trentenaires, devient également un ressort comique produit aux dépens des héroïnes qui le vivent : jusque dans les titres, la « cougar » Jules Cobb (Cougar Town) ou la «vieille » Christine (The New Adventures of Old Christine) incarnent le problème de ce mouvement féministe passé quarante ans. Le retour à la sphère privée, qui peut sembler être un backlash, est en fait un réinvestissement par les héroïnes d'un territoire auquel elles ont été confinées mais qu'elles n'ont que peu souvent défini.

La rupture qu'incarne à cet égard Alicia Florrick, l'héroïne de The Good Wife, tient à ce qu'elle produit de nombreuses redéfinitions de son mariage et que celui-ci ne survit à aucune de ces redistributions du pouvoir (affectif comme sexuel ou professionnel). Première vraie rupture au féminin de la télévision étasunienne, The Good Wife est l'indice d'une percée dans la sphère publique d'héroïnes qui, enfin, exercent du pouvoir pour définir l'amour, et non plus contestent, négocient ou acceptent une hégémonie patriarcale. Condition de ce nouvel ordre amoureux, les séries télévisées représentent une recapitalisation des compétences : les femmes tirent à la fois de leur assignation passée à la sphère privée de fortes aptitudes émotionnelles tandis que leur choc émancipateur leur offre une confiance en elles que les héroïnes des décennies précédentes avaient peu (ou payaient d'un désert affectif). Cette insistance des imaginaires médiatiques pour les phénomènes de recapitalisations provient évidemment d'une influence capitaliste mais est aussi le signe d'une déliquescence des frontières entre les sphères d'activité. À l'optimisme de l'individualisme expressiviste des années 1990, succède l'optimisme d'échanges semble-t-il infinis entre le public et le privé. Les réarticulations entre la sphère publique et la sphère privée rythment donc l'histoire des représentations des amours à tel point que, dans The Good Wife, la dichotomie public/privé ne fait plus sens que stratégiquement. In fine, ces différentes articulations ne sont plus légitimes en elles-mêmes mais agissent comme la possibilité d'explorer les différentes façons d'être à la fois un individu émancipé, un sujet féministe et une femme amoureuse.

Les promesses de la relation pure sont finalement celles d'une égalité de genre atteinte par des valeurs et des techniques communicationnelles démocratiques. Au fil des représentations, la communication conjugale se heurte à la divergence d'opinions des partenaires. Signe d'un affaiblissement de 
l'autorité masculine, dans certaines séries, les tours de passe-passe ne sont plus seulement ceux des femmes opprimées qui fuguent ou complotent pour échapper au joug de leur époux, mais proviennent des personnages masculins qui manigancent pour obtenir ce qu' ils veulent (Desperate Housewives). Leur pouvoir ayant perdu le privilège de l'arbitraire, ils se réfugient dans des formes de manipulations stratégiques ou émotionnelles - signe également de la prérogative que prennent ces communications dans la modernité avancée. Autre indice du retour d'arguments féministes radicaux, la critique systémique portée par les héroïnes enjoint aux personnages masculins de repenser leur propre position. Ceux-ci ne passent plus à travers les filets du féminisme comme c'était le cas des partenaires des héroïnes trentenaires mais font désormais intégralement partie du problème féministe. Les réconciliations des partenaires ne peuvent se faire qu'au prix d'une refonte en profondeur de leur couple : tournant vers l'agapè dans The Big C, redéfinitions contingentes dans The Good Wife, prévalence de l'honnêteté dans Desperate Housewives. Des régimes discursifs démocratiques étayent ces valeurs : négociations, compromis, concessions ou renoncements volontaires affleurent, construisant dans les médiacultures des « sphères publiques intimes » qui prennent pour défi de mettre en lien amoureux sans nier les individualités si laborieusement construites. Ces nouveaux imaginaires médiaculturels s'inscrivent dans une modernité avancée, attentive aux risques amoureux : les hérö̈nes rationalisent l'amour, dont elles embrassent les politiques, tout en craignant les répercussions de leurs choix individuels et affectifs. Si sentiments et sexualités ont trouvé des réarticulations égalitaires depuis les années 1990, il semble que les héroïnes quadragénaires marquent une nouvelle étape en ambitionnant d'en faire de même entre sentiments individuels et structures conjugales.

Que devient finalement le modèle romantique, pris entre les deux feux de l'émancipation féminine et de la reformulation du public et du privé ? Au cours des années 1990, les héroïnes trentenaires étaient prises au piège de la double injonction de la réalisation de soi et de l'accomplissement conjugal, et confrontées de surcroît aux difficultés d'ajuster les prérequis de ce modèle à la réalité des pratiques.

Le romantisme s'était relativement bien adapté à l'apparition d'héroïnes indépendantes (The Mary Tyler Moore Show, Murphy Brown, Sex and the City, Ally McBeal, Gilmore Girls, Alias...) : les élans intuitifs, la croyance en 
une prédestination amoureuse, la volonté de se fondre en l'autre ont fortement résisté jusqu'à l'orée des années 2000. Ces modalités communicationnelles romantiques restaient probablement d'autant plus prégnantes que ces personnages de femmes actives cherchaient, à la manière des hommes qui les ont précédées, à faire de la sphère amoureuse un refuge. Toutefois, dans le même temps, leur conquête de la sphère publique permettait le développement d'un individualisme (confiance en elle, affirmation de soi, impératif égalitaire) qui portait un coup sérieux aux prérogatives sacrificielles du romantisme. Pour concilier ces contraires, les séries télévisées ont trouvé une solution dans la représentation d'une grande fracture entre imaginaires romantiques et pratiques de la relation pure. Il est intéressant que les fictions utilisent cette contradiction pour évoquer la complexification des relations amoureuses engendrée par leur relatif décollement des structures maritales. Avec la fin des modèles holistes, s'écroule également le mythe d'une distinction ferme entre ce qui est rêvé et ce qui est pratiqué : en représentant de la sorte les héroïnes de l'après-féminisme, les séries réhabilitent la portée « réelle » des imaginaires amoureux, entendus au sens large des rêves, des utopies, des fictions ou des mythes dont on interroge désormais l'influence sur les pratiques. Cette préséance que gagnent les imaginaires auprès des héroïnes trentenaires a certainement participé de la dépréciation de ces séries par des auteur.e.s qui appellent de leurs vœux une représentation plus matérialiste des inégalités de genre. Il faut attendre les héroïnes quadragénaires des années 2000 pour que la sphère privée ne soit plus définie par la mythologie, historiquement masculine, du cocon. La trahison du romantisme que vivent ces personnages déconstruit l'idéologie unificatrice et apaisante de ce modèle : les politiques genrées qui y sont à l'œuvre se révèlent de la façon la plus violente qui soit puisque ces héroïnes sont soudainement privées d'ami.e.s (qui étaient du cercle de leur époux) et de revenus. Le procédé narratif d'un événement « choc » est l'occasion d'une redécouverte des courants féministes des années 1960 et 1970 qui doivent composer avec l'individualisme réflexif.

Au bonheur des âmes sœurs, heureuses car mêlées l'une à l'autre, succède le bonheur des individus, heureux en couple car encouragés et reconnus par lui. La conséquence de ces renversements est que les héroïnes ne rêvent plus de balayer les rapports de pouvoir : contrairement aux personnages de la période 1997-2004 (Sex and the City et Ally McBeal en tête) qui espèrent que 
les formes embryonnaires de relation pure deviennent des amours romantiques, les femmes quadragénaires qui peuplent la fiction télévisée depuis 2004, parce qu'elles ont été déçues par le modèle romantique, ne sont plus dupes du fait que le pouvoir est intrinsèque aux formes amoureuses. Peu à peu, les imaginaires médiatiques placent au cœur du couple le travail communicationnel qui le forge. L'utopie de la relation romantique, qui se basait sur des communications que la prédestination rendait intuitives, s'affaiblit. Elle est remplacée par une autre utopie, celle d'une relation égalitaire dont l'intercompréhension est nourrie par l'épanouissement personnel. Dans l'apparition de ces « sphères publiques intimes », les femmes se positionnent comme sujets longtemps opprimés et comme individus sur la voie d'une émancipation qui ne pourra se faire qu'à condition de repenser de façon systémique l'organisation de la sphère privée. En ce sens, la réconciliation du féminisme et de l'individualisme, longtemps pensés contradictoires, se fait sur les cendres par endroits encore chaudes du romantisme. 



\section{Bibliographie}

\section{Ouvrages et chapitres d'ouvrages}

Akass, Kim, McCabe, Janet, Reading Sex And The City, Londres, I.B. Tauris, 2003.

Allen, Robert C., Speaking of Soap Opera, Chapel Hill, University of North Carolina Press, 1985.

Badiou, Alain, Éloge de l'amour, Paris, Flammarion, 2011.

Bailey, Beth, From Front Porch to Back Seat. Courtship in TwentiethCentury America, Baltimore, Johns Hopkins University Press, 1989.

Bakhtine, Mikhail, L'euvre de François Rabelais et la culture populaire au Moyen-Age et sous la Renaissance, Paris, Gallimard, 1970.

Ball, Lucille, Love, Lucy, New York, Putnam, 1996.

Bauman, Zygmunt, L'Amour liquide. De la fragilité des liens entre les hommes, Paris, Fayard, 2010.

Bathrick, Serafina, « The Mary Tyler Moore Show. Women at Home and at Work », in Morreale, Joanne, Critiquing the Sitcom. A Reader, Syracuse, Syracuse University Press, 2002.

Beauvoir (de), Simone, Le Deuxième sexe. Tome 1 : Les faits et les mythes, Paris, Gallimard, 1986.

Beauvoir (de), Simone, Le Deuxième sexe. Tome 2, Paris, Gallimard, 1986. Beck, Ulrich, Reflexive Modernization: Politics, Tradition and Aesthetics in the Modern Social Order, Palo Alto, Stanford University Press, 1994.

Beck, Ulrich, Beck-Gernsheim, Elizabeth, The Normal Chaos of Love, Cambridge, Polity Press, 1995. 
Beck, Ulrich, La Société du risque. Sur la voie d'une autre modernité, Paris, Flammarion, 2001.

Bilat, Loïse, Haver, Gianni, Le héros était une femme..., Paris, Antipodes, 2011.

Boltanski, Luc, L'Amour et la Justice comme compétences, Paris, Gallimard, 1990.

Bourcier, Marie-Hélène, Comprendre le féminisme, Paris, Max Milo, 2012.

Bourdieu, Pierre, La Domination masculine, Paris, Seuil, 2002.

Braham, Peter, Allen, John, Lewis, Paul, Hall, Stuart, Political and Economic Forms of Modernity, Cambridge, Polity Press, 1992.

Brooks, Ann, Postfeminisms. Feminism, Cultural Theory and Cultural Forms, Londres, Routledge, 1997.

Brunsdon, Charlotte, D’Acci, Julie, Spigel, Lynn, Feminist Television Criticism. A Reader, Oxford, Oxford University Press, 1997.

Burch, Noël, De la beauté des latrines. Pour réhabiliter le sens au cinéma et ailleurs, Paris, L'Harmattan, 2012.

Butler, Judith, Défaire le genre, Paris, Amsterdam, 2006.

Butler, Judith, Trouble dans le genre. Pour un féminisme de la subversion, Paris, La Découverte, 2005.

Cancian, Francesca, Love In America. Gender and Self-Development, Cambridge, Cambridge University Press, 1991.

Carson, Diane, Dittmar, Linda, Welsch, Janice R., Multiple Voices in Feminist Film Criticism, Minneapolis, University of Minnesota Press, 1993.

Cavell, Stanley, Pursuits of Happiness. The Hollywood Comedy of Remarriage, Cambridge, Harvard University Press, 1984.

Chaumier, Serge, L'Amour fissionnel. Le nouvel art d'aimer, Paris, Fayard, 2004.

Chaumier, Serge, La Déliaison amoureuse. De la fusion romantique au désir d'indépendance, Lausanne, Payot, 2004.

Clark, Danae, « Cagney \& Lacey: Feminist Strategies of Detection », in Brown, Mary Ellen, Television and Women's Culture: The Politics of the Popular, Londres, Sage Publications, 1990.

D'Acci, Julie, Defining Women: Television and the Case of Cagney \& Lacey, Chapel Hill, The University of North Carolina Press, 1995.

Davis, Flora, Moving the Mountain. Women's Movement in America since 1960, Upper Saddle River, Prentice Hall, 1991. 
Delphy, Christine, L'Ennemi principal. Tome 2 : Penser le genre, Paris, Syllepse, 2001.

Delphy, Christine, L'Ennemi principal. Tome 1 : Économie politique du patriarcat, Paris, Syllepse, 2002.

Digby, Tom, Bartky, Sandra, Men Doing Feminism, Londres, Routledge, 2008.

Doty, Alexander, « I Love Laverne and Shirley. Lesbian Narratives, Queer Pleasures, and Television Sitcoms », in Morreale, Joanne, Critiquing the Sitcom. A Reader, Syracuse, Syracuse University Press, 2002.

Douglas, Susan J., Where the Girls Are: Growing Up Female with the Mass Media, New York, Three Rivers Press, 1995.

Dow, Bonnie J., Prime-Time Feminism. Television, Media Culture, and the Women's Movement Since 1970, Philadelphia, University of Pennsylvania Press, 1996.

Dow, Bonnie, « "How Will You Make It On Your Own?” Television and Feminism since 1970 », in Wasko, Janet, A Companion to Television, Hoboken, Wiley-Blackwell, 2009.

Echols, Alice, Daring to be Bad. Radical Feminism in America. 1967-1975, Minneapolis, University of Minnesota Press, 1989.

Eco, Umberto, «The Frames of Comic "Freedom" », in Eco, Umberto, Ivanov, V.V., Recto, Monica, Carnival!, Berlin, De Gruyter, 1984.

Ehrenberg, Alain, La Fatigue d'être soi. Dépression et société, Paris, Odile Jacob, 2000.

Esquenazi, Jean-Pierre, Les Séries télévisées : l'avenir du cinéma?, Paris, Armand Colin, 2010.

Faludi, Susan, Backlash. La guerre froide contre les fermes, Paris, Des Femmes, 1993.

Farber, David, Bailey, Beth, The Columbia Guide to America in the 1960s, New York, Columbia University Press, 2001.

Fiske, John, Television Culture, Londres, Routledge, 1994.

Fiske, John, « Murphy Brown, Dan Quayle, and the Family Row of the Year », in Media Matters: Everyday Culture and Political Change. Race and Gender in U.S. Politics, Minneapolis, University of Minnesota Press, 1996.

Foucault, Michel, L'Ordre du discours, Paris, Gallimard, 1971.

Foucault, Michel, Dits et écrits. Tome 1 : 1954-1975, Paris, Gallimard, 2001. 
Foucault, Michel, Dits et écrits. Tome 2 : 1976-1988, Paris, Gallimard, 2001.

Fraser, Nancy, Qu'est-ce que la justice sociale?, Paris, La Découverte, 2005. Freeland, Cynthia, «Feminist Film Theory », in Kelly, Michael, Encyclopedia of Aesthetics, Oxford, Oxford University Press, 1998.

Freud, Sigmund, Essais de psychanalyse, Lausanne, Payot, 2004.

Friedan, Betty, La Femme mystifiée, Paris, Gonthier, 1963.

Fultz, Jay, In Search of Donna Reed, Chicago, University of Iowa Press, 2001. Gallagher, Margaret, «Feminist Media Perspectives », in Valvidia, Angharad, A Companion to Media Studies, Hoboken, Wiley-Blackwell, 2005.

Gamble, Sarah, The Routledge Companion to Feminism and Postfeminism, Londres, Routledge, 2002.

Gauntlett, David, Media, Gender, and Identity: An Introduction, Londres, Routledge, 2008.

Genz, Stephanie, Brabon, Benjamin, Postfeminism. Cultural Texts and Theories, Edinburgh, Edinburgh University Press, 2009.

Geraghty, Christine, Women and the Soap Opera. A Study of Prime Time Soaps, Cambridge, Polity Press, 1990.

Geraghty, Christine, « The Study of the Soap Opera », in Wasko, Janet, A Companion to Television, Hoboken, Wiley-Blackwell, 2005.

Gerbner, George, Signorielli, Nancy, Women and Minorities in Television Drama. 1969-1978, Philadelphie, University of Philadelphia, Annenberg School of Communications, 1979.

Giddens, Anthony, Modernity and Self-Identity: Self and Society in the Late Modern Age, Palo Alto, Stanford University Press, 1991.

Giddens, Anthony, « Living in a post-traditional society », in Beck, Ulrich, Reflexive Modernization: Politics, Tradition and Aesthetics in the Modern Social Order, Palo Alto, Stanford University Press, 1994.

Giddens, Anthony, Pierson, Christopher, Conversations with Anthony Giddens. Making Sense of Modernity, Palo Alto, Stanford University Press, 1998.

Giddens, Anthony, La Transformation de l'intimité. Sexualité, amour, et érotisme dans les sociétés modernes, Rodez, Éditions du Rouergue, 2004. Gilroy, Paul, The Black Atlantic: Modernity and Double Consciousness, Brooklyn, Verso, 1993. 
Gilroy, Paul, Against Race: Imagining Political Culture Beyond the Color Line, Cambridge, Harvard University Press, 2000.

Glevarec, Hervé, Macé, Éric, Maigret, Éric, Cultural Studies. Anthologie, Paris, Armand Colin, 2008.

Goffman, Erving, La mise en scène de la vie quotidienne. Tome 1. La présentation de soi, Paris, Les Éditions de Minuit, 1973.

Goffman, Erving, La mise en scène de la vie quotidienne. Tome 2. Les relations en public, Paris, Les Éditions de Minuit, 1973.

Greer, Germaine, La Femme eunuque, Paris, Robert Laffont, 1971.

Grossberg, Larry, We Gotta Get Out Of This Place. Popular Conservatism And Postmodern Culture, Londres, Routledge, 1992.

Grossberg, Larry, Cultural Studies and the Future Tense, Durham, Duke University Press Books, 2010.

Guénif-Souilamas, Nacira, Macé, Éric, Les Féministes et le garçon arabe, La Tour d'Aigues, L'Aube, 2006.

Gurley Brown, Helen, Sex and the Single Girl, New York, Bernard Geis Associates, 1962.

Habermas, Jürgen, Théorie de l'agir communicationnel, Paris, Fayard, 1987.

Hall, Stuart, «Culture, The Media and the "Ideological Effect" », in Curran, James, Gurevitch, Michael, Woollacott, Janet, Mass Communication and Society, Londres, Sage Publications, 1979.

Hall, Stuart, « Who Needs Identity? », in Hall, Stuart, Du Gay, Paul, Questions of Cultural Identity, Londres, Sage Publications, 1996.

Hall, Stuart, Representation. Cultural Representations And Signifying Practices, Londres, Sage Publications, 1997.

Hall, Stuart, Identités et cultures. Politiques des cultural studies, Paris, Amsterdam, 2008.

Haralovich, Mary Beth, « Sitcoms and Suburbs: Positioning the 1950s Homemaker », in Spigel, Lynn, Mann, Denise, Private Screenings. Television and the Female Consumer, Minneapolis, University of Minnesota Press, 1992.

Haralovich, Mary Beth, Rabinovitz, Lauren, Television, History, And American Culture. Feminist Critical Essays, Durham, Duke University Press, 1999.

Hardt, Michael, Negri, Antonio, Multitude. Guerre et démocratie à l'âge de l'Empire, Paris, 10/18, 2006. 
Hayden, Dolores, Redesigning the American Dream. The Future of Housing, Work, and Family Life, New York, W.W. Norton \& Company, 2002. Hoggart, Richard, La Culture du pauvre. Étude sur le style de vie des classes populaires en Angleterre, Paris, Les Éditions de Minuit, 1970.

hooks, bell, Feminist Theory From Margin To Center, New York, South End Press, 1984.

hooks, bell, « The Oppositional Gaze: Black Female Spectators », in Thornham, Sue, Feminist Film Theory: A Reader, New York, New York University Press, 1999.

Huyssen, Andreas, After the Great Divide: Modernism, Mass Culture, Postmodernism, Bloomington, Indiana University Press, 1987.

Huyssen, Andreas, « Féminité de la culture de masse, l'autre de la modernité », in Sellier, Viennot, Culture d'élite, culture de masse et différence des sexes, Paris, L'Harmattan, 2004.

Illouz, Eva, Les Sentiments du capitalisme, Paris, Seuil, 2006.

Illouz, Eva, Why Love Hurts. A Sociological Explanation, Cambridge, Polity Press, 2012.

Johnston, Claire, « Women's Cinema as Counter-Cinema », in Johnston, Claire, Notes on Women's Cinema, Londres, Society for Education in Film and Television, 1973.

Kaufmann, Jean-Claude, La Femme seule et le Prince charmant, Paris, Nathan, 1999.

Kaufmann, Jean-Claude, L'Étrange histoire de l'amour heureux, Paris, Fayard, 2010.

Kaufmann, Jean-Claude, Corps de femmes, regards d'hommes. Sociologie des seins nus sur la plage, Paris, Pocket, 2010.

Kaufmann, Jean-Claude, « La force structurante d'une illusion : l'individu », in Corcuff, Philippe, Le Bart, Christian, Singly (de), François, L'Individu aujourd' hui. Débats sociologiques et contrepoints philosophiques, Rennes, Presses universitaires de Rennes, 2010.

Kaufmann, Jean-Claude,Sex@mour, Paris, Le Livre de Poche, 2011. Kinsey, Alfred, Sexual Behavior in the Human Male, Ishi Press, 2010. Kinsey, Alfred, Sexual Behavior in the Human Female, Ishi Press, 2010. Laclau, Ernesto, New Reflections on the Revolution of our Time, Londres, Verso, 1990. 
Laclau, Ernesto, Politics and Ideology in Marxist Theory. Capitalism, Fascism, Populism, Brooklyn, Verso, 2012.

Lauretis (de), Teresa, Alice Doesn't. Feminism, Semiotics, Cinema, Bloomington, Indiana University Press, 1984.

Lavery, David, Burkhead, Cynthia, Joss Whedon: Conversations, Jackson, University Press of Mississippi, 2011.

Lee, Janet, «Care to Join Me in an Upwardly Mobile Tango? Postmodernism and the "New Woman" », in Gammann, Lorraine, Marshment, Margaret, The Female Gaze. Women as Viewers of Popular Culture, St Paul, The Women's Press, 1988.

Leibman, Nina, Living Room Lectures: The Fifties Family in Film and Television, Austin, University of Texas Press, 1995.

Lotz, Amanda D., Redesigning Women. Television after the Network Era, Urbana, University of Illinois Press, 2006.

Lehman, Katherine, Those Girls. Single Women in Sixties and Seventies Popular Culture, Lawrence, University Press of Kansas, 2011.

Luhmann, Niklas, Amour comme passion. De la codification de l'intimité, Paris, Aubier Montaigne, 1992.

Macé, Éric, L'après-patriarcat, Paris, Seuil, 2015.

Macé, Éric, Les Imaginaires médiatiques. Une sociologie postcritique des médias, Paris, Amsterdam, 2006.

MacDonald, Myra, Representing Women. Myths of Femininity in the Popular Media, Londres, Hodder Education, 1995.

McCabe, Janet, Akass, Kim, Reading Desperate Housewives: Beyond the White Picket Fence, Londres, IB Tauris, 2006.

McLuhan, Marshall, Pour comprendre les médias, Paris, Seuil, 1977.

McRobbie, Angela, "Jackie": an Ideology of Adolescent Femininity, Birmingham, University of Birmingham, 1978.

McRobbie, Angela, The Aftermath of Feminism. Gender, Culture and Social Change, Londres, Sage Publications, 2009.

Maigret Éric, Macé, Éric, Penser les médiacultures. Nouvelles pratiques et nouvelles approches de la représentation du monde, Paris, Armand Colin, 2005.

Mellencamp, Patricia, «Situation Comedy, Feminism, And Freud: Discourses of Gracie and Lucy », in Butler, Jeremy, Star Texts: 
Image and Performance in Film and Television, Detroit, Wayne State University Press, 1991.

Modleski, Tania, Loving With A Vengeance. Mass-Produced Fantasies For Women, Londres, Routledge, 1984.

Modleski, Tania, Feminism Without Women. Culture And Criticism In A "Postfeminist" Age, Londres, Routledge, 1991.

Modleski, Tania, Hitchcock et la théorie féministe, Paris, L'Harmattan, 2002.

Morin, Céline, « L'amour et les théories de la communication », Questions de Communication, 26, 2014.

Morin, Céline, «Sexualités et redistribution des pouvoirs dans les séries américaines », Hermès, 69, 2014.

Morin, Céline, « Du romantisme à la relation pure ? Les amours des héroïnes de séries américaines depuis 1950 », Le Temps des Médias, 19, 2012.

Morin, Céline, « How To Love When You're a Good Wife », in Arp, Robert, Baltzer-Jaray, Kimberley, The Good Wife and Philosophy. Temptations of Saint Alicia, Chicago, Open Court Chicago, 2013.

Morin, Céline, « Les adolescentes et l'amour dans les séries télévisées, entre discours romantiques et pratiques passionnelles », in Julier-Costes, Martin, Jeffrey, Denis, Lachance, Jocelyn, Séries cultes et culte de la série chez les jeunes. Penser l'adolescence à travers les séries télévisées, Québec, Presses Universitaires de Laval, 2014.

Morin, Edgar, Commune en France, la métamorphose de Plozévet, Paris, Fayard, 1967.

Morin, Edgar, La Méthode, Paris, Seuil, 2008.

Morin, Edgar, L'Esprit du temps, Paris, Armand Colin, 2010.

Newcomb, Horace, Alley, Robert S., The Producer's Medium. Conversations with Creators of American TV, Oxford, Oxford University Press, 1985.

Ouellette, Laurie, « Inventing the Cosmo Girl. Class Identity and Girl-Style American Dreams », in Dines, Gall, Humez, Jean M., Gender, Race, and Class in Media: A Critical Reader, Londres, Sage Publications, 2011.

Pasquier, Dominique, Les scénaristes et la télévision. Approche sociologique, Paris, Nathan Université, 1999.

Penley, Constance, Feminist and Film Theory, Londres, Routledge, 1988. Péquignot, Bruno, La Relation amoureuse. Analyse sociologique du roman sentimental moderne, Paris, L'Harmattan, 1991. 
Press, Andrea L., Women Watching Television. Gender, Class, and Generation in the American Television Experience, Philadelphia, University of Pennsylvania Press, 1991.

Radway, Janice A., Reading the Romance. Women, Patriarchy and Popular Litterature, Chapel Hill, University of North Carolina Press, 1992.

Robinson, Lilliane, « What's My Line? Telefiction and Women's Work », in Sex, Class \& Culture, Bloomington, Indiana University Press, 1978.

Rosen, Ruth, The World Split Open: How the Modern Women's Movement Changed America, Londres, Penguin Books, 2006.

Rosenfelt, Deborah, Stacey, Judith, « Second Thoughts on the Second Wave », in Hansen, Karen, Philipson, Ilene, Women, Class and the Feminist Imagination. A Socialist Feminist Reader, Philadelphia, Temple University Press, 1990.

Rougemont (de), Denis, L'Amour et l'Occident, Paris, 10/18, 2001.

Rowe, Kathleen, The Unruly Woman: Gender and the Genres of Laughter in Understanding Inequality, Austin, University of Texas Press, 1995. Rubin, Gayle, « Thinking Sex: Notes for a Radical Theory of the Politics of Sexuality », in Vance, Carole S., Pleasure and Danger: Exploring Female Sexuality, Londres, Routledge, 1992.

Ryan, Mary P., Cradle of the Middle Class. The Family in Oneida County, New York, 1790-1865, Cambridge, Cambridge University Press, 1983. Shumway, David, Modern Love. Romance, Intimacy, And The Marriage Crisis, New York, New York University Press, 2003.

Singly (de), François, L'Individualisme est un humanisme, Paris, Éditions de l'Aube, 2007.

Singly (de), François, Séparée. Vivre l'expérience de la rupture, Paris, Armand Colin, 2011.

Spangler, Lynn C., Television Women from Lucy to Friends. Fifty Years of Sitcom and Feminism, Santa Barbara, Greenwood Press, 2003.

Spigel, Lynn, Make Room for TV. Television and the Family Ideal in Postwar America, Chicago, University of Chicago Press, 1992.

Spigel, Lynn, Mann, Denise, Private Screenings. Television and the Female Consumer, Minneapolis, University of Minnesota Press, 1992.

Spivak, Gayatri Chakravorty, Said, Edward, Guha, Ranajit, Selected Subaltern Studies, Oxford, Oxford University Press, 1988. 
Stacey, Jackie, Star Gazing: Hollywood Cinema and Female Spectatorship, Londres, Routledge, 1994.

Sullerot, Evelyne, La Presse féminine, Paris, Armand Colin, 1964.

Taylor, Ella, Prime-Time Families: Television Culture in Postwar America, Oakland, University of California Press, 1989.

Tucker, David C., The Women Who Made Television Funny: Ten Stars of 1950s Sitcoms, Jefferson, McFarland and Company, 2007.

Turk, Edward B., Marcel Carné et l'âge d'or du cinéma français, 1929-1945, Paris, L'Harmattan, 2002.

Van Zoonen, Liesbet, Feminist Media Studies, Londres, Sage Publications, 1994.

Wittig, Monique, La Pensée straight, Paris, Balland, 2001.

Žižek, Slavoj, « On virtual sex and related matters », in Gerbel, Karl, Weibel, Peter, Mythos Information. Welcome to the Wired World, Frankfurt, Springer Verlag, 1995.

\section{Articles de revue}

Amy-Chinn, Dee, « Queering the Bitch: Spike, Transgression and Erotic Empowerment », European Journal of Cultural Studies, 8, 3, 2005 , p. 313-328.

Andrieu, Bernard, « La fin de la biopolitique chez Michel Foucault : le troisième déplacement $\gg$, Le Portique, 13-14, 2004. Disponible en ligne : http://leportique.revues.org/627, consulté le 14 septembre 2014. Aronson, Pamela, «Feminists or "Postfeminists"?: Young Women's Attitudes toward Feminism and Gender Relations », Gender and Society, 17, 2003, p. 903-922.

Arthurs, Jane, «Sex and the City and Consumer Culture. Remediating Postfeminist Drama », Feminist Media Studies, 3, 1, 2003, p. 83-98.

Beck, Ulrich, Bonss, Wolfgang, Lau, Christoph, « The Theory of Reflexive. Modernization Problematic, Hypotheses and Research Programme », Theory, Culture \& Society, 20, 2, 2003, p. 1-33.

Bozon, Michel, Leridon, Henri, « Les constructions sociales de la sexualité », Populations, 5, 1993, p. 1173-1195.

Braidotti, Rosi, « Les sujets nomades féministes comme figure des multitudes $\gg$, Multitudes, 12, 2003, p. 27-38. 
Burch, Noël, «Double Speak. De l'ambiguïté tendancielle du cinéma hollywoodien », Réseaux, 99, 2000, p. 99-130.

Byars, Jackie, Meehan, Eileen, « Once in a Lifetime: Constructing “The Working Woman" through Cable Narrowcasting », Camera Obscura, 33-34, 1994-1995, p. 13-41.

Cooper, Brenda, « Unapologetic Women, “Comic Men” and Feminine Spectatorship in David E. Kelley's Ally McBeal », Critical Studies in Media Communication, 18, 4, 2001, p. 416-435.

Coulomb-Gully, Marlène, « Les sciences de l'information et de la communication : une discipline Gender blind ? », Questions de Communication, 15, 2009, p. 129-153.

Dow, Bonnie J., « Hegemony, Feminist Criticism and The Mary Tyler Moore Show », Critical Studies in Mass Communication, 7, 1990, p. 261-274.

Dow, Bonnie J., «Ally McBeal, Lifestyle Feminism, and the Politics of Personal Happiness », The Communication Review, 5, 4, 2002, p. 259-264.

Dubrosky, Rachel, « Ally McBeal as Postfeminist Icon: The Aestheticizing and Fetishizing of the Independent Working Woman », The Communication Review, 5, 4, 2002, p. 264-284.

Ehrenberg, Alain, « De la névrose à la dépression. Remarques sur quelques changements de l'individualité contemporaine », Figures de la psychanalyse, 4, 2001, p. 25-41.

Epstein, Michael, «Breaking the Celluloid Ceiling: Ally McBeal and the Women Attorneys Who Paved Their Way », Television Quaterly, 30, 1, 1999, p. 28-39.

Fraser, Nancy, « Repenser la sphère publique : contribution à la critique de la démocratie telle qu'elle existe réellement », Hermès, 31, 2001, p. 125-157.

Gerhard, Jane, « Sexand the City. Carrie Bradshaw's Queer Postfeminism », Feminist Media Studies, 5, 1, 2005, p. 37-49.

Gilligan, Carol, « Le care, éthique féminine ou éthique féministe ?», Multitudes, 37-38, 2009, p. 76-78.

Gitlin, Todd, « Prime Time Ideology. The Hegemonic Process in Television Entertainment », Social Problems, 26, 1979, p. 251-266. 
Grossberg, Lawrence, « History, Politics and Postmodernism: Stuart Hall and Cultural Studies », Journal of Communication Inquiry, 10, 1986, p. 61-77.

Grossberg, Lawrence, «On Postmodernism and Articulation: An Interview with Stuart Hall », Journal of Communication Inquiry, 10, 1986 , p. $45-107$.

Grossberg, Lawrence, « Does Cultural Studies Have Futures? Should It? (or What's the Matter with New York?) », Cultural Studies, 20, 1, 2006, p. 1-32.

Hall, Stuart, « The Problem of Ideology. Marxism without Guarantees », Journal of Communication Inquiry, 10, 28, 1986, p. 28-44.

Illouz, Eva, « Réseaux amoureux sur Internet », Réseaux, 138, 2006, p. 269-272.

Janus, Noreene Z., « Research on Sex-Roles in the Mass Media: Toward a Critical Approach », The Insurgent Sociologist, 7, 3, 1977, p. 19-31.

Kellner, Douglas, « Communication vs. Cultural Studies: Overcoming the Divide », Communication Theory, 5, 1995, p. 162-177.

Kim, L.S., « "Sex and the Single Girl" in Postfeminism: the F Word on Television », Television New Media, 2, 319, 2001, p. 319-334.

Laclau, Ernesto, « Ideology and postMarxism », Journal of Political Ideologies, 11, 2, 2006, p. 103-114.

Latour, Bruno, « Is Re-Modernization Occurring - And If So, How to Prove It? », Theory, Culture \& Society, 20, 2, 2003, p. 35-48.

Lentz, Kirsten Marthe, «Quality versus Relevance: Feminism, Race, and the Politics of the Sign in 1970s Television », Camera Obscura, 43, 2000, p. 44-93.

Lopate, Carol, «Daytime Television. You'll Never Want To Leave Home », Radical America, 11, 1977, p. 33-51.

Lotz, Amanda, « Postfeminist Television Criticism: Rehabilitating Critical Terms and Identifying Postfeminist Attributes $\gg$, Feminist Media Studies, 1, 2001, p. 105-121.

Macé, Éric, «Qu'est-ce qu'une sociologie de la télévision ? Esquisse d'une théorie des rapports sociaux médiatisés », Réseaux, 104, 2001, p. 245-288.

Macé, Éric, « Le piège de la “cause des femmes”. Éléments pour un mouvement antisexiste postféministe », Cosmopolitiques, 4, 2003, p. 84-103. 
Macé, Éric, « Le conformisme provisoire de la programmation », Hermès, 37, 2003, p. 127-135.

Maigret, Éric, «La Maison mentale du masculin: Dexter, House, Mentalist », MédiaMorphoses, 26, 2010, p. 74-78.

Markle, Gail, « "Can Women Have Sex Like a Man? Sexual Scripts in Sex and the City », Sexuality \& Culture, 12, 1, 2008, p. 45-57.

Mattelart, Michèle, « Femmes et médias. Retour sur une problématique », Réseaux, 4, 120, 2003, p. 23-51.

McCabe, Janet, Akass, Kim, « Feminist Television Criticism: Notes and Queries », Critical Studies in Television, 1, 2006, p. 108-120.

McKenna, Susan E., «The Queer Insistance of Ally McBeal: Lesbian Chic, Postfeminism, and Lesbian Reception », The Communication Review, 5, 4, 2002, p. 285-314.

Mercier, Arnaud, « Quand le bouffon franchit le Rubicon : la candidature Coluche à la présidentielle de $1981 »$, Hermès, 29, 2001, p. 175-183.

Molinier, Pascale, «Formaliser et politiser les récits du care », Interrogations, 11, 2010. Disponible en ligne : http://www.revue-interrogations.org/fichiers/223/Varia, consulté le 14 septembre 2014.

Molinier, Pascale, «Au-delà de la féminité et du maternel, le travail du care », L'Esprit du temps, 58, 2010, p. 161-174.

Moseley, Rachel, Read, Jacinda, « “Having It Ally”: Popular Television (Post)Feminism », Feminist Media Studies, 2, 2002, p. 231-249.

Moseley, Rachel, «Glamorous Witchcraft. Gender and Magic in Teen Film and Television », Screen, 43, 2002, p. 403-422.

Mulvey, Laura, «Visual Pleasure and Narrative Cinema », Screen, 16, 3 , 1975, p. 6-18.

Mulvey, Laura, « Afterthoughts on "Visual Pleasure and Narrative Cinema" inspired by King Vidor's Duel in the Sun (1946) », Framework, 15-1617, 1981, p. 12-15.

Ouellette, Laurie, « Victims No More: Postfeminism, Television, and Ally McBeal », The Communication Review, 5, 4, 2002, p. 315-335.

Owens Patton, Tracy, «Ally McBeal and her Homies. The Reification of White Stereotypes of the Other », Journal of Black Studies, 32, 2, 2001, p. 229-260.

Péquignot, Bruno, «Les femmes dans le roman sentimental moderne », L'Homme et la société, 99-100, 1991, p. 115-125. 
Probyn, Elspeth, « New Traditionalism and PostFeminism: TV Does the Home », Screen, 31, 2, 1990, p. 147-159.

Richardson, Niall, « As Kamp as Bree. The Politics of Camp Reconsidered by Desperate Housewives », Feminist Media Studies, 6, 2006, p. 157-174.

Rollet, Brigitte, Sellier, Geneviève, «Cinéma et genre en France : état des lieux », Clio, 1999, 10. Disponible en ligne : http://clio.revues. org/1533, consulté le 14 septembre 2014.

Rowe, Kathleen, « Roseanne: Unruly Woman as Domestic Goddess », Screen, 31, 1990, p. 408-410.

Sellier, Geneviève, «Images de femmes dans le cinéma de la Nouvelle Vague », Clio, 10, 2, 1992. Disponible en ligne : http://clio.revues. org/265?lang=fr, consulté le 14 septembre 2014.

Sellier, Geneviève, « Gender studies et études filmiques », Cabiers du genre, 38, 1, 2005, p. 63-85.

Singly (de), François, «Intimité conjugale et intimité personnelle : à la recherche d'un équilibre entre deux exigences dans les sociétés modernes avancées $\gg$, Sociologie et sociétés, 35, 2, 2003, p. 79-96.

Steeves, Leslie H., « Feminist Theories and Media Studies », Critical Studies in Mass Communication, 4, 2, 1987, p. 95-135.

Tuchman, Gaye, « Women's Depiction in the Mass Media », Signs, 3, 1979 , p. 528-542.

\section{Ressources audiovisuelles et articles de presse}

«United States of Television. America in Prime Time. Épisode 1 : The Independent Woman », PBS, 2011.

« The Good Wife: Non-Lawyers Behind That Lawyer Show », Bitter Lawyer, 4 janvier 2010. Disponible en ligne : http://www.bitterlawyer. com/the-good-wife-non-lawyers-behind-that-lawyer-show/, consulté le 22 septembre 2014.

« Donna Reed, oscar winner and TV star, is dead at 64 », New York Times, 15 juillet 1986, référence faite à une interview du New York Times de 1964. 
«Quayle says television contributed to the L.A. riots, says "Murphy Brown" mocked the importance of marriage », Los Angeles Times, 20 mai 1992.

Barr, Roseanne, « And I Should Know », New York Magazine, 15 mai 2011.

Bragman, Bob, «I Love Ethel. A Vivian Vance archive uncovered », San Francisco Chronicle, 19 janvier 2010.

Bellafante, Ginia, «Feminist: It's All About Me! Want to know what today's chic young feminist thinkers care about? Their bodies! Themselves! », Time, 29 juin 1008.

Bremner, Jade, « Edgy comedy about dealing with cancer comes to screens in Dubai », Time Out Dubai, 22 mars 2011.

Callaghan, Dylan, «Jackie, Blue », Writer's Guild of America. Disponible en ligne : http://www.wga.org/content/default.aspx?id=3674, consulté le 22 septembre 2014.

Carter, Bill, «With "Homeland", Showtime Makes Gains on HBO », New York Times, 29 janvier 2012. Disponible en ligne : http://www. nytimes.com/2012/01/30/business/media/with-homeland-showtime-makes-gains-on-hbo.html? pagewanted=all\&_r $=0$, consulté le 22 septembre 2014.

Douglas, Susan, « Where have you gone, Roseanne Barr? », The Shriver Report, 9 novembre 2009.

Fallon, Kevin, « Showtime: Television for Women, for Everyone? », The Atlantic, 16 août 2010, disponible en ligne : http://www.theatlantic.com/entertainment/archive/2010/08/showtime-television-for-women-for-everyone/61535/, consulté le 22 septembre 2014.

Goldberg, Lesley, «"The Big C" Postmortem: Cathy Was "Lucky and Unlucky at the Same Time" », Hollywood Reporter, 20 mai 2013.

Halterman, Jim, «Interview: "Nurse Jackie" Co-Creator Liz Brixius », The Futon Critic, 12 avril 2010. Disponible en ligne : http://www. thefutoncritic.com/interviews/2010/04/12/interview-nurse-jackieco-creator-liz-brixius-35266/20100412_nursejackie/, consulté le 22 septembre 2014.

Jacobs, A.J., «Interview with a Vampire Chronicler », Entertainment Weekly, 25 avril 1997. Disponible en ligne : http://www.ew.com/ew/ article/0,,287570,00.html, consulté le 14 septembre 2014. 
Kinon, Cristina, « "Nurse Jackie" Star Edie Falco, Mary-Louise Parker, more strong women lift Showtime », Daily News, 15 février 2009, disponible en ligne : http://www.nydailynews.com/entertainment/ tv-movies/nurse-jackie-star-edie-falco-mary-louise-parker-strong-women-lift-show time-article-1.363757, consulté le 22 septembre 2014.

Pierce, Scott D., «TV: “Desperate Housewives" creator owes it all to mom », The Salt Lake Tribune, 10 mai 2012.

Shales, Tom, « Stereotypes in Videoland », Washington Post, 17 août 1977. 